\title{
Three-dimensional Fracture Mechanics Analyses of Single Edge Cracked Plate Specimens
}

\author{
by \\ ZHAOYU JIN, M. Eng. \\ A thesis submitted to the Faculty of Graduate and Postdoctoral \\ Affairs in partial fulfillment of the requirements \\ for the degree of
}

Master of Applied Science

In

Mechanical Engineering

Carleton University

Ottawa, Ontario

(C) 2011

ZHAOYU JIN 
Library and Archives

Canada

Published Heritage

Branch

395 Wellington Street

Ottawa ON K1A ON4

Canada
Bibliothèque et

Archives Canada

Direction du

Patrimoine de l'édition

395 , rue Wellington

Ottawa ON K1A ON4

Canada
Your file Votre référence

ISBN: 978-0-494-87797-5

Our file Notre référence

ISBN: 978-0-494-87797-5

\section{NOTICE:}

The author has granted a nonexclusive license allowing Library and Archives Canada to reproduce, publish, archive, preserve, conserve, communicate to the public by telecommunication or on the Internet, loan, distrbute and sell theses worldwide, for commercial or noncommercial purposes, in microform, paper, electronic and/or any other formats.

The author retains copyright ownership and moral rights in this thesis. Neither the thesis nor substantial extracts from it may be printed or otherwise reproduced without the author's permission.
AVIS:

L'auteur a accordé une licence non exclusive permettant à la Bibliothèque et Archives Canada de reproduire, publier, archiver, sauvegarder, conserver, transmettre au public par télécommunication ou par l'Internet, prêter, distribuer et vendre des thèses partout dans le monde, à des fins commerciales ou autres, sur support microforme, papier, électronique et/ou autres formats.

L'auteur conserve la propriété du droit d'auteur et des droits moraux qui protege cette thèse. $\mathrm{Ni}$ la thèse ni des extraits substantiels de celle-ci ne doivent être imprimés ou autrement reproduits sans son autorisation.
In compliance with the Canadian Privacy Act some supporting forms may have been removed from this thesis.

While these forms may be included in the document page count, their removal does not represent any loss of content from the thesis.
Conformément à la loi canadienne sur la protection de la vie privée, quelques formulaires secondaires ont été enlevés de cette thèse.

Bien que ces formulaires aient inclus dans la pagination, il n'y aura aucun contenu manquant. 
The undersigned recommend to

The Faculty of Graduate Studies and Research acceptance of the thesis

\title{
Three-dimensional Fracture Mechanics Analyses of Single Edge Cracked Plate Specimens
}

\author{
submitted by \\ ZHAOYU JIN, M. Eng. \\ In partial fulfillment of the requirements for \\ The degree of Master of Applied Science (Mechanical)
}

$X$. Wang, Thesis supervisor

Metin I. Yaras, Chair, Department of Mechanical and Aerospace Engineering

Carleton University

December, 2011 


\section{ABSTRACT}

In this thesis, the stress intensity factor $K_{I}, T_{11}$ and $T_{33}$ stresses along the crack front of the single edge cracked plate (SECP) specimens with the crack surface subjected to uniform, linear, parabolic or cubic stress distribution are calculated by three-dimensional finite element analyses. The relative crack depth, $a / W$, is varied by $0.2,0.4,0.6$ or 0.8 . And the relative thickness, $t / W$, is chosen by $0.1,0.2,0.5,1.0,2.0$ or 4.0, respectively. For engineering applications, empirical equations of normalized stress intensity factor $K_{\mathrm{I}}, T_{11}$ and $T_{33}$ stress at the mid-plane are obtained.

The thickness effect for the three parameters of stress intensity factor $K_{l}, T_{11}$ and $T_{33}$ stresses at the mid-plane of the SECP specimen is discussed in this thesis. The stress distribution applied on the crack surface is uniform pressure. In order to study the thickness effect completely, additional cases with relative thickness, $t / W=0.0025,0.025$, $0.05,8,16$ for crack surface subjected to the uniform stress distribution are calculated by finite element analyses. In addition, the relative crack depth effect of the stress intensity factor $K_{l}, T_{11}$ and $T_{33}$ stresses at the mid-plane of the SECP specimen is discussed as well. The three parameters variation along the crack front are also investigated.

The stress state around the crack front in the three-dimensional SECP specimens described by the stress intensity factor $K_{I}, T_{11}$ and $T_{33}$ stresses are compared to the results from the finite element analyses. The differences between the Williams' expansion and finite element analyses varied by angle, radius and thickness are presented. 


\section{ACKNOWLEDGEMENTS}

I am heartily thankful to my supervisor, Professor Xin Wang, whose encouragement, guidance and support from the initial to the final stage of the research enabled me to develop an understanding of the subject. I would like to express my love and gratitude to my wife, Weiwei Zhong and my son, Zhongqi Jin; for their understanding and endless love, through the duration of my studies.

Financial support from the Natural Sciences and Engineering Research Council

of Canada (NSERC) in the form of research grants to Professor Xin Wang is gratefully acknowledged. 


\section{CONTENTS}

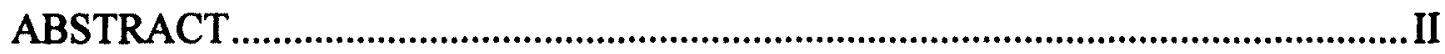

ACKNOWLEDGEMENTS ............................................................................II

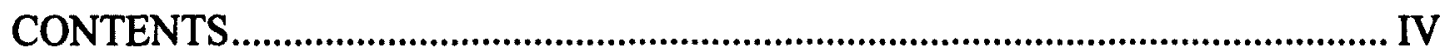

LIST of TABLES …….....................................................................................

LIST of FIGURES

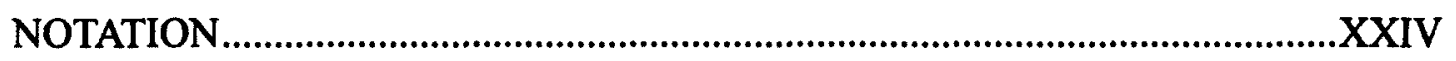

CHAPTER 1

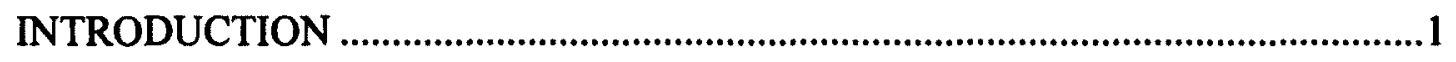

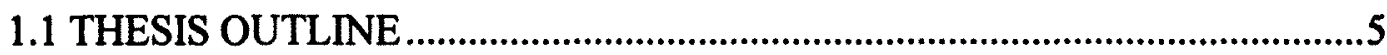

CHAPTER 2

BACKGROUND AND LITERATURE REVIEW ..................................................6

2.1 LINEAR ELASTIC FRACTURE MECHANICS ............................................6

2.1.1 Fracture Modes and Stress Intensity Factor............................................6

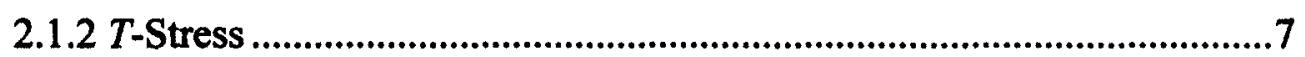

2.1.3 Superposition Method for the determination of $K, T_{11}$ AND $T_{33} \ldots \ldots \ldots . . .9$

2.2 FINITE ELEMENT ANALYSIS OF CRACKED COMPONETS ...................11

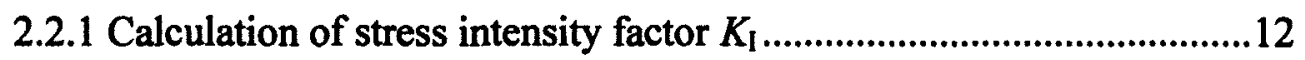

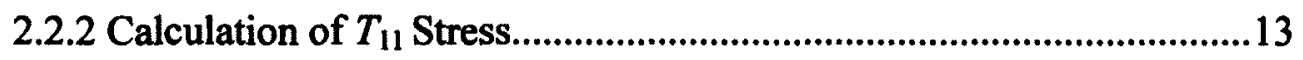

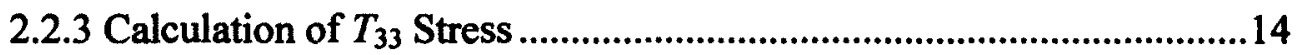

2.3 LITERATURE REVIEW FOR THREE-DIMENSIONAL SOLUTION OF $K$, $T_{11}$ AND $T_{33}$ AND STRESS STATE AROUND CRACK FRONT .................15

2.4 SINGLE EDGE CRACKED PLATE SPECIMEN ...........................................19

2.4.1 Two dimensional solutions for single edge cracked plate specimen ...20

2.4.2 Three dimensional solutions for single edge cracked plate specimen and the thickness effect.................................................................................20 
2.4.3 The present research for single edge cracked plate

.23

\section{CHAPTER 3}

STRESS INTENSITY FACTOR AND T-STRESSES FOR CRACK IN THREE DIMENSIONAL SINGLE EDGE CRACKED PLATE SPECIMENS ........................28

3.1 FINITE ELEMENT ANALYSIS FOR CRACK IN TRHEE-DIMENSIONAL SINGLE EDGE CRACKED PLATE SPECIMENS ........................................28

3.1.1 Finite Element Model ......................................................................28

3.1.2 Verification of the Finite Element Model .............................................30

3.1.3 Finite Element Results.........................................................................31

3.2 EMPIRICAL EQUATIONS OF STRESS INTENSITY FACTOR AND

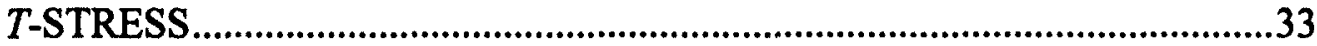

3.2.1 Stress Intensity Factor Equations..........................................................33

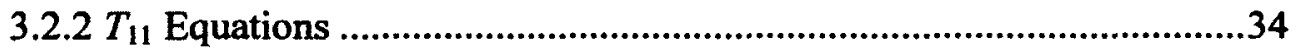

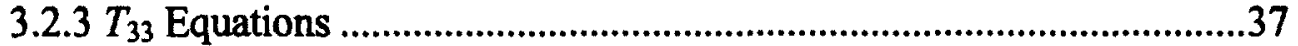

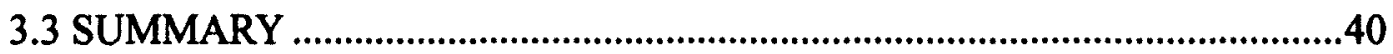

CHAPTER 4

VARIATIONS OF $K_{\mathrm{I}}, T_{11}$ AND $T_{33}$ BY GEOMETRY ...........................................90

4.1 Mid-plane $K_{\mathrm{I}}, T_{11}$ and $T_{33}$ Varied by Geometry ...............................................90

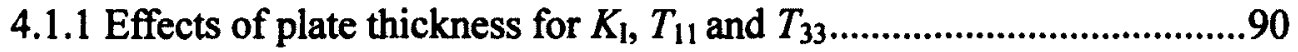

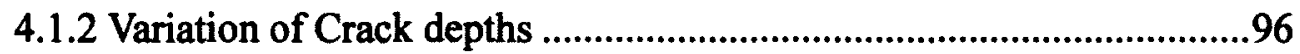

$4.2 K_{1}, T_{11}$ and $T_{33}$ Variation along the Crack Front ...........................................97

CHAPTER 5

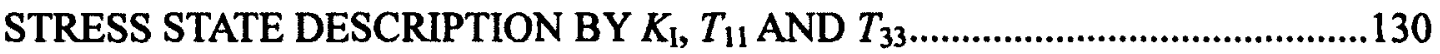

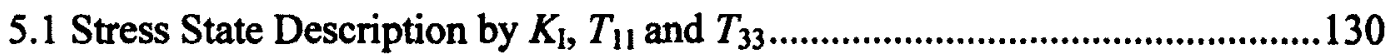

5.1.1 Stress State Varied by Angle ....................................................................131

5.1.2 Stress State Varied by Radius ........................................................132

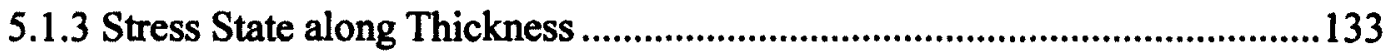


5.2 Conclusion

CHAPTER 6

CONCLUSIONS AND RECOMMENDATIONS ....................................................176

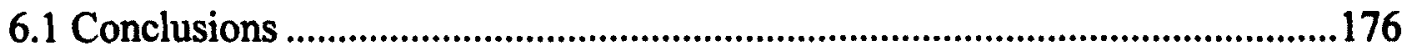

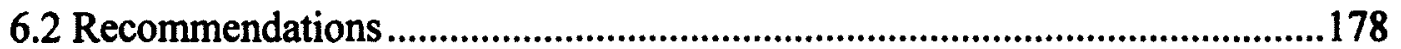

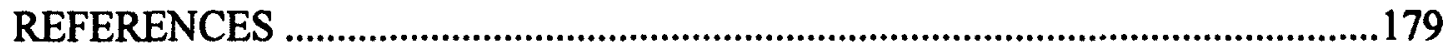




\section{LIST of TABLES}

Table 3.1 Comparison of the K from present FEM calculation and solution form Qu and

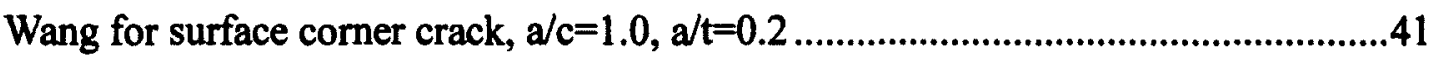

Table 3.2 Comparison of the $T_{11}$ from present FEM calculation and solution form $\mathrm{Qu}$ and Wang for surface corner crack, $a / c=1.0, a / t=0.2$

Table 3.3 Comparison of the $T_{33}$ from present FEM calculation and solution form $\mathrm{Qu}$ and Wang for surface corner crack, $a / c=1.0, a / t=0.2$

Table 3.4 Comparison of $T_{11}$ from present FEM calculation and solution from Nakamura and Parks (1992) for $\mathrm{a} / \mathrm{W}=0.5, \mathrm{t} / \mathrm{W}=0.5$

Table 3.5 Comparison of stress intensity factor and $T_{11}$ from present FEM calculation and solution from Sham (1991) for $a / W=0.5$

Table 3.6 Normalized $K_{1}, T_{11}$ and $T_{33}$ for $\mathrm{a} / \mathrm{W}=0.2, \mathrm{t} / \mathrm{W}=0.1, \mathrm{SECP}$

Table 3.7 Normalized $K_{1}, T_{11}$ and $T_{33}$ for $\mathrm{a} / \mathrm{W}=0.2, \mathrm{t} / \mathrm{W}=0.2$, SECP

Table 3.8 Normalized $K_{\mathrm{I}}, T_{11}$ and $T_{33}$ for $\mathrm{a} / \mathrm{W}=0.2, \mathrm{t} / \mathrm{W}=0.5$, SECP

Table 3.9 Normalized $K_{1}, T_{11}$ and $T_{33}$ for $\mathrm{a} / \mathrm{W}=0.2, \mathrm{t} / \mathrm{W}=1.0, \mathrm{SECP}$.

Table 3.10 Normalized $K_{\mathrm{f}}, T_{11}$ and $T_{33}$ for $\mathrm{a} / \mathrm{W}=0.2, \mathrm{t} / \mathrm{W}=2.0, \mathrm{SECP}$

Table 3.11 Normalized $K_{\mathrm{l}}, T_{11}$ and $T_{33}$ for $\mathrm{a} / \mathrm{W}=0.2, \mathrm{t} / \mathrm{W}=4.0, \mathrm{SECP}$

Table 3.12 Normalized $K_{1}, T_{11}$ and $T_{33}$ for $\mathrm{a} / \mathrm{W}=0.4, \mathrm{t} / \mathrm{W}=0.1, \mathrm{SECP}$ .50 
Table 3.13 Normalized $K_{1}, T_{11}$ and $T_{33}$ for $\mathrm{a} / \mathrm{W}=0.4, \mathrm{t} / \mathrm{W}=0.2$, SECP.

Table 3.14 Normalized $K_{1}, T_{11}$ and $T_{33}$ for $\mathrm{a} / \mathrm{W}=0.4, \mathrm{t} / \mathrm{W}=0.5$, SECP. .52

Table 3. 15 Normalized $K_{\mathrm{I}}, T_{11}$ and $T_{33}$ for $\mathrm{a} / \mathrm{W}=0.4, \mathrm{t} / \mathrm{W}=1.0, \mathrm{SECP}$ .53

Table 3.16 Normalized $K_{\mathrm{I}}, T_{11}$ and $T_{33}$ for $\mathrm{a} / \mathrm{W}=0.4, \mathrm{t} / \mathrm{W}=2.0, \mathrm{SECP}$. 54

Table 3.17 Normalized $K_{\mathrm{I}}, T_{11}$ and $T_{33}$ for $\mathrm{a} / \mathrm{W}=0.4, \mathrm{t} / \mathrm{W}=4.0$, SECP. .55

Table 3.18 Normalized $K_{1}, T_{11}$ and $T_{33}$ for $\mathrm{a} / \mathrm{W}=0.6, \mathrm{t} / \mathrm{W}=0.1, \mathrm{SECP}$. .56

Table 3.19 Normalized $K_{1}, T_{11}$ and $T_{33}$ for $\mathrm{a} / \mathrm{W}=0.6, \mathrm{t} / \mathrm{W}=0.2$, SECP. 57

Table 3.20 Normalized $K_{\mathrm{I}}, T_{11}$ and $T_{33}$ for $\mathrm{a} / \mathrm{W}=0.6, \mathrm{t} / \mathrm{W}=0.5$, SECP. .58

Table 3.21 Normalized $K_{1}, T_{11}$ and $T_{33}$ for $\mathrm{a} / \mathrm{W}=0.6, \mathrm{t} / \mathrm{W}=1.0, \mathrm{SECP}$

Table 3.22 Normalized $K_{\mathrm{l}}, T_{11}$ and $T_{33}$ for $\mathrm{a} / \mathrm{W}=0.6, \mathrm{t} / \mathrm{W}=2.0, \mathrm{SECP}$. .60

Table 3.23 Normalized $K_{\mathrm{I}}, T_{11}$ and $T_{33}$ for $\mathrm{a} / \mathrm{W}=0.6, \mathrm{t} / \mathrm{W}=4.0, \mathrm{SECP}$. 61

Table 3.24 Normalized $K_{1}, T_{11}$ and $T_{33}$ for $\mathrm{a} / \mathrm{W}=0.8, \mathrm{t} / \mathrm{W}=0.1, \mathrm{SECP}$. .62

Table 3.25 Normalized $K_{1}, T_{11}$ and $T_{33}$ for $\mathrm{a} / \mathrm{W}=0.8, \mathrm{t} / \mathrm{W}=0.2, \mathrm{SECP}$. .63

Table 3.26 Normalized $K_{\mathrm{l}}, T_{11}$ and $T_{33}$ for $\mathrm{a} / \mathrm{W}=0.8, \mathrm{t} / \mathrm{W}=0.5$, SECP. .64

Table 3.27 Normalized $K_{1}, T_{11}$ and $T_{33}$ for $\mathrm{a} / \mathrm{W}=0.8, \mathrm{t} / \mathrm{W}=1.0, \mathrm{SECP}$. 65

Table 3.28 Normalized $K_{\mathrm{l}}, T_{11}$ and $T_{33}$ for $\mathrm{a} / \mathrm{W}=0.8, \mathrm{t} / \mathrm{W}=2.0, \mathrm{SECP}$. .66

Table 3.29 Normalized $K_{\mathrm{l}}, T_{11}$ and $T_{33}$ for $\mathrm{a} / \mathrm{W}=0.8, \mathrm{t} / \mathrm{W}=4.0, \mathrm{SECP}$ . .67 
Table 3.30 Parameters of the empirical formulae for stress intensity factor $K_{1}$

Table 4.1 Finite element result for $a / W=0.2$, subjected to uniform load, SECP. .99

Table 4.2 Finite element result for $a / W=0.4$, subjected to uniform load, SECP .99

Table 4.3 Finite element result for $a / W=0.6$, subjected to uniform load, SECP .99

Table 4.4 Finite element result for $a / W=0.8$, subjected to uniform load, SECP 100

Table 4. 5 2D solution, crack subjected to uniform load, SECP, Sham (1991) 100

Table 4.6 Values of $B^{2 D}$ and $B^{\text {thin }}(0)$ for $a / W=0.2,0.4,0.6$ and 0.8 subjected to uniform load on the crack surface 100

Table 4.7 The comparison of the approximate thickness-dependence of $T_{11}$ stress at mid-plane as given by Eq. (4.5) and the finite element results, Uniform, SECP, $a / W=0.2$ .101

Table 4.8 The comparison of the approximate thickness-dependence of $T_{11}$ stress at mid-plane as given by Eq. (4.5) and the finite element results, Uniform, SECP, $a / W=0.4$ .101

Table 4.9 The comparison of the approximate thickness-dependence of $T_{11}$ stress at mid-plane as given by Eq. (4.5) and the finite element results, Uniform, SECP, $a / W=0.6$

Table 4.10 The comparison of the approximate thickness-dependence of $T_{11}$ stress at mid-plane as given by Eq. (4.5) and the finite element results, Uniform, SECP, $a / W=0.8$ 102 
Table 4.11 Values of $B_{33}{ }^{2 D}$ and $B_{33}{ }^{\text {thin }}(0)$ for $a / W=0.2,0.4,0.6$ and 0.8 subjected to uniform load on the crack surface.

Table 4. 12 The comparison of the approximate thickness-dependence of $T_{33}$ stress at mid-plane as given by Eq. (4.10) and the finite element results, Uniform, SECP, $a / W=0.2$.

Table 4.13 The comparison of the approximate thickness-dependence of $T_{33}$ stress at mid-plane as given by Eq. (4.10) and the finite element results, Uniform, SECP, $a / W=0.4$ 104

Table 4.14 The comparison of the approximate thickness-dependence of $T_{33}$ stress at mid-plane as given by Eq. (4.10) and the finite element results, Uniform, SECP, $a / W=0.6$.

Table 4.15 The comparison of the approximate thickness-dependence of $T_{33}$ stress at mid-plane as given by Eq. (4.10) and the finite element results, Uniform, SECP, $a / W=0.8$ 105

Table 4.16 The thickness correction factor $\Delta K_{3 \mathrm{D}}$ for $a / W=0.2$ 105

Table 4.17 The thickness correction factor $\Delta K_{3 \mathrm{D}}$ for $a / W=0.4$ .106

Table 4.18 The thickness correction factor $\Delta K_{3 \mathrm{D}}$ for $a / W=0.6$ 106

Table 4.19 The thickness correction factor $\Delta K_{3 \mathrm{D}}$ for $a / W=0.8$ 107

Table 4.20 The thickness correction factor $\Delta T_{113 \mathrm{D}}$ for $a / W=0.2$ .107

Table 4.21 The thickness correction factor $\Delta T_{113 \mathrm{D}}$ for $a / W=0.4$ 108 
Table 4.22 The thickness correction factor $\Delta T_{113 \mathrm{D}}$ for $a / W=0.6$

Table 4. 23 The thickness correction factor $\Delta T_{113 \mathrm{D}}$ for $a / W=0.8$ 109

Table 4.24 The thickness correction factor $\Delta T_{333 \mathrm{D}}$ for $a / W=0.2$ 109

Table 4.25 The thickness correction factor $\Delta T_{333 \mathrm{D}}$ for $a / W=0.4$

Table 4.26 The thickness correction factor $\Delta T_{333 \mathrm{D}}$ for $a / W=0.6$ 110

Table 4.27 The thickness correction factor $\Delta T_{333 \mathrm{D}}$ for $a / W=0.8$

Table 5.1 Comparison of stress state at mid-plane between finite element results and formulae evaluation for $a / W=0.8, t / W=0.1$, Uniform Load, SECP

Table5.2 Comparison of stress state at mid-plane between finite element results and formulae evaluation for $a / W=0.8, t / W=0.2$, Uniform Load, SECP 136

Table 5.3 Comparison of stress state at mid-plane between finite element results and formulae evaluation for $a / W=0.8, t / W=0.5$, Uniform Load, SECP.

Table 5.4 Comparison of stress state at mid-plane between finite element results and formulae evaluation for $a / W=0.8, t / W=1.0$, Uniform Load, SECP.

Table 5.5 Comparison of stress state at mid-plane between finite element results and formulae evaluation for $a / W=0.8, t / W=2.0$, Uniform Load, SECP

Table 5.6 Comparison of stress state at mid-plane between finite element results and formulae evaluation for $a / W=0.8, t / W=4.0$, Uniform Load, SECP 
Table 5.7 Percent different between Williams formula and FEM, $a / W=0.6, t / W=0.5$, Uniform Load, SECP

Table 5.8 Stress State along the circular path close to the front varied along thickness, $a / W=0.6, t / W=0.1, r / a=0.0078$, Uniform, SECP 142

Table 5.9 Stress State along the circular path close to the front varied along thickness, $a / W=0.6, t / W=0.2, r / a=0.0078$, Uniform, SECP 143

Table 5.10 Stress State along the circular path close to the front varied along thickness, $a / W=0.6, t / W=0.5, r / a=0.0078$, Uniform, SECP 144

Table 5.11 Stress State along the circular path close to the front varied along thickness, $a / W=0.6, t / W=1.0, r / a=0.0078$, Uniform, SECP 145

Table 5.12 Stress State along the circular path close to the front varied along thickness, $a / W=0.6, t / W=2.0, r / a=0.0078$, Uniform, SECP .146

Table 5.13 Stress State along the circular path close to the front varied along thickness, $a / W=0.6, t / W=4.0, r / a=0.0078$, Uniform, SECP .147 


\section{LIST of FIGURES}

Figure 2.1 The three independent modes of movement of crack surfaces.

Figure 2.2 Three-dimensional coordinate system for the region along the crack front... .24

Figure 2.3 Principle of superposition, two-dimensional .25

Figure 2.4 Principle of superposition, three-dimensional .26

Figure 2.5 A schematic of elements used in the definition of the interaction integral: (a), (b), (c) .27

Figure 2.6 Single edge cracked specimen

Figure 3.1 Geometric parameters of single edge cracked plate specimen with crack surface subjected to stress distribution $\sigma(x)$.

Figure 3.2 C3D20 element and mesh configuration near the crack tip .70

Figure 3.3 Finite element model for $a / W=0.4, t / W=0.5$, SECP .71

Figure 3.4 Corner crack in the finite thickness plate .72

Figure 3.5 Surface corner crack model in ABAQUS, $a / c=1.0, a / t=0.2$

Figure 3.6 Comparison of the $T_{11}$ from present FEM calculation and solution form $\mathrm{Qu}$ and Wang (2006) for surface corner crack, $a / c=1.0, a / t=0.2$ 
Figure 3.7 Comparison of the $T_{33}$ from present FEM calculation and solution form $\mathrm{Qu}$ and Wang (2006) for surface corner crack, $a / c=1.0, a / t=0.2$

Figure 3.8 Variation of normalized $T_{11}$ along front of an edge-cracked plate for various Poisson's ratios under tension .74

Figure 3.9 Normalized Stress Intensity Factor, $a / W=0.2$, Uniform, SECP . .75

Figure 3.10 Normalized Stress Intensity Factor, $a / W=0.2$, Linear, SECP . .75

Figure 3.11 Normalized $T_{11}, a / W=0.2$, Uniform, SECP . .76

Figure 3.12 Normalized $T_{11}, a / W=0.2$, Linear, SECP . .76

Figure 3.13 Normalized $T_{33}, a / W=0.2$, Uniform, SECP .77

Figure 3.14 Normalized $T_{33}, a / W=0.2$, Linear, SECP . .77

Figure 3.15 Normalized Stress Intensity Factor, $a / W=0.4$, Uniform, SECP . .78

Figure 3.16 Normalized Stress Intensity Factor, $a / W=0.4$, Linear, SECP . .78

Figure 3.17 Normalized $T_{11}, a / W=0.4$, Uniform, SECP . .79

Figure 3.18 Normalized $T_{11}, a W=0.4$, Linear, SECP .79

Figure 3.19 Normalized $T_{33}, a / W=0.4$, Uniform, SECP . .80

Figure 3.20 Normalized $T_{33}, a / W=0.4$, Linear, SECP . .80

Figure 3.21 Normalized Stress Intensity Factor, $a / W=0.6$, Uniform, SECP . .81 
Figure 3.22 Normalized Stress Intensity Factor, $a / W=0.6$, Linear, SECP

Figure 3.23 Normalized $T_{11}, a / W=0.6$, Uniform, SECP .82

Figure 3. 24 Normalized $T_{11}, a / W=0.6$, Linear, SECP .82

Figure 3.25 Normalized $T_{33}, a / W=0.6$, Uniform, SECP . .83

Figure 3.26 Normalized $T_{33}, a / W=0.6$, Linear, SECP. .83

Figure 3.27 Normalized Stress Intensity Factor, $a / W=0.8$, Uniform, SECP .84

Figure 3.28 Normalized Stress Intensity Factor, $a / W=0.8$, Linear, SECP .84

Figure 3.29 Normalized $T_{11}, a / W=0.8$, Uniform, SECP .85

Figure 3.30 Normalized $T_{11}, a / W=0.8$, Linear, SECP. .85

Figure 3.31 Normalized $T_{33}, a / W=0.8$, Uniform, SECP .86

Figure 3.32 Normalized $T_{33}, a / W=0.8$, Linear, SECP. .86

Figure 3.33 Comparison of numerical results and empirical formulae for stress intensity factor: (a) $t / W=0.1$, (b) $t / W=0.2$, (c) $t / W=0.5$, (d) $t / W=1.0$, (e) $t / W=2.0$, and (f) $t / W=4.0$

Figure 3.34 Comparison of numerical results and empirical formulae for $T_{11}$ : (a) $t / W=0.1$, (b) $t / W=0.2$, (c) $t / W=0.5$, (d) $t / W=1.0$, (e) $t / W=2.0$, and (f) $t / W=4.0$ .88

Figure 3.35 Comparison of numerical results and empirical formulae for $T_{33}$ : (a) $t / W=0.1$, (b) $t / W=0.2$, (c) $t / W=0.5$, (d) $t / W=1.0$, (e) $t / W=2.0$, and (f) $t / W=4.0$ .89 
Figure 4.1 Mid-plane value of $K_{\mathrm{I}}$ as a function of plate thickness in SECP,

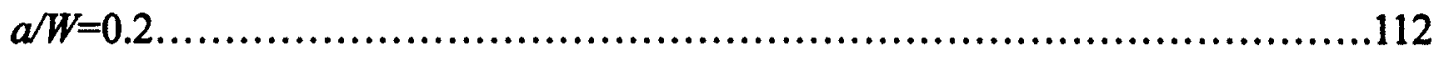

Figure 4.2 Mid-plane value of $K_{\mathrm{I}}$ as a function of plate thickness in SECP, $a / W=0.4 \ldots .112$

Figure 4.3 Mid-plane value of $K_{\mathrm{I}}$ as a function of plate thickness in SECP, $a / W=0.6 \ldots .113$

Figure 4.4 Mid-plane value of $K_{1}$ as a function of plate thickness in SECP, $a / W=0.8 \ldots .113$

Figure 4.5 Mid-plane value of $T_{11}$ stress as a function of plate thickness in SECP, $a / W=0.2$.

Figure 4.6 Mid-plane value of $T_{11}$ stress as a function of plate thickness in SECP, $a / W=0.4$

Figure 4.7 Mid-plane value of $T_{11}$ stress as a function of plate thickness in SECP, $a / W=0.6$.

Figure 4.8 Mid-plane value of $T_{11}$ stress as a function of plate thickness in SECP, $a / W=0.8$

Figure 4.9 Comparison of approximation formula and FEM for $T_{11}, a / W=0.2$

Figure 4.10 Comparison of approximation formula and FEM for $T_{11}, a / W=0.4$ 116

Figure 4.11 Comparison of approximation formula and FEM for $T_{11}, a / W=0.6$

Figure 4.12 Comparison of approximation formula and FEM for $T_{11}, a / W=0.8$

Figure 4.13 Mid-plane value of $T_{33}$ stress as a function of plate thickness in SECP, $a / W=0.2$ 
Figure 4.14 Mid-plane value of $T_{33}$ stress as a function of plate thickness in SECP,

$$
a / W=0.4 \text {.. }
$$

Figure 4.15 Mid-plane value of $T_{33}$ stress as a function of plate thickness in SECP, $a / W=0.6$

Figure 4.16 Mid-plane value of $T_{33}$ stress as a function of plate thickness in SECP, $a / W=0.8$

Figure 4.17 Comparison of approximation formula and FEM for $T_{33}, a / W=0.2$.

Figure 4.18 Comparison of approximation formula and FEM for $T_{33}, a / W=0.4$

Figure 4.19 Comparison of approximation formula and FEM for $T_{33}, a / W=0.6$.

Figure 4.20 Comparison of approximation formula and FEM for $T_{33}, a / W=0.8$.

Figure 4.21 Mid-plane value of stress intensity factor as a function of crack depth in SECP .122

Figure 4.22 Mid-plane value of $T_{11}$ as a function of crack depth in SECP. .122

Figure 4.23 Mid-plane value of $T_{33}$ as a function of crack depth in SECP. 123

Figure 4.24 Variations of relative SIF along the crack tip for plates various thickness, uniform, $a / W=0.2$ 123

Figure 4.25 Variations of relative SIF along the crack tip for plates various thickness, uniform, $a / W=0.4$ 124

Figure 4.26 Variations of relative SIF along the crack tip for plates various thickness, uniform, $a / W=0.6$ .124 
Figure 4.27 Variations of relative SIF along the crack tip for plates various thickness, uniform, $a / W=0.8$

Figure 4.28 Variations of relative $T_{11}$ along the crack tip for plates various thickness, uniform, $a / W=0.2$

Figure 4.29 Variations of relative $T_{11}$ along the crack tip for plates various thickness, uniform, $a / W=0.4$

Figure 4.30 Variations of relative $T_{11}$ along the crack tip for plates various thickness, uniform, $a / W=0.6$.

Figure 4.31 Variations of relative $T_{11}$ along the crack tip for plates various thickness, uniform, $a / W=0.8$

Figure 4.32 Variations of relative $T_{33}$ along the crack tip for plates various thickness, uniform, $a / W=0.2$

Figure 4.33 Variations of relative $T_{33}$ along the crack tip for plates various thickness, uniform, $a / W=0.4$ 128

Figure 4.34 Variations of relative $T_{33}$ along the crack tip for plates various thickness, uniform, $a / W=0.6$ 128

Figure 4.35 Variations of relative $T_{33}$ along the crack tip for plates various thickness, uniform, $a / W=0.8$

Figure 5.1 Coordinate system for stress state of crack front. 148

Figure 5.2 Stress State along the circular path close to the front at $z / t=0, a / W=0.8$, 
$t / W=0.1$

Figure 5.3 Stress State along the circular path close to the front at $z / t=0, a / W=0.8$, $t / W=0.2$

Figure 5.4 Stress State along the circular path close to the front at $z / t=0, a / W=0.8$, $t / W=0.5$

Figure 5.5 Stress State along the circular path close to the front at $z / t=0, a / W=0.8$, $t / W=1.0$

Figure 5.6 Stress State along the circular path close to the front at $z / t=0, a / W=0.8$, $t / W=2.0$

Figure 5.7 Stress State along the circular path close to the front at $z / t=0, a / W=0.8$, $t / W=4.0$

Figure 5.8 Percentage different of $f_{11}(\theta)$ varied by angles at three radiuses location, $a / W=0.8$, Uniform

Figure 5.9 Percentage different of $f_{22}(\theta)$ varied by angles at three radiuses location, $a / W=0.8$, Uniform: (a) Degree from $0^{\circ}$ to $135^{\circ}$, (b) Degree from $0^{\circ}$ to $165^{\circ}$ 152

Figure 5.10 Percentage different of $f_{33}(\theta)$ varied by angles at three radiuses location, $a / W=0.8$, Uniform

Figure 5.11 Stress State along the circular path close to the front varied by radius at four locations along thickness, $a / W=0.6, t / W=0.5, f_{11}$, Angle $=0^{\circ}$, Uniform, SECP.

Figure 5.12 Stress State along the circular path close to the front varied by radius at four locations along thickness, $a / W=0.6, t / W=0.5, f_{11}$, Angle $=30^{\circ}$, Uniform, SECP

$$
\mathrm{XIX}
$$


Figure 5.13 Stress State along the circular path close to the front varied by radius at four locations along thickness, $a / W=0.6, t / W=0.5, f_{l l}$, Angle $=60^{\circ}$, Uniform, SECP 154

Figure 5.14 Stress State along the circular path close to the front varied by radius at four locations along thickness, $a / W=0.6, t / W=0.5, f_{l l}$, Angle $=90^{\circ}$, Uniform, SECP........155

Figure 5.15 Stress State along the circular path close to the front varied by radius at four locations along thickness, $a / W=0.6, t / W=0.5, f_{11}$, Angle $=120^{\circ}$, Uniform, SECP......155

Figure 5.16 Stress State along the circular path close to the front varied by radius at four locations along thickness, $a / W=0.6, t / W=0.5, f_{l l}$, Angle $=150^{\circ}$, Uniform, SECP.....156

Figure 5.17 Stress State along the circular path close to the front varied by radius at four locations along thickness, $a / W=0.6, t / W=0.5, f_{11}$, Angle $=180^{\circ}$, Uniform, SECP......156

Figure 5.18 Stress State along the circular path close to the front varied by radius at four locations along thickness, $a / W=0.6, t / W=0.5, f_{22}$, Angle $=0^{\circ}$, Uniform, SECP .........157

Figure 5.19 Stress State along the circular path close to the front varied by radius at four locations along thickness, $a / W=0.6, t / W=0.5, f_{22}$, Angle $=30^{\circ}$, Uniform, SECP .......157

Figure 5.20 Stress State along the circular path close to the front varied by radius at four locations along thickness, $a / W=0.6, t / W=0.5, f_{22}$, Angle $=60^{\circ}$, Uniform, SECP .......158

Figure 5.21 Stress State along the circular path close to the front varied by radius at four locations along thickness, $a / W=0.6, t / W=0.5, f_{22}$, Angle $=90^{\circ}$, Uniform, SECP .......158

Figure 5.22 Stress State along the circular path close to the front varied by radius at four locations along thickness, $a / W=0.6, t / W=0.5, f_{22}$, Angle $=120^{\circ}$, Uniform, SECP .....159 
Figure 5.23 Stress State along the circular path close to the front varied by radius at four locations along thickness, $a / W=0.6, t / W=0.5, f_{22}$, Angle $=150^{\circ}$, Uniform, SECP .....159

Figure 5.24 Stress State along the circular path close to the front varied by radius at four locations along thickness, $a / W=0.6, t / W=0.5, f_{22}$, Angle $=180^{\circ}$, Uniform, SECP .....160

Figure 5.25 Stress State along the circular path close to the front varied by radius at four locations along thickness, $a / W=0.6, t / W=0.5, f_{33}$, Angle $=0^{\circ}$, Uniform, SECP 160

Figure 5.26 Stress State along the circular path close to the front varied by radius at four locations along thickness, $a / W=0.6, t / W=0.5, f_{33}$, Angle $=30^{\circ}$, Uniform, SECP .......161

Figure 5.27 Stress State along the circular path close to the front varied by radius at four locations along thickness, $a / W=0.6, t / W=0.5, f_{33}$, Angle $=60^{\circ}$, Uniform, SECP .......161

Figure 5.28 Stress State along the circular path close to the front varied by radius at four locations along thickness, $a / W=0.6, t / W=0.5, f_{33}$, Angle $=90^{\circ}$, Uniform, SECP .......162

Figure 5.29 Stress State along the circular path close to the front varied by radius at four locations along thickness, $a / W=0.6, t / W=0.5, f_{33}$, Angle $=120^{\circ}$, Uniform, SECP .....162

Figure 5.30 Stress State along the circular path close to the front varied by radius at four locations along thickness, $a / W=0.6, t / W=0.5, f_{33}$, Angle $=150^{\circ}$, Uniform, SECP .....163

Figure 5.31 Stress State along the circular path close to the front varied by radius at four locations along thickness, $a / W=0.6, t / W=0.5, f_{33}$, Angle $=180^{\circ}$, Uniform, SECP .....163

Figure 5.32 Percentage different varied by radius, $a / W=0.6, t / W=0.5$, Uniform, $0^{\circ} \ldots \ldots . .164$

Figure 5.33 Percentage different varied by radius, $a / W=0.6, t / W=0.5$, Uniform, $30^{\circ} \ldots . .164$ 
Figure 5.34 Percentage different varied by radius, $a / W=0.6, t / W=0.5$, Uniform, $60^{\circ} \ldots . .165$

Figure 5.35 Percentage different varied by radius, $a / W=0.6, t / W=0.5$, Uniform, $90^{\circ} \ldots . .165$

Figure 5.36 Percentage different varied by radius, $a / W=0.6, t / W=0.5$, Uniform, $120^{\circ} \ldots 166$

Figure 5.37 Percentage different varied by radius, $a / W=0.6, t / W=0.5$, Uniform, $150^{\circ} \ldots 166$

Figure 5.38 Stress State along the circular path close to the front varied by four locations along thickness, $a / W=0.6, t / W=0.1, r / a=0.0078, f_{11}$, Uniform, SECP

Figure 5.39 Stress State along the circular path close to the front varied by four locations along thickness, $a / W=0.6, t / W=0.1, r / a=0.0078, f_{22}$, Uniform, SECP

Figure 5.40 Stress State along the circular path close to the front varied by four locations along thickness, $a / W=0.6, t / W=0.1, r / a=0.0078, f_{33}$, Uniform, SECP 168

Figure 5.41 Stress State along the circular path close to the front varied by four locations along thickness, $a / W=0.6, t / W=0.2, r / a=0.0078, f_{l l}$, Uniform, SECP 168

Figure 5.42 Stress State along the circular path close to the front varied by four locations along thickness, $a / W=0.6, t / W=0.2, r / a=0.0078, f_{22}$, Uniform, SECP

Figure 5.43 Stress State along the circular path close to the front varied by four locations along thickness, $a / W=0.6, t / W=0.2, r / a=0.0078, f_{33}$, Uniform, SECP

Figure 5.44 Stress State along the circular path close to the front varied by four locations along thickness, $a / W=0.6, t / W=0.5, r / a=0.0078, f_{I l}$, Uniform, SECP 170

Figure 5.45 Stress State along the circular path close to the front varied by four locations 
along thickness, $a / W=0.6, t / W=0.5, r / a=0.0078, f_{22}$, Uniform, SECP

Figure 5.46 Stress State along the circular path close to the front varied by four locations along thickness, $a / W=0.6, t / W=0.5, r / a=0.0078, f_{33}$, Uniform, SECP

Figure 5.47 Stress State along the circular path close to the front varied by four locations along thickness, $a / W=0.6, t / W=1.0, r / a=0.0078, f_{l l}$, Uniform, SECP

Figure 5.48 Stress State along the circular path close to the front varied by four locations along thickness, $a / W=0.6, t / W=1.0, r / a=0.0078, f_{22}$, Uniform, SECP

Figure 5.49 Stress State along the circular path close to the front varied by four locations along thickness, $a / W=0.6, t / W=1.0, r / a=0.0078, f_{33}$, Uniform, SECP 172

Figure 5.50 Stress State along the circular path close to the front varied by four locations along thickness, $a / W=0.6, t / W=2.0, r / a=0.0078, f_{11}$, Uniform, SECP

Figure 5.51 Stress State along the circular path close to the front varied by four locations along thickness, $a / W=0.6, t / W=2.0, r / a=0.0078, f_{22}$, Uniform, SECP 173

Figure 5.52 Stress State along the circular path close to the front varied by four locations along thickness, $a / W=0.6, t / W=2.0, r / a=0.0078, f_{33}$, Uniform, SECP 174

Figure 5.53 Stress State along the circular path close to the front varied by four locations along thickness, $a / W=0.6, t / W=4.0, r / a=0.0078, f_{11}$, Uniform, SECP 174

Figure 5.54 Stress State along the circular path close to the front varied by four locations along thickness, $a / W=0.6, t / W=4.0, r / a=0.0078, f_{22}$, Uniform, SECP 175

Figure 5.55 Stress State along the circular path close to the front varied by four locations along thickness, $a / W=0.6, t / W=4.0, r / a=0.0078, f_{33}$, Uniform, SECP 175 XXIII 


\section{NOTATION}

$a$

$a_{n}, b_{n}, c_{n}, d_{n}, e_{n}$,

$f_{n}, g_{n}, h_{n}, i_{n}, j_{n}$

c

$f$

$f_{i i}$

$n$

$r, \theta$

$t$

$u_{i}$

$x, y, z$

$x_{1}, x_{2}, x_{3}$

$A_{l}$

$B^{2 D}$

$B_{33}^{2 D}$

$B^{\text {thin }}$

$B_{33}^{\text {thin }}$

$B_{i}$

$C_{i}$ crack depth

or half length of the major axis of elliptical cracks

or half length of the minor axis of quarter-elliptical cracks

parameters of $F_{n}$

half length of the minor axis of quarter-elliptical cracks

point force

functions for stress state near crack front

types of stress distribution

polar coordinates of plane normal to the crack front

plate thickness

components of displacement

Cartesian coordinate

Cartesian coordinate

parameters of $V_{0}$

or parameters of $W_{0}$

2D dimensionless "biaxiality" parameter

$2 \mathrm{D}$ dimensionless "biaxiality" parameter relative to $T_{33}$ stress

local "biaxiality" factor of a thin plate

local "biaxiality" factor of a thin plate relative to $T_{33}$ stress

parameters of $V_{1}$

or parameters of $W_{1}$

parameters of $V_{2}$

or parameters of $W_{2}$ 


$\begin{array}{ll}D_{i} & \text { parameters of } V_{3} \\ E & \text { or parameters of } W_{3} \\ F_{n} & \text { Young's Modulus } \\ G & \text { functions to represent the normalized } K_{\mathrm{I}} \\ \mathcal{G}_{c} & \text { strain energy release rate } \\ I(s) & \text { critical strain energy release rate } \\ J & \text { interaction integral } \\ K & J \text { integral } \\ K_{\text {surface }} & \text { stress intensity factor } \\ K_{u n c r a c k} & \text { stress intensity factor generated by crack pressure } \\ K_{I} & \text { stress intensity factor of uncracked specimen } \\ K_{I}^{2 D} & \text { stress intensity factor of opening mode } \\ K_{I I} & 2 \mathrm{D} \text { stress intensity factor of opening mode } \\ K_{I I I} & \text { stress intensity factor of shearing mode } \\ P & \text { stress intensity factor of tearing mode } \\ Q & \text { general point along the crack front } \\ T & \text { load system applied to the specimen } \\ T_{11} & T \text { stress } \\ T_{11}^{2 D} & T_{11} \text { stress } \\ T_{11 \text { surface }} & 2 \mathrm{D} T_{11} \text { stress } \\ T_{11 \text { uncrack }} & T_{11} \text { stress generated by crack pressure } \\ T_{12} & T_{11} \text { stress of uncracked specimen } \\ T_{22} & T_{12} \text { stress } \\ T_{33} & T_{22} \text { stress } \\ T_{33}^{2 D} & T_{33} \text { stress } \\ T_{33 \text { surface }} & 2 \mathrm{D} T_{33} \text { stress } \\ & \end{array}$




$\begin{array}{ll}T_{33 \text { uncrack }} & T_{33} \text { stress of uncracked specimen } \\ T_{2} & \text { out-of-plane constraint factor } \\ V_{n} & \text { functions to represent the normalized } T_{11} \text { stress } \\ V_{(s)} & \text { domain of interaction integral } \\ W & \text { plate width } \\ W_{n} & \text { functions to represent the normalized } T_{33} \text { stress } \\ \varepsilon_{i j} & \text { components of strain } \\ \mu_{k}(s) & \text { the direction normal to the crack front at point } s \\ \sigma_{0} & \text { uniform stress distribution } \\ \sigma_{i j} & \text { components of stresses } \\ \phi & \text { parametric angle } \\ v & \text { Poisson ratio } \\ \Delta K_{I 3 D} & \text { thickness correction factor of stress intensity factor } K_{\mathrm{I}} \\ \Delta T_{11_{3 D}} & \text { thickness correction factor of } T_{11} \text { stress } \\ \Delta T_{33_{3 D}} & \text { thickness correction factor of } T_{33} \text { stress }\end{array}$




\section{CHAPTER 1}

\section{INTRODUCTION}

Things break every day. A fracture can cost little, if only a broken toy, or a cracked automobile windshield. However, fractures can also lead to catastrophic failure and involve enormous expense. In 1988, the roof of the forward cabin of a 737 tore away during flight, a flight attendant was killed and many passengers were injured. The cause was multiple fatigue cracks linking up to form a large, catastrophic crack (Chandler, 1989). The accident challenged the notion that fracture was well understood and under control in modern structures.

The traditional straightforward design consideration to avoid structural failure is to keep the maximum stress well under the posted material strength. However, engineers found that most failure began with cracks from investigating fallen structures. Fracture mechanics was introduced to analyze the relationship among stresses, cracks, and fracture toughness. Fracture mechanics is a set of theories describing the behavior of solids or structures with geometrical discontinuity at the scale of the structure. The objective of fracture mechanics is to characterize the local deformation around a crack tip in terms of the asymptotic field around the crack tip scaled by parameters that are a function of the loading and global geometry.

Griffith (1921) set up the first milestone for fracture mechanics by his famous paper that quantitatively relates the flaw size to the fracture stresses. It presented that failure happened when stress level was far below the material strength when the tests were conducted on glass. However, Griffith's approach is only good for brittle materials.

Another milestone came out when Irwin $(1948,1958)$ developed the concept of strain energy release rate $\mathcal{G}$ for ductile materials. $\mathcal{G}$ is defined as the rate of change in potential energy near the crack area for a linear elastic material. The crack will grow 
when the strain energy release rate reaches the critical value $\mathcal{G}_{c}$. Later, stress intensity factor $K$ replaced the strain energy release rate $\mathcal{G} . K$ depends upon the applied stress, the size and placement of the crack, and the geometry of the specimen. $K$ is defined from the elastic stresses near the tip of a sharp crack under remote loading. And $K$ is used to predict the stress state near the tip of a crack.

The plasticity of the crack tips began to attract more attention from researchers after the fundamentals of fracture mechanics were established around 1960. Rice (1968) modeled the plastic deformation as nonlinear elastic behavior and extended the method of energy release rate to nonlinear materials. A path-independent line integral was established to express the energy release rate by Rice, called the $J$ integral. Meanwhile, a parameter called crack tip opening displacement (CTOD) led the fracture mechanics research in Europe. These methodologies are generally regarded to as the "one-parameter approach".

It is well known that the fracture resistance (toughness), which is the critical value of the crack driving force ( $K$ or $J$ ), is dependent on the constraint at the crack tip for a given cracked specimen. And the one-parameter fracture analysis is based on the fact that the crack has to be well constrained so that the minimum value of the material toughness can be achieved. This will ensure the fracture assessment is always in the safe side. But it may cause overly conservative assessment for the structures that are not well constrained, and thus raises the cost of product and maintenance.

Therefore, constraint-based failure analysis has been developed in order to describe the stress and strain fields ahead of a crack tip corresponding to different constraint levels. Rice (1974) suggested the $T$-stresses and stress intensity factor $K$ to be considered as the constraint parameter.

$T_{11}$ stress is defined as the second term of Williams (1957) series expansion of the stress component parallel to the crack plane. It can be used as the parameter to quantify the constraint effect. The Williams' series expanse the Mode $I$ stress field about 
a sharp crack in a linear elastic body with respect to $r$, the distance from the crack tip. Larsson and Carlsson (1973) and Rice (1974) showed that the magnitude of the $T$-stress affects the size and shape of the plastic zone and the region of tensile triaxiality ahead of the crack tip. If the $T_{11}$ stress is positive, $J$-dominance exists and a single parameter $J$ can be used for a fracture criterion. If the $T_{11}$ stress is negative, a two-parameter approach $\left(U\right.$ and $\left.T_{11}\right)$ is required to characterize the stress fields. Betegon and Hancock (1991), Du and Hancock (1991), O' Dowd and Shih (1991) and Wang (1993) indicated that the $T_{11}$ stress and $J$-integral provide effective two-parameters characterization of plane strain elastic-plastic crack tip fields in a variety of crack configuration and loading conditions.

For three-dimensional elastic crack problem, Rice (1974) suggested that both $T_{11}$ and $T_{33}$ stresses should be considered as the constraint parameters in order to characterize the stress field in the vicinity of crack front. $T_{33}$ stress is the second term of Williams (1957) series expansion of the stress component tangential along the crack front. Gonzalez-Albuixech et.al (2011) recently showed that the consideration of the components $T_{11}$ and $T_{33}$ was necessary to obtain a correct description of the stress state near the crack front.

The research of the three-dimensional solution of three parameters $K, T_{11}$ and $T_{33}$, and stress state around crack front have gained more attention recently. The thickness effective of the three parameters are also studied due to the various thickness in engineering application.

Raju and Newman (1977), Tracey (1974), Kwon and Sun (2000), and Kacinaauska et.al (2005) calculated the stress intensity factors by three-dimensional finite element analyses. The differences of the stress intensity factors between the three-dimensional along the crack front and two-dimensional were given.

Nakamura and Parks (1988, 1989, and 1992) analyzed the three-dimensional stress intensity factor and $T_{11}$ for thin elastic plate based on finite element analysis. The 
thickness effect of the $K$ and $T_{11}$ were described. Wang (2003), Wang and Bell (2004), and $\mathrm{Qu}$ and Wang (2006) presented the $T_{11}$ or $T_{33}$ stress for semi-elliptical surface or embedded elliptical cracks in a finite thickness plate under various loading conditions. Zhao et.al (2007) obtained the distributions of normalized $K$ and $T_{11}$ along the crack front for embedded elliptical cracks in elastic plates. And three-parameter $K-T_{11}-T_{z}$ describe the stress field around the crack front was provided with the corresponding out-of-plane constraint factor $T_{z}$. Meshii et. al (2010), Meshii and Tanaka (2010) performed both calculation and experimental for the thickness effect on the toughness affected by $T_{33}$.

The superposition method is a very powerful well-known tool for calculating stress intensity factor $K, T_{11}$ or $T_{33}$ stress under stress distributions. The superposition formula can be found in papers of Y. Yamamoto and co-workers (1973 and 1983), Wang and Bell (2004), Qu and Wang (2006) and Meshii et.al (2010).

The single edge cracked plate is a common specimen in research and practice of fracture mechanics. Most solutions are for two-dimensional plane-strain or plane-stress cases. However, some three-dimensional solutions of the $K, T_{11}$ or $T_{33}$ for the single edge cracked were studied but not systematic. Meanwhile, the thickness effect of $T_{33}$ is lack of research. The stress state near the crack front described by three-parameters need to be investigated as well.

The objectives of this thesis research are:

i). Systematic calculation of the fracture mechanics parameters $K_{I}, T_{11}$ and $T_{33}$ stresses for three-dimensional single edge cracked plate (SECP) specimen for wide range of parameter, relative crack depth $a / W$, relative thickness $t / W$ and four loading conditions (uniform, linear, parabolic and cubic) applied on the crack surface.

ii). Investigate the thickness effect $(t / W)$ on the three fracture mechanics parameters in SECP specimen with crack surface subjected to the uniform stress distribution.

iii). Study the accuracy of three parameter $K_{\mathrm{I}}-T_{11}-T_{33}$ characterizations of stress fields 
in three-dimensional SECP specimens under uniform loading condition.

\subsection{THESIS OUTLINE}

This thesis consists of six chapters. Chapter 1 is an introduction to the current research. In Chapter 2, an overview of the three-parameter definition, finite element method and superposition formula to calculate the stress intensity factor $K_{1}, T_{11}$ and $T_{33}$ stresses, and the stress state along the crack front are presented. Extensively literature review is also conducted in Chapter 2. The solutions of normalized stress intensity factor $K_{1}, T_{11}$ and $T_{33}$ stresses are derived in Chapter 3 for a single edged crack subjected to the various stress distribution in three-dimensional SECP specimens. And empirical equations of normalized stress intensity factor $K_{1}, T_{11}$ and $T_{33}$ stresses at the mid-plane are obtained. In Chapter 4 , the relative thickness and relative crack length effect of the mid-plane stress intensity factor $K_{1}, T_{11}$ and $T_{33}$ stresses are studied. And the stress intensity factor, $T_{11}$ and $T_{33}$ stresses variation along the crack front are presented. The stress state near the crack front described by stress intensity factor $K_{\mathrm{l}}, T_{11}$ and $T_{33}$ stresses are discussed in Chapter 5. Chapter 6 is the conclusion and recommendations of the thesis. 


\section{CHAPTER 2}

\section{BACKGROUND AND LITERATURE REVIEW}

In this chapter, the background on fracture mechanics parameters $K, T_{11}, T_{33}$, and the finite element method of obtaining them are summarized. Meanwhile, the superposition methods of calculating them are presented. Literature review is conducted on the calculation of $K, T_{11}$, and $T_{33}$ for three-dimensional cracked specimens.

\subsection{LINEAR ELASTIC FRACTURE MECHANICS}

\subsubsection{Fracture Modes and Stress Intensity Factor}

The linear elastic fracture mechanics was founded based on the theory of the stress intensity factor $K$, which was introduced by Irwin $(1948,1958)$. The objective of linear elastic fracture mechanics (LEFM) is to predict the critical loads that will cause a crack to grow in a brittle material. And the fundamental postulate of LEFM is that the value of the stress intensity factor determined the behavior of cracks. The stress intensity factors associated with three independent modes of movement of crack surfaces, opening mode $I\left(K_{l}\right)$, shearing mode $I I\left(K_{I I}\right)$, and tearing mode $I I I\left(K_{I I I}\right)$, see Figure 2.1 . The stress intensity factor may be used to determine the static strength of a cracked structure, the rate of growth of a crack in a structure subjected to variable loading, and the rate of growth of a crack in a loaded structure in a corrosive environment. Following Williams series expansion (Williams, 1957) for the stress state around the crack tip can be written as:

$$
\sigma_{i j}=r^{-\frac{1}{2}}\left\{K_{I} f_{i j}^{I}(\theta)+K_{I I} f_{i j}^{l \prime}(\theta)+K_{I I I} f_{i j}^{I \prime \prime}(\theta)\right\}+\text { higher order terms }
$$


where $\sigma_{i j}$ are the near tip stresses, and $K_{I}, K_{I I}, K_{I I I}$ are the stress intensity factors. Only mode $I$ stress intensity factor $K_{I}$ is considered in this thesis.

\subsubsection{T-Stress}

It has been realized that the ability of a single parameter such as the stress intensity factor to fully characterize crack tip conditions irrespective of geometry and load level is limited. Two parameters description of the crack tip stress-strain state have been studied. The next higher order, non-singular, stress terms in the Williams (1957) series expansion play an important role in crack behavior.

In an isotopic linear elastic body containing a crack subjected to symmetric (mode I) loading, the Williams (1957) series expansion for linear elastic crack-tip fields can be written as:

$$
\begin{aligned}
\sigma_{11} & =\frac{K_{l}}{\sqrt{2 \pi r}} \cos \frac{\theta}{2}\left(1-\sin \frac{\theta}{2} \sin \frac{3 \theta}{2}\right)+T_{11}, \\
\sigma_{22} & =\frac{K_{l}}{\sqrt{2 \pi r}} \cos \frac{\theta}{2}\left(1+\sin \frac{\theta}{2} \sin \frac{3 \theta}{2}\right), \\
\sigma_{33} & =\frac{K_{l}}{\sqrt{2 \pi r}} 2 v \cos \frac{\theta}{2}+T_{33}, \\
\sigma_{12} & =\frac{K_{l}}{\sqrt{2 \pi r}} \sin \frac{\theta}{2} \cos \frac{\theta}{2} \cos \frac{3 \theta}{2} \\
\sigma_{13} & =T_{13} \\
\sigma_{23} & =0 .
\end{aligned}
$$

where $r$ and $\theta$ are in-plane polar coordinates of plane normal to the crack front as shown in Fig.2.2, and $K_{l}$ is the local modal $I$ stress intensity factor at crack front. $v$ is the Poisson's ratio. The terms $T_{11}, T_{33}$ and $T_{13}$ are together called the $T$-stress. $T_{11}$ and $T_{33}$ are the amplitudes of the second order terms in the three-dimensional series expansion of the crack front stress field in the $x_{1}$ and $x_{3}$ directions, respectively. And only $T_{11}$ and $T_{33}$ are discussed in present work. 
From Williams' expansion, where higher order terms are neglected since the circular path is very close to the crack front, the following relationship is obtained:

$$
\frac{\sigma_{i l}-T_{i i}}{K_{l} / \sqrt{2 \pi r}}=f_{i i}(\theta) \quad i=1,2,3
$$

With

$$
\begin{aligned}
& f_{11}(\theta)=\cos \frac{\theta}{2}\left(1-\sin \frac{\theta}{2} \sin \frac{3 \theta}{2}\right) \\
& f_{22}(\theta)=\cos \frac{\theta}{2}\left(1+\sin \frac{\theta}{2} \sin \frac{3 \theta}{2}\right) \\
& f_{33}(\theta)=v\left(f_{11}(\theta)+f_{22}(\theta)\right)=2 v \cos \frac{\theta}{2}
\end{aligned}
$$

and $T_{22}=0$. That is the stress state described by $K_{l}, T_{11}$ and $T_{33}$.

Larsson and Carlsson (1973) and Rice (1974) showed that $T_{11}$ stress can substantially affect the size and shape of the crack-tip plastic zone. Cotterell and Rice (1980) showed that the sign of $T_{11}$ stress determines the stability of a straight mode I path is stable if $T<0$ and unstable if $T>0$. It means that positive $T_{11}$ stress strengthens the level of crack tip stress triaxiality and leads to high crack tip constraint, while negative $T_{11}$ stress reduces the level of crack tip stress triaxiality and leads to the loss the crack tip constraint.

The $T_{11}$ stress plays an important role in elastic-plastic fracture analysis, even though the $T_{11}$ stress is calculated from the linear elastic material properties of the same solid containing the crack.

Different methods to evaluate the $T_{11}$ stress results have been developed. Larsson and Carlsson (1973) and Wang and Parks (1992) and Coldthorpe (1992) used stress substitution methods to determine $T_{11}$ stress for three-dimensional cracked bodies. Leeves and Radon (1982) developed a computer program to determine the $T_{11}$ stress. Cardew et al. (1984) and Kfouri (1986) developed a method of calculating the $T_{11}$ stress for two-dimensional geometries based on an unpublished theorem due to Eshelby. The 
properties of the path-independent $J$-integral were used in this method. Sham (1991) developed a high accurate method of evaluating the $T_{11}$ stress for two-dimensional cracked geometries, which using so-called higher order weight functions evaluated by means of special integrals within a modified finite element procedure. Nakamura and Parks (1992) developed a method of evaluating $T_{11}$ stress for three-dimensional cracked structures which was essentially the 3D generalization of the Eshelby method. An interaction domain integral involving the stress, strain and displacement fields of the crack body subjected to its normal loading and those fields appropriate to a line load acting along the crack front was formulated. The local value of an interaction integral was used to derive the $T_{11}$ stress at any point of the crack. This method was used to evaluate the $T_{11}$ stress in ABAQUS (2008). The line spring method was used by Wang and Parks (1992) to develop a means of calculate the variation of $T_{11}$ stress along the crack front.

\subsubsection{Superposition Method for the determination of $K, T_{11}$ AND $T_{33}$}

Y. Yamamoto and co-workers (1983) developed a method of computing stress intensity factors based on the superposition of analytical and finite element solutions. The method was applied to problems of two-dimensional fracture, through the thickness cracks in plates subject to tension (Y. Yamamoto and N. Tokuda, 1973), and circumferentially cracked cylindrical bars subjected to constant tension and shear (Y. Yamamoto and Y. Sumi, 1974). Wang and Bell (2004) gave the formula to obtain $T_{11}$ stress based on superposition method. Qu and Wang (2006) and Meshii et.al (2010) introduced the formula for $T_{33}$ stress by superposition method.

The superposition method for the stress intensity factor and $T$-stresses calculation is discussed here. Consider a cracked two dimensional specimen loaded by mode $I$ load system $Q$, as demonstrated in Figure 2.3. The stress field of this problem can be divided into two parts: the regular field which appears under the same loading 
conditions in the uncracked specimen, and a corrective field due to the presence of the crack. The elastic stress intensity factor or $T$-stresses for the problem can be calculated from the summation of the stress intensity factor or $T$-stresses for these two problems, respectively.

The stress intensity factor for the crack specimen was given

$$
K=K_{\text {uncrack }}+K_{\text {surface }}
$$

Since the $K$ for the regular uncracked specimen is zero,

$$
K=K_{\text {surface }}
$$

$T_{11}$ stress for the cracked specimen was given

$$
T_{11}=T_{11 \text { uncrack }}+T_{11 \text { surface }}
$$

where $T_{11 \text { uncrack }}$ is the $T_{11}$ stress generated in the regular field and $T_{11 \text { surface }}$ is the $T_{11}$ stress generated by crack pressure. Applying the two-dimensional case of crack tip stress fields of equation, and the relationship between local and global coordinates, the corresponding $T_{11}$ stress for the regular field can be found to be

$$
T_{11 \text { uncrack }}=\lim _{r \rightarrow 0+}\left(\left.\sigma_{11}\right|_{\theta=0}-\left.\sigma_{22}\right|_{\theta=0}\right)=\left.\left(\sigma_{x}-\sigma_{z}\right)\right|_{x=a, z=0}
$$

where $\sigma_{x}\left(=\sigma_{11}\right)$ and $\sigma_{z}\left(=\sigma_{33}\right)$ are the stress components from the uncracked body under applied load system $Q$, and the location is at the crack tip. The $T_{11}$ stress for the problem is solved by substituting equation (2.8) into equation (2.7),

$$
T_{11}=\left.\left(\sigma_{x}-\sigma_{z}\right)\right|_{x=a, z=0}+T_{11 \text { surface }}
$$

For three-dimensional cracked plate, the $T_{11}$ is changing along the crack front, as shown in Figure 2.4. The counterpart of equation (2.7) is 


$$
T_{11}(P)=T_{11 \text { uncrack }}(P)+T_{11 \text { surface }}(P)
$$

where $P$ is the general point along the crack front. The uncracked $T_{11}$ is

$$
T_{11 \text { uncrack }}(P)=\left.\left[\lim _{r \rightarrow 0+}\left(\left.\sigma_{11}\right|_{\theta=0}-\left.\sigma_{22}\right|_{\theta=0}\right)\right]\right|_{P}=\left.\left(\sigma_{11}-\sigma_{22}\right)\right|_{P}
$$

The $T_{11}$ stress for the problem under consideration is

$$
T_{11}(P)=\left.\left(\sigma_{11}-\sigma_{22}\right)\right|_{P}+T_{11 \text { surface }}(P)
$$

Similarly, for $T_{33}$ stress

$$
T_{33}(P)=T_{33 \text { uncrack }}(P)+T_{33 \text { surface }}(P)
$$

By considering the $\theta=0$ plane, and by subtracting the singular term in $\sigma_{33}$ and $\sigma_{22}$ in Eq. (2.13), $T_{33}$ stress for the uncracked sub-problem is represented by

$$
T_{33 \text { uncrack }}(P)=\left.\left[\lim _{r \rightarrow 0+}\left(\left.\sigma_{33}\right|_{\theta=0}-\left.2 v \sigma_{22}\right|_{\theta=0}\right)\right]\right|_{P}=\left.\left(\sigma_{33}-2 v \sigma_{22}\right)\right|_{P}
$$

The $T_{33}$ stress for the problem under consideration is then

$$
T_{33}(P)=\left.\left(\sigma_{33}-2 v \sigma_{22}\right)\right|_{P}+T_{33 \text { surface }}(P)
$$

\subsection{FINITE ELEMENT ANALYSIS OF CRACKED COMPONETS}

In this section, the finite element method used in this thesis to calculate stress intensity factor $K_{l}, T_{11}$ and $T_{33}$ stresses is summarized. 


\subsubsection{Calculation of stress intensity factor $K_{I}$}

Several methods of estimating stress intensity factors are available, such as the alternating, weight function, body force, boundary element analysis and finite element analysis. The finite element method is employed in the simulation technique because of its versatility and generality for complicated cracked structures. Barsoum (1976) introduced a way to improve the numerical results in vicinity of the crack tip by using singular finite elements. One side of the element must be collapsed in order to have its three nodes just in one geometric point. The square-root stress singularity predicted by LEFM is exactly simulated by shifting the mid-side nodes to quarter-positions.

The $J$-integral, introduced by Rice (1968), is originally a two-dimensional line integral. It is used in rate-independent quasi-static fracture analysis to characterize the energy release associated with crack growth. It can be related to the stress intensity factor if the material response is linear and it also provides a method for analyzing fracture in nonlinear materials. The $J$-integral is path independent when contours are taken around a crack tip. It assumes that the material is homogeneous in the crack direction and the material is elastic.

The relation between the $J$ and the stress intensity factor for mode $I$ in a linear elastic material is

$$
\begin{array}{ll}
J=\frac{1-v^{2}}{E} K_{I}^{2} & \text { for plane strain } \\
J=\frac{1}{E} K_{I}^{2} & \text { for plane stress }
\end{array}
$$

The $J$-integral can also be generalized to three-dimensions by considering a tubular surface around the crack front. This domain integral method is used to evaluate contour integrals automatically in ABAQUS (2008). 


\subsubsection{Calculation of $T_{11}$ Stress}

Following is the method used to calculate $T_{11}$ stress which introduced by Nakamura and Parks (1992). Considering a three dimensional crack front with a continuously turning tangent as shown in Figure 2.2, assume a line-load of magnitude $f_{k}=f \mu_{k}(s)$ to be applied along the crack front as shown in Figure 2.5. $\mu_{k}(s)$ defines the direction normal to the crack front and in the plane of the crack at point $s$. The solution for this problem is the case of a plane strain semi-infinite crack with a point force $f$ applied at the crack tip in the direction parallel to the crack. Using superscript ' $L$ ' to designate the stress and displacement fields, the analytical solution gives (Timoshenko and Goodier, 1970)

$$
\begin{aligned}
& \sigma_{11}^{L}=-\frac{f}{\pi r} \cos ^{3} \theta, \quad \sigma_{22}^{L}=-\frac{f}{\pi r} \cos \theta \sin ^{2} \theta, \quad \sigma_{33}^{L}=-\frac{f}{\pi r} v \cos \theta, \\
& \sigma_{12}^{L}=-\frac{f}{\pi r} \cos ^{2} \theta \sin \theta, \quad \sigma_{13}^{L}=\sigma_{23}^{L}=0
\end{aligned}
$$

and

$$
\begin{aligned}
& u_{1}^{L}=-\frac{1-v^{2}}{E} \frac{f}{\pi}\left\{\ln \left(\frac{r}{d}\right)+\frac{\sin ^{2} \theta}{2(1-v)}\right\} \\
& u_{2}^{L}=-\frac{1+v}{2 E} \frac{f}{\pi}\{(1-2 v) \theta-\cos \theta \sin \theta\} \\
& u_{3}^{L}=0
\end{aligned}
$$

Employing the above solution as an auxiliary field, Cardew et al. (1984) and Kfouri (1986) extracted the $T_{11}$ stress for two-dimensional crack problems by introducing an interaction $J$ integral based on Eshelby's theorem. Nakamura and Parks (1992) extended this method to three-dimensional crack problems and provided the domain integral formulations. Combined with finite element method, it was shown to be a simple and effective method for the determination of the $T_{11}$ stress of two-dimensional and three-dimensional crack specimens. The interaction integral is introduced in the following 
domain integral form for three-dimensional crack problems,

$$
I(s)=\frac{1}{A_{c}} \oint\left\{\left(\sigma_{i j} \frac{\partial u_{i}^{L}}{\partial x_{k}}+\sigma_{i j}^{L} \frac{\partial u_{i}}{\partial x_{k}}\right) \frac{\partial q_{k}}{\partial x_{j}}-\varepsilon_{i j}^{L} \sigma_{i j} \frac{\partial q_{k}}{\partial x_{k}}\right\} d V
$$

The integration is in the domain $V_{(s)}$ which encloses the crack front segment between $s-\varepsilon$ and $s+\varepsilon, q_{k}(s)$ defines the virtual extension of the crack front segment $s-\varepsilon \ll s \ll s+\varepsilon$ and $A_{c}$ is the increase in crack area generated by the virtual crack advance; $\sigma_{i j}, \varepsilon_{i j}$ and $u_{i}$ are the stress, strain and displacement components of the three-dimensional crack problem under consideration; $\sigma_{i j}^{L}, \varepsilon_{i j}^{L}$ and $u_{i}^{L}$ are the corresponding components in the line-load auxiliary solution given by (2.17a) and (2.17b).

The crack tip $T_{11}$ stress is related to $I(s)$, the interaction integral, by the following:

$$
T_{11}(s)=\frac{E}{1-v^{2}}\left\{\frac{I(s)}{f}+v \varepsilon_{33}(s)\right\}
$$

This domain integral method is used to evaluate $T_{11}$ stress automatically in ABAQUS (2008).

\subsubsection{Calculation of $T_{33}$ Stress}

If the out-of-plane strain $\varepsilon_{33}$ is solved, an expression for calculating $T_{33}$ stress sufficiently far from the vertex corners can be obtained in a straightforward manner. The out-of-plane strain $\varepsilon_{33}$ at a given position of the crack front can be obtained by application of the Hooke's law for an isotopic material. 


$$
\varepsilon_{33}=\frac{1}{E}\left[\sigma_{33}-v\left(\sigma_{11}+\sigma_{22}\right)\right]
$$

After substitution of (2.2) in (2.20), obtain

$$
\varepsilon_{33}=\frac{1}{E}\left[2 v \frac{K_{\mathrm{I}}}{\sqrt{2 \pi r}}+T_{33}-v\left(\frac{K_{\mathrm{I}}}{\sqrt{2 \pi r}}+T_{11}+\frac{K_{\mathrm{I}}}{\sqrt{2 \pi r}}\right)\right]
$$

The following relationship is obtained

$$
\varepsilon_{33}=\frac{1}{E}\left[T_{33}-v T_{11}\right]
$$

It is shown that the out-of-plane strain $\varepsilon_{33}$ is dominated by second order terms that cannot be neglected at crack front.

$$
T_{33}=E \varepsilon_{33}+v T_{11}
$$

The value of $\varepsilon_{33}$ and $T_{11}$ have to be known in advance to calculate $T_{33}$.

\subsection{LITERATURE REVIEW FOR THREE-DIMENSIONAL SOLUTION OF $K, T_{11}$ AND $T_{33}$ AND STRESS STATE AROUND CRACK FRONT}

Earlier publications on three-dimensional cracks can be found in the review paper by Sih (1971). Raju and Newman (1977) used the force method and focused on obtaining stress intensity factor without the assumption of plane strain or plane stress at the crack tip. The stress intensity factor in the mid plane of center crack tension, single and double edge crack tension and compact specimens with through-the-thickness crack were calculated using a three dimensional finite element elastic stress analysis. The results were within $3 \%$ of the two-dimensional plane strain values.

Tracey (1974) built up an elastic three-dimensional finite element model for the through-the-thickness cracked compact tension specimen and calculated the mode I stress 
intensity factor along the crack front. The stress intensity factor variation with the thickness was discussed. It showed that the difference of the two and three-dimensional stress intensity factor solution was less than $1 \%$ in the region $0<z / t<0.28$, where $z$ is the axis along the thickness direction with original point at mid-plane of the specimen, and $t$ is the thickness. However, the differences greater than $10 \%$ only in the region front $z / t=0.44$ to the free surface $z / t=0.50$.

Kacianauskas et.al (2005) investigated the three-dimensional effects of the two-dimensional mode I stress intensity factor for the plate with a V-shaped straight through-thickness notch and gave a suggestion for three-dimensional thickness dependent correction of SIF.

Kwon and Sun (2000) performed a global-local finite element technique with sub-modeling to achieve the refinement required to obtain an accurate mode I stress field for plates with a through-the-thickness crack. The effect of plane thickness on the size of the three-dimensional region was studied and an approximate 3D stress intensity factor solution without $3 \mathrm{D}$ calculation at the mid-plane was suggested. A formula describes the relation between the 3D stress intensity factor at the mid-plane and the 2D stress intensity factor was given in that paper.

Nakamura and Parks (1988, 1989, and 1992) made great contributions for three-dimensional stress state analysis around the crack front in a thin elastic plate. Nakamura and Parks (1988) analyzed the three-dimensional of first stress intensity field near the crack front of a thin elastic plate based on very detailed full-field finite element analysis. Nakamura and Parks (1989) computed the antisymmetrical three-dimensional stress field in the same geometry. The asymptotic form of stress near the intersection under general loading conditions is presented, along with the corner stress intensity factor. $T_{11}$ stress distributions of single edge cracked plates and surface crack model in the plate are determined by finite element analysis. Nakamura and Parks (1992) gave an approximant formula for $T_{11}$ stress distributions along the single edge cracked plates in 
thin plane.

Henry and Luxmoore (1995) used thick three-dimensional finite element models of centre cracked plates to study the variation in the biaxiality factor $\beta$ with the crack aspect ratio $a / W$. The boundary layer method and the displacement field method were chosen to calculate the $T_{11}$ stress in three-dimensions. And it was shown that the boundary layer method gave results that compare rather well with the two-dimensional plane strain values than the displacement field method. It also presented that the material's Poisson's ratio and the specimen's thickness affect the three-dimensional evaluation of the biaxiality factor.

Gao (1992) analyzed the variation of $T_{11}$ and $T_{33}$ along a slightly wavy three-dimensional crack front. The results indicated that the $T_{11}$ increased with the curvature of the crack front but $T_{33}$ decreased with the crack front curvature.

Sherry et.al (1995) collected the published solutions for the $T_{11}$ stresses for a range of two and three-dimensional cracked geometries and presented some new results.

Wang and Bell (2004) evaluated the $T_{11}$ stresses solutions for a semi-elliptical surface crack fronts in a finite thickness plate under various loading conditions by using Nakamura and Parks' method. Qu and Wang (2006) also calculated the $T_{11}$ and $T_{33}$ stresses along a quarter-elliptical corner crack in a finite thickness plate subjected to tension and bending.

Meshii and Tanaka (2010) studied the CT specimen thickness effect on the $T_{33}$ stress, and an experimental expression for the thickness effect on the fracture toughness using $T_{33}$ stress is proposed for $0.55 \%$ carbon steel S55C. From their finite element results, it noted that small test specimen thickness dependence for the in-plane elastic parameters $K_{1}$ and $T_{11}$, and large dependence for the out-of-plane parameter $T_{33}$. And a suggestion of improving the existing methods for correlating fracture toughness obtained by test specimens with the toughness of actual cracks found in the structure using $T_{33}$ stress was given. Meshii et.al (2010) presented the $T_{11}$ and $T_{33}$ for semi-elliptical 
axial surface cracks in a cylinder subjected to mode I non-uniform stress on the crack surface. And the formulation of the superposition principle was given for the $T_{33}$ stress. Gonzalez-Albuixech et.al (2011) analyzed influence of the $T_{33}$ stress on the three-dimensional stress state around a mode I elliptical corner cracks with different aspect ratios in an elastic plate. It shows that the consideration of the components $T_{11}$ and $T_{33}$ is necessary to obtain a correct description of the stress state near the crack front. Wang (2003) calculated the elastic $T_{11}$-stress for semi-elliptical surface cracks in finite thickness plates by three dimensional finite element analyses. The $T_{11}$-stress solutions at the deepest, the surface and the middle points of the crack front under tension or bending were presented for cracks with relative depths $a / t$ values of $0.2,0.4,0.6$ or 0.8 and aspect ratio $a / c$ values of $0.2,0.4,0.6$ or 1.0 . The numerical results are approximated by empirical formulae fitted with an accuracy of $1 \%$ or better.

Yu and Wang (2005) presented the application of the weight function method for the calculation of elastic $T_{11}$-stress for semi-elliptical surface cracks. The weight function method was extended from two dimensional to three dimensional crack problem. And the $T_{11}$-stress weight functions for the deepest point and for any general point along the crack front of semi-elliptical surface cracks in finite-thickness plates for wide ranges of crack aspect radios $a / c$ and relative depths $a / t$ were derived.

$\mathrm{Qu}$ and Wang (2006) calculated the $T$-stresses ( $T_{11}$ and $T_{33}$ stress) for a quarter-elliptical corner crack in a finite thickness plate with a wide range of crack aspect ratios and relative depths. The loads were remote tension and bending. And empirical equations for $T$-stresses at two points near the free surfaces were also considered.

Zhao et.al (2007) obtained the distributions of normalized stress intensity factor and $T_{11}$ stress along the crack front with aspect ratios of $0.2,0.4,0.5,0.6,0.8$ and 1.0 for embedded elliptical cracks in elastic plates. And far-field tension and the effect of Poisson's ratio were also considered. Empirical formulae were given for the convenience of engineering applications. Combining with the corresponding out-of-plane constraint 
factor $T_{z}$, the three-parameter $K-T_{11}-T_{z}$ describe the stress field around the crack front was provided.

In this thesis, continuation of these early works is carried out. In particular, the three-dimensional single edge cracked plate (SECP) specimens are investigated.

\subsection{SINGLE EDGE CRACKED PLATE SPECIMEN}

The single edge cracked plate (SECP) is a common specimen in research and practice of fracture mechanics, see Figure 2.6. The most common test specimen configurations are single edge notched bend s (SENB or three-point bend), and the compact tension (CT) specimens.

The single edge notched bend (SENB) specimen is one of specimens that are permitted in ASTM standards that characterize fracture initiation and crack growth. There are three important characteristic dimensions for SENB specimen: the crack depth $(a)$, the thickness $(t)$ and the width $(W)$. In ASTM standard test, $W=2 t$ and $a / W=0.5$. The SENB specimens have been performed vast majority of fracture toughness tests.

The most tests for SENB specimen are three-point bending, which is high constraint test. But the low constraint test can be applied on the SENB specimen by changing $t / W, a / W$, and loading condition.

The simulation of crack parameters for the single edge cracked plate specimen is most focus on the stress intensity factor $K$ and $T_{11}$ stress. There are some solutions for two and three dimensional geometries. And a few researchers analyzed the thickness effect of the stress intensity factor and $T_{11}$ stress for single edge cracked plate specimen. The three-dimensional of $T_{33}$ stress distribution and the stress state described by three parameters (stress intensity factor, $T_{11}$ and $T_{33}$ stress) near the crack front are not studied systematically. Next, the available solutions are reviewed. 


\subsubsection{Two dimensional solutions for single edge cracked plate specimen}

Gross et al. (1965) calculated the stress intensity factor for single edge tension notch, three point bend and compact tension fracture specimens. Fett (1998) provided the solutions of stress intensity factor and $T_{11}$ stress for rectangular plates with various length-to-width ratios and different types of cracks (single and double-edge cracks) by the boundary collocation method. Sham (1991) gave the numerical values of the elastic $T_{11}$ stress and stress intensity factor for single edge notch specimens under tension, pure bending and three-point bend by using higher order weight functions. Wang (2002) calculated elastic $T_{11}$ stress solutions for cracked test specimens by finite element analysis. And the $T_{11}$ stress solutions were presented for single edge cracked plates, double edge crack plates and centre cracked plates. The stress distributions applied to the crack face were uniform, linear, parabolic or cubic.

\subsubsection{Three dimensional solutions for single edge cracked plate specimen and the thickness effect}

Some researchers gave the three dimensional solutions of stress intensity factor and $T$-stresses for single edge cracked plate specimen. Meantime, the thickness effect for stress intensity factor or $T_{11}$ stress was studied.

Kwon and Sun (2000) analyzed the effect of plane thickness on the size of the three-dimensional region and suggested an approximate 3D stress intensity factor solution without 3D calculation at the mid-plane for through thickness crack. It showed that the 3D stress intensity factor at the mid-plane can obtain front the 2D stress intensity factor with the relation

$$
K_{I 3 D} / K_{I 2 D}=\sqrt{1 /\left(1-v^{2}\right)}
$$


where $v$ is the Poisson's ratio.

Kacianauskas et.al (2005) assumed the thickness influence is characterized by the single stress intensity parameter $K_{I 3 D}(z / t)$, which is thickness-dependent variable. The three dimensional stress intensity factor can be presented as two dimensional value corrected by the thickness influence

$$
K_{I 3 D}(z / t)=K_{I 2 D} \Delta K_{I 3 D}(z / t)
$$

where $z$ is the axis along the crack front, $t$ is the thickness of the plate, and $\Delta \mathrm{K}_{\mathrm{I3D}}(z / t)$ is the thickness correction factor .

Nakamura and Parks (1992) studied the stress intensity factor and $T_{11}$ stress affected by Poisson's ratio, crack length, and plate thickness for single edge cracked plates in thin plane. An approximant formula to predict $T_{11}$ stress distributions along the cracked front in thin plane was given. The following is the detail about the formula.

From the finite element results of the $T_{11}$ stress for single edge cracked plate, it is noticed that when the thickness $t$ became large compared to in-plane dimensions (e.g. crack lengtha), the thickness-dependence of the $T_{11}$ stress distribution merge to two-dimensional solution, so $T_{11}$ can be expressed as

$$
\begin{aligned}
& T_{11}(z / t, v)=B^{2 D} \frac{K_{I}^{2 D}}{\sqrt{\pi a}} \equiv T_{11}^{2 D} \\
& \text { as } t / a \rightarrow \infty
\end{aligned}
$$

where $B^{2 D}$ is a dimensionless "biaxiality" parameter depending only on in-plane geometry and loading. Larsson and Carlsson (1973), Leevers and Radon (1983), Cardew et al. (1984), Kfouri (1986) and Sham (1991) gave the values of $B^{2 D}$ for several crack geometries.

It is proposed that, from considerations of superposition and dimensional analysis, as relative thickness diminished in the boundary value problem posed, the through-thickness variation of the $T_{11}$ stress distribution can be expressed as, 


$$
T_{11}(z / t, v) \cong T_{11}^{2 D}+B^{t h i n}(z / t, v) \frac{K_{l}(z / t, v)}{\sqrt{\pi t}}
$$

where the $T_{11}^{2 D}$ is the two-dimensional solution. Here $T_{11}^{2 D}$ represents the "remote" value which applies in the plane stress field found at radii large compared to thickness $t$, but small compared to crack length $a$. The $B^{\text {thin }}$ represents the local "biaxiality" factor of a thin plate. The functional form of (2.27) is motivated from the perspective of small relative thickness. However, a broader range of applicability is anticipated since the thick plate result (2.26) is recovered as $t / a \rightarrow \infty$ at fixed in-plane dimensions. It also notes that the normalized $T_{11}$ stress decreases and approaches the two-dimensional solution as the plate thickness increases from the finite element solutions.

Then when the thickness is large, Eq.(2.25) can be written as,

$$
\begin{gathered}
T_{11}(z / t, v)=B^{2 D}(z / t, v) \frac{K_{l}^{2 D}(z / t, v)}{\sqrt{\pi a}} \equiv T_{11}^{2 D} \\
\text { as } \quad t / a \rightarrow \infty
\end{gathered}
$$

Nakamura and Parks (1992) propose that locally there exists a $T_{11}$ stress distribution along the thin plate crack front based on dimensional grounds, the formula of the $T_{11}$ stress distribution is

$$
\begin{gathered}
T_{11}(z / t, v)=B^{\text {thin }}(z / t, v) \frac{K_{\mathrm{I}}(z / t, v)}{\sqrt{\pi t}} \\
t / a \rightarrow 0
\end{gathered}
$$

as

With the use of Eq. (2.26), (2.27), (2.28) and (2.29), the normalized $T_{11}(z / t, v)$ stress can be expressed as

$$
\frac{T_{11}(z / t, v)}{K_{l}(z / t, v) / \sqrt{\pi t}} \cong \sqrt{\frac{t}{a}} B^{2 D}+B^{\operatorname{thin}}(z / t, v)
$$

The first term on the right-hand side of Eq. (2.30) dominates for a thick plate, while it vanishes for a thin plate. The mid-plane value of $B^{\text {thin }}(0, v=0.3)$ was given as 0.045 . 


\subsubsection{The present research for single edge cracked plate}

In summary, there are three main reasons to perform present research for single edge cracked plate:

i). No systemic study for three dimensional stress intensity factor and $T$-stresses ( $T_{11}$ and $T_{33}$ stress). Former research was either focused on two dimensional geometry or limited relative crack length and relative thickness for three dimensional geometries.

ii). The thickness effect is not investigated thoroughly. Especially $T_{33}$ stress variation along the crack front for single edge cracked plate was lack of study.

iii). The three parameters control the stress state around the crack front of the single edge cracked plate need to be analyzed.

The stress intensity factor and $T$-stresses ( $T_{11}$ and $T_{33}$ stress) for different crack depth and thickness of the single edge cracked plate are calculated by finite element analysis in Chapter 3. The thickness effect and the stress state around the crack front described by three parameters are also studied in Chapter 4 and 5 . 


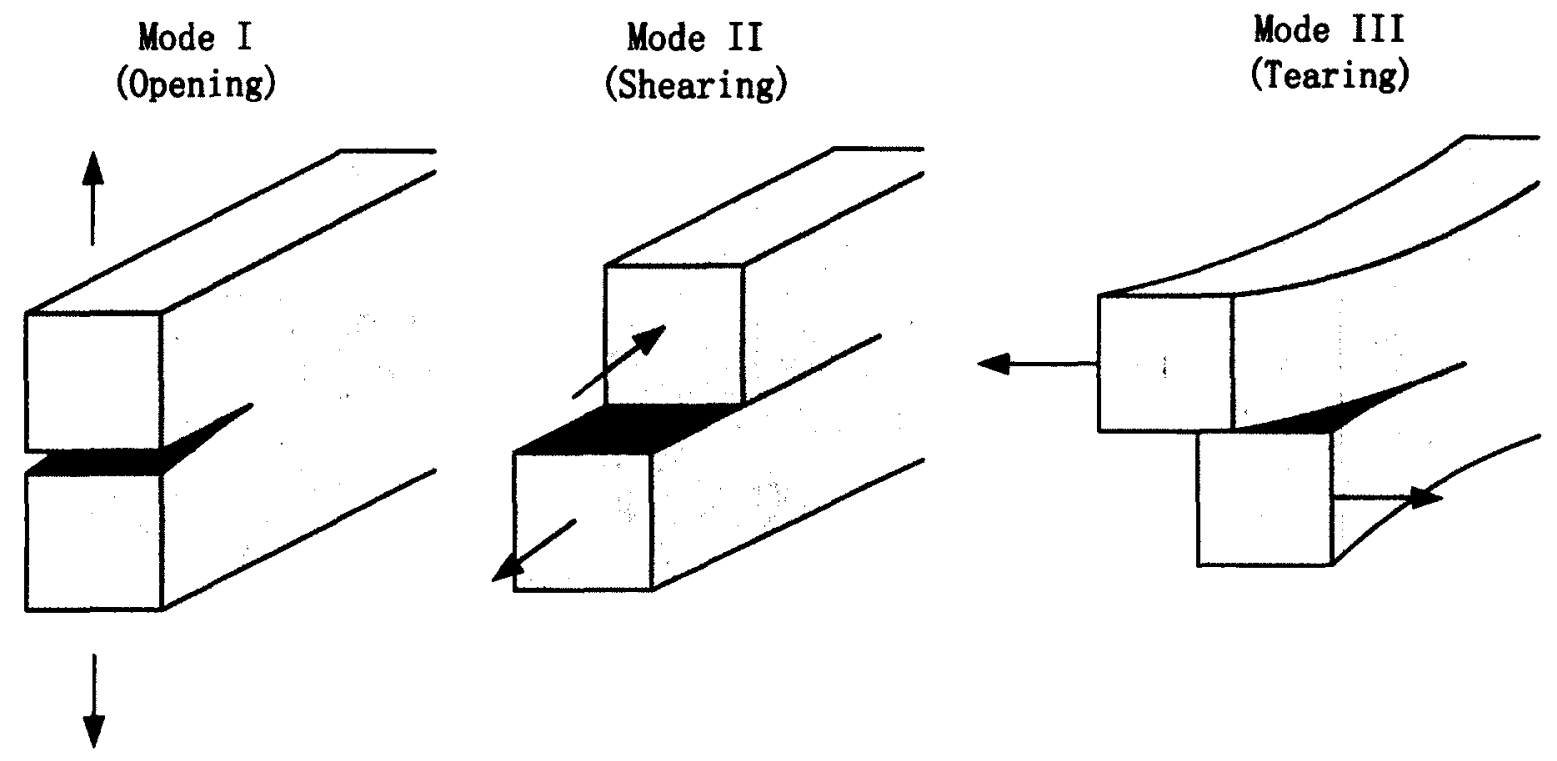

Figure 2.1 The three independent modes of movement of crack surfaces

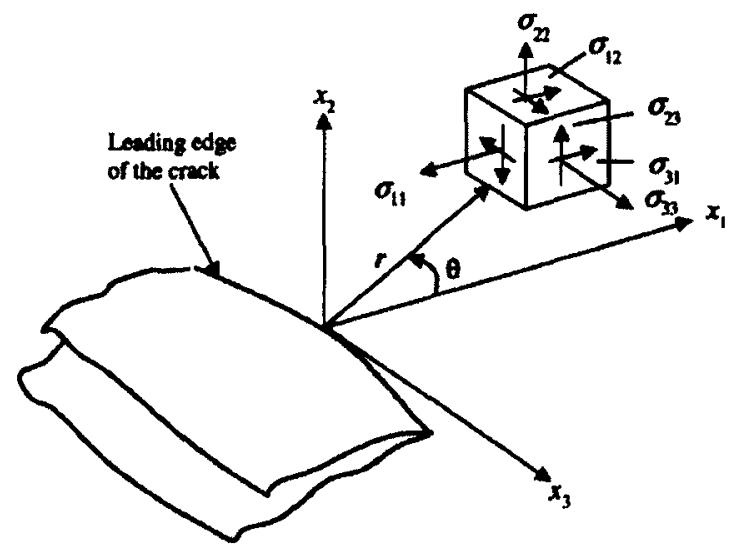

Figure 2.2 Three-dimensional coordinate system for the region along the crack front 


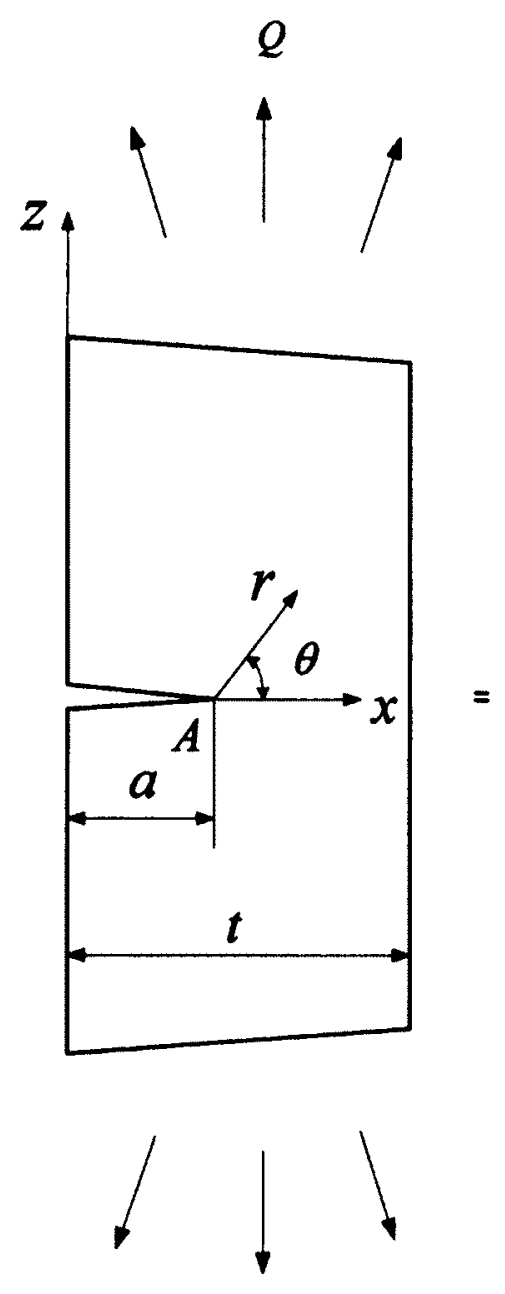

(a)
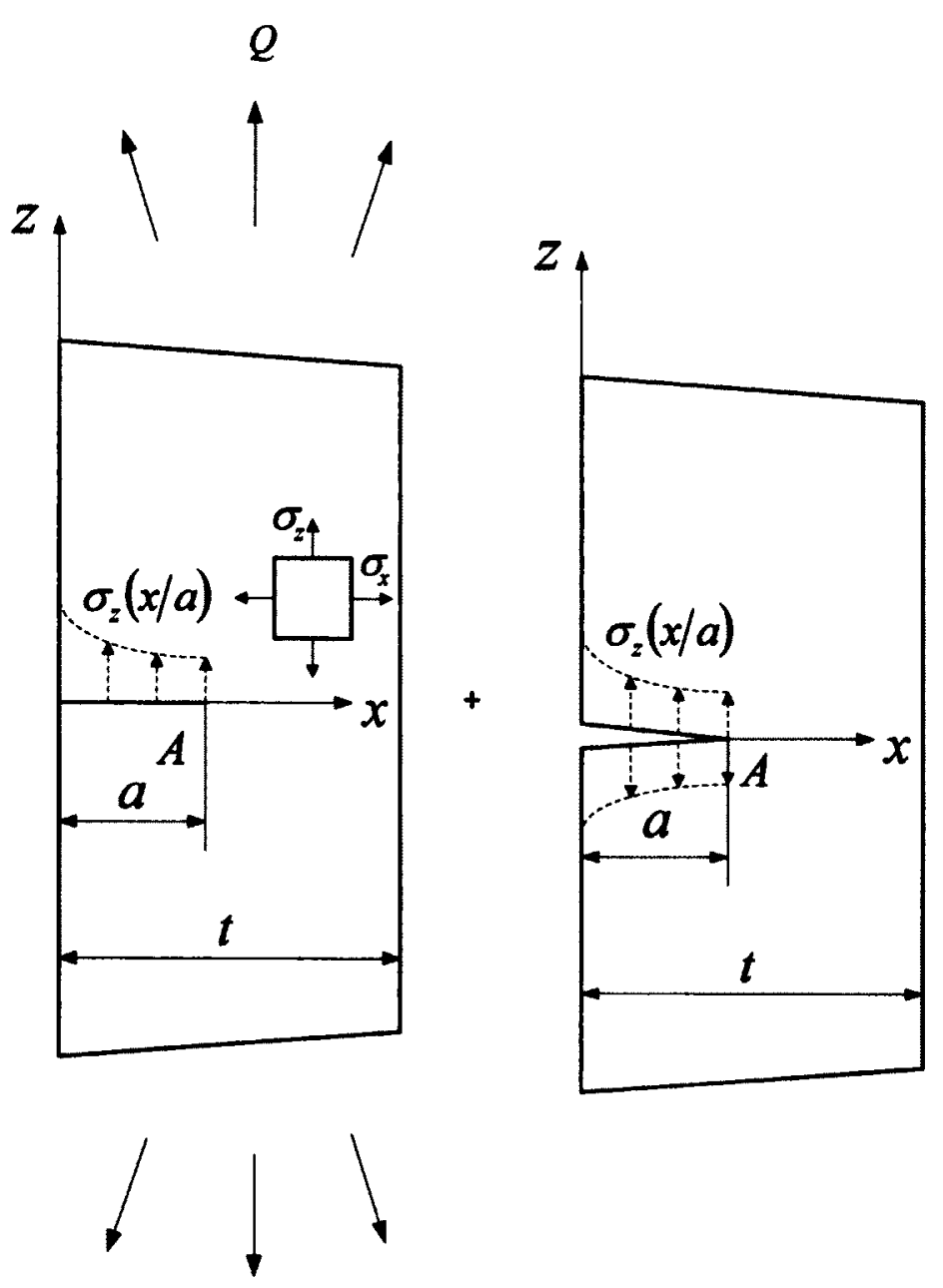

(b) (c)

Figure 2.3 Principle of superposition, two-dimensional 


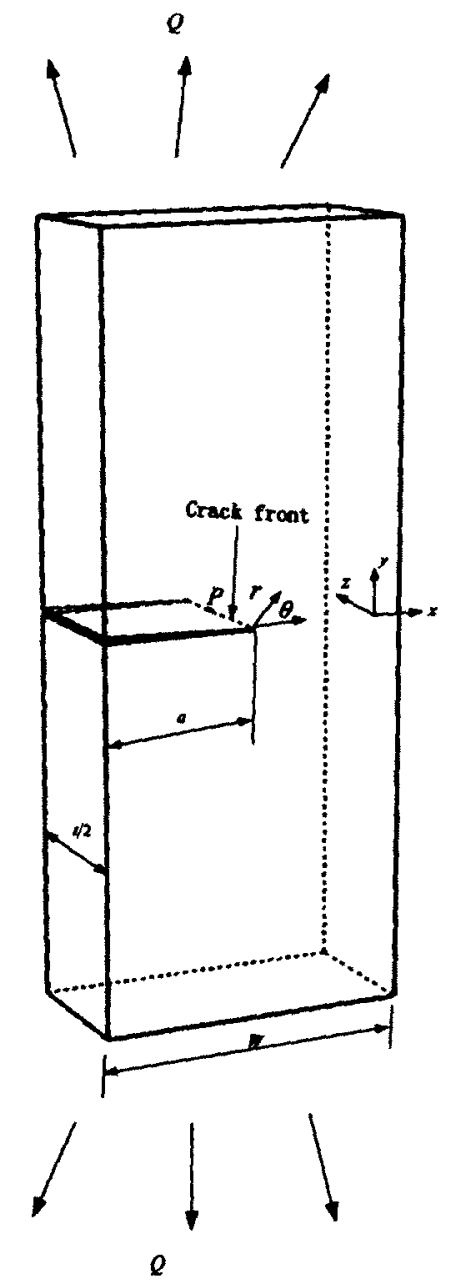

(a)

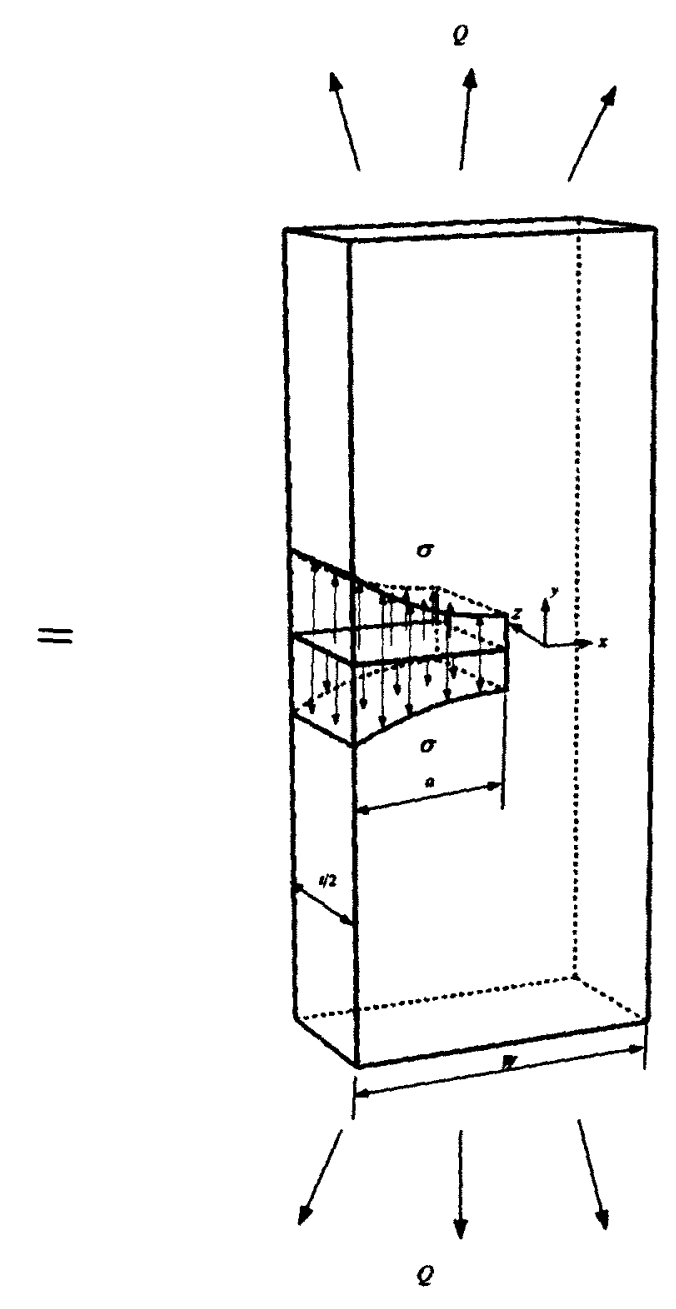

(b)

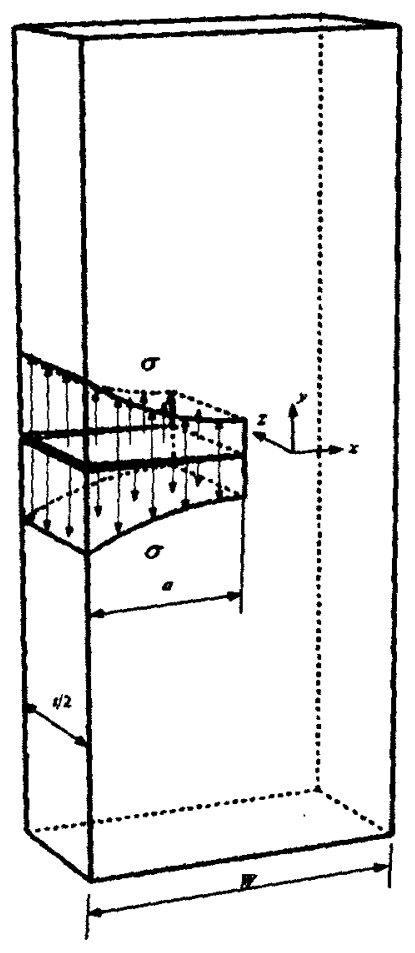

(c)

Figure 2.4 Principle of superposition, three-dimensional 


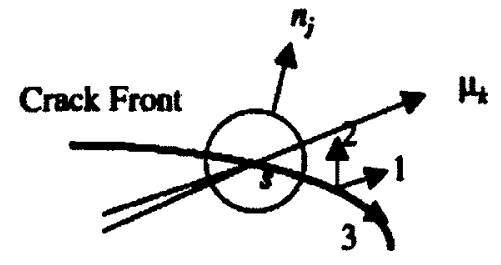

(a)

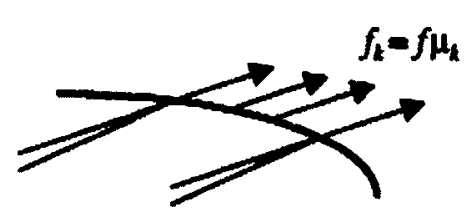

(b)

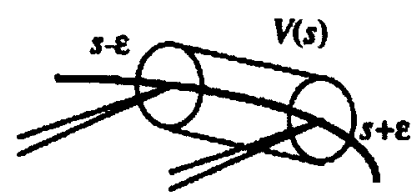

(c)

Figure 2.5 A schematic of elements used in the definition of the interaction integral: (a), (b), (c)

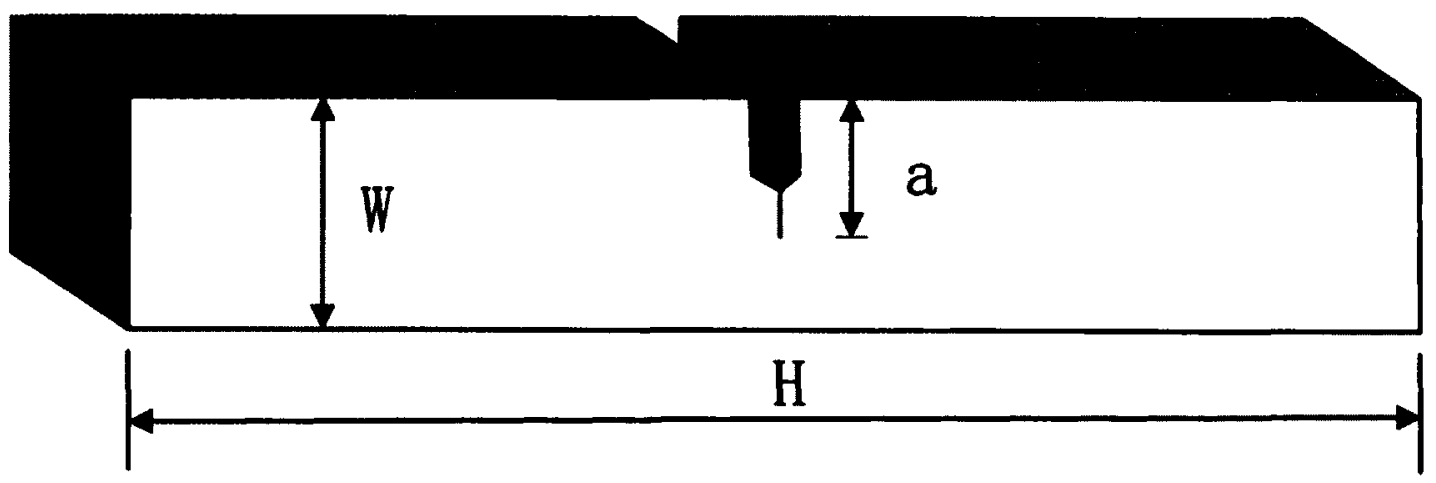

Figure 2.6 Single edge cracked specimen 


\section{CHAPTER 3}

\section{STRESS INTENSITY FACTOR AND T-STRESSES FOR CRACK IN THREE DIMENSIONAL SINGLE EDGE CRACKED PLATE SPECIMENS}

In this chapter, three-dimensional finite element analyses are conducted to obtain the stress intensity factor $K_{1}$ and $T$-stresses $\left(T_{11}\right.$ and $\left.T_{33}\right)$ for three-dimensional single edge cracked plate (SECP) specimens for wide range of $a / W$ and $t / W$ ratios to investigate the effect of thickness on these fracture parameters. And empirical equations of normalized stress intensity factor $K_{1}, T_{11}$ and $T_{33}$ stresses at the mid-plane are obtained for engineering application.

\section{$\begin{array}{llllll}3.1 & \text { FINITE } & \text { ELEMENT } & \text { ANALYSIS FOR } & \text { CRACK IN }\end{array}$} TRHEE-DIMENSIONAL SINGLE EDGE CRACKED PLATE SPECIMENS

In this section, the three-dimensional finite element model to calculate the three fracture parameters are created and verified. The normalized stress intensity factor $K_{1}$, $T_{11}$ and $T_{33}$ stresses along the crack front for different relative crack depth, $a / W$, and relative thickness, $t / W$ for SECP specimen with crack surface subjected to different stress distributions are obtained.

\subsubsection{Finite Element Model}

Three-dimensional model of single edge cracked plate (SECP) specimen was built in ABAQUS (2008). The geometry of the specimen is as shown in Figure 3.1. In the 
model, the ratio of half-height, $H$, to the width, $W$, was chosen to be $H / W=3$. Geometries through variations in crack depth, $a$, and plate thickness, $t$, were considered. The Poisson's ratio was 0.3 and Young's module was $206 \mathrm{GPa}$. The values of relative crack depth $a / W$ were $0.2,0.4,0.6$ and 0.8 , respectively. The values of $t / W$ were chosen as $0.1,0.2,0.5,1.0,2.0$ and 4.0 , respectively.

A quarter of the plate model was built to apply finite element analysis in ABAQUS (2008). The symmetry conditions across the mid-plane $(z / t=0)$ and the crack, and ligament plane $(y / t=0)$ were used in constructing the finite element mesh. Thinner elements were employed away from the mid-plane due to a high stress gradient along the crack front was expected near the free-surface. The number of element layers through the half-thickness was 20 for $t / W=0.1,30$ for $t / W=0.2$, and 40 for $t / W=0.5$, $1.0,2.0$ and 4.0 , respectively.

The 20-noded three-dimensional quadratic elements with reduced integration (C3D20R) were used to construct the quarter-model in ABAQUS (2008). In order to model the square root singularity at the crack tip, three-dimensional prism elements with four midside nodes at the quarter points (a degenerate cube with one face collapsed) were used. The C3D20R element and mesh configuration near the crack tip are shown in Figure 3.2

The loads were applied directly to the crack face, as shown in Figure 3.1. The stress intensity factor and $T$-stresses $\left(T_{11}\right.$ and $\left.T_{33}\right)$ were calculated for the following stress fields:

Uniform stress field

$$
\sigma(x)=\sigma_{0}
$$

Linear stress field

$$
\sigma(x)=\sigma_{0}\left(1-\frac{x}{a}\right)
$$


Parabolic stress field

$$
\sigma(x)=\sigma_{0}\left(1-\frac{x}{a}\right)^{2}
$$

Cubic stress field

$$
\sigma(x)=\sigma_{0}\left(1-\frac{x}{a}\right)^{3}
$$

A finite element model for $a / W=0.4, t / W=0.5$, single edge cracked plate specimen is shown as Figure 3.3.

The stress intensity factors along crack front were calculated by J-integral (energy release rate) method. The calculation of $T_{11}$ stress was based on Nakamura and Parks' (1992) interaction integral method. This technique was discussed in section 2.4.2. Five contours were used in the current analysis, the J-integral was stable after two contours, and the average value of $T_{11}$ results from the last two outer contours was used to calculated crack $T_{11}$ distributions. $T_{33}$ was calculated by the Eq.2.23.

\subsubsection{Verification of the Finite Element Model}

In order to verify the finite element model and analysis method for calculating stress intensity factor $K_{1}, T_{11}$ and $T_{33}$ stresses, a surface corner crack model $(a / c=1.0$, $a / t=0.2)$ in plate was built in ABAQUS (2008). The geometry of surface corner crack is shown in Figure 3.4 and the mesh is shown in Figure 3.5.

The results of $K_{1}, T_{11}$ and $T_{33}$ compared with Qu and Wang's (2006) results are shown in Table 3.1-3.3, respectively. The differences of stress intensity factor $K_{\mathrm{I}}$ for two positions $\left(0^{\circ}\right.$ and $\left.90^{\circ}\right)$ between present analysis and $\mathrm{Qu}$ and Wang's results are $2.39 \%$ and 3.24\%, respectively. There is a good agreement for $T_{11}$ stress solutions along the crack front for present analysis and Qu and Wang's model. Most of the $T_{11}$ stress results 
differences are less than 3\%, except the point of $5^{\circ}$ is $3.12 \%$, as shown in Figure 3.6. It shows that the most difference of $T_{33}$ between present research and $\mathrm{Qu}$ and Wang's result is less than $3 \%$, except the difference at the point near the deepest point is $7.17 \%$, as shown in Figure 3.7.

In order to verify the finite element model of single edge cracked plate (SECP) specimen, a model was built in ABAQUS (2008) which the relative crack depth and thickness were fixed at $a / W=0.5$ and $t / W=0.5$, respectively. Remote tension loading was applied to the specimen. Poisson ratio $v$ was chosen from $0,0.3$ and 0.499 , respectively, and the straight crack front intersects the free-surfaces orthogonally. This was the geometry analyzed by Nakamura and Parks (1992).

The results of $T_{11}$ for the tension case are shown in Table 3.4 and plotted in Figure 3.8. The initial data and trends are good fit with the results of Nakamura and Parks (1992). And the results of stress intensity factor $K_{\mathrm{I}}$ and $T_{11}$ for $v=0$ (or the two-dimensional solution) were compared with Sham's results (1991), as Table 3.5. The differences are within $0.11 \%$ for stress intensity factor $K_{\mathrm{I}}$ and $1.91 \%$ for $T_{11}$ stress.

Through these calculations, the present finite element procedures are considered appropriate for $K_{\mathrm{l}}, T_{11}$ and $T_{33}$ calculations for three-dimensional SECP specimens.

\subsubsection{Finite Element Results}

The stress intensity factor $K_{1}$ and $T$-stresses $\left(T_{11}\right.$ and $\left.T_{33}\right)$ values for cracks in SECP specimens with $a / W$ of $0.2,0.4,0.6$ or 0.8 , and $t / W$ of $0.1,0.2,0.5,1.0,2.0$, or 4.0 , subjected to constant, linear, parabolic or cubic stress distribution as expressed in Eq. (3.1-3.4) have been determined.

The stress intensity factor $K_{I}$ and $T$-stresses $\left(T_{11}\right.$ and $\left.T_{33}\right)$ results have been normalized as follows: 


$$
\begin{aligned}
& \text { Normalized } K_{I}=\frac{K_{I}}{\sigma_{0} \sqrt{\pi a}} \\
& \text { Nomalized } T_{11}=\frac{T_{11}}{\sigma_{0}} \\
& \text { Nomalized } T_{33}=\frac{T_{33}}{\sigma_{0}}
\end{aligned}
$$

The results of normalized stress intensity factors $K_{I}$ and $T$-stresses $\left(T_{11}\right.$ and $T_{33}$ ) are summarized in Table 3.6-3.11 for $a / W=0.2$. The normalized stress intensity factors $K_{I}$ for $a / W=0.2$ cases subjected to constant or linear are shown in Figure 3.9 and 3.10 , respectively. The normalized $T_{11}$ for $a / W=0.2$ cases subjected to constant or linear are shown in Figure 3.11 and 3.12, respectively. And the normalized $T_{33}$ for $a / W=0.2$ cases subjected to constant or linear are shown in Figure 3.13 and 3.14, respectively.

The normalized stress intensity factors $K_{1}$ and $T$-stresses $\left(T_{11}\right.$ and $\left.T_{33}\right)$ for $a / W=0.4$ are summarized in Table 3.12-3.17, and the stress intensity factors $K_{I}$ for the cases subjected to constant or linear are shown in Figure 3.15 and 3.16, respectively. The normalized $T_{11}$ for $a / W=0.4$ cases subjected to constant or linear are shown in Figure 3.17 and 3.18 , respectively. And the normalized $T_{33}$ for $a / W=0.4$ cases subjected to constant or linear are shown in Figure 3.19 and 3.20, respectively.

The results of normalized stress intensity factors $K_{\mathrm{I}}$ and $T$-stresses $\left(T_{11}\right.$ and $\left.T_{33}\right)$ are summarized in Table 3.18-3.23 for $a / W=0.6$. The normalized stress intensity factors $K_{\mathrm{I}}$ for $a / W=0.6$ cases subjected to constant or linear are shown in Figure 3.21 and 3.22, respectively. The normalized $T_{11}$ for $a / W=0.6$ cases subjected to constant or linear are shown in Figure 3.23 and 3.24, respectively. And the normalized $T_{33}$ for $a / W=0.6$ cases subjected to constant or linear are shown in Figure 3.25 and 3.26, respectively.

The normalized stress intensity factors $K_{1}$ and $T$-stresses $\left(T_{11}\right.$ and $\left.T_{33}\right)$ for $a / W=0.8$ are summarized in Table 3.24-3.29, and the stress intensity factors $K_{l}$ for the cases subjected to constant or linear are shown in Figure 3.27 and 3.28, respectively. The normalized $T_{11}$ for $a / W=0.8$ cases subjected to constant or linear are shown in Figure 
3.29 and 3.30 , respectively. And the normalized $T_{33}$ for $a / W=0.8$ cases subjected to constant or linear are shown in Figure 3.31 and 3.32, respectively.

\subsection{EMPIRICAL EQUATIONS OF STRESS INTENSITY FACTOR AND T-STRESS}

For important engineering applications, the numerical stress intensity factor $K_{\mathrm{I}}$ and $T$-stresses results for cracks in SECP at position $z / t=0$ presented in Section 3.1 were approximated by empirical formulae fitted. These empirical formulae are least square fitted using results presented in Tables 3.6-3.29.

\subsubsection{Stress Intensity Factor Equations}

The stress intensity factor $K_{I}$ solutions at $z / t=0$ for stress applied on the crack front corresponding to the Eq. (3.1) to (3.4) are expressed in the following forms:

$$
\begin{aligned}
K_{\mathrm{I}}=\sigma_{0} F_{n}\left(\frac{a}{W},\right. & \left.\frac{t}{W}\right) \sqrt{\pi a} \\
F_{n}=a_{n}+b_{n} & \ln \left(\frac{t}{W}\right)+c_{n}\left(\frac{a}{W}\right)+d_{n}\left(\ln \left(\frac{t}{W}\right)\right)^{2}+e_{n}\left(\frac{a}{W}\right)^{2}+f_{n}\left(\frac{a}{W}\right) \ln \left(\frac{t}{W}\right) \\
& +g_{n}\left(\ln \left(\frac{t}{W}\right)\right)^{3}+h_{n}\left(\frac{a}{W}\right)^{3}+i_{n}\left(\frac{a}{W}\right)^{2} \ln \left(\frac{t}{W}\right) \\
& +j_{n}\left(\frac{a}{W}\right)\left(\ln \left(\frac{t}{W}\right)\right)^{2}
\end{aligned}
$$

where $\mathrm{n}=0,1,2,3$, and functions $F_{n}$ represents the normalized $K_{\mathrm{I}}$ along the crack front subjected to the uniform, linear, parabolic and cubic stress distribution, respectively. The 
parameters in the Eq. 3.7 are shown in the Table 3.30 for four different loading conditions. And the comparison of the prediction of stress intensity factor from finite element analysis and empirical equations are shown in Figure 3.33 (a)-(f). The maximum relative difference between the empirical formulae and the finite element results is less than $3 \%$.

\subsection{2 $T_{11}$ Equations}

The $T_{11}$ stress solutions at $z / t=0$ for stress applied on the crack front corresponding to the Eq. (3.1) to (3.4) are expressed in the following forms:

$$
T_{11}=\sigma_{0} V_{n}\left(\frac{a}{W}, \frac{t}{W}\right)
$$

where $\mathrm{n}=0,1,2,3$, and functions $V_{n}$ represents the normalized $T_{11}$ along the crack front subjected to the uniform, linear, parabolic and cubic stress field, respectively. For uniform stress field

$$
V_{0}=A_{0}+A_{1}\left(\frac{a}{W}\right)^{2}+A_{2}\left(\frac{a}{W}\right)^{4}+A_{3}\left(\frac{a}{W}\right)^{6}
$$

where

$$
\begin{aligned}
A_{0}=0.2026 & +0.01368\left(\frac{t}{W}\right)^{-1}+0.678\left(\frac{t}{W}\right)-0.9001\left(\frac{t}{W}\right)^{2} \\
& +0.4101\left(\frac{t}{W}\right)^{3}-0.05629\left(\frac{t}{W}\right)^{4} \\
A_{1}=6.021- & 0.1249\left(\frac{t}{W}\right)^{-1}-12.26\left(\frac{t}{W}\right)+14.66\left(\frac{t}{W}\right)^{2}-6.509\left(\frac{t}{W}\right)^{3} \\
& +0.8869\left(\frac{t}{W}\right)^{4} \\
A_{2}=-36.09+ & 0.9008\left(\frac{t}{W}\right)^{-1}+54.61\left(\frac{t}{W}\right)-55.95\left(\frac{t}{W}\right)^{2}+22.23\left(\frac{t}{W}\right)^{3} \\
& -2.842\left(\frac{t}{W}\right)^{4}
\end{aligned}
$$




$$
\begin{gathered}
A_{3}=75.2-1.047\left(\frac{t}{W}\right)^{-1}-57.8\left(\frac{t}{W}\right)+41.57\left(\frac{t}{W}\right)^{2}-12.09\left(\frac{t}{W}\right)^{3} \\
+1.222\left(\frac{t}{W}\right)^{4}
\end{gathered}
$$

The numerical results are approximated by empirical formulae fitted within an accuracy of $0.2 \%$.

For linear stress field

$$
V_{1}=B_{0}+B_{1}\left(\frac{a}{W}\right)^{2}+B_{2}\left(\frac{a}{W}\right)^{4}+B_{3}\left(\frac{a}{W}\right)^{6}
$$

where

$$
\begin{aligned}
B_{0}=0.1961+ & 0.003405\left(\frac{t}{W}\right)^{-1}+0.2411\left(\frac{t}{W}\right)-0.3853\left(\frac{t}{W}\right)^{2} \\
& +0.1882\left(\frac{t}{W}\right)^{3}-0.02664\left(\frac{t}{W}\right)^{4} \\
B_{1}=3.268- & 0.03388\left(\frac{t}{W}\right)^{-1}-4.291\left(\frac{t}{W}\right)+5.432\left(\frac{t}{W}\right)^{2}-2.535\left(\frac{t}{W}\right)^{3} \\
& +0.3551\left(\frac{t}{W}\right)^{4} \\
B_{2}=-22.25+ & 0.4137\left(\frac{t}{W}\right)^{-1}+23.32\left(\frac{t}{W}\right)-22.42\left(\frac{t}{W}\right)^{2}+8.624\left(\frac{t}{W}\right)^{3} \\
& -1.084\left(\frac{t}{W}\right)^{4} \\
B_{3}=47.99- & 0.5109\left(\frac{t}{W}\right)^{-1}-25.95\left(\frac{t}{W}\right)+13.93\left(\frac{t}{W}\right)^{2}-2.451\left(\frac{t}{W}\right)^{3} \\
& +0.09097\left(\frac{t}{W}\right)^{4}
\end{aligned}
$$

The most numerical results are approximated by empirical formulae fitted within an accuracy of $0.2 \%$, except $a / W=0.6, t / W=4$ is $3 \%$ and $a / W=0.8, t / W=4$ is $1.62 \%$.

For parabolic stress field

$$
V_{2}=C_{0}+C_{1}\left(\frac{a}{W}\right)^{2}+C_{2}\left(\frac{a}{W}\right)^{4}+C_{3}\left(\frac{a}{W}\right)^{6}
$$

where 


$$
\begin{aligned}
C_{0}=0.1505+ & 0.00004019\left(\frac{t}{W}\right)^{-1}+0.09923\left(\frac{t}{W}\right)-0.2013\left(\frac{t}{W}\right)^{2} \\
& +0.1056\left(\frac{t}{W}\right)^{3}-0.01539\left(\frac{t}{W}\right)^{4} \\
C_{1}=2.262+ & 0.01314\left(\frac{t}{W}\right)^{-1}-1.3\left(\frac{t}{W}\right)+1.957\left(\frac{t}{W}\right)^{2}-1.032\left(\frac{t}{W}\right)^{3} \\
& +0.1534\left(\frac{t}{W}\right)^{4} \\
C_{2}=-16.49+ & 0.1555\left(\frac{t}{W}\right)^{-1}+10.19\left(\frac{t}{W}\right)-8.77\left(\frac{t}{W}\right)^{2}+3.209\left(\frac{t}{W}\right)^{3} \\
& -0.3943\left(\frac{t}{W}\right)^{4}, \\
C_{3}=35.38- & 0.2299\left(\frac{t}{W}\right)^{-1}-12.37\left(\frac{t}{W}\right)+3.042\left(\frac{t}{W}\right)^{2}+1.04\left(\frac{t}{W}\right)^{3} \\
& -0.2923\left(\frac{t}{W}\right)^{4}
\end{aligned}
$$

The most numerical results are approximated by empirical formulae fitted within an accuracy of $0.7 \%$.

For cubic stress field

$$
V_{3}=D_{0}+D_{1}\left(\frac{a}{W}\right)^{2}+D_{2}\left(\frac{a}{W}\right)^{4}+D_{3}\left(\frac{a}{W}\right)^{6}
$$

where

$$
\begin{aligned}
D_{0}=0.105- & 0.00101\left(\frac{t}{W}\right)^{-1}+0.04351\left(\frac{t}{W}\right)-0.1168\left(\frac{t}{W}\right)^{2} \\
& +0.06545\left(\frac{t}{W}\right)^{3}-0.00978\left(\frac{t}{W}\right)^{4} \\
D_{1}=1.884+ & 0.03089\left(\frac{t}{W}\right)^{-1}-0.1371\left(\frac{t}{W}\right)+0.5278\left(\frac{t}{W}\right)^{2}-0.3939\left(\frac{t}{W}\right)^{3} \\
& +0.06635\left(\frac{t}{W}\right)^{4} \\
D_{2}=-13.71+ & 0.02488\left(\frac{t}{W}\right)^{-1}+4.163\left(\frac{t}{W}\right)-2.576\left(\frac{t}{W}\right)^{2}+0.787\left(\frac{t}{W}\right)^{3} \\
& -0.08874\left(\frac{t}{W}\right)^{4}
\end{aligned}
$$




$$
\begin{aligned}
D_{3}=28.3- & 0.07651\left(\frac{t}{W}\right)^{-1}-5.731\left(\frac{t}{W}\right)-1.867\left(\frac{t}{W}\right)^{2}+2.465\left(\frac{t}{W}\right)^{3} \\
& -0.4348\left(\frac{t}{W}\right)^{4}
\end{aligned}
$$

The most numerical results are approximated by empirical formulae fitted within an accuracy of $0.3 \%$.

The comparison of the prediction of $T_{11}$ stress from finite element analysis and empirical equations are shown in Figure 3.34 (a)-(f).

\subsection{3 $T_{33}$ Equations}

The $T_{33}$ solutions at $z / t=0$ for stress applied on the crack front corresponding to the Eq. (3.1) to (3.4) are expressed in the following forms:

$$
T_{33}=\sigma_{0} W_{n}\left(\frac{a}{W}, \frac{t}{W}\right)
$$

where $n=0,1,2,3$, and functions $W_{n}$ represents the normalized $T_{33}$ along the crack front subjected to the uniform, linear, parabolic and cubic stress field, respectively.

For uniform stress field

$$
W_{0}=A_{0}+A_{1}\left(\frac{a}{W}\right)^{2}+A_{2}\left(\frac{a}{W}\right)^{4}+A_{3}\left(\frac{a}{W}\right)^{6}
$$

where

$$
\begin{aligned}
A_{0}=0.1281 & -0.0267\left(\frac{t}{W}\right)^{-1}+0.01839\left(\frac{t}{W}\right)+0.09548\left(\frac{t}{W}\right)^{2} \\
& -0.08128\left(\frac{t}{W}\right)^{3}+0.014\left(\frac{t}{W}\right)^{4} \\
A_{1}=-8.526- & 0.7466\left(\frac{t}{W}\right)^{-1}+26.25\left(\frac{t}{W}\right)-28.57\left(\frac{t}{W}\right)^{2}+12.13\left(\frac{t}{W}\right)^{3} \\
& -1.621\left(\frac{t}{W}\right)^{4}
\end{aligned}
$$




$$
\begin{aligned}
A_{2}=22.45+ & 1.275\left(\frac{t}{W}\right)^{-1}-142.8\left(\frac{t}{W}\right)+182.9\left(\frac{t}{W}\right)^{2}-81.35\left(\frac{t}{W}\right)^{3} \\
& +11.03\left(\frac{t}{W}\right)^{4} \\
A_{3}=-59.84 & -3.621\left(\frac{t}{W}\right)^{-1}+320.9\left(\frac{t}{W}\right)-379.9\left(\frac{t}{W}\right)^{2}+162.4\left(\frac{t}{W}\right)^{3} \\
& -21.58\left(\frac{t}{W}\right)^{4}
\end{aligned}
$$

The numerical results are approximated by empirical formulae fitted within an accuracy of $2 \%$.

For linear stress field

$$
W_{1}=B_{0}+B_{1}\left(\frac{a}{W}\right)^{2}+B_{2}\left(\frac{a}{W}\right)^{4}+B_{3}\left(\frac{a}{W}\right)^{6}
$$

where

$$
\begin{aligned}
& B_{0}=-0.04633-0.01167\left(\frac{t}{W}\right)^{-1}+0.4873\left(\frac{t}{W}\right)-0.4911\left(\frac{t}{W}\right)^{2} \\
&+ 0.1835\left(\frac{t}{W}\right)^{3}-0.02235\left(\frac{t}{W}\right)^{4} \\
& B_{1}=-5.613- 0.3856\left(\frac{t}{W}\right)^{-1}+14\left(\frac{t}{W}\right)-13.47\left(\frac{t}{W}\right)^{2}+5.423\left(\frac{t}{W}\right)^{3} \\
&-0.7085\left(\frac{t}{W}\right)^{4} \\
& B_{2}=16.58+ 0.7248\left(\frac{t}{W}\right)^{-1}-87.39\left(\frac{t}{W}\right)+105.9\left(\frac{t}{W}\right)^{2}-46.07\left(\frac{t}{W}\right)^{3} \\
&+6.185\left(\frac{t}{W}\right)^{4} \\
& B_{3}=-40.44-2.294\left(\frac{t}{W}\right)^{-1}+202\left(\frac{t}{W}\right)-232.8\left(\frac{t}{W}\right)^{2}+98.31\left(\frac{t}{W}\right)^{3} \\
&-12.99\left(\frac{t}{W}\right)^{4}
\end{aligned}
$$

The numerical results are approximated by empirical formulae fitted within an accuracy of $2 \%$.

For parabolic stress field 


$$
W_{2}=C_{0}+C_{1}\left(\frac{a}{W}\right)^{2}+C_{2}\left(\frac{a}{W}\right)^{4}+C_{3}\left(\frac{a}{W}\right)^{6}
$$

where

$$
\begin{aligned}
C_{0}=-0.07497- & 0.0026\left(\frac{t}{W}\right)^{-1}+0.5095\left(\frac{t}{W}\right)-0.5498\left(\frac{t}{W}\right)^{2} \\
+ & 0.2181\left(\frac{t}{W}\right)^{3}-0.02765\left(\frac{t}{W}\right)^{4} \\
C_{1}=-3.994- & 0.3259\left(\frac{t}{W}\right)^{-1}+8.371\left(\frac{t}{W}\right)-6.862\left(\frac{t}{W}\right)^{2}+2.544\left(\frac{t}{W}\right)^{3} \\
& -0.3202\left(\frac{t}{W}\right)^{4} \\
C_{2}=13.78+ & 0.8233\left(\frac{t}{W}\right)^{-1}-61.2\left(\frac{t}{W}\right)+69.47\left(\frac{t}{W}\right)^{2}-29.43\left(\frac{t}{W}\right)^{3} \\
& +3.905\left(\frac{t}{W}\right)^{4} \\
C_{3}=-31.52- & 1.984\left(\frac{t}{W}\right)^{-1}+144.8\left(\frac{t}{W}\right)-161.6\left(\frac{t}{W}\right)^{2}+67.31\left(\frac{t}{W}\right)^{3} \\
& -8.834\left(\frac{t}{W}\right)^{4}
\end{aligned}
$$

The most numerical results are approximated by empirical formulae fitted with an accuracy of $5 \%$, except $t / W=4.0$ is about $10 \%$.

For cubic stress field

$$
W_{3}=D_{0}+D_{1}\left(\frac{a}{W}\right)^{2}+D_{2}\left(\frac{a}{W}\right)^{4}+D_{3}\left(\frac{a}{W}\right)^{6}
$$

Where

$$
\begin{aligned}
D_{0}=0.01499 & -0.2554\left(\frac{t}{W}\right)^{0.5}+0.6875\left(\frac{t}{W}\right)-0.5958\left(\frac{t}{W}\right)^{2} \\
& +0.2332\left(\frac{t}{W}\right)^{3}-0.02975\left(\frac{t}{W}\right)^{4} \\
D_{1}=-15.6+ & 43.73\left(\frac{t}{W}\right)^{0.5}-42.05\left(\frac{t}{W}\right)+18.84\left(\frac{t}{W}\right)^{2}-6.124\left(\frac{t}{W}\right)^{3} \\
& +0.7315\left(\frac{t}{W}\right)^{4}
\end{aligned}
$$




$$
\begin{aligned}
D_{2}=51.76- & 137.7\left(\frac{t}{W}\right)^{0.5}+103.2\left(\frac{t}{W}\right)-21.97\left(\frac{t}{W}\right)^{2}+3.123\left(\frac{t}{W}\right)^{3} \\
& -0.1584\left(\frac{t}{W}\right)^{4} \\
D_{3}=-102.8+ & 269.1\left(\frac{t}{W}\right)^{0.5}-183.9\left(\frac{t}{W}\right)+21.17\left(\frac{t}{W}\right)^{2}+3.131\left(\frac{t}{W}\right)^{3} \\
- & 0.9084\left(\frac{t}{W}\right)^{4}
\end{aligned}
$$

The most numerical results are approximated by empirical formulae fitted within an accuracy of $3 \%$.

The comparison of the prediction of $T_{33}$ stress from finite element analysis and empirical equations are shown in Figure 3.35 (a)-(f).

Using the superposition method discussed in Chapter 2, these empirical equations can be used to calculate $K_{l}, T_{11}$ and $T_{33}$ for various linear and nonlinear stress distributions.

\subsection{SUMMARY}

In this chapter, the finite element model of the three-dimensional single edge cracked plate specimens with different relative crack depth and thickness were built in ABAQUS (2008). The correspondence stress intensity factor $K_{I}$ and $T$-stresses $\left(T_{11}\right.$ and $T_{33}$ ) for the crack surface subjected to constant, linear, parabolic or cubic stress distribution were obtained. And the approximated empirical formulae were fitted for the numerical stress intensity factor and $T$-stresses results for cracks at position $z / t=0$.

In next chapter, the influence of the crack depth, plate thickness and the variation of $K_{I}, T_{11}$ and $T_{33}$ along the thickness are discussed, respectively. 
Table 3.1 Comparison of the $\mathrm{K}$ from present FEM calculation and solution form $\mathrm{Qu}$ and Wang for surface corner crack, $a / c=1.0, a / t=0.2$

\begin{tabular}{|c|c|c|c|}
\hline Location $\phi^{0}$ & $\begin{array}{c}\text { Normalized } K \\
\text { (Qu and Wang, 2006) }\end{array}$ & $\begin{array}{c}\text { Normalized } K \\
\text { Present FEM }\end{array}$ & Difference (\%) \\
\hline 0.00 & 1.1763 & 1.1382 & 3.24 \\
\hline 90.00 & 1.1094 & 1.0828 & 2.39 \\
\hline
\end{tabular}

Table 3.2 Comparison of the $T_{11}$ from present FEM calculation and solution form Qu and Wang for surface corner crack, $a / c=1.0, a / t=0.2$

\begin{tabular}{|c|c|c|c|}
\hline Location $\phi^{0}$ & $\begin{array}{c}\text { Normalized } T_{11} \\
(\mathrm{Qu} \text { and Wang, 2006) }\end{array}$ & $\begin{array}{c}\text { Normalized } T_{11} \\
\text { Present FEM }\end{array}$ & Difference (\%) \\
\hline 5.00 & -0.4370 & -0.4506 & 3.12 \\
\hline 7.50 & -0.4229 & -0.4155 & 1.76 \\
\hline 10.00 & -0.4113 & -0.4018 & 2.31 \\
\hline 12.50 & -0.4027 & -0.3946 & 2.01 \\
\hline 15.00 & -0.3967 & -0.3900 & 1.70 \\
\hline 17.50 & -0.3926 & -0.3867 & 1.51 \\
\hline 20.00 & -0.3885 & -0.3842 & 1.11 \\
\hline 22.50 & -0.3824 & -0.3823 & 0.02 \\
\hline 30.00 & -0.3819 & -0.3789 & 0.79 \\
\hline 37.50 & -0.3798 & -0.3774 & 0.64 \\
\hline 45.00 & -0.3790 & -0.3770 & 0.52 \\
\hline 52.50 & -0.3791 & -0.3775 & 0.41 \\
\hline 60.00 & -0.3799 & -0.3793 & 0.16 \\
\hline 67.50 & -0.3834 & -0.3831 & 0.09 \\
\hline 75.00 & -0.3879 & -0.3909 & 0.78 \\
\hline 82.50 & -0.4269 & -0.4169 & 2.33 \\
\hline 86.25 & -0.4727 & -0.4616 & 2.34 \\
\hline
\end{tabular}


Table 3.3 Comparison of the $T_{33}$ from present FEM calculation and solution form Qu and Wang for surface corner crack, $a / c=1.0, a / t=0.2$

\begin{tabular}{|c|c|c|c|}
\hline Location $\phi^{0}$ & $\begin{array}{c}\text { Normalized } T_{33} \\
(\mathrm{Qu} \text { and Wang, 2006) }\end{array}$ & $\begin{array}{c}\text { Normalized } T_{33} \\
\text { Present FEM }\end{array}$ & Difference (\%) \\
\hline 86.25 & -1.3264 & -1.23129 & 7.17 \\
\hline 82.50 & -0.8885 & -0.93139 & 4.83 \\
\hline 75.00 & -0.8191 & -0.81491 & 0.51 \\
\hline 67.50 & -0.7944 & -0.77874 & 1.97 \\
\hline 60.00 & -0.783 & -0.76756 & 1.97 \\
\hline 52.50 & -0.7798 & -0.76434 & 1.98 \\
\hline 45.00 & -0.7793 & -0.76381 & 1.99 \\
\hline 37.50 & -0.7796 & -0.76481 & 1.90 \\
\hline 30.00 & -0.7817 & -0.76852 & 1.69 \\
\hline 22.50 & -0.7829 & -0.78016 & 0.35 \\
\hline 20.00 & -0.7825 & -0.78801 & 0.70 \\
\hline 17.50 & -0.7921 & -0.79961 & 0.95 \\
\hline 15.00 & -0.8057 & -0.81677 & 1.37 \\
\hline 12.50 & -0.8255 & -0.84221 & 2.02 \\
\hline 10.00 & -0.8563 & -0.87992 & 2.76 \\
\hline 7.50 & -0.9004 & -0.93387 & 3.72 \\
\hline 5.00 & -0.9737 & -1.00256 & 2.96 \\
\hline
\end{tabular}

Table 3.4 Comparison of $T_{11}$ from present FEM calculation and solution from Nakamura and Parks (1992) for $\mathrm{a} / \mathrm{W}=0.5, \mathrm{t} / \mathrm{W}=0.5$

\begin{tabular}{|c|c|c|c|c|c|c|c|c|c|}
\hline \multirow{2}{*}{$z / t$} & \multicolumn{3}{|c|}{$v=0$} & \multicolumn{3}{c|}{$v=0.3$} & \multicolumn{3}{c|}{$v=0.499$} \\
\cline { 2 - 10 } & Present & $\begin{array}{c}\text { Nakamura } \\
\text { and Parks } \\
(1992)\end{array}$ & $\%$ & Present & $\begin{array}{c}\text { Nakamura } \\
\text { and Parks } \\
(1992)\end{array}$ & $\%$ & Present & $\begin{array}{c}\text { Nakamura } \\
\text { and Parks } \\
(1992)\end{array}$ & $\%$ \\
\hline 0.00 & -0.4124 & -0.4364 & 5.50 & -0.2079 & -0.2105 & 1.24 & 0.0983 & 0.1006 & 2.29 \\
\hline 0.25 & -0.4124 & -0.4364 & 5.50 & -0.2138 & -0.2289 & 6.60 & 0.1111 & 0.1135 & 2.11 \\
\hline 0.40 & -0.4124 & -0.4364 & 5.50 & -0.2232 & -0.2432 & 8.22 & 0.1724 & 0.1973 & 12.6 \\
\hline
\end{tabular}


Table 3.5 Comparison of stress intensity factor and $T_{11}$ from present FEM calculation and solution from Sham (1991) for $a / W=0.5$

\begin{tabular}{|c|c|c|c|c|c|c|}
\hline & $K_{1} / \sigma \sqrt{\pi a}$ & $\begin{array}{c}\text { Difference } \\
(\%) \\
100 \times \\
\left|F_{p}-F_{s}\right| / F_{s}\end{array}$ & $\begin{array}{l}\beta \\
\equiv T_{11} \sqrt{\pi a} / K_{I}\end{array}$ & $\begin{array}{c}\text { Difference } \\
(\%) \\
100 \times \\
\left|F_{p}-F_{s}\right| / F_{s}\end{array}$ & $T_{11} / \sigma$ & $\begin{array}{c}\text { Difference } \\
(\%) \\
100 \times \\
\left|F_{p}-F_{s}\right| / F_{s}\end{array}$ \\
\hline $\begin{array}{c}\text { Present } \\
\text { calculation } \\
\left(F_{p}\right)\end{array}$ & 2.824 & \multirow{2}{*}{0.11} & -0.15301 & \multirow{2}{*}{0.05} & -0.42314 & \multirow{2}{*}{1.91} \\
\hline $\begin{array}{c}\text { Sham's } \\
\text { result }\left(F_{s}\right) \\
(1991)\end{array}$ & 2.821 & & -0.15293 & & -0.43142 & \\
\hline
\end{tabular}


Table 3.6 Normalized $K_{1}, T_{11}$ and $T_{33}$ for $\mathrm{a} / \mathrm{W}=0.2, \mathrm{t} / \mathrm{W}=0.1$, SECP

\begin{tabular}{|c|c|c|c|c|c|c|c|c|c|c|c|c|}
\hline \multirow{2}{*}{$2 / t$} & \multicolumn{3}{|c|}{ Uniform } & \multicolumn{3}{|c|}{ Linear } & \multicolumn{3}{|c|}{ Parabolic } & \multicolumn{3}{|c|}{ Cubic } \\
\hline & $K_{I}$ & $T_{l 1}$ & $T_{33}$ & $K_{I}$ & $T_{l l}$ & $T_{33}$ & $K_{I}$ & $T T_{I I}$ & $T_{33}$ & $K_{I}$ & $\bar{T}$ & $T_{33}$ \\
\hline 0.0000 & 1.4535 & 0.5144 & -0.6499 & 0.6307 & 0.3297 & -0.4241 & 0.4022 & 0.2298 & -0.2906 & 0.2816 & 0.1664 & -0.2076 \\
\hline 0.0346 & 1.4534 & 0.5144 & -0.6533 & 0.6305 & 0.3295 & -0.4258 & 0.4021 & 0.2297 & -0.2916 & 0.2816 & 0.1663 & -0.2083 \\
\hline 0.0679 & 1.4530 & 0.5152 & -0.6628 & 0.6302 & 0.3297 & -0.4306 & 0.4019 & 0.2297 & -0.2945 & 0.2814 & 0.1663 & -0.2103 \\
\hline 0.1001 & 1.4524 & 0.5165 & -0.6783 & 0.6296 & 0.3298 & -0.4384 & 0.4015 & 0.2298 & -0.2993 & 0.2811 & 1664 & -0.2135 \\
\hline 0.1311 & 1.4516 & 0.5182 & -0.6997 & 0.6289 & 0.3301 & -0.4490 & 0.4009 & 0.2298 & 057 & 0.2807 & 0.1664 & 179 \\
\hline 0.1610 & 1.4505 & 0.5203 & -0.7269 & 0.6279 & 0.3303 & -0.4624 & 0.4002 & 0.2299 & -0.3139 & 0.2802 & 0.1665 & -0.2235 \\
\hline 0.1898 & 1.4492 & 0.5228 & -0.7604 & 0.6267 & 0.3307 & -0.4788 & 0.3994 & 0.2301 & -0.3239 & 0.2796 & 0.1666 & -0.2304 \\
\hline 0.2176 & 1.4475 & 0.5256 & -0.8006 & 53 & 11 & -0.4 & 0.3984 & 303 & 59 & 0.2789 & 1668 & 385 \\
\hline 0.2444 & 1.4455 & 0.5288 & -0.8484 & 0.6237 & 0.3315 & -0.5214 & 0.3972 & 0.2305 & -0.3499 & 0.2781 & 0.1670 & -0.2482 \\
\hline 0.2702 & 1.4431 & 0.5324 & -0.9047 & 0.6217 & 0.3320 & -0.5484 & 0.3959 & 0.2308 & -0.3664 & 0.2771 & 0.1672 & -0.2595 \\
\hline 0.2951 & 1.4402 & 0.5362 & -0.9713 & 0.6195 & 0.3325 & -0.5798 & 0.3943 & 0.2312 & -0.3857 & 0.2760 & 0.1676 & -0.2728 \\
\hline 0.3191 & 1.4368 & 0.5403 & -1.0502 & 0.6169 & 0.3331 & -0.6167 & 0.3926 & 0.2316 & -0.4083 & 0.2747 & 0.1680 & -0.2884 \\
\hline 0.3423 & 1.4327 & 0.5447 & -1.1448 & 0.6138 & 0.3337 & -0.6603 & 0.3905 & 0.2322 & -0.4351 & 0.2733 & 0.1685 & -0.3069 \\
\hline 0.3646 & 1.4276 & 0.5492 & -1.2599 & 0.6103 & 0.3343 & -0.7128 & 0.3881 & 0.2328 & -0.4675 & 0.2716 & 0.1691 & -0.3293 \\
\hline 0.3861 & 1.4214 & 0.5539 & -1.4035 & 0.6060 & 0.3349 & -0.7773 & 0.3853 & 0.2336 & -0.5074 & 0.2696 & 0.1698 & -0.3570 \\
\hline 0.4069 & 1.4136 & 0.5585 & -1.5888 & 0.6009 & 0.3355 & -0.8594 & 0.3819 & 0.2344 & -0.5583 & 0.2673 & 0.1707 & -0.3924 \\
\hline 0.4269 & 1.4035 & 0.5637 & -1.8433 & 0.5945 & 0.3363 & -0.9705 & 0.3778 & 0.2356 & -0.6276 & 0.2644 & 0.1717 & -0.4406 \\
\hline 0.4462 & 1.3899 & 0.5653 & -2.2089 & 0.5864 & 0.3356 & -1.1279 & 0.3726 & 0.2362 & -0.7261 & 0.2608 & 0.1724 & -0.5094 \\
\hline 0.4648 & 1.3716 & 0.5499 & -2.7787 & 0.5759 & 0.3281 & -1.3705 & 0.3659 & 0.2327 & -0.8785 & 0.2561 & 0.1703 & -0.6159 \\
\hline 0.4827 & 1.3423 & 0.2544 & -3.2018 & 0.5600 & 0.2055 & -1.5530 & 0.3559 & 0.1564 & -0.9924 & 0.2492 & 0.1172 & -0.6955 \\
\hline 0.5000 & 1.1833 & 0.1095 & -11.4792 & 0.4881 & 0.1594 & -4.9707 & 0.3105 & 0.1292 & -3.1642 & 0.2175 & 0.0982 & -2.2165 \\
\hline
\end{tabular}


Table 3.7 Normalized $K_{\mathrm{l}}, T_{11}$ and $T_{33}$ for $\mathrm{a} / \mathrm{W}=0.2, \mathrm{t} / \mathrm{W}=0.2$, SECP

\begin{tabular}{|c|c|c|c|c|c|c|c|c|c|c|c|c|}
\hline \multirow{2}{*}{$z / t$} & \multicolumn{3}{|c|}{ Uniform } & \multicolumn{3}{|c|}{ Linear } & \multicolumn{3}{|c|}{ Parabolic } & \multicolumn{3}{|c|}{ Cubic } \\
\hline & $K_{I}$ & $T_{I I}$ & $T_{33}$ & $K_{I}$ & $T_{\mu}$ & $T_{33}$ & $K_{I}$ & $T_{l l}$ & $T_{33}$ & $K_{I}$ & $T_{l l}$ & $T_{33}$ \\
\hline 000 & 4493 & & & 334 & & & 4053 & & & 2842 & & \\
\hline & & & & & & & & & & & & \\
\hline & & & & & & & 15 & & & 2836 & & \\
\hline 0.136 & 447 & & & 12 & & & 4036 & 75 & & 2829 & & \\
\hline 0.1747 & 4457 & & -0. & 97 & 6 & -0.2 & \begin{tabular}{|l|}
0.4024 \\
\end{tabular} & 2268 & -0.1867 & 0.2820 & & \\
\hline 0.2098 & 1.4438 & 0. & -0.4 & 6278 & 3 & -0.2 & 0.4010 & 2260 & -0.1986 & 0.2809 & 45 & 438 \\
\hline 0.2424 & 4416 & 0.4 & & 56 & 8 & -0.3 & 0.3993 & 51 & & 0.2797 & & \\
\hline 0.2722 & 4388 & 0. & -0.4 & 30 & 0 & & 0.3973 & & -0 & 0.2782 & & \\
\hline 0.2998 & 57 & & & 01 & & & 50 & 30 & 51 & 2765 & & \\
\hline 250 & 1320 & & & 167 & & & 25 & 9 & 47 & 2746 & & \\
\hline 484 & 278 & & & 29 & & & 3897 & 07 & 65 & 2726 & & \\
\hline 3698 & 228 & & & 5086 & & & 0.3866 & 98 & 13 & .2703 & & \\
\hline 3896 & 170 & & & 5038 & & & 3831 & 86 & 94 & 0.2678 & & \\
\hline 4076 & 1 & & & & & & 0. & & & 0.2650 & & \\
\hline & 1.4021 & & & & & & 0.3 & & & 0.2620 & & \\
\hline 0.4397 & 3922 & & & 850 & & -0.7 & \begin{tabular}{|l|}
0.3701 \\
\end{tabular} & 54 & -0 & 0.2585 & & \\
\hline 0.4539 & 1.3800 & 0.5213 & -1.5 & 0.5767 & 0.3082 & -0.8096 & \begin{tabular}{|l|}
0.3645 \\
\end{tabular} & \begin{tabular}{|l|}
0.2153 \\
\end{tabular} & \begin{tabular}{|l}
-0.5229 \\
\end{tabular} & 0.2545 & & -0.3666 \\
\hline 0.4668 & 1.3636 & 0. & & 0.5662 & 3072 & -0.9562 & 0.3575 & 0.2157 & -0.6134 & 0.2496 & 1576 & 0.4294 \\
\hline 0.4789 & 1.3412 & 0. & -2.4 & 0.5528 & 3005 & -1.1860 & \begin{tabular}{|l|l|l}
0.3488 \\
\end{tabular} & 0.2129 & -0.7561 & 0.2435 & 1561 & -0.5287 \\
\hline 4898 & 968 & & & 995 & 55 & & 0.3340 & 0.2121 & -1.0368 & 2331 & .1560 & -0.7243 \\
\hline & & .08 & $11.1 / 34$ & 4547 & .0779 & -4.9612 & 0.2869 & 0.0781 & -3.1319 & 2003 & .0629 & -2.186 \\
\hline
\end{tabular}

45 
Table 3.8 Normalized $K_{1}, T_{11}$ and $T_{33}$ for $\mathrm{a} / \mathrm{W}=0.2, \mathrm{t} / \mathrm{W}=0.5$, SECP

\begin{tabular}{|c|c|c|c|c|c|c|c|c|c|c|c|c|}
\hline \multirow{2}{*}{$z / t$} & \multicolumn{3}{|c|}{ Uniform } & \multicolumn{3}{|c|}{ Linear } & \multicolumn{3}{|c|}{ Parabolic } & \multicolumn{3}{|c|}{ Cubic } \\
\hline & $K_{I}$ & $T_{\|}$ & $T_{33}$ & $K_{I}$ & $T_{\| I}$ & $T_{33}$ & $K_{I}$ & $T_{l l}$ & $T_{33}$ & $K_{I}$ & $T_{11}$ & $T_{33}$ \\
\hline 0.0000 & 1.4346 & 0.4676 & -0.0215 & 0.6281 & 0.3131 & -0.0187 & 0.4028 & 0.2237 & -0.0142 & 0.2827 & 0.1639 & -0.0107 \\
\hline 0.0615 & 1.4343 & 0.4669 & -0.0260 & 0.6278 & 0.3127 & -0.0221 & 0.4026 & 0.2234 & -0.0167 & 0.2826 & 0.1637 & -0.0125 \\
\hline 0.1161 & 1.4334 & 0.4652 & -0.0379 & 0.6271 & 0.3117 & -0.0309 & 0.4020 & 0.2227 & -0.0232 & 0.2822 & 0.1632 & -0.0173 \\
\hline 0.1647 & 1.4320 & 0.4628 & -0.0556 & 6259 & 0.3102 & -0.0441 & 0.4011 & 0.2217 & -0.0328 & 0.2815 & 1624 & -0.0243 \\
\hline 0.2078 & 1.4301 & 01 & 84 & 44 & 83 & 08 & 0.3999 & 02 & 50 & 06 & 613 & 333 \\
\hline 0.2462 & 1.4277 & 0.4572 & -0 . & 0.6223 & 0.3061 & -0.0808 & 0.3983 & 0.2184 & -0.0594 & 0.2794 & 599 & -0.0438 \\
\hline 0.2802 & 1.4247 & 45 & 84 & 98 & 036 & 38 & 964 & 163 & -0.0757 & 0.2779 & 582 & -0.0557 \\
\hline 0.3105 & 1.4212 & 21 & -0. & 67 & 008 & -0.1296 & 940 & 0.2139 & -0.0938 & 0.2761 & 0.1563 & -0.0688 \\
\hline 0.3374 & 1.4171 & 0.4502 & -0.2185 & 0.6130 & 0.2979 & -0.1582 & 0.3912 & 0.2111 & -0.1136 & 0.2740 & 0.1540 & -0.0829 \\
\hline 0.3613 & 1.4123 & 0.4489 & -0.2675 & 0.6088 & 0.2946 & -0.1898 & 0.3879 & 0.2081 & -0.1349 & 0.2716 & 0.1515 & -0.0980 \\
\hline 0.3825 & 1.4069 & 0.4482 & -0.3239 & 0.6040 & 0.2912 & -0.2245 & 0.3842 & 0.2047 & -0.1578 & 0.2687 & 0.1488 & -0.1141 \\
\hline 0.4014 & 1.4007 & 0.4480 & -0.3894 & 0.5985 & 0.2874 & -0.2628 & 0.3800 & 0.2010 & -0.1826 & 0.2656 & 0.1458 & -0.1313 \\
\hline 0.4182 & 1.3937 & 0.4483 & -0.4662 & 0.5922 & 0.2834 & -0.3056 & 0.3753 & 0.1972 & -0.2096 & 0.2621 & 0.1428 & -0.1499 \\
\hline 0.4331 & 1.3857 & 0.4491 & -0.5579 & 0.5852 & 0.2790 & -0.3539 & 0.3700 & 0.1932 & -0.2396 & 0.2582 & 0.1397 & -0.1704 \\
\hline 0.4464 & 1.3763 & 0.4500 & -0.6699 & 0.5773 & 0.2744 & -0.4099 & 0.3642 & 0.1892 & -0.2738 & 0.2539 & 0.1367 & -0.1938 \\
\hline 0.4581 & 1.3653 & 0.4509 & -0.8112 & 0.5683 & 0.2694 & -0.4771 & 0.3577 & 0.1853 & -0.3145 & 0.2491 & 0.1339 & -0.2215 \\
\hline 0.4686 & 1.3519 & 0.4516 & -0.9981 & 0.5578 & 0.2643 & -0.5617 & 0.3503 & 0.1817 & -0.3655 & 0.2438 & 0.1315 & -0.2563 \\
\hline 0.4779 & 1.3347 & 0.4511 & -1.2648 & 0.5454 & 0.2586 & -0.6773 & 0.3417 & 0.1784 & -0.4352 & 0.2376 & 0.1295 & -0.3041 \\
\hline 0.4861 & 1.3110 & 0.4489 & -1.7038 & 0.5295 & 0.2527 & -0.8599 & 0.3310 & 0.1757 & -0.5461 & 0.2301 & 0.1280 & -0.3805 \\
\hline 0.4935 & 1.2737 & 0.4269 & -2.6392 & 0.5071 & 0.2402 & -1.2365 & 0.3164 & 0.1703 & -0.7772 & 0.2198 & 0.1249 & -0.5404 \\
\hline 0.5000 & 1.0900 & -0.0266 & -10.7843 & 0.4230 & 0.0825 & -4.4048 & 0.2636 & 0.0774 & -2.7464 & 0.1831 & 0.0614 & -1.9073 \\
\hline
\end{tabular}

46 
Table 3.9 Normalized $K_{1}, T_{11}$ and $T_{33}$ for $\mathrm{a} / \mathrm{W}=0.2, \mathrm{t} / \mathrm{W}=1.0, \mathrm{SECP}$

\begin{tabular}{|c|c|c|c|c|c|c|c|c|c|c|c|c|}
\hline \multirow{2}{*}{$z / t$} & \multicolumn{3}{|c|}{ Uniform } & \multicolumn{3}{|c|}{ Linear } & \multicolumn{3}{|c|}{ Parabolic } & \multicolumn{3}{|c|}{ Cubic } \\
\hline & $K_{I}$ & $T_{11}$ & $T_{33}$ & $K_{I}$ & $T_{I I}$ & 33 & $K_{I}$ & $T_{I I}$ & 133 & $\boldsymbol{K}_{I}$ & $T_{l l}$ & $T_{33}$ \\
\hline 000 & 1057 & & & 097 & & & 8899 & & & 2734 & & \\
\hline 515 & 059 & & & 099 & & & 900 & & 41 & & & \\
\hline 0.116 & 4065 & J & 61 & 6102 & 0.2877 & 0.0511 & 0.3903 & 10 & .0407 & 2737 & 99 & \\
\hline 0.1647 & \begin{tabular}{|l|l|}
1.4071 \\
\end{tabular} & 0 & 56 & 0.6106 & 0.2898 & 0.0497 & 0.3906 & 2065 & 0.0354 & .2739 & & .0259 \\
\hline 0.2078 & 1.4075 & $43\}$ & 0.0616 & 0.6109 & 0.2919 & 0.0398 & \begin{tabular}{|l|}
0.3908 \\
\end{tabular} & \begin{tabular}{|l|}
0.2081 \\
\end{tabular} & 0.0282 & 0.2741 & 1523 & 0.0206 \\
\hline 0.2462 & 1.4076 & 0.4404 & 0.0442 & 0.6109 & 0.2935 & 0.0272 & \begin{tabular}{|l|}
0.3908 \\
\end{tabular} & 0.2094 & 0.0190 & 0.2741 & & 0.0138 \\
\hline 0.2802 & 1.4072 & 0.44 & 0.02 & 0.6106 & \begin{tabular}{|l|l|}
0.2945 \\
\end{tabular} & 0.0120 & 0.3905 & & 0.0079 & 0.2738 & & \\
\hline 0.3105 & 4062 & 0.4 & -0.0 & 0.6097 & \begin{tabular}{|l|l|}
0.2 \\
\end{tabular} & -0.0 & 0.3898 & & -0.6 & & & \\
\hline 0.3374 & 5 & & & & & -0 & 88 & & 04 & & & \\
\hline 0.3613 & 1 & & & 63 & 0 & -0 & 72 & & 76 & & & \\
\hline 0.3825 & 3988 & & -0 & 35 & 0 & -0.07 & 0.3851 & 2069 & $\mid-0.0570$ & 998 & & \\
\hline 0.4014 & 3946 & & 435 & 5999 & 12 & -0.1 & 3823 & 2044 & $\begin{array}{c}-0.0788 \\
\end{array}$ & 2677 & & \\
\hline 0.4182 & 3895 & & -0.1954 & 5954 & 88 & -0.1 & \begin{tabular}{|l|}
0.3789 \\
\end{tabular} & 3 & \begin{tabular}{|l|}
-0.1030 \\
\end{tabular} & 51 & & \\
\hline 0.433 & 83 & & & 9 & 8 & -0.1 & \begin{tabular}{|l|}
0.3746 \\
\end{tabular} & & $\begin{array}{l}-0.1300 \\
\end{array}$ & & & \\
\hline 0.4464 & 3756 & & -0.3347 & 0.5831 & 0.2 & -0.2 & 0.3694 & & -0.1604 & & & \\
\hline 0.4581 & 1.3662 & & -0.4327 & 0.5748 & 0.2697 & -0.2848 & 0.3631 & 0.1 & -0.1954 & 33 & & -0. \\
\hline 0.4686 & 1.3546 & 0.4270 & -0.5642 & 0.5647 & 0.2635 & -0.3532 & \begin{tabular}{|l|}
0.3555 \\
\end{tabular} & 0.1822 & -0.2377 & 0.2476 & 0.1317 & -0. \\
\hline 0.4779 & \begin{tabular}{|l}
1.3393 \\
\end{tabular} & 0.4284 & -0.7547 & 0.5520 & 0.2563 & -0.4446 & \begin{tabular}{|l|}
0.3462 \\
\end{tabular} & 0.1763 & -0.2930 & \begin{tabular}{|l|}
0.2408 \\
\end{tabular} & 0.1273 & -0.2062 \\
\hline 0.4861 & 1.3178 & & -1.0621 & 0.5352 & 0.2475 & -0.5816 & 0.3344 & 0.1702 & -0.3752 & 0.2323 & 0.1233 & -0.2623 \\
\hline 0.4935 & 1.2824 & & -1.5826 & 0.5106 & 0.2189 & -0.8018 & \begin{tabular}{|l|}
0.3177 \\
\end{tabular} & 0.1538 & \begin{tabular}{|l|}
-0.5071 \\
\end{tabular} & \begin{tabular}{|l}
0.2204 \\
\end{tabular} & \begin{tabular}{|l}
0.1126 \\
\end{tabular} & -0.3527 \\
\hline 0.5000 & 1031 & 0.2144 & 8.8316 & 4226 & .1634 & 3.594 & 2619 & 0.1279 & .2 & 0.1815 & 0962 & -1.546 \\
\hline
\end{tabular}


Table 3.10 Normalized $K_{1}, T_{11}$ and $T_{33}$ for $\mathrm{a} / \mathrm{W}=0.2, \mathrm{t} / \mathrm{W}=2.0, \mathrm{SECP}$

\begin{tabular}{|c|c|c|c|c|c|c|c|c|c|c|c|c|}
\hline \multirow{2}{*}{$2 / t$} & \multicolumn{3}{|c|}{ Uniform } & \multicolumn{3}{|c|}{ Linear } & \multicolumn{3}{|c|}{ Parabolic } & \multicolumn{3}{|c|}{ Cubic } \\
\hline & $\bar{K}$ & $T_{l l}$ & $T_{33}$ & $K_{I}$ & $T_{11}$ & $T_{33}$ & $K_{l}$ & $T_{l l}$ & $T_{33}$ & $K_{I}$ & $T_{n}$ & $T_{33}$ \\
\hline 0.0000 & 1.3763 & 0.4079 & 0.1169 & 0.5912 & 0.2709 & 0.0778 & 0.3770 & 0.1931 & 0.0555 & 0.2641 & 0.1413 & 0.0406 \\
\hline 0.0615 & 1.3768 & 0.4084 & 0.1167 & 0.5915 & 0.2712 & 0.0777 & 0.3773 & 0.1933 & 0.0554 & 0.2643 & 0.1415 & 0.0406 \\
\hline 0.1161 & 1.3782 & 0.4096 & 0.1159 & 0.5924 & 0.2720 & 0.0772 & 0.3778 & 0.1938 & 0.0551 & 0.2647 & 0.1419 & 0.0403 \\
\hline 0.1647 & 1.3801 & 0.4118 & 0.1144 & 0.5936 & 0.2733 & 0.0762 & 0.3787 & 0.1948 & 0.0543 & 0.2653 & 0.1425 & 0.0398 \\
\hline 0.2078 & 1.3825 & 0. & 0.1 & 0.5951 & 0.2753 & 0.0744 & 0.3798 & 0.1961 & 0. & 0.2661 & 435 & 0.0389 \\
\hline 0.2462 & 1.3851 & 0.4187 & 0.1073 & 0.5968 & 0.2778 & 0.0714 & 0.3809 & 0.1979 & 0.0509 & 0.2669 & 0.1448 & 0.0373 \\
\hline 0.2802 & 1.3877 & 234 & 0 . & 0.5985 & 809 & 0.0667 & 0.3821 & 0.2001 & 0.0476 & 0.2678 & 0.1464 & 0.0349 \\
\hline 0.3105 & 1.3901 & 283 & 0 & 0.6000 & 0.2844 & 0.0598 & 0.3832 & 0.2026 & 0.0427 & 0.2686 & 0.1483 & 0.0312 \\
\hline 0.3374 & 1.3921 & 0.4331 & 0.0766 & 0.6013 & 0.2879 & 0.0503 & 0.3841 & 0.2052 & 0.0357 & 0.2692 & 0.1502 & 0.0261 \\
\hline 0.3613 & 1.3934 & 0.4368 & 0.0587 & 0.6021 & 0.2909 & 0.0375 & 0.3846 & 0.2075 & 0.0265 & 0.2696 & 0.1519 & 0.0193 \\
\hline 0.3825 & 1.3938 & 0.4389 & 0.0362 & 0.6023 & 0.2930 & 0.0213 & 0.3848 & 0.2092 & 0.0146 & 0.2697 & 0.1532 & 0.0105 \\
\hline 0.4014 & 1.3933 & 0.4392 & 0.0086 & 0.6018 & 0.2939 & 0.0012 & 0.3843 & 0.2100 & -0.0001 & 0.2694 & 0.1538 & -0.0004 \\
\hline 0.4182 & 1.3916 & 0.4377 & -0.0251 & 0.6003 & 0.2934 & -0.0236 & 0.3832 & 0.2097 & -0.0182 & 0.2685 & 0.1536 & -0.0137 \\
\hline 0.4331 & 1.3887 & 0.4349 & -0.0665 & 0.5978 & 0.2916 & -0.0537 & 0.3813 & 0.2083 & -0.0400 & 0.2671 & 0.1525 & -0.0297 \\
\hline 0.4464 & 1.3842 & 0.4316 & -0.1183 & 0.5939 & 0.2886 & -0.0905 & 0.3783 & 0.2057 & -0.0663 & 0.2648 & 0.1505 & -0.0488 \\
\hline 0.4581 & 1.3778 & 0.4286 & -0.1851 & 0.5883 & 0.2844 & -0.1359 & 0.3740 & 0.2019 & -0.0979 & 0.2615 & 0.1474 & -0.0715 \\
\hline 0.4686 & 1.3689 & 0.4268 & -0.2744 & 0.5804 & 0.2789 & -0.1928 & 0.3679 & 0.1966 & -0.1362 & 0.2569 & 0.1430 & -0.0986 \\
\hline 0.4779 & 1.3564 & 0.4256 & -0.4008 & 0.5692 & 0.2712 & -0.2668 & 0.3593 & 0.1892 & -0.1838 & 0.2506 & 0.1371 & -0.1316 \\
\hline 0.4861 & 1.3378 & 0.4215 & -0.5930 & 0.5530 & 0.2590 & -0.3688 & 0.3472 & 0.1788 & -0.2466 & 0.2416 & 0.1292 & -0.1743 \\
\hline 0.4935 & 1.3058 & 0.3760 & -0.8987 & 0.5270 & 0.2268 & -0.5179 & 0.3286 & 0.1574 & -0.3348 & 0.2281 & 0.1144 & -0.2340 \\
\hline 0.5000 & 1.1304 & 0.3266 & -7.0017 & 0.4315 & 0.2035 & -2.8799 & 0.2670 & 0.1510 & -1.7848 & 0.1849 & 0.1122 & -1.2363 \\
\hline
\end{tabular}


Table 3.11 Normalized $K_{1}, T_{11}$ and $T_{33}$ for $\mathrm{a} / \mathrm{W}=0.2, \mathrm{t} / \mathrm{W}=4.0, \mathrm{SECP}$

\begin{tabular}{|c|c|c|c|c|c|c|c|c|c|c|c|c|}
\hline \multirow{2}{*}{$z / t$} & \multicolumn{3}{|c|}{ Uniform } & \multicolumn{3}{|c|}{ Linear } & \multicolumn{3}{|c|}{ Parabolic } & \multicolumn{3}{|c|}{ Cubic } \\
\hline & $K_{I}$ & $T_{11}$ & $T_{33}$ & $K_{I}$ & $T_{11}$ & $T_{33}$ & $K_{1}$ & $T_{n}$ & $T_{33}$ & $K_{I}$ & $T_{l l}$ & $T_{33}$ \\
\hline 0.0000 & 1.3672 & 0.4075 & 0.1211 & 0.5855 & 0.2707 & 0.0805 & 0.3731 & 0.1930 & 0.0574 & 0.2613 & 0.1412 & 0.0420 \\
\hline 0.0615 & 1.3673 & 0.4075 & 0.1210 & 0.5856 & 0.2707 & 0.0805 & 0.3731 & 0.1930 & 0.0574 & 0.2613 & 0.1412 & 0.0420 \\
\hline 0.1161 & 1.3676 & 0.4075 & 0.1208 & 0.5858 & 0.2707 & 0.0803 & 0.3733 & 0.1929 & 0.0573 & 0.2614 & 0.1412 & 0.0419 \\
\hline 0.1647 & 1.3681 & 0.4075 & 0.1204 & 0.5861 & 0.2707 & 0.0801 & 0.3735 & 0.1929 & 0.0571 & 0.2616 & 0.1412 & 0.0418 \\
\hline 0.2078 & 1.3690 & 0.4076 & 0.1200 & 0.5866 & 0.2707 & 0.0798 & 0.3739 & 0.1930 & 0.0569 & 0.2618 & 0.1412 & 0.0417 \\
\hline 0.2462 & 1.3702 & 0.4080 & 0.1194 & 0.5874 & 0.2710 & 0.0794 & 0.3744 & 0.1932 & 0.0566 & 0.2622 & 0.1414 & 0.0415 \\
\hline 0.2802 & 1.3719 & 0.4090 & 0.1186 & 0.5885 & 0.2716 & 0.0789 & 0.3752 & 0.1936 & 0.0563 & 0.2627 & 0.1417 & 0.0412 \\
\hline 0.3105 & 1.3742 & 0.4106 & 0.1173 & 0.5899 & 0.2726 & 0.0781 & 0.3761 & 0.1943 & 0.0557 & 0.2635 & 0.1422 & 0.0408 \\
\hline 0.3374 & 1.3769 & 0.4132 & 51 & 916 & 0.2742 & 767 & 0.3773 & 0.1954 & 0.0547 & 0.2643 & 0.1430 & 0.0400 \\
\hline 0.3613 & 1.3800 & 0.4170 & 0.1113 & 0.5936 & 0.2766 & 0.0741 & 0.3787 & 0.1971 & 0.0529 & 0.2653 & 0.1442 & 0.038 \\
\hline 0.3825 & 1.3832 & 0.4220 & 0.1047 & 0.5957 & 0.2799 & 0.0697 & 0.3802 & \begin{tabular}{|l|l|}
0.1994 \\
\end{tabular} & 0.0497 & 0.2664 & 0.1459 & 0.036 \\
\hline 0.4014 & 1.3863 & 0.4278 & 0.0940 & 0.5977 & 0.2839 & 0.0624 & 0.3816 & 0.2023 & 0.0445 & 0.2674 & 0.1480 & 0.0326 \\
\hline 0.4182 & 1.3889 & 0.4336 & 0.0780 & 0.5993 & 0.2882 & 0.0512 & 0.3827 & 0.2054 & 0.0364 & 0.2682 & 0.1503 & 0.0266 \\
\hline 0.4331 & 1.3904 & 0.4381 & 0.0552 & 0.6003 & 0.2919 & 0.0349 & 0.3834 & 0.2082 & 0.0245 & 0.2687 & 0.1524 & 0.0179 \\
\hline 0.4464 & 1.3906 & 0.4399 & 0.0242 & 0.6002 & 0.2940 & 0.0122 & 0.3833 & 0.2100 & 0.0079 & 0.2686 & 0.1538 & 0.0056 \\
\hline 0.4581 & 1.3889 & 0.4382 & -0.0172 & 0.5987 & 0.2938 & -0.0187 & 0.3821 & 0.2100 & -0.0147 & 0.2677 & 0.1538 & -0.0112 \\
\hline 0.4686 & 1.3845 & 0.4337 & -0.0732 & 0.5950 & 0.2909 & -0.0606 & 0.3793 & 0.2077 & -0.0452 & 0.2656 & 0.1521 & -0.0336 \\
\hline 0.4779 & 1.3764 & 0.4273 & -0.1527 & 0.5879 & 0.2849 & -0.1186 & 0.3738 & 0.2025 & -0.0864 & 0.2614 & 0.1480 & -0.0634 \\
\hline 0.4861 & 1.3620 & 0.4192 & -0.2746 & 0.5750 & 0.2738 & -0.2019 & 0.3638 & 0.1926 & -0.1426 & 0.2539 & 0.1400 & -0.1031 \\
\hline 0.4935 & 1.3350 & 0.3888 & -0.4806 & 0.5503 & 0.2453 & -0.3282 & 0.3452 & 0.1701 & -0.2213 & 0.2401 & 0.1231 & -0.1567 \\
\hline 0.5000 & 1.1612 & 0.3779 & -5.3364 & 0.4444 & 0.2189 & -2.2432 & 0.2748 & 0.1591 & -1.3910 & 0.1903 & 0.1176 & -0.9634 \\
\hline
\end{tabular}


Table 3.12 Normalized $K_{\mathrm{l}}, T_{11}$ and $T_{33}$ for $\mathrm{a} / \mathrm{W}=0.4, \mathrm{t} / \mathrm{W}=0.1$, SECP

\begin{tabular}{|c|c|c|c|c|c|c|c|c|c|c|c|c|}
\hline \multirow{2}{*}{$z / t$} & \multicolumn{3}{|c|}{ Uniform } & \multicolumn{3}{|c|}{ Linear } & \multicolumn{3}{|c|}{ Parabolic } & \multicolumn{3}{|c|}{ Cubic } \\
\hline & $K_{l}$ & $T_{l l}$ & $T_{33}$ & $K_{I}$ & $T_{l l}$ & $T_{33}$ & $K_{I}$ & $T_{l l}$ & $T_{33}$ & $K_{I}$ & $T_{n}$ & $T_{33}$ \\
\hline 0.0000 & 2.2452 & 0.6641 & -2.0153 & 1.1124 & 0.4148 & -1.2047 & 0.7479 & 0.2961 & -0.8261 & 0.5486 & 0.2235 & -0.6082 \\
\hline 0.0346 & 2.2446 & 0.6639 & -2.0228 & 1.1122 & 0.4146 & -1.2086 & 0.7478 & 0.2960 & -0.8287 & 0.5485 & 0.2235 & -0.6101 \\
\hline 0.0679 & 2.2440 & 0.6655 & -2.0444 & 1.1118 & 0.4152 & -1.2197 & 0.7475 & 0.2965 & -0.8360 & 0.5483 & 0.2238 & -0.6155 \\
\hline 0.1001 & 2.2427 & 0.6679 & -2.0792 & 1.1110 & 0.4163 & -1.2377 & 0.7469 & 0.2972 & -0.8479 & 0.5479 & 0.2244 & -0.6242 \\
\hline 0.1311 & 2.2414 & 0.6712 & -2.1271 & 1.1100 & 0.4176 & -1.2624 & 0.7462 & 0.2982 & -0.8643 & 5474 & 0.2252 & -0.6361 \\
\hline 0.1610 & 2.2395 & 0. & -2.1883 & 86 & 0.4 & 38 & 0.7453 & 95 & 51 & 467 & 261 & -0. \\
\hline 0.1898 & 2.2369 & 0.6801 & -2.2633 & 70 & 0.4214 & -1.3323 & 0.7442 & 0.3009 & -0.9107 & 0.5459 & 0.2273 & -0.6700 \\
\hline 0.2176 & 2.2337 & 0. & -2.35 & 50 & 237 & 785 & 429 & 0.3026 & -0.9413 & 449 & 0.2286 & -0.6924 \\
\hline 0.2444 & 2.2304 & 0. & -2 . & 27 & 3 & 330 & 414 & 46 & 75 & 438 & 301 & \\
\hline 0.2702 & 2.2259 & 0.6988 & -2.5865 & 1.1000 & 0.4292 & -1.4972 & 0.7395 & 0.3067 & -1.0202 & 0.5425 & 0.2317 & -0.7501 \\
\hline 0.2951 & 2.2207 & 0.7065 & -2.7355 & 1.0968 & 0.4325 & -1.5728 & 0.7374 & 0.3091 & -1.0705 & 0.5409 & 0.2336 & -0.7870 \\
\hline 0.3191 & 2.2142 & 0.7149 & -2.9126 & 1.0931 & 0.4360 & -1.6623 & 0.7349 & 0.3118 & -1.1301 & 0.5391 & 0.2356 & -0.8306 \\
\hline 0.3423 & 2.2070 & 0.7241 & -3.1253 & 1.0887 & 0.4400 & -1.7695 & 0.7320 & 0.3148 & -1.2015 & 0.5370 & 0.2379 & -0.8829 \\
\hline 0.3646 & 2.1985 & 0.7342 & -3.3851 & 1.0835 & 0.4443 & -1.8997 & 0.7285 & 0.3180 & -1.2884 & 0.5345 & 0.2404 & -0.9466 \\
\hline 0.3861 & 2.1880 & 0.7452 & -3.7089 & 1.0773 & 0.4491 & -2.0615 & 0.7244 & 0.3217 & -1.3964 & 0.5315 & 0.2431 & -1.0258 \\
\hline 0.4069 & 2.1748 & 0.7566 & -4.1230 & 1.0697 & 0.4541 & -2.2674 & 0.7193 & 0.3254 & -1.5342 & 0.5278 & 0.2460 & -1.1268 \\
\hline 0.4269 & 2.1575 & 0.7643 & -4.6635 & 1.0602 & 0.4573 & -2.5353 & 0.7130 & 0.3281 & -1.7136 & 0.5232 & 0.2481 & -1.2584 \\
\hline 0.4462 & 2.1354 & 0.7494 & -5.3742 & 1.0478 & 0.4497 & -2.8864 & 0.7048 & 0.3236 & -1.9489 & 0.5172 & 0.2448 & -1.4310 \\
\hline 0.4648 & 2.1048 & 0.6120 & -6.2272 & 1.0309 & 0.3825 & -3.3073 & 0.6935 & 0.2791 & -2.2311 & 0.5090 & 0.2122 & -1.6381 \\
\hline 0.4827 & 2.0612 & -0.1896 & -6.6403 & 1.0075 & -0.0078 & -3.5143 & 0.6779 & 0.0172 & -2.3691 & 0.4976 & 0.0200 & -1.7393 \\
\hline 0.5000 & 1.8103 & 0.1414 & 29.2747 & 0.8817 & 0.1650 & -14.5408 & 0.5936 & 0.1337 & -9.7924 & 0.4358 & 0.1052 & -7.1886 \\
\hline
\end{tabular}


Table 3.13 Normalized $K_{1}, T_{11}$ and $T_{33}$ for $\mathrm{a} / \mathrm{W}=0.4, \mathrm{t} / \mathrm{W}=0.2$, SECP

\begin{tabular}{|c|c|c|c|c|c|c|c|c|c|c|c|c|}
\hline \multirow{2}{*}{$z / t$} & \multicolumn{3}{|c|}{ Uniform } & \multicolumn{3}{|c|}{ Linear } & \multicolumn{3}{|c|}{ Parabolic } & \multicolumn{3}{|c|}{ Cubic } \\
\hline & $K_{I}$ & $T_{l l}$ & $T_{33}$ & $K_{I}$ & $T_{l l}$ & $T_{33}$ & $K_{I}$ & $T_{l l}$ & $T_{33}$ & $K_{I}$ & $T_{l l}$ & $T_{33}$ \\
\hline 0.0000 & 2.2407 & 0.5943 & -1.2232 & 1.1144 & 0.3859 & -0.7767 & 0.7498 & 0.2775 & -0.5444 & 0.5501 & 0.2096 & -0.4039 \\
\hline 0.0490 & 2.2407 & 0.5951 & -1.2350 & 1.1140 & 0.3861 & -0.7831 & 0.7495 & 0.2776 & -0.5486 & 0.5498 & 0.2097 & -0.4069 \\
\hline 0.0946 & 2.2388 & 0.5966 & -1.2637 & 1.1129 & 0.3864 & -0.7987 & 0.7488 & 0.2778 & -0.5588 & 0.5493 & 0.2098 & -0.4143 \\
\hline 0.1361 & 2.2369 & 0.5999 & -1.3102 & 1.1112 & 0.3874 & -0.8238 & 0.7475 & 0.2784 & -0.5752 & 0.5483 & 0.2103 & -0.4262 \\
\hline 0.1747 & 2.2337 & 0.6033 & -1.3700 & 1.1090 & 0.3882 & -0.8559 & 0.7459 & 0.2788 & -0.5962 & 0.5472 & 0.2107 & -0.4414 \\
\hline 0.2098 & 2.2304 & 0.6081 & -1.4451 & 1.1062 & 0.3896 & -0.8961 & 0.7439 & 0.2798 & -0.6226 & 0.5457 & 0.2114 & -0.4605 \\
\hline 0.2424 & 2.2259 & 0.6126 & -1.5329 & 1.1030 & 0.3907 & -0.9427 & 0.7417 & 0.2805 & -0.6532 & 0.5440 & 0.2121 & -0.4827 \\
\hline 0.2722 & 2.2207 & 0.6186 & -1.6367 & 1.0992 & 0.3924 & -0.9975 & 0.7390 & 0.2817 & \begin{tabular}{|c|c|}
-0.6891 \\
\end{tabular} & 0.5420 & 0.2131 & -0.5088 \\
\hline 0.2998 & 2.2142 & 0.6237 & -1.7550 & 1.0950 & 0.3936 & -1.0594 & 0.7360 & 0.2827 & -0.7299 & 0.5398 & 0.2139 & -0.5384 \\
\hline 0.3250 & 2.2077 & 0.6302 & -1.8927 & 1.0901 & 0.3955 & -1.1310 & 0.7326 & 0.2841 & -0.7770 & 0.5374 & 0.2151 & -0.5727 \\
\hline 0.3484 & 2.1992 & 0.6356 & -2.0493 & 1.0847 & 0.3968 & -1.2117 & 0.7289 & 0.2853 & -0.8302 & 0.5346 & 0.2162 & -0.6115 \\
\hline 0.3698 & 2.1900 & 0.6425 & -2.2330 & 1.0786 & 0.3989 & -1.3057 & 0.7247 & 0.2870 & -0.8922 & 0.5315 & 0.2176 & -0.6567 \\
\hline 0.3896 & 2.1794 & 0.6473 & -2.4456 & 1.0717 & 0.3999 & -1.4136 & 0.7200 & 0.2882 & -0.9636 & 0.5281 & 0.2187 & -0.7089 \\
\hline 0.4076 & 2.1668 & 0.6542 & -2.7013 & 1.0638 & 0.4020 & -1.5423 & 0.7146 & 0.2901 & -1.0489 & 0.5241 & 0.2203 & -0.7712 \\
\hline 0.4244 & 2.1528 & 0.6577 & -3.0108 & 1.0549 & 0.4025 & -1.6970 & 0.7086 & 0.2911 & -1.1517 & 0.5197 & 0.2213 & -0.8464 \\
\hline 0.4397 & 2.1354 & 0.6640 & -3.3987 & 1.0444 & 0.4044 & -1.8894 & 0.7015 & 0.2932 & $\begin{array}{l}-1.2797 \\
\end{array}$ & 0.5145 & 0.2231 & -0.9402 \\
\hline 0.4539 & 2.1143 & 0.6656 & -3.9281 & 1.0320 & 0.4043 & -2.1501 & 0.6931 & 0.2940 & -1.4536 & 0.5085 & 0.2239 & -1.0676 \\
\hline 0.4668 & 2.0862 & 0.6679 & -4.6275 & 1.0160 & 0.4048 & -2.4926 & 0.6825 & 0.2954 & -1.6824 & 0.5007 & 0.2252 & -1.2353 \\
\hline 0.4789 & 2.0500 & 0.6544 & -5.8780 & 0.9956 & 0.3982 & -3.1011 & 0.6688 & 0.2921 & -2.0899 & 0.4907 & 0.2230 & -1.5342 \\
\hline 0.4898 & 1.9791 & 0.6529 & -8.8721 & 0.9582 & 0.3995 & -4.5486 & 0.6439 & 0.2945 & -3.0614 & 0.4724 & 0.2249 & -2.2471 \\
\hline 0.5000 & 1.7222 & -0.1601 & -30.1771 & 0.8291 & 0.0242 & -14.8093 & 0.5574 & 0.0431 & -9.9582 & 0.4091 & 0.0403 & -7.3088 \\
\hline
\end{tabular}


Table 3.14 Normalized $K_{1}, T_{11}$ and $T_{33}$ for $\mathrm{a} / \mathrm{W}=0.4, \mathrm{t} / \mathrm{W}=0.5$, SECP

\begin{tabular}{|c|c|c|c|c|c|c|c|c|c|c|c|c|}
\hline \multirow{2}{*}{$z / t$} & \multicolumn{3}{|c|}{ Uniform } & \multicolumn{3}{|c|}{ Linear } & \multicolumn{3}{|c|}{ Parabolic } & \multicolumn{3}{|c|}{ Cubic } \\
\hline & $K_{I}$ & $T_{11}$ & $T_{33}$ & $K_{I}$ & $T_{11}$ & $T_{33}$ & $K_{I}$ & $T_{n}$ & $T_{33}$ & $K_{I}$ & $\overline{T_{11}}$ & $T_{33}$ \\
\hline 0.0000 & 2.2272 & 0.5467 & -0.5103 & 1.1137 & 0.3725 & -0.3454 & 0.7514 & 0.2745 & -0.2518 & 0.5520 & 0.2097 & -0.1908 \\
\hline 0.0615 & 2.2265 & 0.5463 & -0.5211 & 1.1130 & 0.3718 & -0.3523 & 0.7509 & 0.2738 & -0.2565 & 0.5515 & 0.2091 & -0.1943 \\
\hline 0.1161 & 2.2240 & 0.5458 & -0.5497 & 1.1110 & 0.3705 & -0.3703 & 0.7494 & 0.2724 & -0.2690 & 0.5504 & 0.2079 & -0.2034 \\
\hline 0.1647 & 2.2201 & 0.5452 & -0.5927 & 1.1081 & 0.3686 & -0.3971 & 0.7471 & 0.2704 & -0.2873 & 0.5486 & 0.2061 & -0.2169 \\
\hline 0.2078 & 2.2155 & 0. & -0.64 & 043 & 0.3663 & 312 & 0.7441 & 0.2680 & -0.3106 & 5463 & 0.2040 & 338 \\
\hline 0.2462 & 2.2097 & 0.5447 & -0.7149 & 1.0996 & 0.3638 & -0.4717 & 0.7405 & 0.2653 & -0.3379 & 0.5435 & 0.2017 & -0.2536 \\
\hline 0.2802 & 2.2031 & 0. & -0.7931 & 1. & 611 & -0.5181 & 0.7364 & 625 & 90 & 403 & 992 & 762 \\
\hline 0.3105 & 2.1953 & 6 & -0. & 1. & 33 & 3 & 17 & 96 & 38 & 67 & 968 & 013 \\
\hline 0.3374 & 2.1 & 0 & & & 0 & & 55 & 57 & 24 & 27 & 944 & 3291 \\
\hline 0.3613 & 2.1768 & 0.5477 & -1.1012 & 1.0735 & 0.3526 & -0.6934 & 0.7208 & 0.2539 & -0.4850 & 0.5283 & 0.1922 & -0.3599 \\
\hline 0.3825 & 2.1655 & 0.5490 & -1.2335 & 1.0650 & 0.3497 & -0.7657 & 0.7146 & 0.2513 & -0.5324 & 0.5236 & 0.1901 & -0.3941 \\
\hline 0.4014 & 2.1528 & 0.5504 & -1.3853 & 1.0558 & 0.3467 & -0.8469 & 0.7078 & 0.2488 & -0.5855 & 0.5185 & 0.1883 & -0.4324 \\
\hline 0.4182 & 2.1387 & 0.5516 & -1.5614 & 1.0457 & 0.3437 & -0.9393 & 0.7005 & 0.2466 & -0.6459 & 0.5130 & 0.1867 & -0.4761 \\
\hline 0.4331 & 2.1232 & 0.5527 & -1.7692 & 1.0346 & 0.3407 & -1.0464 & 0.6925 & 0.2445 & -0.7159 & 0.5070 & 0.1854 & -0.5268 \\
\hline 0.4464 & 2.1055 & 0.5533 & -2.0204 & 1.0223 & 0.3376 & -1.1735 & 0.6838 & 0.2427 & -0.7991 & 0.5006 & 0.1843 & -0.5871 \\
\hline 0.4581 & 2.0848 & 0.5533 & -2.3347 & 1.0085 & 0.3343 & -1.3301 & 0.6741 & 0.2411 & -0.9017 & 0.4934 & 0.1835 & -0.6618 \\
\hline 0.4686 & 2.0605 & 0.5528 & -2.7489 & 0.9926 & 0.3312 & -1.5334 & 0.6632 & 0.2399 & -1.0354 & 0.4853 & 0.1830 & -0.7592 \\
\hline 0.4779 & 2.0295 & 0.5513 & -3.3409 & 0.9737 & 0.3281 & -1.8201 & 0.6503 & 0.2391 & -1.2246 & 0.4758 & 0.1829 & -0.8972 \\
\hline 0.4861 & 1.9893 & 0.5455 & -4.2918 & 0.9495 & 0.3237 & -2.2756 & 0.6339 & 0.2379 & -1.5264 & 0.4638 & 0.1825 & -1.1176 \\
\hline 0.4935 & 1.9260 & 0.3934 & -5.8885 & 0.9141 & 0.2526 & -3.0352 & 0.6101 & 0.1928 & -2.0307 & 0.4464 & 0.1500 & -1.4863 \\
\hline 0.5000 & 1.6405 & -0.0935 & 27.2184 & 0.7711 & 0.0458 & -13.0640 & 0.5148 & 0.0582 & -8.7222 & 0.3767 & 0.0520 & -6.3823 \\
\hline
\end{tabular}

52 
Table 3. 15 Normalized $K_{1}, T_{11}$ and $T_{33}$ for a/W=0.4, $t / \mathrm{W}=1.0$, SECP

\begin{tabular}{|c|c|c|c|c|c|c|c|c|c|c|c|c|}
\hline \multirow{2}{*}{$z / t$} & \multicolumn{3}{|c|}{ Uniform } & \multicolumn{3}{|c|}{ Linear } & \multicolumn{3}{|c|}{ Parabolic } & \multicolumn{3}{|c|}{ Cubic } \\
\hline & $K_{I}$ & $T_{l l}$ & $T_{33}$ & $K_{I}$ & $T_{l l}$ & $T T_{33}$ & $K_{I}$ & $T_{l l}$ & $T_{33}$ & $\bar{K}$ & $T_{11}$ & $T_{33}$ \\
\hline 0.0000 & 2.1966 & 0.5299 & -0.1172 & 1.0969 & 0.3605 & -0.0776 & 0.7404 & 0.2663 & -0.0562 & 0.5441 & 0.2038 & -0.0425 \\
\hline 0.0615 & 2.1959 & 0.5288 & -0.1260 & 1.0965 & 0.3598 & -0.0836 & 0.7401 & 0.2658 & -0.0607 & 0.5438 & 0.2034 & -0.0459 \\
\hline 0.1161 & 2.1946 & 0.5262 & -0.1489 & 1.0953 & 0.3582 & -0.0995 & 0.7392 & 0.2646 & -0.0724 & 0.5431 & 0.2025 & -0.0548 \\
\hline 0.1647 & 2.1920 & 0.5222 & -0.1830 & 1.0934 & 0.3556 & -0.1229 & 0.7377 & 0.2627 & -0.0896 & 0.5420 & 0.2010 & -0.0679 \\
\hline 0.2078 & 2.1880 & 0.5172 & -0.2262 & 1.0908 & 0.3521 & -0.1525 & 0.7357 & 0.2600 & -0.1112 & 0.5404 & 0.1989 & -0.0843 \\
\hline 0.2462 & 2.1841 & 0.5112 & -0.2777 & 1.0873 & 0.3477 & -0.1874 & 0.7330 & 0.2565 & -0.1365 & 0.5383 & 0.1961 & -0.1034 \\
\hline 0.2802 & 2.1781 & 0.5047 & 370 & 1.0830 & 0.3426 & -0.2271 & 0.7297 & 0.2523 & -0.1650 & 0.5357 & 0.1927 & -0.1248 \\
\hline 0.3105 & 2.1715 & 0.4980 & -0.4042 & 1.0777 & 0.3368 & -0.2713 & 0.7257 & 0.2474 & -0.1965 & 0.5326 & 0.1888 & -0.1483 \\
\hline 0.3374 & 2.1635 & 0.4913 & -0.4798 & 1.0715 & 0.3305 & -0.3201 & 0.7209 & 0.2421 & -0.2307 & 0.5289 & 0.1844 & -0.1736 \\
\hline 0.3613 & 2.1542 & 0.4849 & -0.5648 & 1.0644 & 0.3238 & -0.3736 & 0.7154 & 0.2362 & -0.2677 & 0.5246 & 0.1796 & -0.2009 \\
\hline 0.3825 & 2.1434 & 0.4788 & -0.6609 & 1.0561 & 0.3168 & -0.4324 & 0.7091 & 0.2301 & -0.3078 & 0.5197 & 0.1746 & -0.2302 \\
\hline 0.4014 & 2.1313 & 0.4733 & -0.7704 & 1.0468 & 0.3095 & -0.4974 & 0.7020 & 0.2237 & -0.3516 & 0.5143 & 0.1694 & -0.2620 \\
\hline 0.4182 & 2.1177 & 0.4682 & -0.8969 & 1.0363 & 0.3019 & -0.5701 & 0.6941 & 0.2172 & -0.4000 & 0.5082 & 0.1642 & -0.2970 \\
\hline 0.4331 & 2.1020 & 0.4635 & -1.0457 & 1.0245 & 0.2942 & -0.6530 & 0.6853 & 0.2108 & -0.4545 & 0.5015 & 0.1592 & -0.3364 \\
\hline 0.4464 & 2.0841 & 0.4592 & -1.2250 & 1.0112 & 0.2864 & -0.7497 & 0.6755 & 0.2045 & -0.5177 & 0.4940 & 0.1544 & -0.3819 \\
\hline 0.4581 & 2.0633 & 0.4549 & -1.4485 & 0.9961 & 0.2784 & -0.8665 & 0.6645 & 0.1985 & -0.5937 & 0.4857 & 0.1500 & -0.4367 \\
\hline 0.4686 & 2.0387 & 0.4504 & -1.7389 & 0.9787 & 0.2702 & -1.0140 & 0.6520 & 0.1928 & -0.6895 & 0.4763 & 0.1460 & -0.5059 \\
\hline 0.4779 & 2.0081 & 0.4427 & -2.1386 & 0.9579 & 0.2606 & -1.2118 & 0.6372 & 0.1868 & -0.8181 & 0.4654 & 0.1420 & -0.5990 \\
\hline 0.4861 & 1.9675 & 0.4194 & -2.7289 & 0.9314 & 0.2443 & -1.4976 & 0.6189 & 0.1773 & -1.0046 & 0.4518 & 0.1357 & -0.7344 \\
\hline 0.4935 & 1.9042 & 0.2680 & -3.6400 & 0.8930 & 0.1703 & -1.9322 & 0.5927 & 0.1308 & -1.2889 & 0.4326 & 0.1025 & -0.9412 \\
\hline 0.5000 & 1.6231 & 0.1351 & -21.0594 & 0.7488 & 0.1277 & -9.9763 & 0.4967 & 0.1085 & -6.6200 & 0.3625 & 0.0875 & -4.8304 \\
\hline
\end{tabular}


Table 3.16 Normalized $K_{1}, T_{11}$ and $T_{33}$ for $\mathrm{a} / \mathrm{W}=0.4, \mathrm{t} / \mathrm{W}=2.0, \mathrm{SECP}$

\begin{tabular}{|c|c|c|c|c|c|c|c|c|c|c|c|c|}
\hline \multirow{2}{*}{$z / t$} & \multicolumn{3}{|c|}{ Uniform } & \multicolumn{3}{|c|}{ Linear } & \multicolumn{3}{|c|}{ Parabolic } & \multicolumn{3}{|c|}{ Cubic } \\
\hline & $\boldsymbol{K}_{I}$ & $T_{11}$ & $T_{33}$ & $K_{I}$ & $T_{I I}$ & $T_{33}$ & $\boldsymbol{K}_{I}$ & $T_{11}$ & $T_{33}$ & $K_{I}$ & $T_{l l}$ & $T_{33}$ \\
\hline 0.0000 & 2.1481 & 0.4385 & 0.0997 & 1.0660 & 0.2984 & 0.0682 & 0.7182 & 0.2206 & 0.0506 & 0.5273 & 0.1689 & 0.0388 \\
\hline 0.0615 & 2.1488 & 0.4411 & 0.0952 & 1.0663 & 0.3001 & 0.0652 & 0.7184 & 0.2218 & 0.0484 & 0.5275 & 0.1699 & 0.0371 \\
\hline 0.1161 & 2.1502 & 0.4476 & 0.0828 & 1.0670 & 0.3045 & 0.0570 & 0.7189 & 0.2250 & 0.0424 & 0.5278 & 0.1723 & 0.0326 \\
\hline 0.1647 & 2.1508 & 0.4564 & 0.0631 & 1.0677 & 0.3104 & 0.0438 & 0.7194 & 0.2294 & 0.0327 & 0.5283 & 0.1757 & 0.0252 \\
\hline 0.2078 & 2.1522 & 0.4658 & 0.0362 & 1.0683 & 0.3169 & 0.0257 & 0.7199 & 0.2342 & 0.0195 & 0.5286 & 0.1793 & 0.0151 \\
\hline 0.2462 & 2.1522 & 0.4743 & 0.0023 & 1.0686 & 0.3228 & 0.0027 & 0.7201 & 0.2386 & 0.0026 & 0.5287 & 0.1827 & 0.0022 \\
\hline 0.2802 & 2.1522 & 0.4809 & -0.0385 & 1.0683 & 0.3276 & -0.0251 & 0.7198 & 0.2422 & -0.0179 & 0.5285 & 0.1855 & -0.0134 \\
\hline 0.3105 & 2.1508 & 0.4849 & -0.0863 & 1. & 306 & -0 . & 189 & 0.2444 & 420 & 278 & 872 & -0. \\
\hline 0.3374 & 2. & 0. & -0 & 1 & 316 & 954 & 173 & 0.2451 & -0.0695 & 5266 & 0.1877 & -0.0527 \\
\hline 0.3613 & 2. & 0.4 & 38 & 1 & 0 & 79 & 149 & 41 & -0.1006 & 5247 & 1868 & -0.0762 \\
\hline 0.3825 & 2.1381 & 0.4814 & -0.2748 & 1.0576 & 0.3277 & -0.1858 & 0.7115 & 0.2416 & -0.1351 & 0.5220 & 0.1848 & -0.1023 \\
\hline 0.4014 & 2.1306 & 0.4765 & -0.3558 & 1.0517 & 0.3231 & -0.2394 & 0.7070 & 0.2377 & -0.1735 & 0.5185 & 0.1815 & -0.1310 \\
\hline 0.4182 & 2.1211 & 0.4707 & -0.4488 & 1.0443 & 0.3171 & -0.2996 & 0.7013 & 0.2324 & -0.2159 & 0.5141 & 0.1770 & -0.1624 \\
\hline 0.4331 & 2.1096 & 0.4643 & -0.5572 & 1.0351 & 0.3098 & -0.3677 & 0.6942 & 0.2258 & -0.2630 & 0.5086 & 0.1716 & -0.1971 \\
\hline 0.4464 & 2.0945 & 0.4576 & -0.6862 & 1.0239 & 0.3012 & -0.4460 & 0.6857 & 0.2183 & -0.3161 & 0.5019 & 0.1654 & -0.2358 \\
\hline 0.4581 & 2.0772 & 0.4505 & -0.8439 & 1.0102 & 0.2913 & -0.5379 & 0.6753 & 0.2097 & -0.3774 & 0.4940 & 0.1586 & -0.2802 \\
\hline 0.4686 & 2.0549 & 0.4428 & -1.0441 & 0.9934 & 0.2800 & -0.6497 & 0.6627 & 0.2004 & -0.4508 & 0.4844 & 0.1514 & -0.3330 \\
\hline 0.4779 & 2.0266 & 0.4317 & -1.3132 & 0.9723 & 0.2673 & -0.7123 & 0.6473 & 0.1898 & -0.5440 & 0.4727 & 0.1435 & -0.4001 \\
\hline 0.4861 & 1.9871 & 0.4086 & -1.7074 & 0.9445 & 0.2457 & -0.9952 & 0.6273 & 0.1761 & -0.6742 & 0.4577 & 0.1338 & -0.4939 \\
\hline 0.4935 & 1.9253 & 0.3052 & -2.3487 & 0.9036 & 0.1883 & -1.3116 & 0.5988 & 0.1398 & -0.8784 & 0.4366 & 0.1082 & -0.6415 \\
\hline 0.5000 & 1.6404 & 0.2603 & 16.0124 & 0.7507 & 0.1775 & -7.5829 & 0.4966 & 0.1408 & -5.0185 & 0.3619 & 0.1109 & -3.6573 \\
\hline
\end{tabular}


Table 3.17 Normalized $K_{\mathrm{l}}, T_{11}$ and $T_{33}$ for $\mathrm{a} / \mathrm{W}=0.4, \mathrm{t} / \mathrm{W}=4.0$, SECP

\begin{tabular}{|c|c|c|c|c|c|c|c|c|c|c|c|c|}
\hline \multirow{2}{*}{$z / t$} & \multicolumn{3}{|c|}{ Uniform } & \multicolumn{3}{|c|}{ Linear } & \multicolumn{3}{|c|}{ Parabolic } & \multicolumn{3}{|c|}{ Cubic } \\
\hline & $K_{l}$ & $T_{l I}$ & $T_{33}$ & $\overline{K_{I}}$ & $T_{l l}$ & $T_{33}$ & $K_{I}$ & $T_{l l}$ & $T_{33}$ & $K_{I}$ & $T_{l l}$ & $T_{33}$ \\
\hline 0.0000 & 2.1218 & 0.4209 & 0.1244 & 1.0486 & 0.2868 & 0.0847 & 0.7057 & 0.2122 & 0.0626 & 0.5178 & 0.1626 & 0.0480 \\
\hline 0.0615 & 2.1218 & 0.4209 & 0.1242 & 1.0488 & 0.2868 & 0.0846 & 0.7058 & 0.2122 & 0.0625 & 0.5179 & 0.1626 & 0.0479 \\
\hline 0.1161 & 2.1225 & 0.4211 & 0.1237 & 1.0493 & 0.2869 & 0.0842 & 0.7061 & 0.2123 & 0.0623 & 0.5182 & 0.1626 & 0.0477 \\
\hline 0.1647 & 2.1239 & 0.4220 & 0.1225 & 1.0501 & 0.2875 & 0.0834 & 0.7067 & 0.2127 & 0.0616 & 0.5186 & 0.1630 & 0.0472 \\
\hline 0.2078 & 2.1252 & 0.4245 & 0.1196 & 1.0512 & 0.2892 & 0.0814 & 0.7075 & 0.2139 & 0.0602 & 0.5192 & 639 & 0.0461 \\
\hline 0.2462 & 2.1272 & 0.4292 & 0.1137 & 1.0525 & 0.2922 & 0.0775 & 0.7085 & 0.2161 & 0.0574 & 0.5200 & 0.1656 & 0.0440 \\
\hline 0.2802 & 2.1300 & 0.4366 & 0.1033 & 1.0541 & 0.2972 & 0.0705 & 0.7096 & 0.2197 & 0.0523 & 0.5209 & 683 & 0.0401 \\
\hline 0.3105 & 2.1327 & 0.4464 & 0.0866 & 1.0557 & 0.3037 & 0.0593 & 0.7108 & 0.2245 & 0.0441 & 0.5218 & 0.1719 & 0.0339 \\
\hline 0.3374 & 2.1347 & 0.4577 & 0.0621 & 1.0572 & 0.3113 & 0.0429 & 0.7119 & 0.2301 & 0.0320 & 0.5225 & 0.1762 & 0.0247 \\
\hline 0.3613 & 2.1360 & 0.4688 & 0.0288 & 1.0582 & 0.3190 & 0.0203 & 0.7126 & 0.2358 & 0.0154 & 0.5231 & 0.1805 & 0.0120 \\
\hline 0.3825 & 2.1367 & 0.4781 & -0.0139 & 1.0584 & 0.3256 & -0.0089 & 0.7127 & 0.2407 & -0.0061 & 0.5232 & 0.1843 & -0.0044 \\
\hline 0.4014 & 2.1360 & 0.4842 & -0.0668 & 1.0577 & 0.3301 & -0.0453 & 0.7121 & 0.2440 & -0.0329 & 0.5227 & 0.1869 & -0.0248 \\
\hline 0.4182 & 2.1333 & 0.4862 & -0.1307 & 1.0555 & 0.3317 & -0.0894 & 0.7104 & 0.2452 & -0.0653 & 0.5213 & 0.1877 & -0.0495 \\
\hline 0.4331 & 2.1279 & 0.4844 & -0.2075 & 1.0515 & 0.3302 & -0.1421 & 0.7073 & 0.2439 & -0.1038 & 0.5189 & 0.1866 & -0.0787 \\
\hline 0.4464 & 2.1198 & 0.4791 & -0.3001 & 1.0451 & 0.3256 & -0.2049 & 0.7024 & 0.2399 & -0.1491 & 0.5151 & 0.1833 & -0.1128 \\
\hline 0.4581 & 2.1075 & 0.4711 & -0.4138 & 1.0359 & 0.3179 & -0.2802 & 0.6953 & 0.2331 & -0.2025 & 0.5095 & 0.1777 & -0.1526 \\
\hline 0.4686 & 2.0910 & 0.4613 & -0.5579 & 1.0228 & 0.3071 & -0.3723 & 0.6852 & 0.2236 & -0.2662 & 0.5017 & 0.1699 & -0.1994 \\
\hline 0.4779 & 2.0668 & 0.4488 & -0.7516 & 1.0042 & 0.2923 & -0.4898 & 0.6711 & 0.2109 & -0.3453 & 0.4908 & 0.1596 & -0.2568 \\
\hline 0.4861 & 2.0316 & 0.4291 & -1.0372 & 0.9773 & 0.2707 & -0.6523 & 0.6510 & 0.1938 & -0.4516 & 0.4755 & 0.1464 & -0.3332 \\
\hline 0.4935 & 1.9726 & 0.3566 & -1.5102 & 0.9344 & 0.2210 & -0.9045 & 0.6200 & 0.1598 & -0.6134 & 0.4522 & 0.1219 & -0.4492 \\
\hline 0.5000 & 1.6758 & 0.3363 & -12.0742 & 0.7659 & 0.2073 & -5.7673 & 0.5062 & 0.1598 & -3.8144 & 0.3687 & 0.1246 & -2.7788 \\
\hline
\end{tabular}


Table 3.18 Normalized $K_{\mathrm{I}}, T_{11}$ and $T_{33}$ for $\mathrm{a} / \mathrm{W}=0.6, \mathrm{t} / \mathrm{W}=0.1, \mathrm{SECP}$

\begin{tabular}{|c|c|c|c|c|c|c|c|c|c|c|c|c|}
\hline \multirow{2}{*}{$z / t$} & \multicolumn{3}{|c|}{ Uniform } & \multicolumn{3}{|c|}{ Linear } & \multicolumn{3}{|c|}{ Parabolic } & \multicolumn{3}{|c|}{ Cubic } \\
\hline & $K_{I}$ & $T_{n}$ & $T_{33}$ & $K_{I}$ & $T_{l l}$ & $T_{33}$ & $K_{I}$ & $T_{11}$ & $T_{33}$ & $K_{I}$ & $T_{l 1}$ & $T_{33}$ \\
\hline 0.0000 & 4.3028 & 1.6223 & -5.2629 & 2.3459 & 0.9810 & -3.1026 & 1.5317 & 0.6531 & -2.0364 & 1.0443 & 0.4483 & -1.3892 \\
\hline 0.0346 & 4.3021 & 1.6220 & -5.2812 & 2.3455 & 0.9808 & -3.1127 & 1.5317 & 0.6530 & -2.0430 & 1.0441 & 0.4482 & -1.3937 \\
\hline 0.0679 & 4.3005 & 1.6252 & -5.3332 & 2.3447 & 0.9824 & -3.1415 & 1.5311 & 0.6541 & -2.0617 & 1.0437 & 0.4490 & -1.4065 \\
\hline 0.1001 & 4.2979 & 1.6303 & -5.4171 & 2.3430 & 0.9850 & -3.1879 & 1.5298 & 0.6558 & -2.0919 & 1.0430 & 0.4502 & -1.4271 \\
\hline 0.1311 & 4.2941 & 1.6371 & -5.5324 & 2.3406 & 0.9885 & -3.2517 & 1.5286 & 0.6582 & -2.1334 & 1.0420 & 0.4518 & -1.4553 \\
\hline 0.1610 & 4.2894 & 1.6456 & -5.6794 & 2.3377 & 0.9928 & -3.3330 & 1.5267 & 0.6611 & -2.1863 & 1.0407 & 0.4538 & -1.4914 \\
\hline 0.1898 & 4.2837 & 1.6557 & -5.8596 & 2.3344 & 0.9980 & -3.4326 & 1.5242 & 0.6645 & -2.2511 & 1.0392 & 0.4562 & -1.5355 \\
\hline 0.2176 & 4.2768 & 1.6673 & -6.0756 & 03 & 1.0039 & -3.5518 & 1.5217 & 0.6685 & -2.3287 & 1.0373 & 0.4589 & -1.5884 \\
\hline 0.2444 & 4.2685 & 1. & 313 & 253 & 07 & & 1. & & 05 & 1.0351 & 621 & -1.6510 \\
\hline 0.2702 & 4.2588 & 1.6950 & -6.6326 & 2.3195 & 1.0182 & -3.8588 & 1.5147 & 0.6781 & -2.5286 & 1.0325 & 0.4655 & -1.7246 \\
\hline 0.2951 & 4.2473 & $1.7111^{\circ}$ & -6.9877 & 2.3125 & 1.0265 & -4.0541 & 1.5102 & 0.6836 & -2.6558 & 1.0295 & 0.4693 & -1.8113 \\
\hline 0.3191 & 4.2339 & 1.7289 & -7.4087 & 2.3046 & 1.0356 & -4.2854 & 1.5051 & 0.6898 & -2.8064 & 1.0260 & 0.4736 & -1.9140 \\
\hline 0.3423 & 4.2180 & 1.7485 & -7.9132 & 2.2954 & 1.0457 & -4.5622 & 1.4988 & 0.6966 & -2.9868 & 1.0219 & 0.4783 & -2.0369 \\
\hline 0.3646 & 4.1990 & 1.7700 & -8.5276 & 2.2841 & 1.0569 & -4.8986 & 1.4917 & 0.7041 & -3.2061 & 1.0170 & 0.4834 & -2.1864 \\
\hline 0.3861 & 4.1763 & 1.7937 & -9.2916 & 2.2710 & 1.0692 & -5.3164 & 1.4833 & 0.7124 & -3.4784 & 1.0111 & 0.4891 & -2.3721 \\
\hline 0.4069 & 4.1484 & 1.8183 & -10.2658 & 2.2548 & 1.0820 & -5.8482 & 1.4722 & 0.7211 & -3.8253 & 1.0040 & 0.4951 & -2.6086 \\
\hline 0.4269 & 4.1133 & 1.8344 & -11.5348 & 2.2347 & 1.0904 & -6.5399 & 1.4591 & 0.7269 & -4.2765 & 0.9950 & 0.4990 & -2.9162 \\
\hline 0.4462 & 4.0672 & 1.7987 & -13.1998 & 2.2084 & 1.0708 & -7.4464 & 1.4426 & 0.7144 & -4.8680 & 0.9834 & 0.4906 & -3.3195 \\
\hline 0.4648 & 4.0039 & 1.4788 & -15.1967 & 2.1728 & 0.8975 & -8.5330 & 1.4191 & 0.6017 & -5.5771 & 0.9676 & 0.4137 & -3.8030 \\
\hline 0.4827 & 3.9158 & -0.3813 & -16.1726 & 2.1232 & -0.1090 & -9.0672 & 1.3869 & -0.0553 & -5.9253 & 0.9455 & -0.0343 & -4.0404 \\
\hline 0.5000 & 3.4315 & 0.4202 & -68.7319 & 1.8577 & 0.3375 & -37.5223 & 1.2136 & 0.2363 & -24.5141 & 0.8275 & 0.1644 & -16.7157 \\
\hline
\end{tabular}


Table 3.19 Normalized $K_{1}, T_{11}$ and $T_{33}$ for $\mathrm{a} / \mathrm{W}=0.6, \mathrm{t} / \mathrm{W}=0.2, \mathrm{SECP}$

\begin{tabular}{|c|c|c|c|c|c|c|c|c|c|c|c|c|}
\hline \multirow{2}{*}{$z / t$} & \multicolumn{3}{|c|}{ Uniform } & \multicolumn{3}{|c|}{ Linear } & \multicolumn{3}{|c|}{ Parabolic } & \multicolumn{3}{|c|}{ Cubic } \\
\hline & $K_{l}$ & $T_{11}$ & $T_{33}$ & $K_{I}$ & $T_{\mu \prime}$ & $T_{33}$ & $K_{I}$ & $T_{n}$ & $T_{33}$ & $K_{I}$ & $T_{11}$ & $T_{33}$ \\
\hline 0.0000 & 4.2990 & 1.4787 & -3.2914 & 2.3475 & 0.9087 & -1.9918 & 1.5330 & 0.6060 & -1.3156 & 1.0452 & 0.4160 & -0.8988 \\
\hline 0.0490 & 4.2976 & 1.4799 & -3.3209 & 2.3467 & 0.9091 & -2.0084 & 1.5323 & 0.6063 & -1.3263 & 1.0452 & 0.4162 & -0.9061 \\
\hline 0.0946 & 4.2941 & 1.4816 & -3.3923 & 2.3443 & 0.9096 & -2.0487 & 1.5311 & 0.6066 & -1.3524 & 1.0451 & 0.4164 & -0.9239 \\
\hline 0.1361 & 4.2882 & 1.4868 & -3.5075 & 2.3406 & 0.9117 & -2.1136 & 1.5286 & 0.6080 & -1.3944 & 1.0448 & 0.4174 & -0.9524 \\
\hline 0.1747 & 4.2806 & 1.4912 & -3.6553 & 2.3356 & 0.9133 & -2.1966 & 1.5254 & 0.6090 & -1.4483 & 1.0446 & 181 & -0.9890 \\
\hline 0.2098 & 4.2712 & 1.4986 & -3.8403 & 2.3299 & 0.9163 & -2.3003 & 1.5217 & 0.6111 & -1.5155 & 1.0442 & 0.4195 & -1.0348 \\
\hline 0.2424 & 4.2604 & 1.5049 & -4.0557 & 2.3228 & 0.9186 & -2.4209 & 1.5166 & 0.6126 & -1.5937 & 1.0438 & 0.4207 & -1.0880 \\
\hline 0.2722 & 4.2473 & 1.5142 & -4.3092 & 2.3146 & 0.9224 & -2.5623 & 1.5115 & 0.6152 & -1.6854 & 1.0432 & 0.4225 & -1.1504 \\
\hline 0.2998 & 4.2328 & 1.5214 & -4.5966 & 2.3054 & 0.9251 & -2.7222 & 1.5051 & 0.6172 & -1.7892 & 1.0427 & 0.4239 & -1.2211 \\
\hline 0.3250 & 4.2159 & 1.5316 & -4.9295 & 2.2950 & 0.9294 & -2.9069 & 1.4988 & 0.6201 & -1.9092 & 1.0421 & 0.4261 & -1.3027 \\
\hline 0.3484 & 4.1970 & 1.5393 & -5.3061 & 2.2836 & 0.9323 & -3.1153 & 1.4910 & 0.6223 & -2.0446 & 1.0414 & 0.4276 & -1.3949 \\
\hline 0.3698 & 4.1754 & 1.5506 & -5.7456 & 2.2706 & 0.9372 & -3.3576 & 1.4820 & 0.6258 & -2.2021 & 1.0407 & 0.4301 & -1.5022 \\
\hline 0.3896 & 4.1512 & 1.5570 & -6.2517 & 2.2557 & 0.9395 & -3.6360 & 1.4729 & 0.6276 & -2.3830 & 1.0399 & 0.4315 & -1.6255 \\
\hline 0.4076 & 4.1231 & 1.5684 & -6.8571 & 2.2390 & 0.9445 & -3.9678 & 1.4618 & 0.6314 & -2.5990 & 1.0390 & 0.4341 & -1.7727 \\
\hline 0.4244 & 4.0913 & 1.5723 & -7.5867 & 2.2201 & 0.9456 & -4.3666 & 1.4492 & 0.6325 & -2.8586 & 1.0382 & 0.4350 & -1.9496 \\
\hline 0.4397 & 4.0530 & 1.5830 & -8.4966 & 2.1974 & 0.9506 & -4.8625 & 1.4346 & 0.6363 & -3.1816 & 1.0372 & 0.4377 & -2.1698 \\
\hline 0.4539 & 4.0080 & 1.5835 & -9.7325 & 2.1711 & 0.9501 & -5.5341 & 1.4177 & 0.6366 & -3.6193 & 1.0362 & 0.4380 & -2.4681 \\
\hline 0.4668 & 3.9493 & 1.5867 & -11.3597 & 2.1372 & 0.9517 & -6.4165 & 1.3952 & 0.6383 & -4.1946 & 1.0352 & 0.4392 & -2.8604 \\
\hline 0.4789 & 3.8739 & 1.5552 & -14.2570 & 2.0940 & 0.9350 & -7.9835 & 1.3672 & 0.6282 & -5.2170 & 1.0341 & 0.4324 & -3.5575 \\
\hline 0.4898 & 3.7327 & 1.5577 & -21.1665 & 2.0150 & 0.9391 & -11.7109 & 1.3157 & 0.6317 & -7.6503 & 1.0329 & 0.4349 & -5.2166 \\
\hline 0.5000 & 3.2361 & -0.2671 & -70.2157 & 1.7430 & -0.0257 & -38.1310 & 1.1383 & 0.0021 & -24.9039 & 1.0317 & 0.0054 & -16.9815 \\
\hline
\end{tabular}


Table 3.20 Normalized $K_{\mathrm{l}}, T_{11}$ and $T_{33}$ for $\mathrm{a} / \mathrm{W}=0.6, \mathrm{t} / \mathrm{W}=0.5, \mathrm{SECP}$

\begin{tabular}{|c|c|c|c|c|c|c|c|c|c|c|c|c|}
\hline \multirow{2}{*}{$z / t$} & \multicolumn{3}{|c|}{ Uniform } & \multicolumn{3}{|c|}{ Linear } & \multicolumn{3}{|c|}{ Parabolic } & \multicolumn{3}{|c|}{ Cubic } \\
\hline & $K_{I}$ & $T_{11}$ & $T_{33}$ & $K_{I}$ & $T_{11}$ & $T_{33}$ & $K_{I}$ & $T_{I I}$ & $T_{33}$ & $K_{I}$ & $T_{l l}$ & $T_{33}$ \\
\hline 0.0000 & 4.2664 & 1.4081 & -1.3548 & 2.3356 & 0.8846 & -0.8512 & 1.5267 & 0.5946 & -0.5708 & 1.0410 & 0.4092 & -0.3923 \\
\hline 0.0615 & 4.2640 & 1.4050 & -1.3855 & 2.3340 & 0.8823 & -0.8697 & 1.5254 & 0.5930 & -0.5829 & 1.0403 & 0.4081 & -0.4005 \\
\hline 0.1161 & 4.2579 & 1.3987 & -1.4663 & 2.3299 & 0.8774 & -0.9180 & 1.5229 & 0.5894 & -0.6146 & 1.0383 & 0.4056 & -0.4221 \\
\hline 0.1647 & 4.2486 & 1.3896 & -1.5863 & 2.3237 & 0.8702 & -0.9895 & 1.5185 & 0.5842 & -0.6614 & 1.0354 & 0.4019 & -0.4539 \\
\hline 0.2078 & 4.2364 & 1.3788 & -1.7391 & 2.3154 & 0.8616 & -1.0800 & 1.5128 & 0.5779 & -0.7205 & 1.0315 & 0.3975 & -0.4941 \\
\hline 0.2462 & 4.2216 & 1.3667 & -1.9209 & 2.3054 & 0.8517 & -1.1870 & 1.5058 & 0.5708 & -0.7903 & 1.0269 & 0.3924 & -0.5416 \\
\hline 0.2802 & 4.2041 & 1.3541 & -2.1299 & 2.2937 & 0.8412 & -1.3090 & 1.4981 & 0.5633 & -0.8697 & 1.0214 & 0.3872 & -0.5955 \\
\hline 0.3105 & 4.1839 & 1.3410 & -2.3659 & 2.2807 & 0.8302 & -1.4458 & 1.4891 & 0.5554 & -0.9586 & 1.0152 & 0.3817 & -0.6559 \\
\hline 0.3374 & 4.1613 & 1.3279 & -2.6301 & 2.2659 & 0.8190 & -1.5976 & 1.4794 & 0.5475 & -1.0571 & 1.0084 & 0.3762 & -0.7228 \\
\hline 0.3613 & 4.1361 & 1.3149 & -2.9254 & 2.2493 & 0.8078 & -1.7658 & 1.4683 & 0.5397 & -1.1661 & 1.0007 & 0.3708 & -0.7968 \\
\hline 0.3825 & 4.1079 & 1.3021 & -3.2564 & 2.2313 & 0.7966 & -1.9528 & 1.4558 & 0.5321 & -1.2872 & 0.9924 & 0.3656 & -0.8791 \\
\hline 0.4014 & 4.0771 & 1.2895 & -3.6304 & 2.2114 & 0.7857 & -2.1624 & 1.4426 & 0.5247 & -1.4229 & 0.9833 & 0.3606 & -0.9713 \\
\hline 0.4182 & 4.0428 & 1.2771 & -4.0582 & 2.1896 & 0.7749 & -2.4003 & 1.4285 & 0.5176 & -1.5770 & 0.9734 & 0.3559 & -1.0761 \\
\hline 0.4331 & 4.0051 & 1.2646 & -4.5565 & 2.1657 & 0.7644 & -2.6754 & 1.4123 & 0.5109 & -1.7551 & 0.9626 & 0.3514 & -1.1972 \\
\hline 0.4463 & 3.9627 & 1.2520 & -5.1517 & 2.1394 & 0.7541 & -3.0016 & 1.3952 & 0.5045 & -1.9665 & 0.9507 & 0.3472 & -1.3411 \\
\hline 0.4581 & 3.9148 & 1.2388 & -5.8885 & 2.1100 & 0.7437 & -3.4029 & 1.3757 & 0.4982 & -2.2268 & 0.9375 & 0.3431 & -1.5182 \\
\hline 0.4686 & 3.8592 & 1.2261 & -6.8499 & 2.0765 & 0.7341 & -3.9234 & 1.3535 & 0.4927 & -2.5647 & 0.9224 & 0.3395 & -1.7484 \\
\hline 0.4779 & 3.7919 & 1.2130 & -8.2124 & 2.0363 & 0.7250 & -4.6573 & 1.3272 & 0.4877 & -3.0415 & 0.9044 & 0.3363 & -2.0731 \\
\hline 0.4861 & 3.7048 & 1.1931 & -10.3862 & 1.9852 & 0.7135 & -5.8229 & 1.2938 & 0.4814 & -3.7997 & 0.8817 & 0.3322 & -2.5897 \\
\hline 0.4935 & 3.5749 & 0.8488 & -14.0204 & 1.9107 & 0.5316 & -7.7663 & 1.2452 & 0.3644 & -5.0646 & 0.8485 & 0.2527 & -3.4515 \\
\hline 0.5000 & 3.0268 & -0.1890 & -62.2295 & 1.6112 & 0.0054 & -33.4261 & 1.0500 & 0.0236 & -21.7862 & 0.7155 & 0.0207 & -14.8468 \\
\hline
\end{tabular}


Table 3.21 Normalized $K_{\mathrm{l}}, T_{11}$ and $T_{33}$ for $\mathrm{a} / \mathrm{W}=0.6, \mathrm{t} / \mathrm{W}=1.0, \mathrm{SECP}$

\begin{tabular}{|c|c|c|c|c|c|c|c|c|c|c|c|c|}
\hline \multirow{2}{*}{$z / t$} & \multicolumn{3}{|c|}{ Uniform } & \multicolumn{3}{|c|}{ Linear } & \multicolumn{3}{|c|}{ Parabolic } & \multicolumn{3}{|c|}{ Cubic } \\
\hline & $K_{I}$ & $T_{11}$ & $T_{33}$ & $K_{l}$ & $T_{11}$ & $T_{33}$ & $K_{I}$ & $T_{l 1}$ & $T_{33}$ & $K_{I}$ & $T_{11}$ & $T_{33}$ \\
\hline 0.0000 & 4.1967 & 1.2844 & -0.2317 & 2.2966 & 0.8137 & -0.1523 & 1.5013 & 0.5491 & -0.1038 & 1.0241 & 0.3786 & -0.0718 \\
\hline 0.0615 & 4.1956 & 1.2845 & -0.2571 & 2.2958 & 0.8135 & -0.1682 & 1.5007 & 0.5490 & -0.1145 & 1.0237 & 0.3785 & -0.0792 \\
\hline 0.1161 & 4.1919 & 1.2853 & -0.3237 & 2.2933 & 0.8135 & -0.2099 & 1.4994 & 0.5488 & -0.1424 & 1.0225 & 0.3783 & -0.0984 \\
\hline 0.1647 & 4.1862 & 1.2846 & -0.4229 & 2.2891 & 0.8123 & -0.2718 & 1.4962 & 0.5477 & -0.1839 & 1.0206 & 0.3775 & -0.1268 \\
\hline 0.2078 & 4.1779 & 1.2812 & -0.5494 & 2.2836 & 0.8091 & -0.3505 & 1.4923 & 0.5452 & -0.2364 & 1.0179 & 0.3757 & -0.1629 \\
\hline 0.2462 & 4.1673 & 1.2740 & -0.6999 & 2.2765 & 0.8031 & -0.4437 & 1.4878 & 0.5408 & -0.2985 & 1.0145 & 0.3725 & -0.2054 \\
\hline 0.2802 & 4.1542 & 1.2630 & -0.8724 & 2.2676 & 0.7945 & -0.5498 & 1.4813 & 0.5345 & -0.3690 & 1.0102 & 0.3680 & -0.2536 \\
\hline 0.3105 & 4.1380 & 1.2480 & -1.0661 & 2.2566 & 0.7830 & -0.6680 & 1.4742 & 0.5261 & -0.4472 & 1.0050 & 0.3621 & -0.3071 \\
\hline 0.3374 & 4.1186 & 1.2294 & -1.2810 & 2.2437 & 0.7688 & -0.7980 & 1.4650 & 0.5159 & -0.5328 & 0.9988 & 0.3549 & -0.3655 \\
\hline 0.3613 & 4.0962 & 1.2078 & -1.5184 & 2.2287 & 0.7523 & -0.9400 & 1.4552 & 0.5041 & -0.6261 & 0.9917 & 0.3466 & -0.4291 \\
\hline 0.3825 & 4.0701 & 1.1836 & -1.7812 & 2.2114 & 0.7338 & -1.0954 & 1.4432 & 0.4910 & -0.7277 & 0.9836 & 0.3373 & -0.4982 \\
\hline 0.4014 & 4.0404 & 1.1575 & -2.0738 & 2.1918 & 0.7137 & -1.2662 & 1.4299 & 0.4768 & -0.8390 & 0.9744 & 0.3275 & -0.5738 \\
\hline 0.4182 & 4.0068 & 1.1299 & -2.4037 & 2.1697 & 0.6925 & -1.4563 & 1.4150 & 0.4619 & -0.9624 & 0.9640 & 0.3172 & -0.6577 \\
\hline 0.4331 & 3.9685 & 1.1013 & -2.7823 & 2.1448 & 0.6704 & -1.6715 & 1.3979 & 0.4467 & -1.1018 & 0.9524 & 0.3067 & -0.7524 \\
\hline 0.4463 & 3.9249 & 1.0721 & -3.2279 & 2.1164 & 0.6479 & -1.9216 & 1.3790 & 0.4314 & -1.2636 & 0.9395 & 0.2962 & -0.8622 \\
\hline 0.4581 & 3.8749 & 1.0421 & -3.7706 & 2.0844 & 0.6249 & -2.2223 & 1.3576 & 0.4161 & -1.4579 & 0.9248 & 0.2858 & -0.9943 \\
\hline 0.4686 & 3.8164 & 1.0115 & -4.4620 & 2.0476 & 0.6019 & -2.6009 & 1.3331 & 0.4012 & -1.7026 & 0.9080 & 0.2758 & -1.1606 \\
\hline 0.4779 & 3.7455 & 0.9744 & -5.3969 & 2.0035 & 0.5759 & -3.1075 & 1.3039 & 0.3847 & -2.0302 & 0.8881 & 0.2647 & -1.3834 \\
\hline 0.4861 & 3.6536 & 0.9043 & -6.7583 & 1.9476 & 0.5335 & -3.8388 & 1.2671 & 0.3582 & -2.5036 & 0.8630 & 0.2470 & -1.7055 \\
\hline 0.4935 & 3.5169 & 0.5522 & -8.8395 & 1.8670 & 0.3447 & -4.9499 & 1.2143 & 0.2373 & -3.2236 & 0.8269 & 0.1650 & -2.1955 \\
\hline 0.5000 & 2.9701 & 0.3062 & -47.9023 & 1.5652 & 0.2393 & -25.5402 & 1.0178 & 0.1728 & -16.6102 & 0.6931 & 0.1216 & -11.3112 \\
\hline
\end{tabular}


Table 3.22 Normalized $K_{\mathrm{I}}, T_{11}$ and $T_{33}$ for $\mathrm{a} / \mathrm{W}=0.6, \mathrm{t} / \mathrm{W}=2.0, \mathrm{SECP}$

\begin{tabular}{|c|c|c|c|c|c|c|c|c|c|c|c|c|}
\hline \multirow{2}{*}{$2 / t$} & \multicolumn{3}{|c|}{ Uniform } & \multicolumn{3}{|c|}{ Linear } & \multicolumn{3}{|c|}{ Parabolic } & \multicolumn{3}{|c|}{ Cubic } \\
\hline & $K_{I}$ & $T_{u}$ & $T_{33}$ & $K_{I}$ & $T_{l l}$ & $T_{33}$ & $K_{I}$ & $T_{I I}$ & $T_{33}$ & $K_{I}$ & $T_{l l}$ & $T_{33}$ \\
\hline 0.0000 & 4.1163 & 1.0737 & 0.2522 & 2.2472 & 0.6794 & 0.1578 & 1.4683 & 0.4584 & 0.1061 & 1.0015 & 0.3160 & 0.0930 \\
\hline 0.0615 & 4.1165 & 1.0783 & 0.2429 & 2.2472 & 0.6824 & 0.1518 & 1.4690 & 0.4604 & 0.1020 & 1.0015 & 0.3174 & 0.0933 \\
\hline 0.1161 & 4.1170 & 1.0908 & 0.2172 & 2.2476 & 0.6904 & 0.1352 & 1.4690 & 0.4659 & 0.0907 & 1.0016 & 0.3212 & 0.0939 \\
\hline 0.1647 & 4.1172 & 1.1089 & 0.1747 & 2.2476 & 0.7020 & 0.1079 & 1.4690 & 0.4737 & 0.0722 & 1.0016 & 0.3266 & 0.0943 \\
\hline 0.2078 & 4.1168 & 1.1305 & 0.1139 & 2.2472 & 0.7156 & 0.0689 & 1.4683 & 0.4829 & 0.0458 & 1.0014 & 0.3330 & 0.0938 \\
\hline 0.2462 & 4.1154 & 1.1530 & 0.0329 & 2.2459 & 0.7297 & 0.0173 & 1.4677 & 0.4923 & 0.0110 & 1.0009 & 0.3394 & 0.0911 \\
\hline 0.2802 & 4.1123 & 1.1744 & -0.0699 & 2.2437 & 0.7428 & -0.0479 & 1.4663 & 0.5010 & -0.0330 & 0.9998 & 0.3454 & 0.0851 \\
\hline 0.3105 & 4.1072 & 1.1918 & -0.1958 & 2.2403 & 0.7532 & -0.1274 & 1.4637 & 0.5079 & -0.0864 & 0.9981 & 0.3500 & 0.0741 \\
\hline 0.3374 & 4.0997 & 1.2038 & -0.3457 & 2.2352 & 0.7598 & -0.2215 & 1.4598 & 120 & & 0.9955 & 528 & 0.0567 \\
\hline 0.3613 & 4.0889 & 1.2088 & -0.5206 & 2.2278 & 0.7618 & -0.3306 & 1.4552 & 0.5130 & -0.2225 & 0.9920 & 0.3533 & 0.0316 \\
\hline 0.3825 & 4.0745 & 1.2064 & -0.7219 & 2.2179 & 0.7586 & -0.4553 & 1.4479 & 0.5103 & -0.3056 & 0.9873 & 0.3513 & -0.0024 \\
\hline 0.4014 & 4.0559 & 1.1963 & -0.9517 & 2.2053 & 0.7500 & -0.5962 & 1.4392 & 0.5039 & -0.3991 & 0.9813 & 0.3467 & -0.0460 \\
\hline 0.4182 & 4.0324 & 1.1790 & -1.2138 & 2.1896 & 0.7362 & -0.7551 & 1.4285 & 0.4938 & -0.5039 & 0.9737 & 0.3396 & -0.1000 \\
\hline 0.4331 & 4.0029 & 1.1549 & -1.5142 & 2.1697 & 0.7175 & -0.9348 & 1.4150 & 0.4803 & -0.6218 & 0.9645 & 0.3301 & -0.1651 \\
\hline 0.4463 & 3.9670 & 1.1246 & -1.8630 & 2.1461 & 0.6941 & -1.1402 & 1.3986 & 0.4637 & -0.7559 & 0.9532 & 0.3184 & -0.2423 \\
\hline 0.4581 & 3.9229 & 1.0882 & -2.2775 & 2.1168 & 0.6662 & -1.3800 & 1.3792 & 0.4442 & -0.9117 & 0.9395 & 0.3049 & -0.3333 \\
\hline 0.4686 & 3.8682 & 1.0457 & -2.7875 & 2.0811 & 0.6341 & -1.6700 & 1.3551 & 0.4221 & -1.0993 & 0.9230 & 0.2897 & -0.4421 \\
\hline 0.4779 & 3.7988 & 0.9917 & -3.4514 & 2.0363 & 0.5952 & -2.0406 & 1.3251 & 0.3961 & -1.3385 & 0.9024 & 0.2720 & -0.5773 \\
\hline 0.4861 & 3.7053 & 0.9088 & -4.3953 & 1.9774 & 0.5407 & -2.5583 & 1.2860 & 0.3608 & -1.6724 & 0.8756 & 0.2481 & -0.7606 \\
\hline 0.4935 & 3.5647 & 0.6467 & -5.8930 & 1.8910 & 0.3931 & -3.3675 & 1.2290 & 0.2663 & -2.1945 & 0.8367 & 0.1843 & -1.0447 \\
\hline 0.5000 & 2.9943 & 0.5747 & -36.4877 & 1.5707 & 0.3710 & -19.4322 & 1.0202 & 0.2577 & -12.6242 & 0.6945 & 0.1793 & -6.5329 \\
\hline
\end{tabular}


Table 3.23 Normalized $K_{\mathrm{I}}, T_{11}$ and $T_{33}$ for $\mathrm{a} / \mathrm{W}=0.6, \mathrm{t} / \mathrm{W}=4.0, \mathrm{SECP}$

\begin{tabular}{|c|c|c|c|c|c|c|c|c|c|c|c|c|}
\hline \multirow{2}{*}{$z / t$} & \multicolumn{3}{|c|}{ Uniform } & \multicolumn{3}{|c|}{ Linear } & \multicolumn{3}{|c|}{ Parabolic } & \multicolumn{3}{|c|}{ Cubic } \\
\hline & $K_{I}$ & $T_{11}$ & $T_{33}$ & $K_{I}$ & $T_{11}$ & $T_{33}$ & $K_{I}$ & $T_{I I}$ & $T_{33}$ & $K_{I}$ & $T_{11}$ & $T_{33}$ \\
\hline 0.0000 & 4.0679 & 1.0383 & 0.3063 & 2.2166 & 0.6561 & 0.1932 & 1.4479 & 0.4425 & 0.1302 & 0.9874 & 0.3050 & 0.0897 \\
\hline 0.0615 & 4.0684 & 1.0381 & 0.3058 & 2.2170 & 0.6560 & 0.1928 & 1.4486 & 0.4424 & 0.1300 & 0.9875 & 0.3050 & 0.0896 \\
\hline 0.1161 & 4.0691 & 1.0383 & 0.3041 & 2.2175 & 0.6561 & 0.1918 & 1.4486 & 0.4425 & 0.1292 & 0.9878 & 0.3050 & 0.0891 \\
\hline 0.1647 & 4.0703 & 1.0396 & 0.3007 & 2.2183 & 0.6570 & 0.1895 & 1.4492 & 0.4431 & 0.1277 & 0.9881 & 0.3055 & 0.0880 \\
\hline 0.2078 & 4.0720 & 1.0434 & 0.2940 & 2.2192 & 0.6595 & 0.1850 & 1.4499 & 0.4449 & 0.1246 & 0.9886 & 0.3067 & 0.0859 \\
\hline 0.2462 & 4.0738 & 1.0508 & 0.2814 & 2.2205 & 0.6644 & 0.1768 & 1.4505 & 0.4482 & 0.1190 & 0.9891 & 0.3090 & 0.0820 \\
\hline 0.2802 & 4.0760 & 1.0634 & 0.2597 & 2.2218 & 0.6727 & 0.1627 & 1.4512 & 0.4538 & 0.1094 & 0.9897 & 0.3129 & 0.0753 \\
\hline 0.3105 & 4.0776 & 1.0812 & 0.2245 & 2.2227 & 0.6842 & 0.1398 & 1.4519 & 0.4617 & 0.0939 & 0.9901 & 0.3183 & 0.0646 \\
\hline 0.3374 & 4.0786 & 1.1040 & 711 & 2.2231 & 0.6989 & 53 & 1.4525 & 0.4716 & 05 & 0.9904 & 0.3252 & 0.0484 \\
\hline 0.3613 & 4.0783 & 1.1300 & 0.0944 & 2.2231 & 0.7153 & 0.0561 & 1.4519 & 0.4827 & 0.0372 & 0.9902 & 0.3328 & 0.0254 \\
\hline 0.3825 & 4.0762 & 1.1563 & -0.0104 & 2.2214 & 0.7316 & -0.0107 & 1.4512 & 0.4936 & -0.0080 & 0.9893 & 0.3403 & -0.0057 \\
\hline 0.4014 & 4.0712 & 1.1792 & -0.1478 & 2.2179 & 0.7455 & -0.0978 & 1.4486 & 0.5027 & -0.0666 & 0.9876 & 0.3465 & -0.0461 \\
\hline 0.4182 & 4.0625 & 1.1950 & -0.3221 & 2.2118 & 0.7545 & -0.2077 & 1.4446 & 0.5085 & -0.1404 & 0.9847 & 0.3504 & -0.0968 \\
\hline 0.4331 & 4.0488 & 1.2006 & -0.5383 & 2.2023 & 0.7564 & -0.3430 & 1.4379 & 0.5093 & -0.2309 & 0.9802 & 0.3508 & -0.1589 \\
\hline 0.4463 & 4.0283 & 1.1935 & -0.8030 & 2.1882 & 0.7496 & -0.5071 & 1.4285 & 0.5040 & -0.3402 & 0.9735 & 0.3469 & -0.2337 \\
\hline 0.4581 & 3.9989 & 1.1722 & -1.1274 & 2.1684 & 0.7327 & -0.7056 & 1.4143 & 0.4916 & -0.4714 & 0.9640 & 0.3381 & -0.3234 \\
\hline 0.4686 & 3.9571 & 1.1362 & -1.5307 & 2.1408 & 0.7051 & -0.9481 & 1.3952 & 0.4718 & -0.6304 & 0.9508 & 0.3242 & -0.4317 \\
\hline 0.4779 & 3.8979 & 1.0812 & -2.0538 & 2.1013 & 0.6641 & -1.2555 & 1.3687 & 0.4431 & -0.8305 & 0.9323 & 0.3042 & -0.5677 \\
\hline 0.4861 & 3.8104 & 0.9967 & -2.7894 & 2.0439 & 0.6042 & -1.6762 & 1.3301 & 0.4023 & -1.1025 & 0.9058 & 0.2762 & -0.7522 \\
\hline 0.4935 & 3.6680 & 0.7817 & -3.9509 & 1.9535 & 0.4725 & -2.3230 & 1.2696 & 0.3165 & -1.5187 & 0.8643 & 0.2180 & -1.0346 \\
\hline 0.5000 & 3.0543 & 0.7232 & -27.6244 & 1.5998 & 0.4424 & -14.7586 & 1.0389 & 0.3036 & -9.5845 & 0.7071 & 0.2105 & -6.5235 \\
\hline
\end{tabular}

61 
Table 3.24 Normalized $K_{\mathrm{l}}, T_{11}$ and $T_{33}$ for $\mathrm{a} / \mathrm{W}=0.8, \mathrm{t} / \mathrm{W}=0.1, \mathrm{SECP}$

\begin{tabular}{|c|c|c|c|c|c|c|c|c|c|c|c|c|}
\hline \multirow{2}{*}{$z / t$} & \multicolumn{3}{|c|}{ Uniform } & \multicolumn{3}{|c|}{ Linear } & \multicolumn{3}{|c|}{ Parabolic } & \multicolumn{3}{|c|}{ Cubic } \\
\hline & $\boldsymbol{K}_{I}$ & $T_{\mu}$ & $T_{33}$ & $K_{I}$ & $T_{l I}$ & $T_{33}$ & $\boldsymbol{K}_{I}$ & $T_{l 1}$ & $T_{33}$ & $K_{I}$ & $T_{11}$ & $T_{33}$ \\
\hline 0.0000 & 12.7838 & 9.2375 & -17.3030 & 7.7363 & 5.9242 & -10.6747 & 5.4367 & 4.2416 & -7.4972 & 4.0728 & 3.2081 & -5.6131 \\
\hline 0.0346 & 12.7781 & 9.2337 & -17.3698 & 7.7344 & 5.9218 & -10.7154 & 5.4354 & 4.2399 & -7.5258 & 4.0728 & 3.2068 & -5.6346 \\
\hline 0.0679 & 12.7725 & 9.2393 & -17.5600 & 7.7307 & 5.9250 & -10.8312 & 5.4327 & 4.2422 & -7.6072 & 4.0710 & 3.2085 & -5.6956 \\
\hline 0.1001 & 12.7612 & 9.2481 & -17.8666 & 7.7242 & 5.9298 & -11.0181 & 5.4274 & 4.2457 & -7.7385 & 4.0675 & 3.2111 & -5.7940 \\
\hline 0.1311 & 12.7499 & 9.2598 & -18.2871 & 7.7149 & 5.9364 & -11.2742 & 5.4221 & 4.2503 & -7.9186 & 4.0621 & 3.2145 & -5.9289 \\
\hline 0.1610 & 12.7330 & 9.2747 & -18.8220 & 7.7027 & 5.9448 & -11.5999 & 5.4128 & 4.2561 & -8.1475 & 4.0568 & 3.2189 & -6.1005 \\
\hline 0.1898 & 12.7103 & 9.2925 & -19.4756 & 7.6887 & 5.9548 & -11.9978 & 5.4035 & 4.2632 & -8.4271 & 4.0479 & 3.2241 & -6.3101 \\
\hline 0.2176 & 12.6819 & 9.3133 & -20.2561 & 18 & 666 & 27 & 15 & 114 & 609 & 4.0390 & 3.2303 & -6.5603 \\
\hline 0.2444 & 12.6478 & 9.3 & -21.1765 & 7. & 04 & 325 & 5.3767 & 4.2811 & -9.1543 & 4.0283 & 3.2375 & -6.8552 \\
\hline 0.2702 & 12.6136 & 9.3643 & -22.2561 & 7.6284 & 5.9956 & 5889 & 5.3606 & 4.2918 & -9.6156 & 4.0176 & 3.2455 & -7.2009 \\
\hline 0.2951 & 12.5678 & 9.3951 & -23.5224 & 7.6020 & 6.0130 & -14.4583 & 5.3418 & 4.3041 & -10.1563 & 4.0032 & 3.2547 & -7.6062 \\
\hline 0.3191 & 12.5161 & 9.4297 & -25.0156 & 7.5706 & 6.0329 & -15.3651 & 5.3201 & 4.3180 & -10.7936 & 3.9851 & 3.2652 & -8.0837 \\
\hline 0.3423 & 12.4584 & 9.4689 & -26.7948 & 7.5334 & 6.0553 & -16.4448 & 5.2944 & 4.3339 & -11.5524 & 3.9670 & 3.2770 & -8.6524 \\
\hline 0.3646 & 12.3888 & 9.5137 & -28.9484 & 7.4902 & 6.0813 & -17.7509 & 5.2643 & 4.3521 & -12.4702 & 3.9433 & 3.2907 & -9.3401 \\
\hline 0.3861 & 12.3071 & 9.5648 & -31.6098 & 7.4400 & 6.1109 & -19.3638 & 5.2286 & 4.3731 & -13.6036 & 3.9177 & 3.3063 & -10.1895 \\
\hline 0.4068 & 12.2071 & 9.6182 & -34.9826 & 7.3788 & 6.1419 & -21.4064 & 5.1843 & 4.3949 & -15.0389 & 3.8844 & 3.3226 & -11.2650 \\
\hline 0.4269 & 12.0885 & 9.6433 & -39.3511 & 7.3032 & 6.1559 & -24.0501 & 5.1312 & 4.4048 & -16.8966 & 3.8453 & 3.3301 & -12.6570 \\
\hline 0.4461 & 11.9277 & 9.4959 & -45.0560 & 7.2058 & 6.0662 & -27.5009 & 5.0634 & 4.3420 & -19.3213 & 3.7934 & 3.2828 & -14.4738 \\
\hline 0.4647 & 11.7162 & 8.3980 & -51.8837 & 7.0757 & 5.4033 & -31.6299 & 4.9715 & 3.8763 & -22.2226 & 3.7248 & 2.9340 & -16.6477 \\
\hline 0.4827 & 11.4241 & 2.1455 & -55.3031 & 6.8972 & 1.6315 & -33.7032 & 4.8452 & 1.2266 & -23.6795 & 3.6305 & 0.9488 & -17.7395 \\
\hline 0.5000 & 9.9582 & 5.0786 & -231.4927 & 6.0072 & 3.4217 & -139.9995 & 4.2205 & 2.4857 & -98.3637 & 3.1623 & 1.8925 & -73.6913 \\
\hline
\end{tabular}

62 
Table 3.25 Normalized $K_{\mathrm{I}}, T_{11}$ and $T_{33}$ for $\mathrm{a} / \mathrm{W}=0.8, \mathrm{t} / \mathrm{W}=0.2$, SECP

\begin{tabular}{|c|c|c|c|c|c|c|c|c|c|c|c|c|}
\hline \multirow{2}{*}{$z / t$} & \multicolumn{3}{|c|}{ Uniform } & \multicolumn{3}{|c|}{ Linear } & \multicolumn{3}{|c|}{ Parabolic } & \multicolumn{3}{|c|}{ Cubic } \\
\hline & $K_{I}$ & $T_{11}$ & $T_{33}$ & $K_{I}$ & $T_{11}$ & $T_{33}$ & $K_{I}$ & $T_{l l}$ & $T_{33}$ & $K_{I}$ & $T_{11}$ & $T_{33}$ \\
\hline 0.0000 & 12.7556 & 9.0117 & -9.5737 & 7.7233 & 5.8009 & -5.9337 & 5.4274 & 4.1559 & -4.1637 & 4.0675 & 3.1438 & -3.1139 \\
\hline 0.0490 & 12.7499 & 9.0040 & -9.6904 & 7.7186 & 5.7958 & -6.0053 & 5.4248 & 4.1523 & -4.2140 & 4.0657 & 3.1411 & -3.1517 \\
\hline 0.0946 & 12.7330 & 8.9823 & -9.9725 & 7.7093 & 5.7816 & -6.1785 & 5.4181 & 4.1422 & -4.3358 & 4.0604 & 3.1335 & -3.2430 \\
\hline 0.1361 & 12.7103 & 8.9575 & -10.4257 & 7.6943 & 5.7648 & -6.4565 & 5.4075 & 4.1303 & -4.5312 & 4.0515 & 1246 & -3.3895 \\
\hline 0.1747 & 12.6762 & 8.9201 & -11.0026 & 7.6755 & 5.7400 & -6.8101 & 5.3941 & 4.1128 & -4.7798 & 4.0408 & 3.1115 & -3.5759 \\
\hline 0.2098 & 12.6421 & 8.8843 & -11.7185 & 7.6511 & 5.7158 & -7.2486 & 5.3767 & 4.0956 & -5.0881 & 4.0283 & 3.0986 & -3.8070 \\
\hline 0.2424 & 12.5964 & 8.8386 & -12.5450 & 7.6228 & 5.6854 & -7.7544 & 5.3566 & 4.0740 & -5.4436 & 4.0140 & 3.0825 & -4.0735 \\
\hline 0.2722 & 12.5448 & 8.7985 & -13.5062 & 7.5896 & 5.6581 & -8.3420 & 5.3337 & 4.0548 & -5.8566 & 3.9960 & 3.0680 & -4.3831 \\
\hline 0.2998 & 12.4872 & 8.7485 & -14.5850 & 7.5525 & 5.6249 & -9.0008 & 5.3079 & 4.0313 & -6.3196 & 3.9761 & 3.0504 & -4.7302 \\
\hline 0.3250 & 12.4178 & 8.7065 & -15.8190 & 7.5104 & 5.5963 & -9.7534 & 5.2780 & 4.0111 & -6.8486 & 3.9543 & 3.0352 & -5.1266 \\
\hline 0.3484 & 12.3421 & 8.6553 & -17.2000 & 7.4632 & 5.5621 & -10.5948 & 5.2451 & 3.9871 & -7.4398 & 3.9287 & 3.0172 & -5.5698 \\
\hline 0.3698 & 12.2601 & 8.6164 & -18.7901 & 7.4109 & 5.5354 & -11.5622 & 5.2065 & 3.9683 & -8.1196 & 3.9011 & 3.0030 & -6.0793 \\
\hline 0.3896 & 12.1657 & 8.5613 & -20.6011 & 7.3524 & 5.4990 & -12.6627 & 5.1662 & 3.9427 & -8.8929 & 3.8696 & 2.9838 & -6.6589 \\
\hline 0.4076 & 12.0586 & 8.5249 & -22.7396 & 7.2854 & 5.4741 & -13.9604 & 5.1186 & 3.9251 & -9.8047 & 3.8359 & 2.9707 & -7.3422 \\
\hline 0.4244 & 11.9403 & 8.4653 & -25.2874 & 7.2108 & 5.4353 & -15.5045 & 5.0662 & 3.8979 & -10.8895 & 3.7959 & 2.9503 & -8.1551 \\
\hline 0.4397 & 11.8001 & 8.4320 & -28.4290 & 7.1234 & 5.4126 & -17.4061 & 5.0047 & 3.8821 & -12.2255 & 3.7498 & 2.9384 & -9.1562 \\
\hline 0.4539 & 11.6378 & 8.3686 & -32.6452 & 7.0235 & 5.3721 & -19.9546 & 4.9336 & 3.8539 & -14.0158 & 3.6968 & 2.9172 & -10.4977 \\
\hline 0.4668 & 11.4329 & 8.3231 & -38.1498 & 6.8962 & 5.3432 & -23.2787 & 4.8452 & 3.8339 & -16.3509 & 3.6297 & 2.9021 & -12.2473 \\
\hline 0.4788 & 11.1754 & 8.1729 & -47.8469 & 6.7376 & 5.2521 & -29.1272 & 4.7339 & 3.7703 & -20.4593 & 3.5460 & 2.8544 & -15.3253 \\
\hline 0.4898 & 10.7206 & 8.1811 & -70.7431 & 6.4592 & 5.2605 & -42.9196 & 4.5380 & 3.7768 & -30.1476 & 3.3993 & 2.8593 & -22.5833 \\
\hline 0.5000 & 9.2249 & 2.4834 & -232.2721 & 5.5534 & 1.8605 & -140.1503 & 3.9011 & 1.3906 & -98.4450 & 2.9219 & 1.0723 & -73.7460 \\
\hline
\end{tabular}


Table 3.26 Normalized $K_{\mathrm{l}}, T_{11}$ and $T_{33}$ for $\mathrm{a} / \mathrm{W}=0.8, \mathrm{t} / \mathrm{W}=0.5, \mathrm{SECP}$

\begin{tabular}{|c|c|c|c|c|c|c|c|c|c|c|c|c|}
\hline \multirow{2}{*}{$z / t$} & \multicolumn{3}{|c|}{ Uniform } & \multicolumn{3}{|c|}{ Linear } & \multicolumn{3}{|c|}{ Parabolic } & \multicolumn{3}{|c|}{ Cubic } \\
\hline & $\boldsymbol{K}_{I}$ & $T_{11}$ & $T_{33}$ & $\overline{K_{I}}$ & $T_{l l}$ & $T T_{33}$ & $K_{I}$ & $T_{l I}$ & $T_{33}$ & $K_{I}$ & $T_{l 1}$ & $T_{33}$ \\
\hline 0.0000 & 12.5333 & 8.4329 & -1.2029 & 7.5934 & 5.4663 & -0.7330 & 5.3377 & 3.9250 & -0.5036 & 3.9996 & 2.9718 & -0.3712 \\
\hline 0.0615 & 12.5276 & 8.4301 & -1.3426 & 7.5887 & 5.4637 & -0.8201 & 5.3350 & 3.9229 & -0.5649 & 3.9978 & 2.9701 & -0.4172 \\
\hline 0.1161 & 12.5103 & 8.4258 & -1.7088 & 7.5782 & 5.4588 & -1.0484 & 5.3269 & 3.9188 & -0.7257 & 3.9924 & 2.9669 & -0.5376 \\
\hline 0.1647 & 12.4872 & 8.4090 & -2.2517 & 7.5611 & 5.4451 & -1.3866 & 5.3147 & 3.9084 & -0.9637 & 3.9833 & 2.9589 & -0.7158 \\
\hline 0.2078 & 12.4468 & 8.3732 & -2.9395 & 7.5382 & 5.4188 & -1.8145 & 5.2984 & 3.8890 & -1.2647 & 3.9707 & 2.9441 & -0.9414 \\
\hline 0.2462 & 12.4062 & 8.3131 & -3.7517 & 7.5094 & 5.3769 & -2.3190 & 5.2780 & 3.8585 & -1.6196 & 3.9543 & 2.9210 & -1.2073 \\
\hline 0.2802 & 12.3480 & 8.2293 & -4.6745 & 7.4739 & 5.3199 & -2.8910 & 5.2520 & 3.8174 & -2.0220 & 3.9360 & 2.8899 & -1.5088 \\
\hline 0.3105 & 12.2836 & 8.1210 & -5.7007 & 7.4323 & 5.2473 & -3.5259 & 5.2231 & 3.7654 & -2.4685 & 3.9140 & 2.8507 & -1.8434 \\
\hline 0.3374 & 12.2130 & 7.9916 & -6.8279 & 7.3837 & 5.1615 & 217 & 5.1885 & 042 & -2.9577 & 3.8863 & 2.8046 & -2.2100 \\
\hline 0.3613 & 12.1242 & 7.8442 & -8.0599 & 7.3278 & 5.0645 & -4.9803 & 5.1481 & 3.6351 & -3.4910 & 3.8565 & 2.7528 & -2.6096 \\
\hline 0.3825 & 12.0227 & 7.6827 & -9.4074 & 7.2646 & 4.9586 & -5.8079 & 5.1045 & 3.5601 & -4.0725 & 3.8227 & 2.6964 & -3.0454 \\
\hline 0.4014 & 11.9144 & 7.5103 & -10.8901 & 7.1938 & 4.8463 & -6.7160 & 5.0534 & 3.4805 & -4.7105 & 3.7860 & 2.6367 & -3.5235 \\
\hline 0.4182 & 11.7903 & 7.3309 & -12.5405 & 7.1153 & 4.7298 & -7.7238 & 4.9975 & 3.3981 & -5.4185 & 3.7441 & 2.5749 & -4.0540 \\
\hline 0.4331 & 11.6520 & 7.1472 & -14.4104 & 7.0276 & 4.6110 & -8.8626 & 4.9366 & 3.3143 & -6.2182 & 3.6976 & 2.5121 & -4.6533 \\
\hline 0.4463 & 11.4970 & 6.9620 & -16.5842 & 6.9296 & 4.4917 & -10.1827 & 4.8675 & 3.2303 & -7.1451 & 3.6456 & 2.4491 & -5.3478 \\
\hline 0.4581 & 11.3221 & 6.7768 & -19.2069 & 6.8195 & 4.3730 & -11.7710 & 4.7884 & 3.1468 & -8.2602 & 3.5872 & 2.3866 & -6.1832 \\
\hline 0.4686 & 11.1211 & 6.5981 & -22.5482 & 6.6937 & 4.2589 & -13.7892 & 4.7003 & 3.0668 & -9.6769 & 3.5203 & 2.3267 & -7.2445 \\
\hline 0.4779 & 10.8820 & 6.4272 & -27.1817 & 6.5445 & 4.1507 & -16.5810 & 4.5949 & 2.9912 & -11.6365 & 3.4414 & 2.2700 & -8.7124 \\
\hline 0.4861 & 10.5792 & 6.2501 & -34.4417 & 6.3557 & 4.0407 & -20.9462 & 4.4628 & 2.9146 & -14.7001 & 3.3420 & 2.2127 & -11.0071 \\
\hline 0.4935 & 10.1445 & 5.0527 & -46.4411 & 6.0882 & 3.3233 & -28.1511 & 4.2731 & 2.4121 & -19.7568 & 3.2006 & 1.8365 & -14.7943 \\
\hline 0.5000 & 8.4963 & 1.9925 & -202.8028 & 5.0889 & 1.5310 & -121.8192 & 3.5719 & 1.1585 & -85.4935 & 2.6749 & 0.8988 & -64.0222 \\
\hline
\end{tabular}

64 
Table 3.27 Normalized $K_{\mathrm{l}}, T_{11}$ and $T_{33}$ for $\mathrm{a} / \mathrm{W}=0.8, \mathrm{t} / \mathrm{W}=1.0, \mathrm{SECP}$

\begin{tabular}{|c|c|c|c|c|c|c|c|c|c|c|c|c|}
\hline \multirow{2}{*}{$z / t$} & \multicolumn{3}{|c|}{ Uniform } & \multicolumn{3}{|c|}{ Linear } & \multicolumn{3}{|c|}{ Parabolic } & \multicolumn{3}{|c|}{ Cubic } \\
\hline & $\bar{K}$ & $\bar{T}$ & $T T_{33}$ & $K_{l}$ & $T_{l l}$ & $T_{33}$ & $K_{I}$ & $T_{l l}$ & $T_{33}$ & $K_{I}$ & $T_{M}$ & $T_{33}$ \\
\hline 0.0000 & 12.2953 & 7.3713 & 1.5892 & 7.4478 & 4.8049 & 1.0305 & 5.2369 & 3.4609 & 0.7415 & 3.9250 & 2.6250 & 0.5622 \\
\hline 0.0615 & 12.2953 & 7.3909 & 1.5355 & 7.4468 & 4.8169 & 0.9963 & 5.2355 & 3.4693 & 0.7173 & 3.9232 & 2.6312 & 0.5441 \\
\hline 0.1161 & 12.2895 & 7.4453 & 1.3854 & 7.4439 & 4.8505 & 0.9010 & 5.2341 & 3.4926 & 0.6498 & 3.9213 & 2.6485 & 0.4935 \\
\hline 0.1647 & 12.2836 & 7.5241 & 1.1394 & 7.4391 & 4.8985 & 0.7451 & 5.2300 & 3.5258 & 0.5397 & 3.9195 & 2.6732 & 0.4109 \\
\hline 0.2078 & 12.2719 & 7.6177 & 0.7903 & 7.4303 & 4.9549 & 0.5247 & 5.2231 & 3.5648 & 0.3840 & 3.9140 & 2.7020 & 0.2941 \\
\hline 0.2462 & 12.2543 & 7.7130 & 0.3295 & 7.4187 & 5.0116 & 0.2345 & 5.2148 & 3.6036 & 0.1793 & 3.9066 & 2.7306 & 0.1407 \\
\hline 0.2802 & 12.2307 & 7.7986 & 491 & 7.4022 & 5.0613 & 287 & 5.2024 & 3.6373 & -0.0767 & 3.8992 & 2.7553 & -0.0511 \\
\hline 0.3105 & 12.2012 & 7.8600 & -0.9498 & 7.3807 & 5.0949 & -0.5674 & 5.1871 & 3.6595 & -0.3857 & 3.8863 & 2.7714 & -0.2826 \\
\hline 0.3374 & 12.1598 & 7.8878 & -1.7747 & 7.3533 & 5.1069 & -1.0824 & 5.1662 & 3.6663 & -0.7482 & 3.8714 & 2.7760 & -0.5541 \\
\hline 0.3613 & 12.1064 & 7.8749 & -2.7259 & 7.3180 & 5.0929 & -1.6747 & 5.1411 & 3.6549 & -1.1649 & 3.8509 & 2.7668 & -0.8663 \\
\hline 0.3825 & 12.0407 & 7.8181 & -3.8074 & 7.2745 & 5.0512 & -2.3462 & 5.1101 & 3.6239 & -1.6372 & 3.8284 & 2.7431 & -1.2200 \\
\hline 0.4014 & 11.9566 & 7.7181 & -5.0275 & 7.2218 & 4.9821 & -3.1014 & 5.0719 & 3.5737 & -2.1680 & 3.8000 & 2.7052 & -1.6176 \\
\hline 0.4182 & 11.8586 & 7.5775 & -6.4025 & 7.1577 & 4.8876 & -3.9496 & 5.0262 & 3.5059 & -2.7641 & 3.7650 & 2.6540 & -2.0641 \\
\hline 0.4331 & 11.7407 & 7.4009 & -7.9621 & 7.0818 & 4.7706 & -4.9084 & 4.9729 & 3.4225 & -3.4375 & 3.7242 & 2.5913 & -2.5686 \\
\hline 0.4463 & 11.6006 & 7.1937 & -9.7589 & 6.9917 & 4.6347 & -6.0087 & 4.9088 & 3.3261 & -4.2100 & 3.6761 & 2.5189 & -3.1472 \\
\hline 0.4581 & 11.4335 & 6.9603 & -11.8852 & 6.8847 & 4.4829 & -7.3054 & 4.8333 & 3.2188 & -5.1202 & 3.6192 & 2.4383 & -3.8290 \\
\hline 0.4686 & 11.2320 & 6.7064 & -14.5056 & 6.7569 & 4.3188 & -8.8969 & 4.7415 & 3.1031 & -6.2368 & 3.5511 & 2.3517 & -4.6653 \\
\hline 0.4779 & 10.9835 & 6.4175 & -17.9295 & 6.6004 & 4.1343 & -10.9678 & 4.6308 & 2.9735 & -7.6894 & 3.4677 & 2.2547 & -5.7532 \\
\hline 0.4861 & 10.6613 & 6.0143 & -22.7602 & 6.3975 & 3.8828 & -13.8789 & 4.4886 & 2.7975 & -9.7309 & 3.3603 & 2.1229 & -7.2819 \\
\hline 0.4935 & 10.1885 & 4.6873 & -29.9718 & 6.1036 & 3.0831 & -18.2125 & 4.2815 & 2.2379 & -12.7696 & 3.2048 & 1.7042 & -9.5571 \\
\hline 0.5000 & 8.4784 & 4.0152 & -158.9940 & 5.0634 & 2.7174 & -95.2762 & 3.5498 & 1.9873 & -66.8013 & 2.6571 & 1.5181 & -50.0011 \\
\hline
\end{tabular}

65 
Table 3.28 Normalized $K_{\mathrm{I}}, T_{11}$ and $T_{33}$ for a/W=0.8, $/ \mathrm{W}=2.0, \mathrm{SECP}$

\begin{tabular}{|c|c|c|c|c|c|c|c|c|c|c|c|c|}
\hline \multirow{2}{*}{$z / t$} & \multicolumn{3}{|c|}{ Uniform } & \multicolumn{3}{|c|}{ Linear } & \multicolumn{3}{|c|}{ Parabolic } & \multicolumn{3}{|c|}{ Cubic } \\
\hline & $K_{I}$ & $\bar{T}$ & $T_{33}$ & $K_{I}$ & $T_{l l}$ & $T_{33}$ & $K_{I}$ & $T_{11}$ & $T_{33}$ & $K_{I}$ & $T_{l l}$ & $T_{33}$ \\
\hline 0.0000 & 12.1242 & 7.0752 & 2.0187 & 7.3376 & 4.6081 & 1.3100 & 5.1579 & 3.3183 & 0.9415 & 3.8658 & 2.5166 & 0.7133 \\
\hline 0.0615 & 12.1242 & 7.0771 & 2.0121 & 7.3376 & 4.6095 & 1.3056 & 5.1579 & 3.3193 & 0.9384 & 3.8658 & 2.5174 & 0.7109 \\
\hline 0.1161 & 12.1242 & 7.0836 & 1.9932 & 7.3386 & 4.6141 & 1.2931 & 5.1579 & 3.3227 & 0.9293 & 3.8658 & 2.5200 & 0.7041 \\
\hline 0.1647 & 12.1242 & 7.0971 & 1.9589 & 7.3396 & 4.6233 & 1.2706 & 5.1592 & 3.3295 & 0.9132 & 3.8658 & 2.5252 & 0.6918 \\
\hline 0.2078 & 12.1242 & 7.1222 & 1.9020 & 7.3396 & 4.6399 & 1.2335 & 5.1592 & 3.3415 & 0.8866 & 3.8658 & 2.5344 & 0.6718 \\
\hline 0.2462 & 12.1242 & 7.1625 & 1.8096 & 7.3396 & 4.6661 & 1.1738 & 5.1592 & 3.3603 & 0.8440 & 3.8658 & 2.5484 & 0.6396 \\
\hline 0.2802 & 12.1242 & 7.2244 & 1.6644 & 7.3376 & 4.7055 & 1.0805 & 5.1579 & 3.3881 & 0.7775 & 3.8640 & 2.5692 & 0.5896 \\
\hline 0.3105 & 12.1183 & 7.3087 & 1.4428 & 7.3337 & 4.7581 & 0.9390 & 5.1551 & 3.4251 & 0.6772 & 3.8621 & 2.5968 & 0.5142 \\
\hline 0.3374 & 12.1123 & 7.4139 & 1.1 & 7.3268 & 4.8231 & 0.7335 & 5.1495 & 3.4703 & 0.5319 & 3.8584 & 2.6304 & 0.4051 \\
\hline 0.3613 & 12.0944 & 7.5309 & 0.6664 & 7.3160 & 4.8941 & 0.4476 & 5.1411 & 3.5193 & 0.3299 & 3.8528 & 2.6668 & 0.2537 \\
\hline 0.3825 & 12.0706 & 7.6439 & 0.0600 & 7.2992 & 4.9613 & 0.0663 & 5.1284 & 3.5653 & 0.0610 & 3.8415 & 2.7008 & 0.0521 \\
\hline 0.4014 & 12.0347 & 7.7331 & -0.7220 & 7.2755 & 5.0121 & -0.4237 & 5.1115 & 3.5995 & -0.2841 & 3.8284 & 2.7259 & -0.2063 \\
\hline 0.4182 & 11.9861 & 7.7774 & -1.6997 & 7.2418 & 5.0337 & -1.0340 & 5.0861 & 3.6129 & -0.7136 & 3.8095 & 2.7352 & -0.5280 \\
\hline 0.4331 & 11.9168 & 7.7595 & -2.8949 & 7.1958 & 5.0154 & -1.7775 & 5.0534 & 3.5981 & -1.2365 & 3.7850 & 2.7235 & -0.9196 \\
\hline 0.4463 & 11.8227 & 7.6675 & -4.3378 & 7.1345 & 4.9499 & -2.6719 & 5.0090 & 3.5503 & -1.8651 & 3.7516 & 2.6871 & -1.3903 \\
\hline 0.4581 & 11.6971 & 7.4935 & -6.0781 & 7.0522 & 4.8327 & -3.7461 & 4.9511 & 3.4662 & -2.6197 & 3.7073 & 2.6237 & -1.9554 \\
\hline 0.4686 & 11.5283 & 7.2356 & -8.2043 & 6.9431 & 4.6631 & -5.0527 & 4.8734 & 3.3455 & -3.5370 & 3.6487 & 2.5330 & -2.6424 \\
\hline 0.4779 & 11.2998 & 6.8765 & -10.8985 & 6.7962 & 4.4310 & -6.6996 & 4.7688 & 3.1815 & -4.6925 & 3.5705 & 2.4100 & -3.5078 \\
\hline 0.4861 & 10.9776 & 6.3628 & -14.5700 & 6.5917 & 4.1054 & -8.9309 & 4.6246 & 2.9524 & -6.2574 & 3.4614 & 2.2385 & -4.6796 \\
\hline 0.4935 & 10.4786 & 5.2029 & -20.1275 & 6.2781 & 3.3949 & -12.2895 & 4.4026 & 2.4550 & -8.6118 & 3.2949 & 1.8664 & -6.4424 \\
\hline 0.5000 & 8.6242 & 4.8817 & -122.4056 & 5.1425 & 3.2241 & -73.3139 & 3.6036 & 2.3417 & -51.3745 & 2.6967 & 1.7832 & -38.4432 \\
\hline
\end{tabular}

66 
Table 3.29 Normalized $K_{1}, T_{11}$ and $T_{33}$ for $\mathrm{a} / \mathrm{W}=0.8, \mathrm{t} / \mathrm{W}=4.0, \mathrm{SECP}$

\begin{tabular}{|c|c|c|c|c|c|c|c|c|c|c|c|c|}
\hline \multirow{2}{*}{$z / t$} & \multicolumn{3}{|c|}{ Uniform } & \multicolumn{3}{|c|}{ Linear } & \multicolumn{3}{|c|}{ Parabolic } & \multicolumn{3}{|c|}{ Cubic } \\
\hline & $K_{I}$ & $T_{1 I}$ & $T_{33}$ & $K_{I}$ & $T_{11}$ & $T_{33}$ & $K_{I}$ & $T_{11}$ & $T_{33}$ & $\boldsymbol{K}_{I}$ & $T_{11}$ & $T_{33}$ \\
\hline 0.0000 & 12.0347 & 7.0297 & 2.0917 & 7.2795 & 4.5768 & 1.3603 & 5.1158 & 3.2952 & 0.9788 & 3.8340 & 2.4988 & 0.7420 \\
\hline 0.0615 & 12.0347 & 7.0291 & 2.0901 & 7.2795 & 4.5765 & 1.3593 & 5.1172 & 3.2949 & 0.9780 & 3.8340 & 2.4986 & 0.7414 \\
\hline 0.1161 & 12.0347 & 7.0283 & 2.0858 & 7.2805 & 4.5759 & 1.3562 & 5.1172 & 3.2947 & 0.9758 & 3.8340 & .4984 & 0.7397 \\
\hline 0.1647 & 12.0347 & 7.0273 & 2.0785 & 7.2805 & 4.5754 & 1.3511 & 5.1172 & 3.2943 & 0.9720 & 3.8340 & 2.4981 & 0.7367 \\
\hline 0.2078 & 12.0347 & 7.0269 & 2.0681 & 7.2815 & 4.5753 & 1.3440 & 5.1186 & 3.2943 & 0.9667 & 3.8359 & 2.4981 & 0.7327 \\
\hline 0.2462 & 12.0347 & 7.0265 & 2.0534 & 7.2824 & 4.5753 & 1.3339 & 5.1186 & 3.2945 & 0.9593 & 3.8359 & 2.4984 & 0.7270 \\
\hline 0.2802 & 12.0407 & 7.0293 & 2.0334 & 7.2844 & 4.5777 & 1.3204 & 5.1200 & 3.2963 & 0.9493 & 3.8359 & 2.4998 & 0.7194 \\
\hline 0.3105 & 12.0407 & 7.0365 & 2.0041 & 7.2854 & 4.5829 & 1.3007 & 5.1214 & 3.3003 & 0.9351 & 3.8378 & 2.5029 & 0.7085 \\
\hline 0.3374 & 12.0467 & 7.0531 & 1.9584 & 7.2874 & 4.5945 & 1.2704 & 5.1214 & 3.3087 & 9131 & 3.8378 & 5094 & 0.6919 \\
\hline 0.3613 & 12.0467 & 7.0861 & 1.8823 & 7.2874 & 4.6163 & 1.2206 & 5.1214 & 3.3245 & 0.8774 & 3.8378 & 2.5213 & 0.6648 \\
\hline 0.3825 & 12.0467 & 7.1437 & 1.7518 & 7.2864 & 4.6534 & 1.1360 & 5.1214 & 3.3509 & 0.8171 & 3.8359 & 2.5412 & 0.6194 \\
\hline 0.4014 & 12.0407 & 7.2341 & 1.5302 & 7.2824 & 4.7103 & 0.9939 & 5.1172 & 3.3909 & 0.7161 & 3.8340 & 2.5712 & 0.5434 \\
\hline 0.4182 & 12.0287 & 7.3585 & 1.1670 & 7.2745 & 4.7873 & 0.7627 & 5.1115 & 3.4446 & 0.5524 & 3.8302 & 2.6111 & 0.4205 \\
\hline 0.4331 & 12.0107 & 7.5047 & 0.6005 & 7.2606 & 4.8759 & 0.4046 & 5.1003 & 3.5059 & 0.2995 & 3.8208 & 2.6566 & 0.2309 \\
\hline 0.4463 & 11.9735 & 7.6411 & -0.2384 & 7.2348 & 4.9561 & -0.1230 & 5.0819 & 3.5605 & -0.0724 & 3.8076 & 2.6968 & -0.0477 \\
\hline 0.4581 & 11.9126 & 7.7174 & -1.4263 & 7.1938 & 4.9961 & -0.8664 & 5.0520 & 3.5865 & -0.5957 & 3.7839 & 2.7154 & -0.4396 \\
\hline 0.4686 & 11.8123 & 7.6785 & -3.0577 & 7.1274 & 4.9619 & -1.8825 & 5.0033 & 3.5597 & -1.3103 & 3.7475 & 2.6944 & -0.9747 \\
\hline 0.4779 & 11.6489 & 7.4593 & -5.2861 & 7.0205 & 4.8133 & -3.2633 & 4.9278 & 3.4527 & -2.2803 & 3.6896 & 2.6135 & -1.7011 \\
\hline 0.4861 & 11.3767 & 6.9844 & -8.4261 & 6.8448 & 4.5049 & -5.1961 & 4.8019 & 3.2337 & -3.6371 & 3.5952 & 2.4491 & -2.7171 \\
\hline 0.4935 & 10.8867 & 5.8832 & -13.1871 & 6.5324 & 3.8155 & -8.1047 & 4.5807 & 2.7491 & -5.6771 & 3.4286 & 2.0864 & -4.2448 \\
\hline 0.5000 & 8.8290 & 5.2827 & -93.1709 & 5.2630 & 3.4522 & -55.8460 & 3.6870 & 2.5005 & -39.1241 & 2.7587 & 1.9016 & -29.2724 \\
\hline
\end{tabular}


Table 3.30 Parameters of the empirical formulae for stress intensity factor $K_{\mathrm{I}}$

\begin{tabular}{|c|c|c|c|c|}
\hline & Uniform $(\mathrm{n}=0)$ & Linear $(\mathrm{n}=1)$ & Parabolic $(\mathrm{n}=2)$ & Cubic $(\mathrm{n}=3)$ \\
\hline$a_{n}$ & -3.16746 & -2.46 & -2.15068 & -2.025551 \\
\hline$b_{n}$ & -0.14676 & -0.09 & -0.06753 & -0.05161 \\
\hline$c_{n}$ & 40.54308 & 27.26 & 22.48572 & 20.274559 \\
\hline$d_{n}$ & 0.013603 & 0.007 & 0.00455 & 0.0033272 \\
\hline$e_{n}$ & -109.323 & -73.4 & -59.8064 & -53.26736 \\
\hline$f_{n}$ & 0.59828 & 0.396 & 0.290944 & 0.2264706 \\
\hline$g_{n}$ & 0.012726 & 0.008 & 0.005238 & 0.0038529 \\
\hline$h_{n}$ & 103.5832 & 68.5 & 54.08243 & 46.548953 \\
\hline$i_{n}$ & -0.92723 & -0.6 & -0.43478 & -0.333507 \\
\hline$j_{n}$ & -0.01347 & -0.01 & -0.00725 & -0.005393 \\
\hline
\end{tabular}




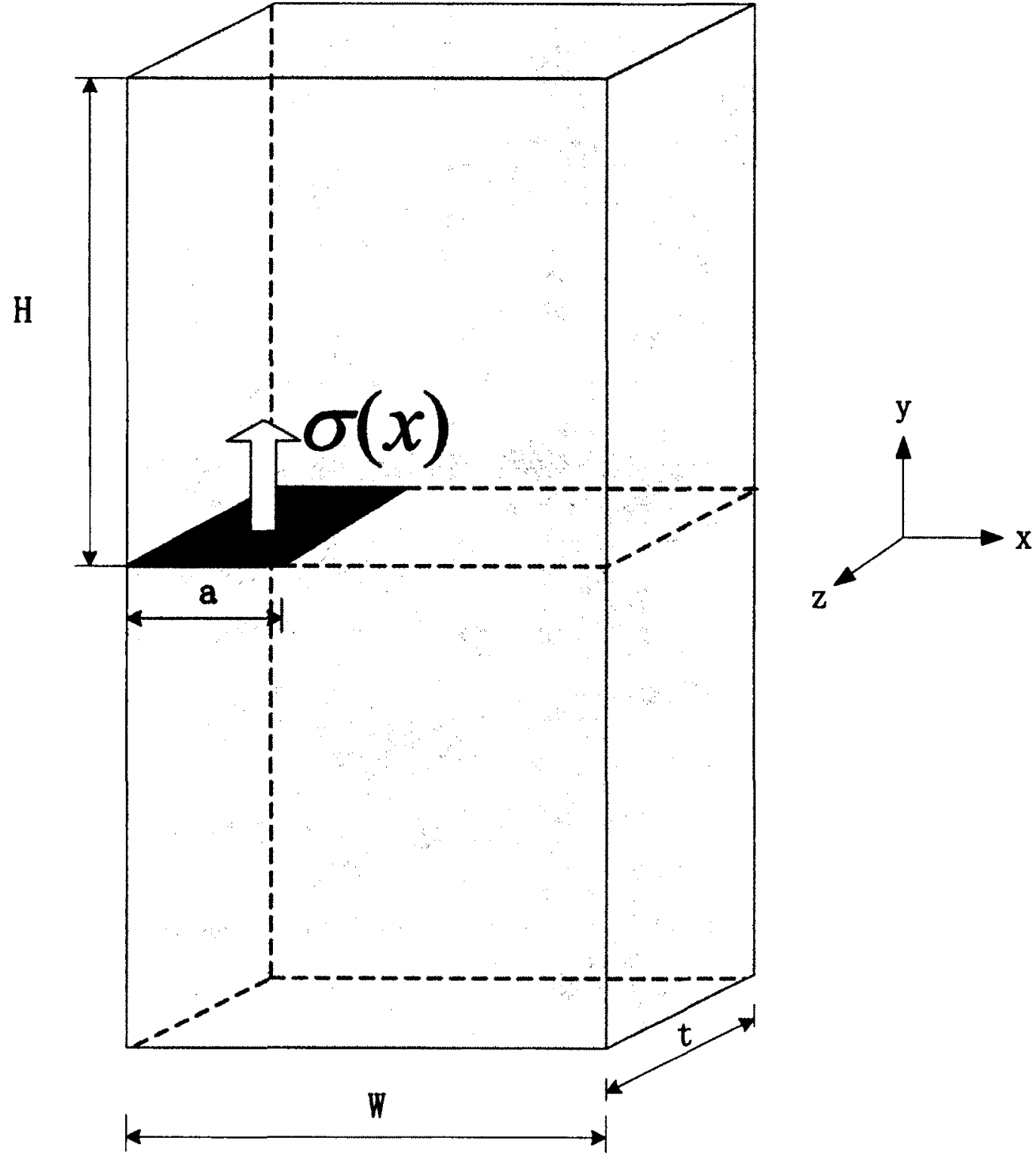

Figure 3.1 Geometric parameters of single edge cracked plate specimen with crack surface subjected to stress distribution $\sigma(x)$ 

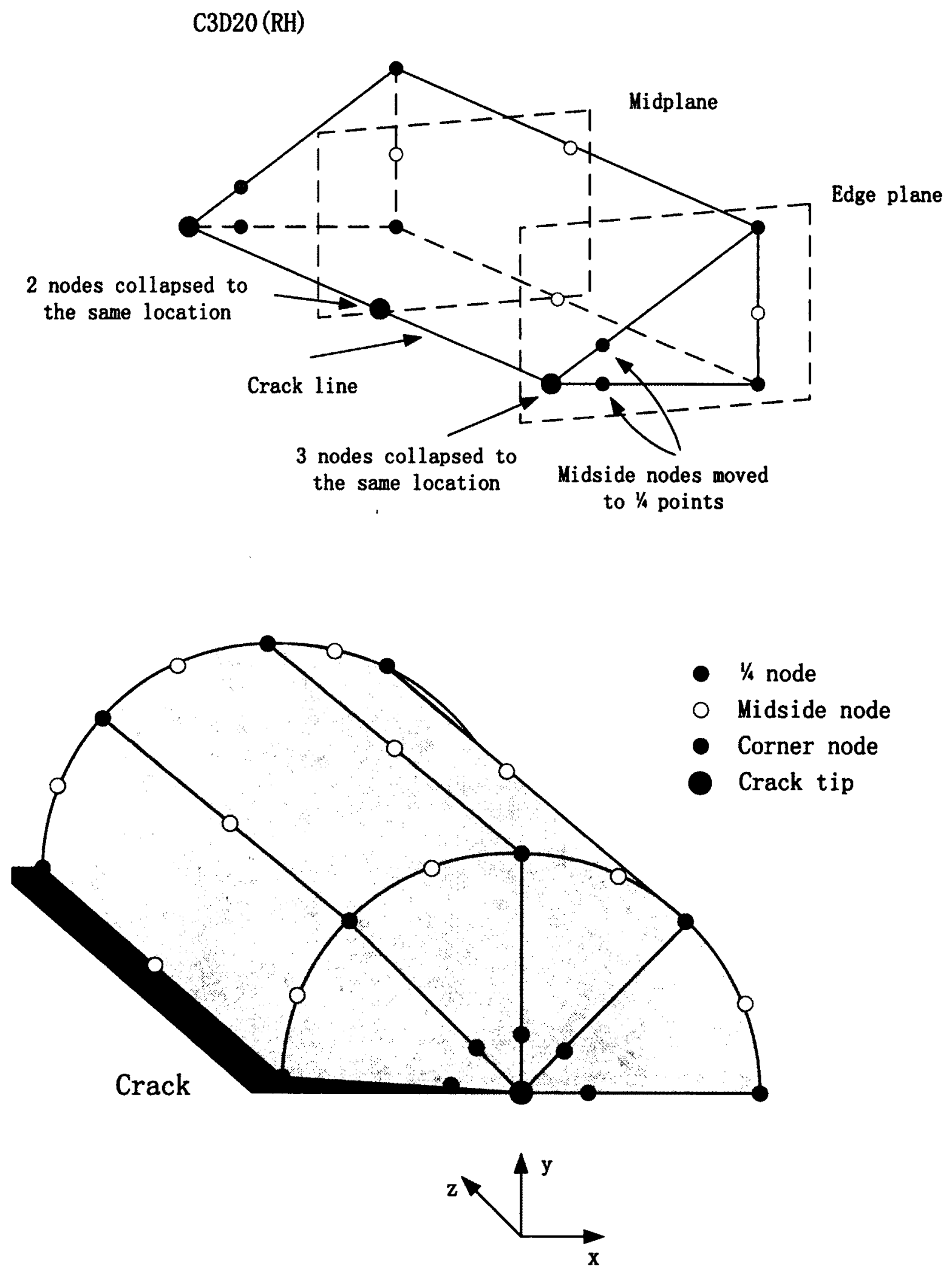

Figure 3.2 C3D20 element and mesh configuration near the crack tip 


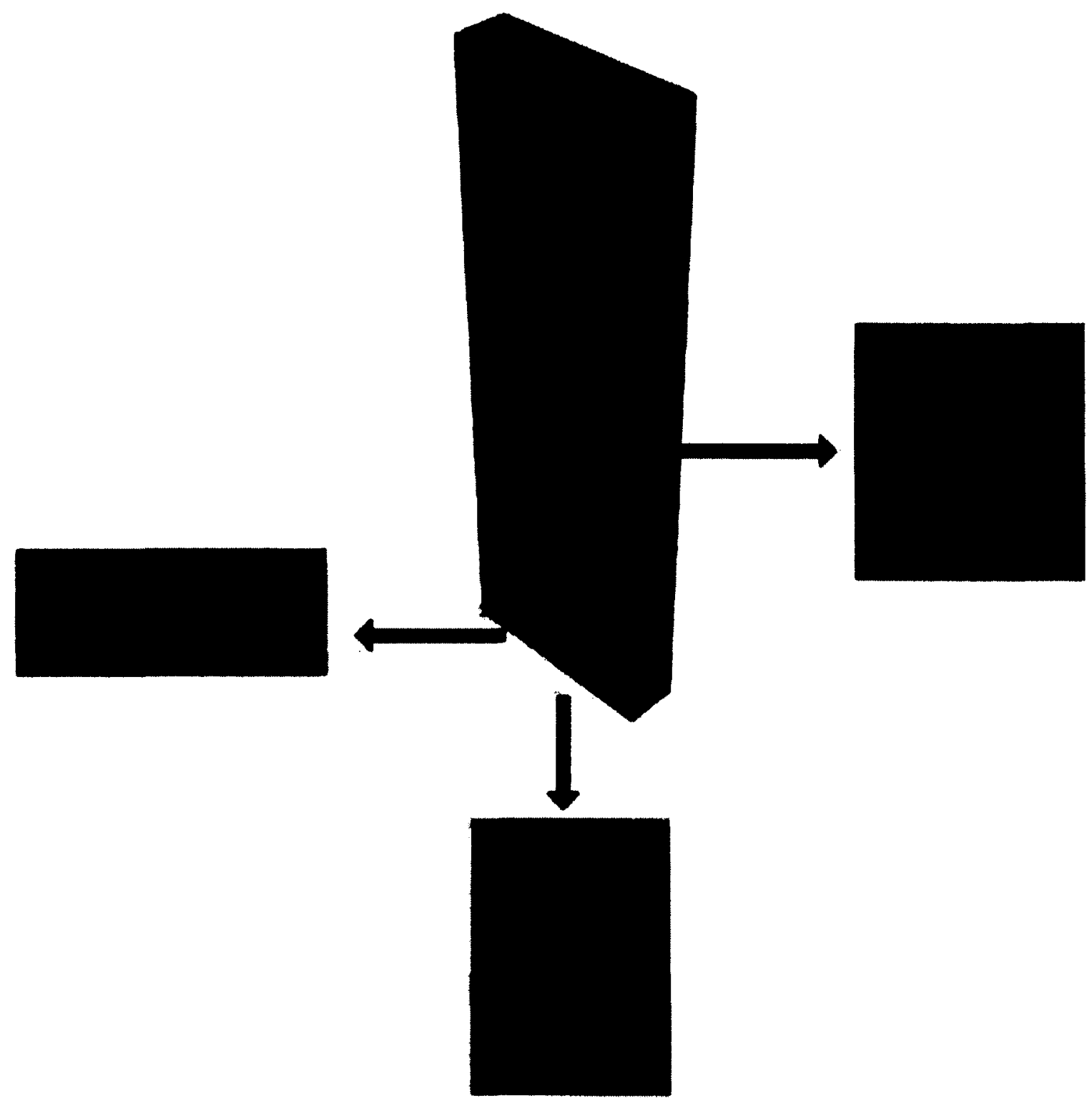

Figure 3.3 Finite element model for $a / W=0.4, t / W=0.5$, SECP 


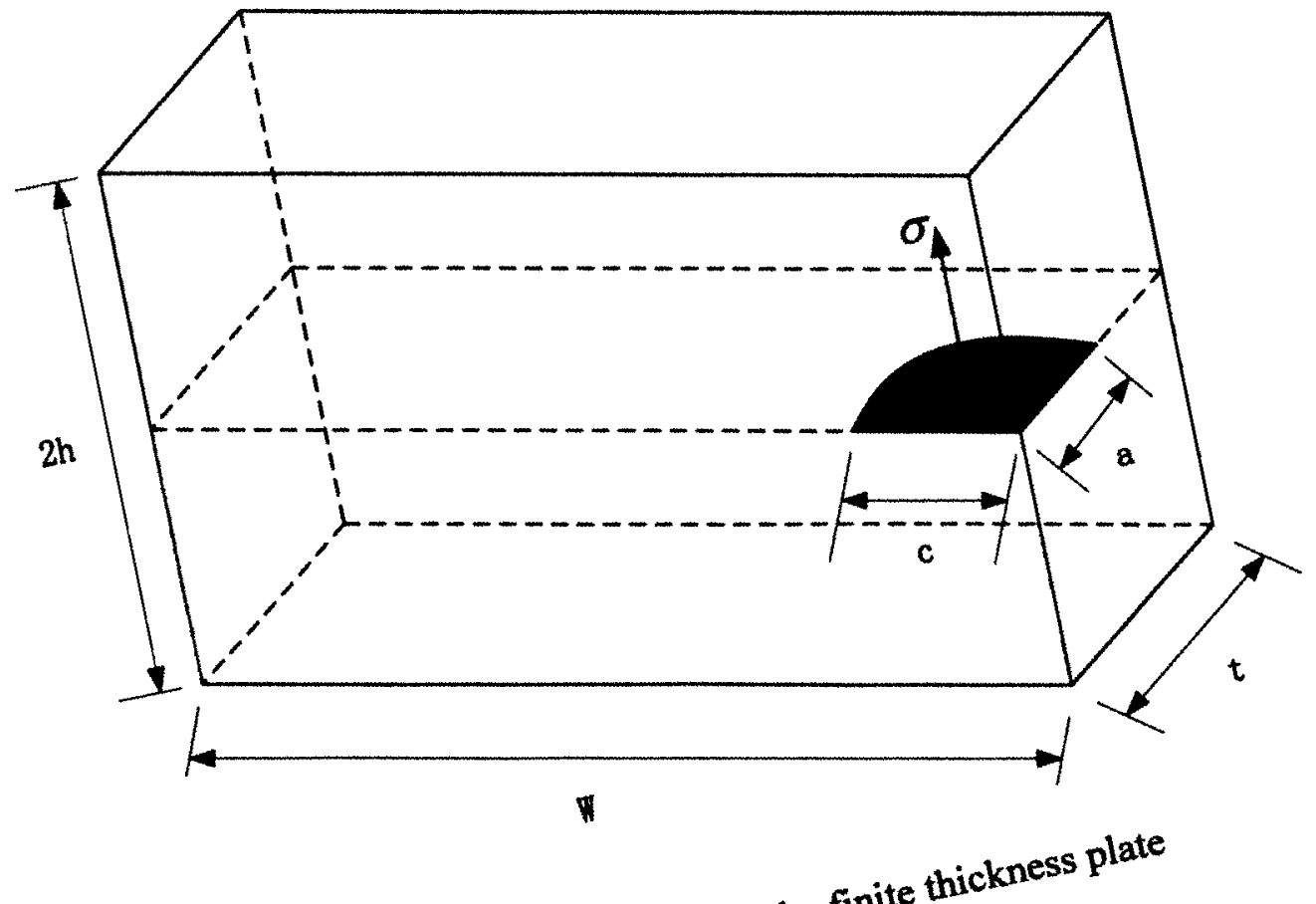

Figure 3.4 Corner crack in the finite thickness plate

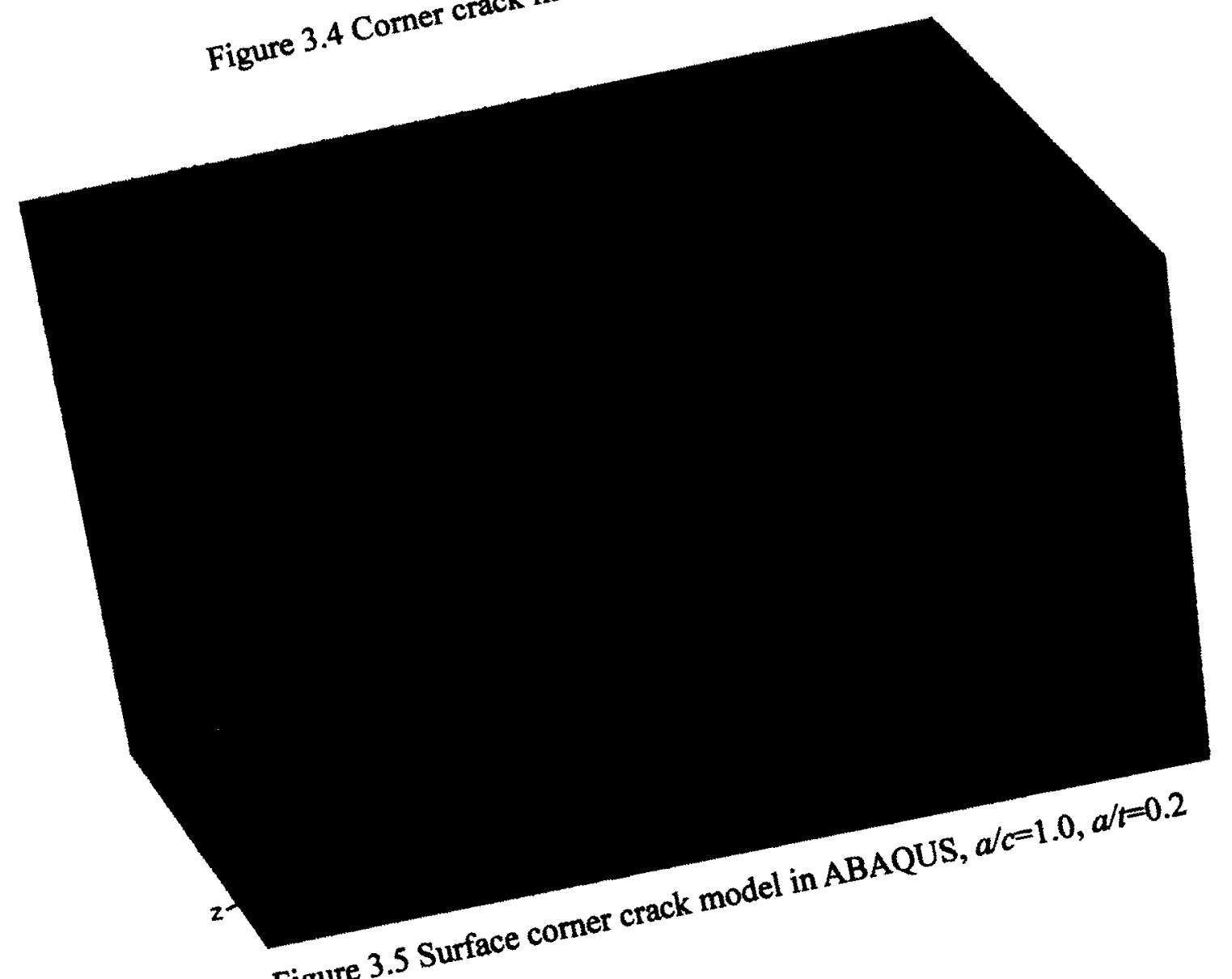




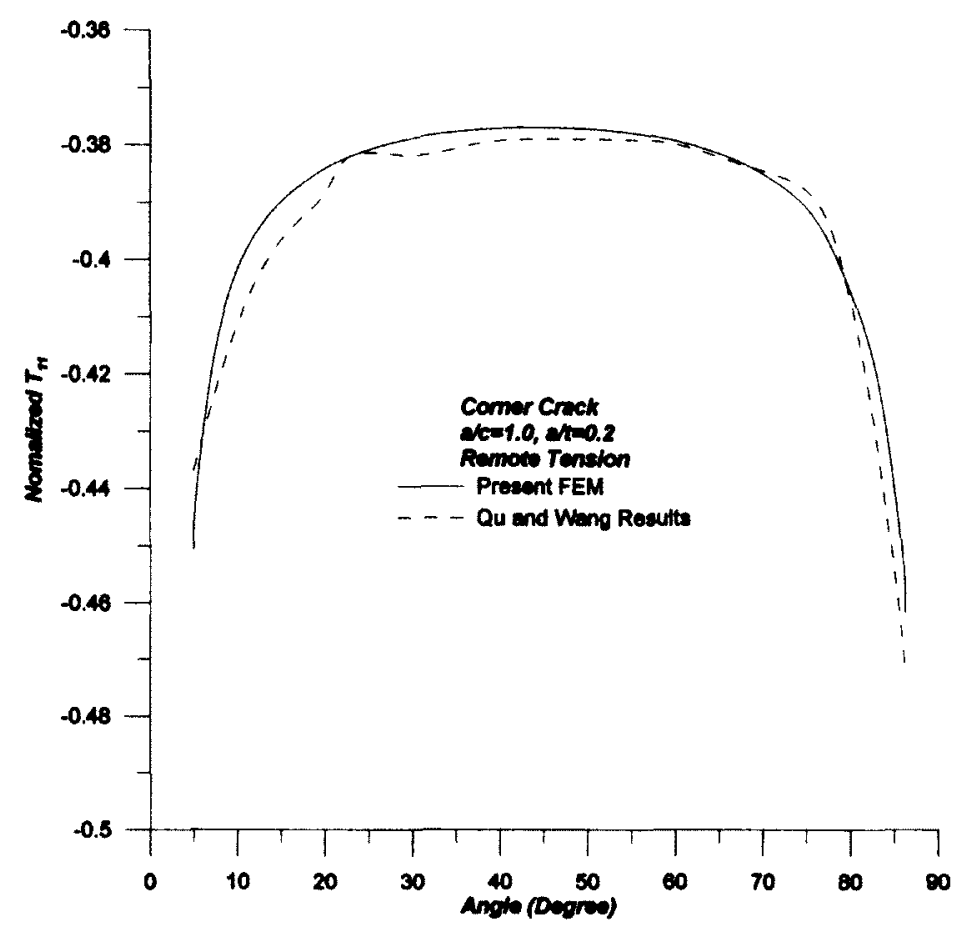

Figure 3.6 Comparison of the $T_{11}$ from present FEM calculation and solution form $\mathrm{Qu}$ and Wang (2006) for surface corner crack, $a / c=1.0, a / t=0.2$

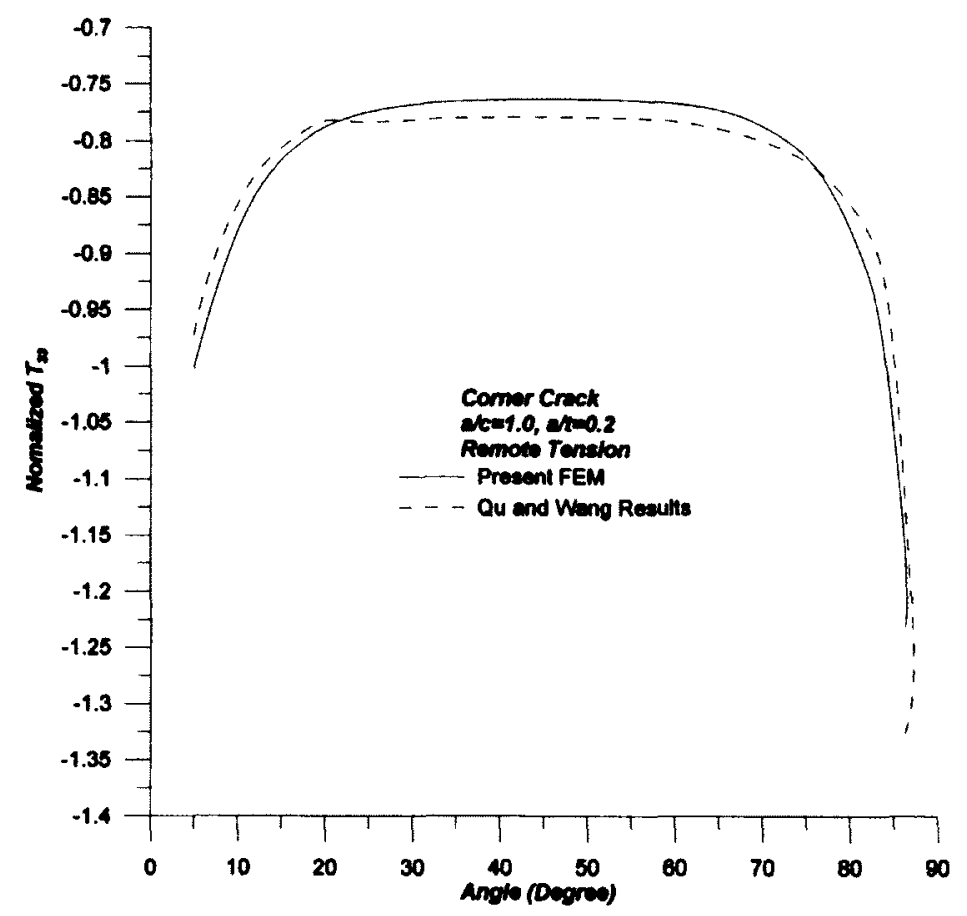

Figure 3.7 Comparison of the $T_{33}$ from present FEM calculation and solution form Qu and Wang (2006) for surface corner crack, $a / c=1.0, a / t=0.2$ 


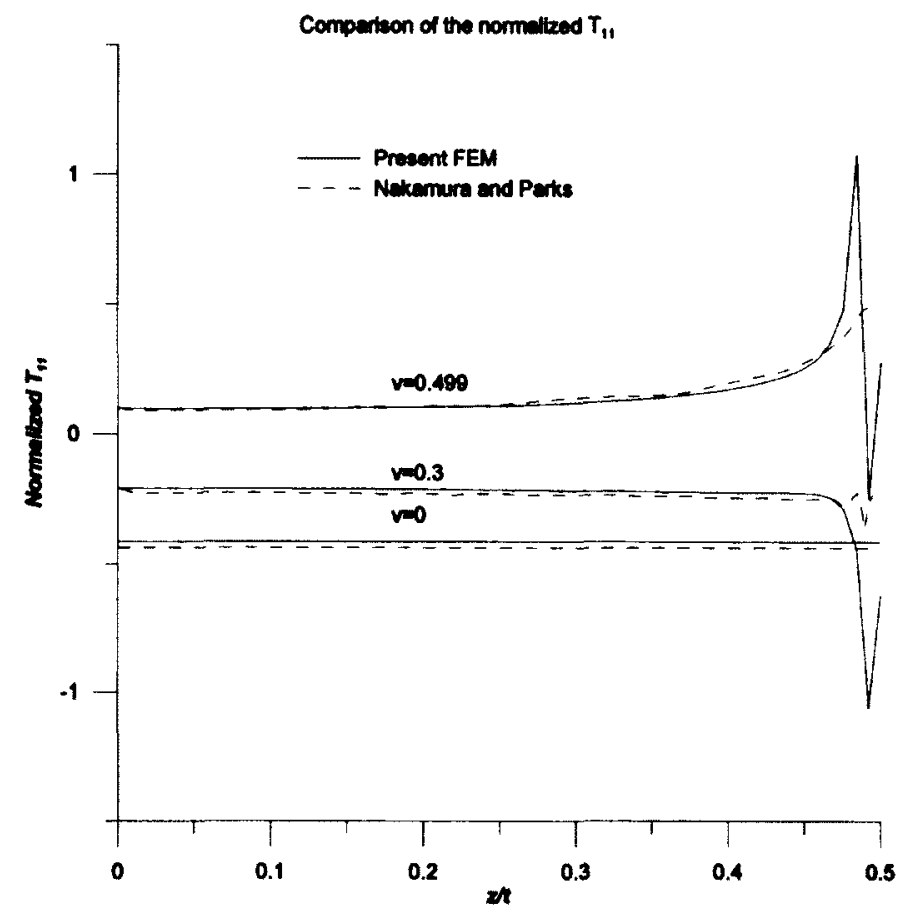

Figure 3.8 Variation of normalized $T_{11}$ along front of an edge-cracked plate for various Poisson's ratios under tension 


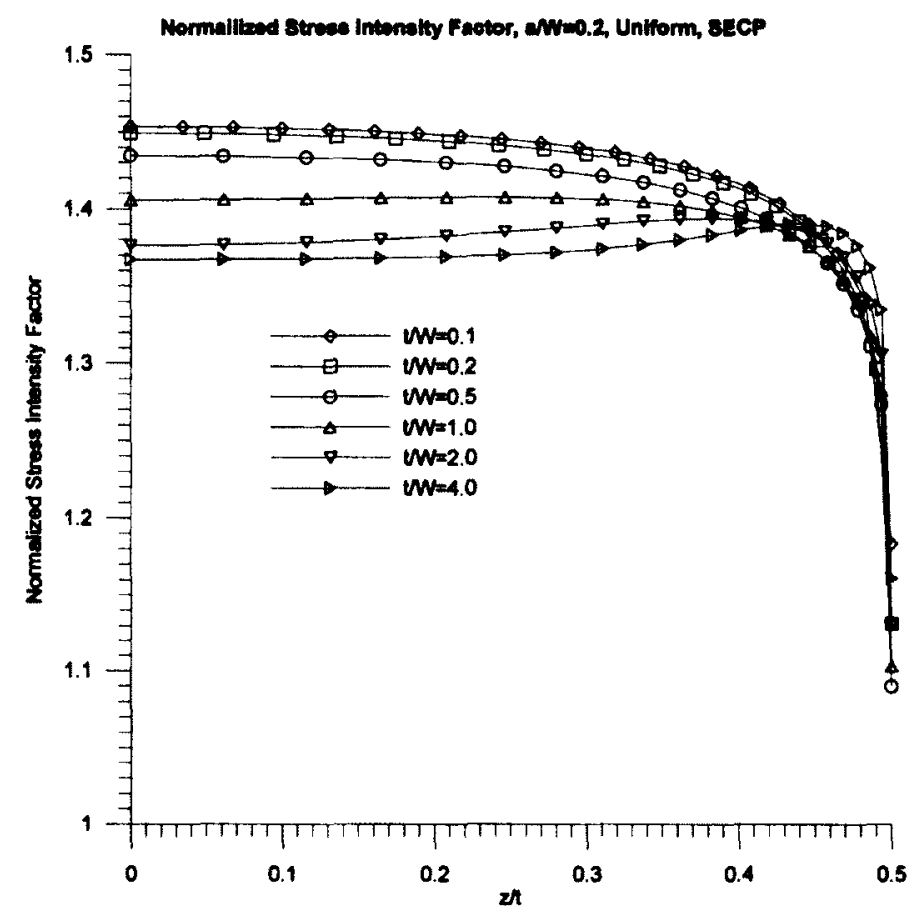

Figure 3.9 Normalized Stress Intensity Factor, $a / W=0.2$, Uniform, SECP

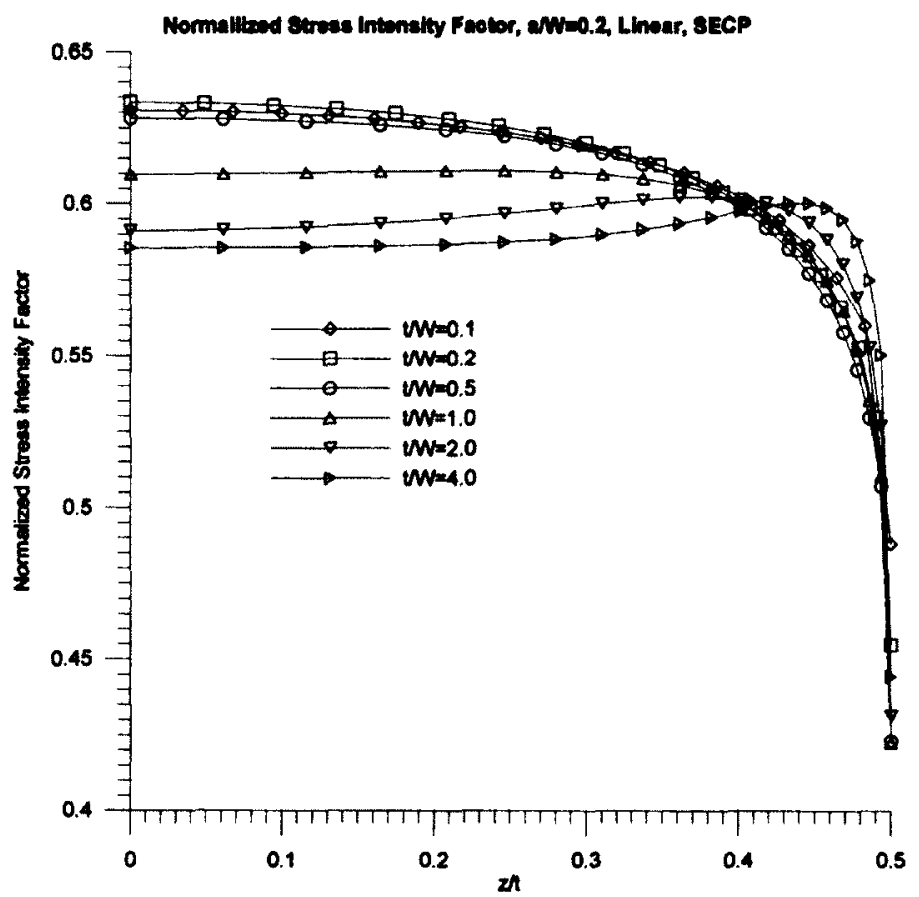

Figure 3.10 Normalized Stress Intensity Factor, $a / W=0.2$, Linear, SECP 


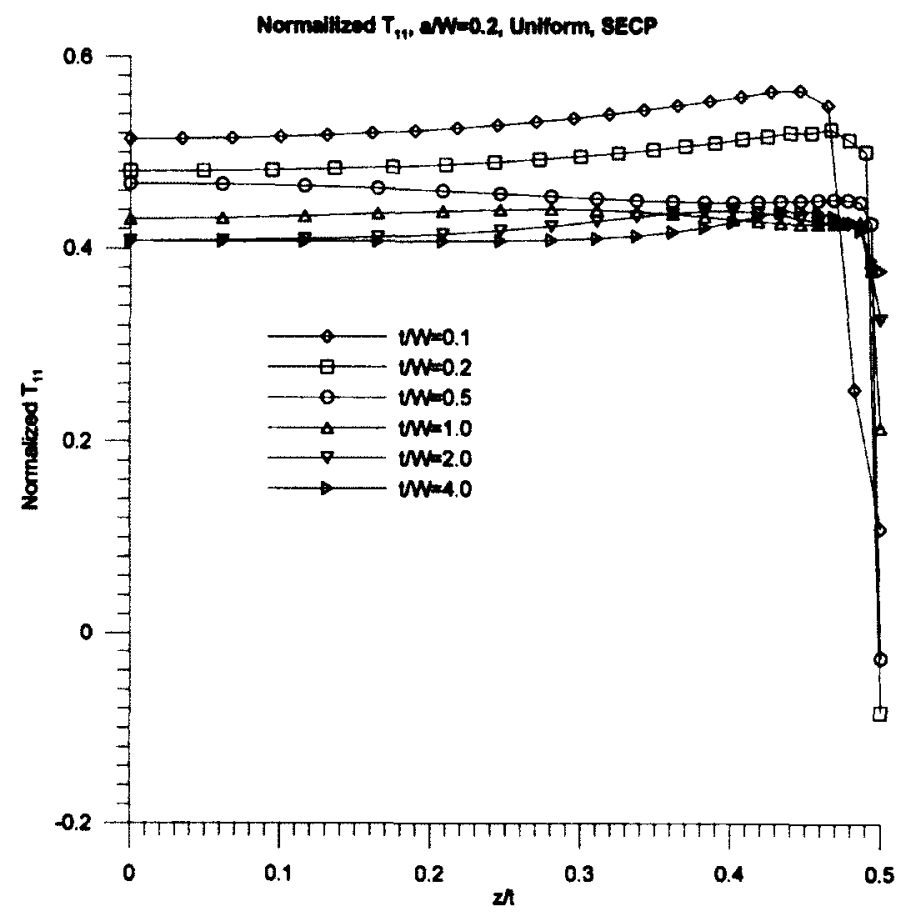

Figure 3.11 Normalized $T_{11}, a / W=0.2$, Uniform, SECP

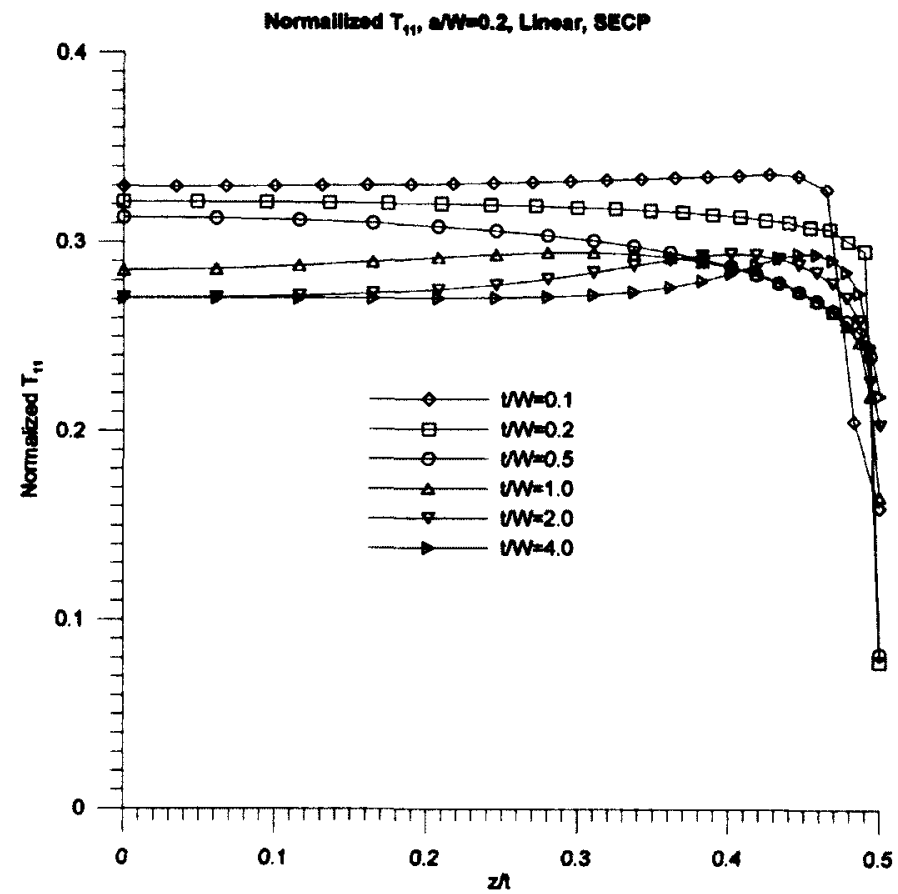

Figure 3.12 Normalized $T_{11}, a / W=0.2$, Linear, SECP 


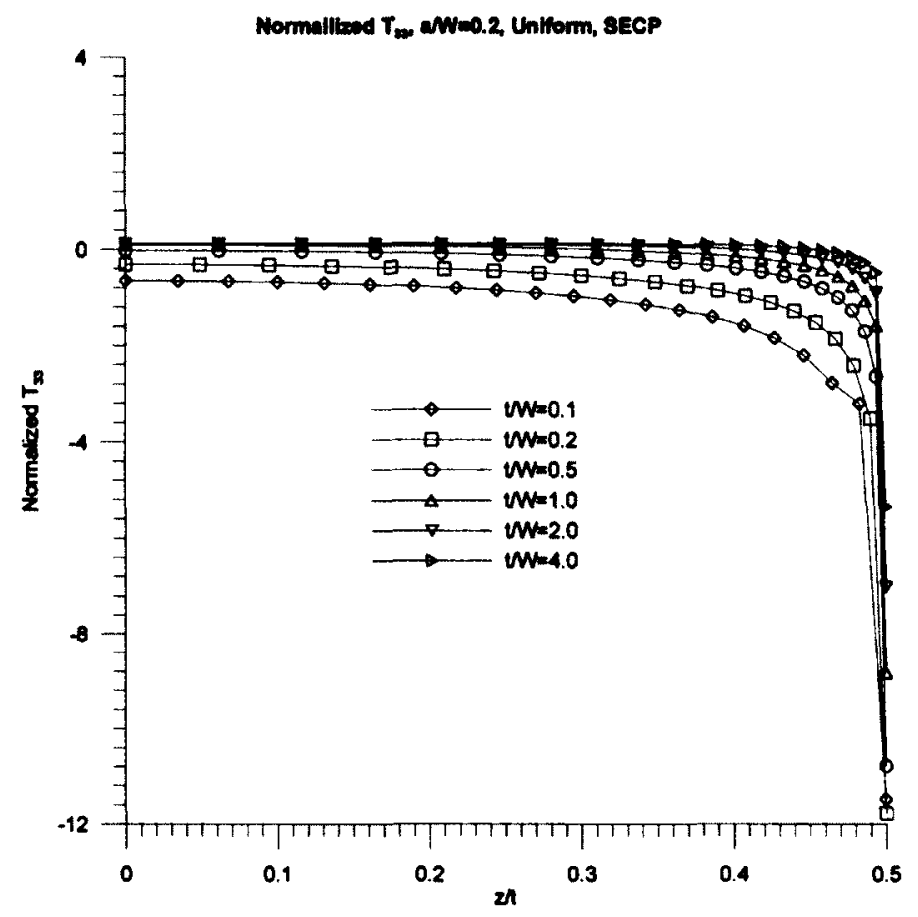

Figure 3.13 Normalized $T_{33}, a / W=0.2$, Uniform, SECP

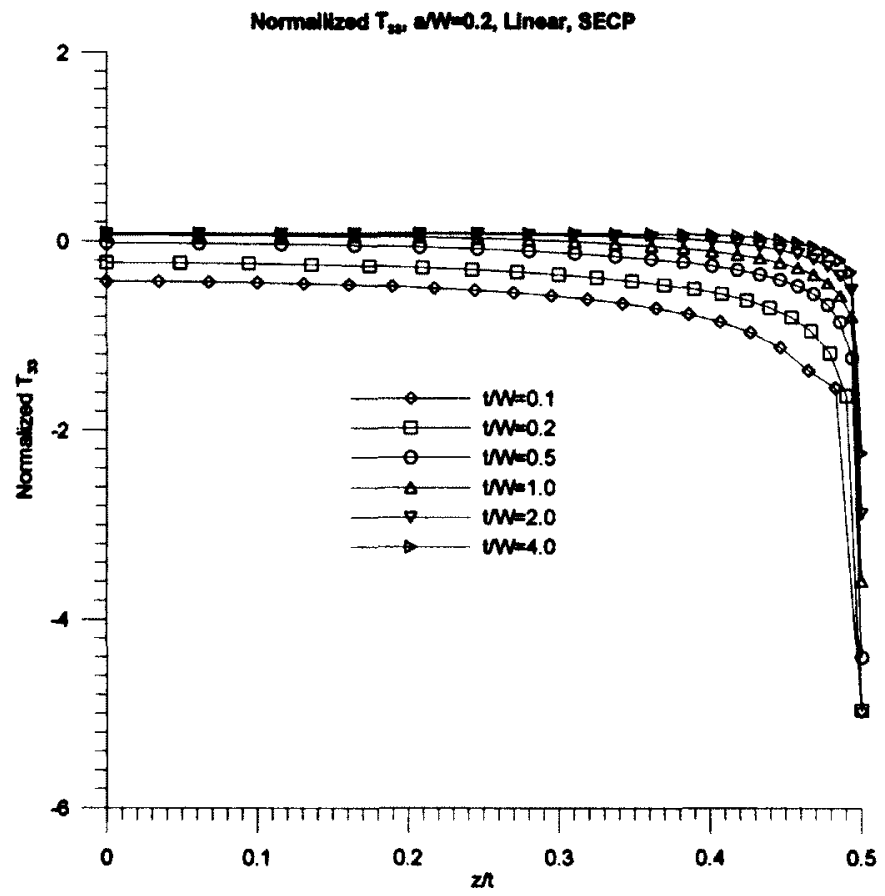

Figure 3.14 Normalized $T_{33}, a / W=0.2$, Linear, SECP 


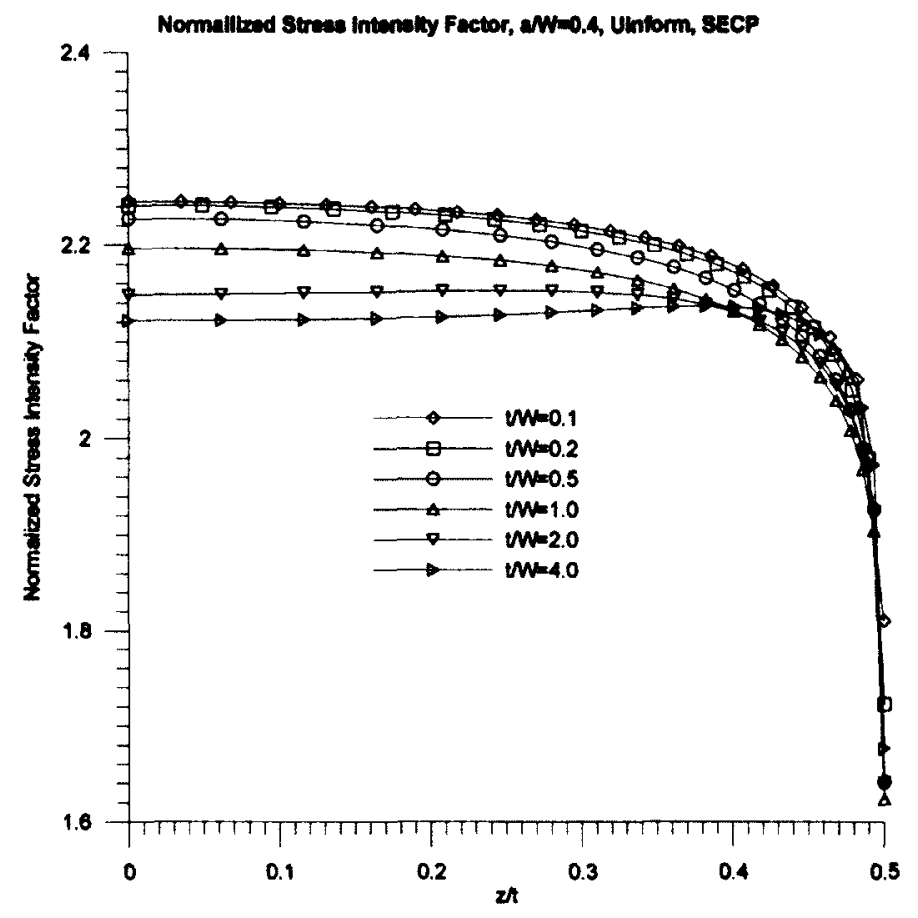

Figure 3.15 Normalized Stress Intensity Factor, $a / W=0.4$, Uniform, SECP

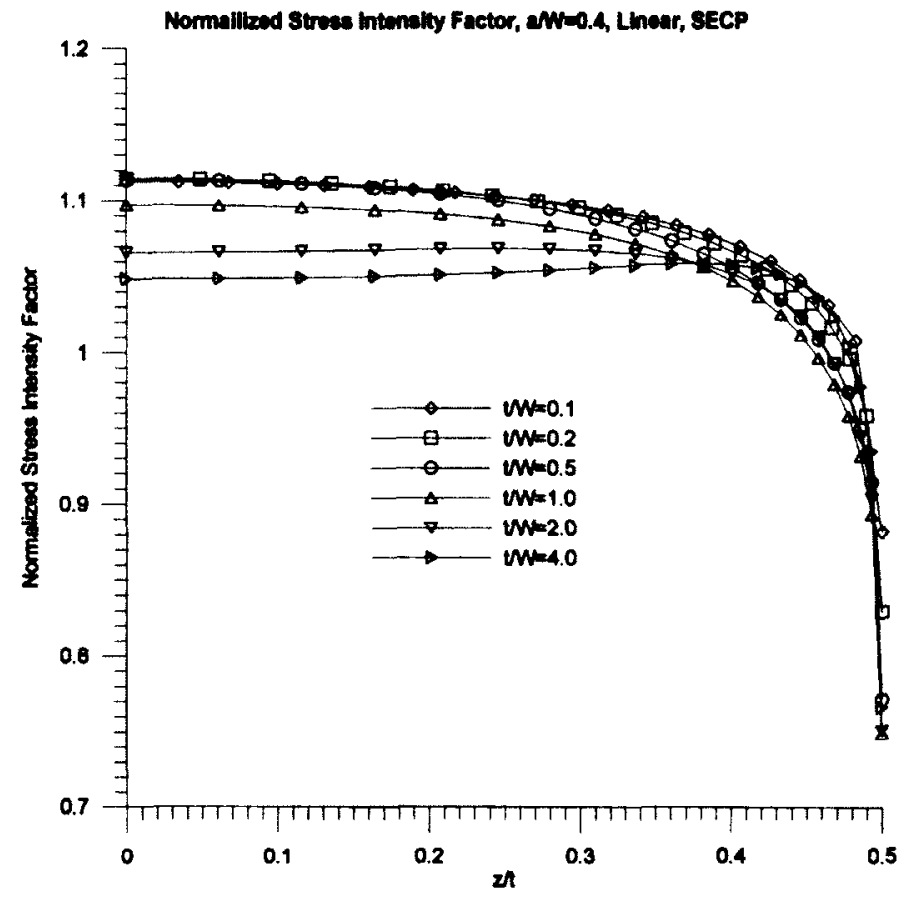

Figure 3.16 Normalized Stress Intensity Factor, $a / W=0.4$, Linear, SECP 


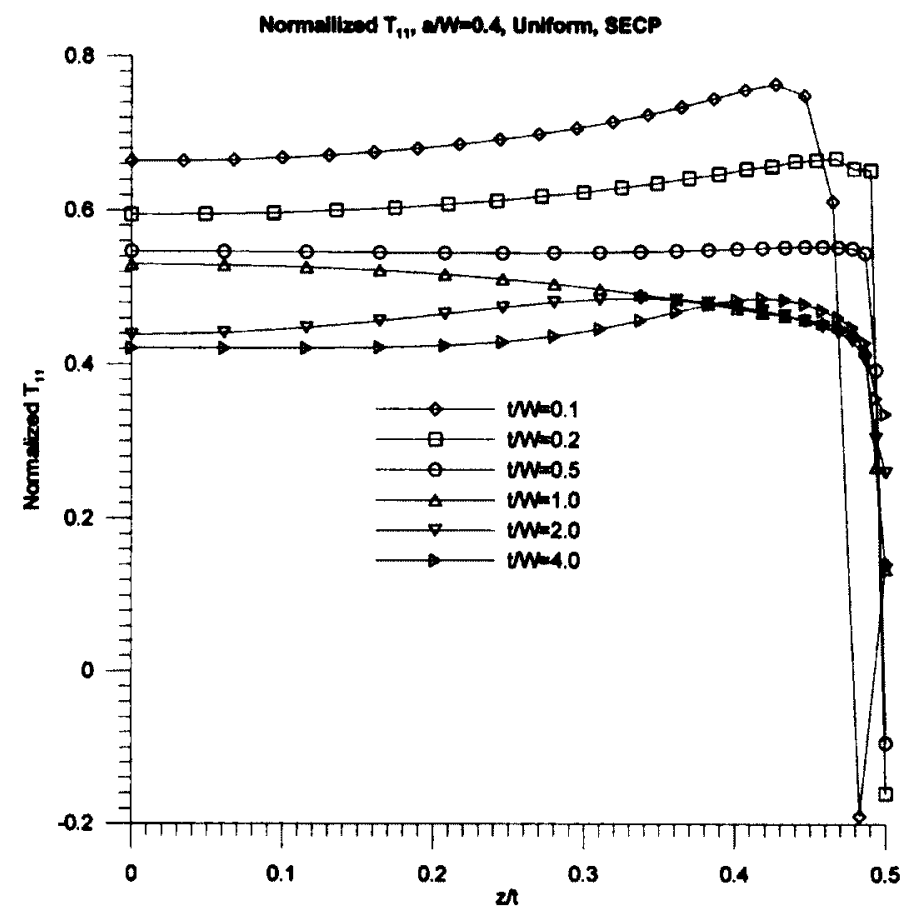

Figure 3.17 Normalized $T_{11}, a / W=0.4$, Uniform, SECP

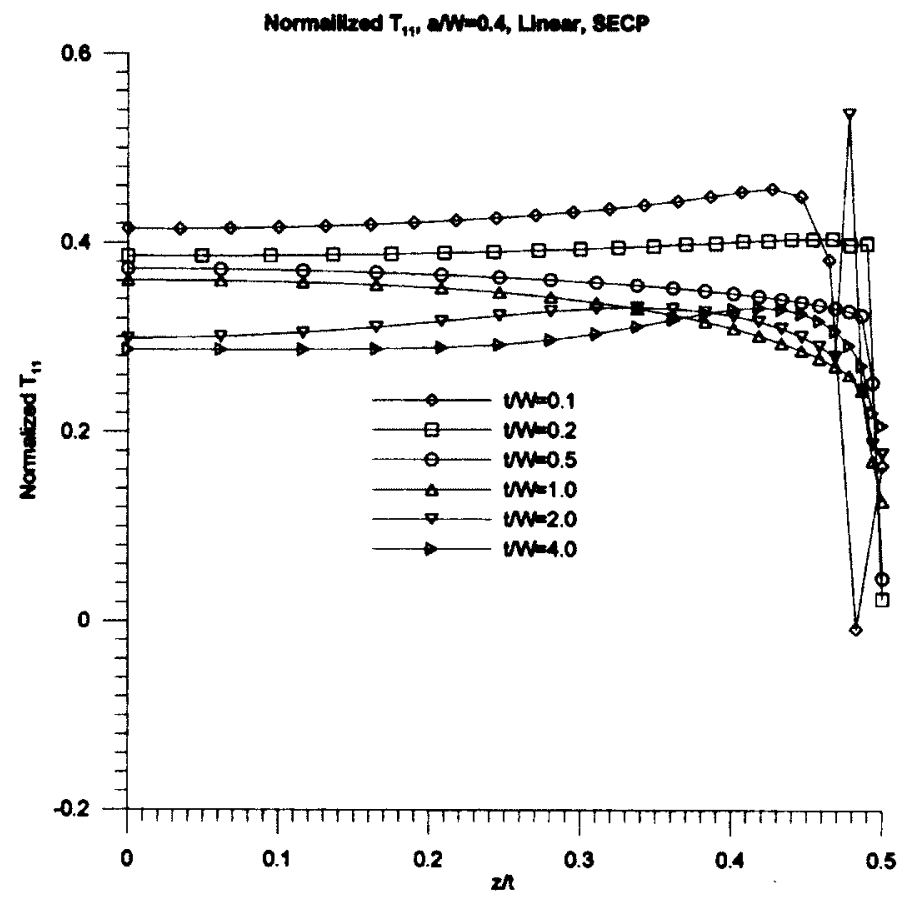

Figure 3.18 Normalized $T_{11}, a / W=0.4$, Linear, SECP 


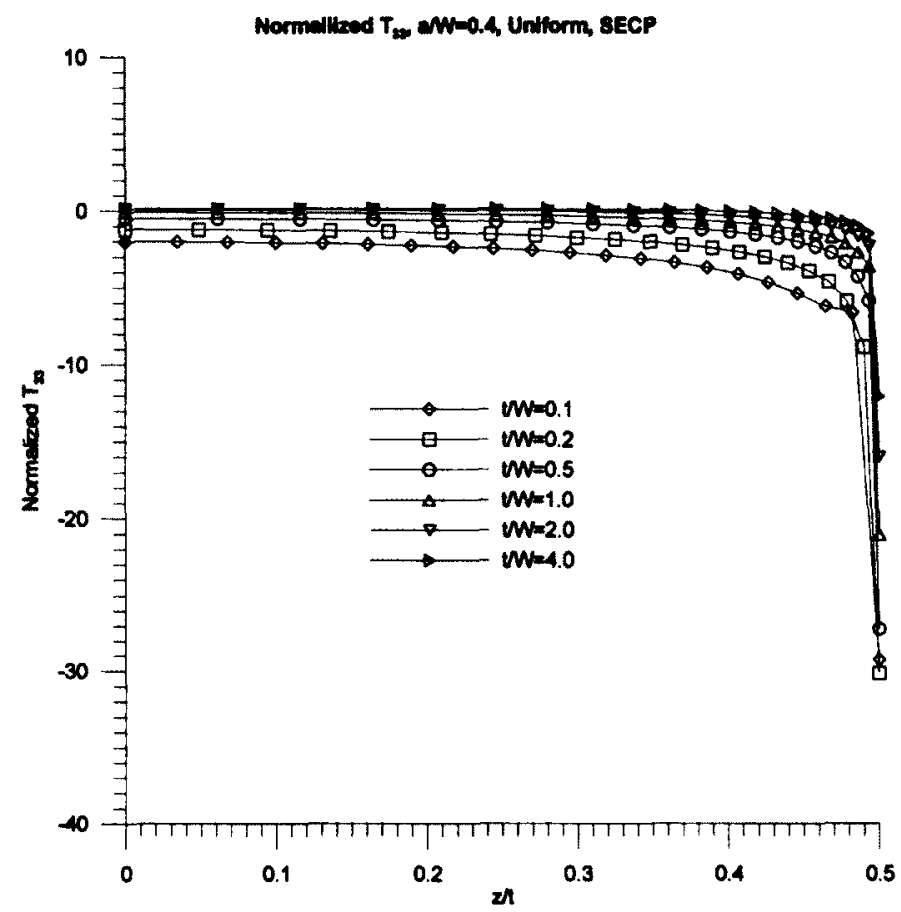

Figure 3.19 Normalized $T_{33}, a / W=0.4$, Uniform, SECP

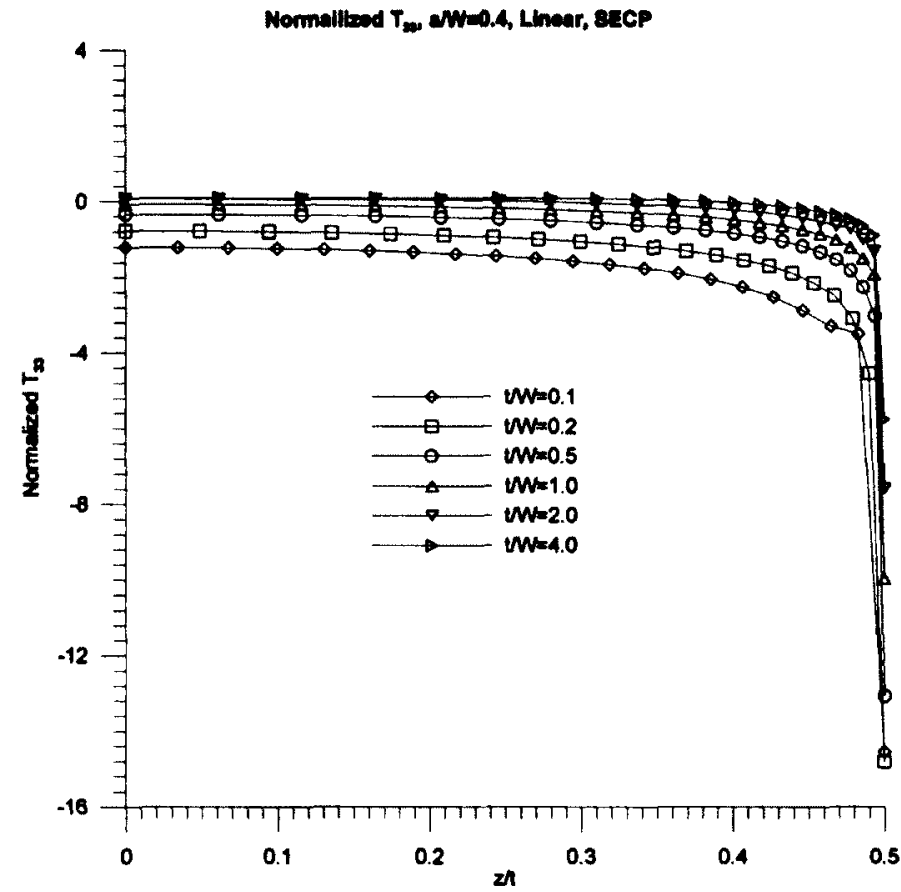

Figure 3.20 Normalized $T_{33}, a / W=0.4$, Linear, SECP 


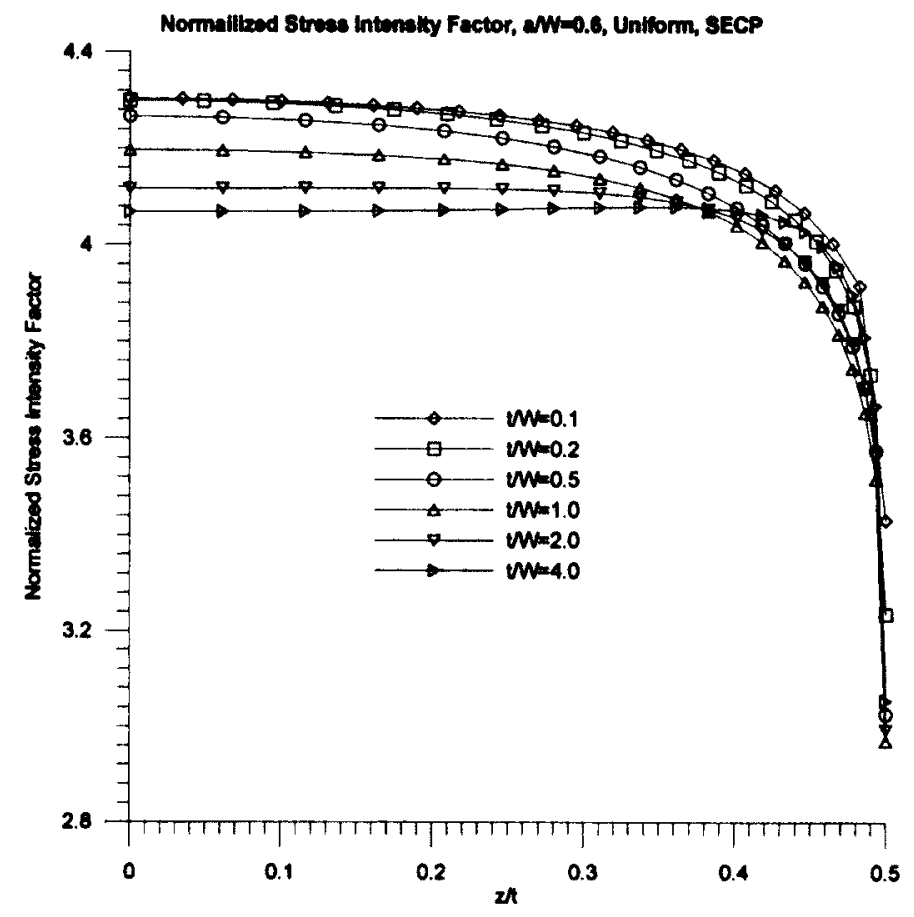

Figure 3.21 Normalized Stress Intensity Factor, $a / W=0.6$, Uniform, SECP

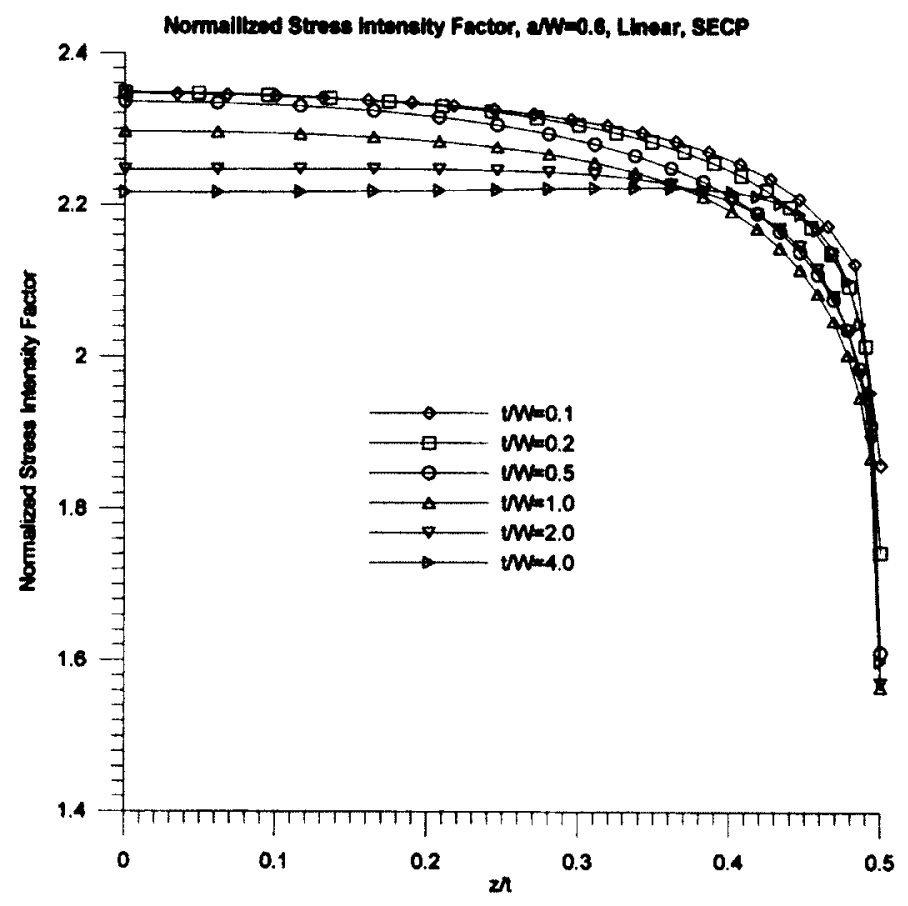

Figure 3.22 Normalized Stress Intensity Factor, $a / W=0.6$, Linear, SECP 


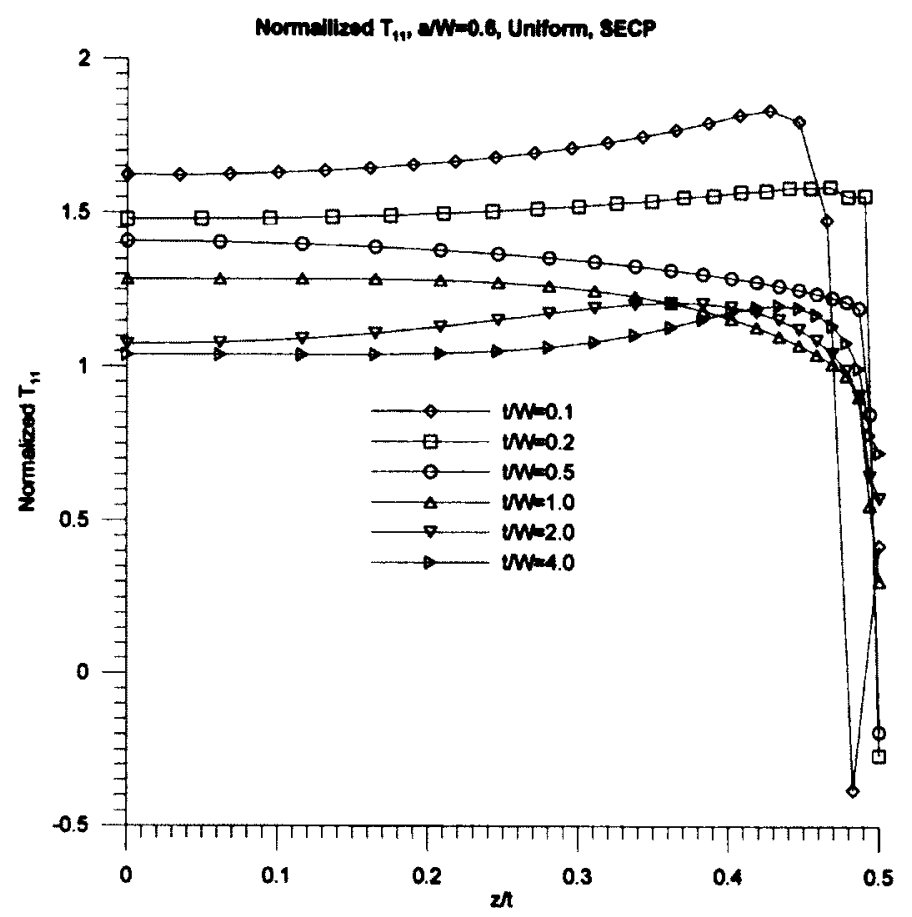

Figure 3.23 Normalized $T_{11}, a / W=0.6$, Uniform, SECP

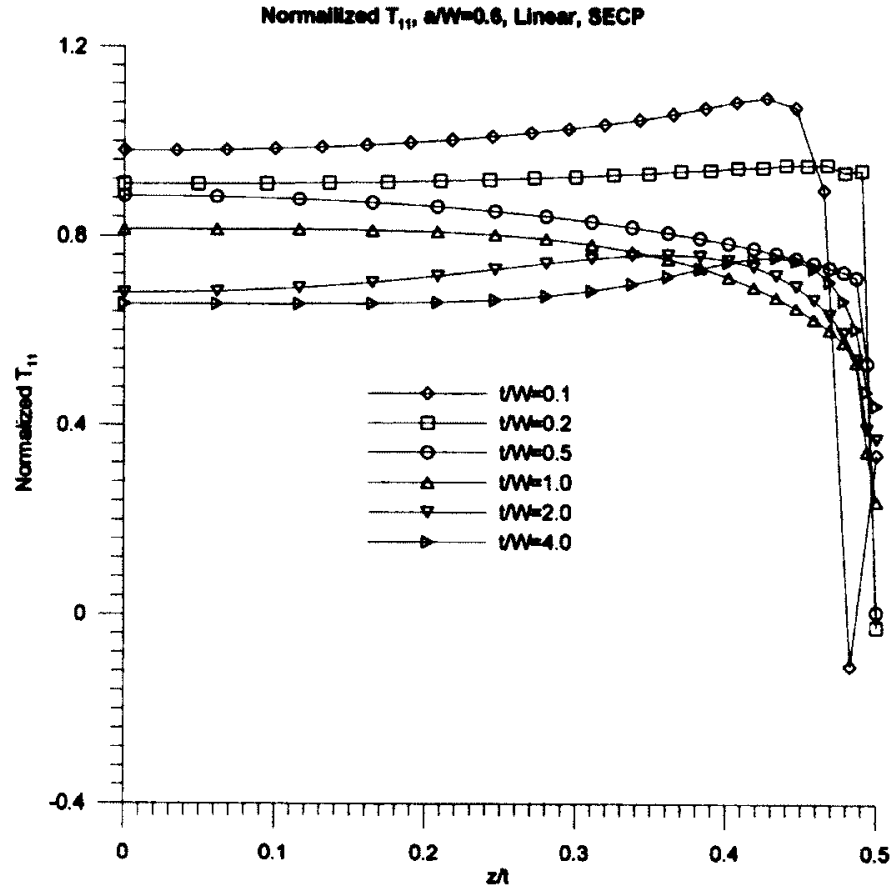

Figure 3. 24 Normalized $T_{11}, a / W=0.6$, Linear, SECP 


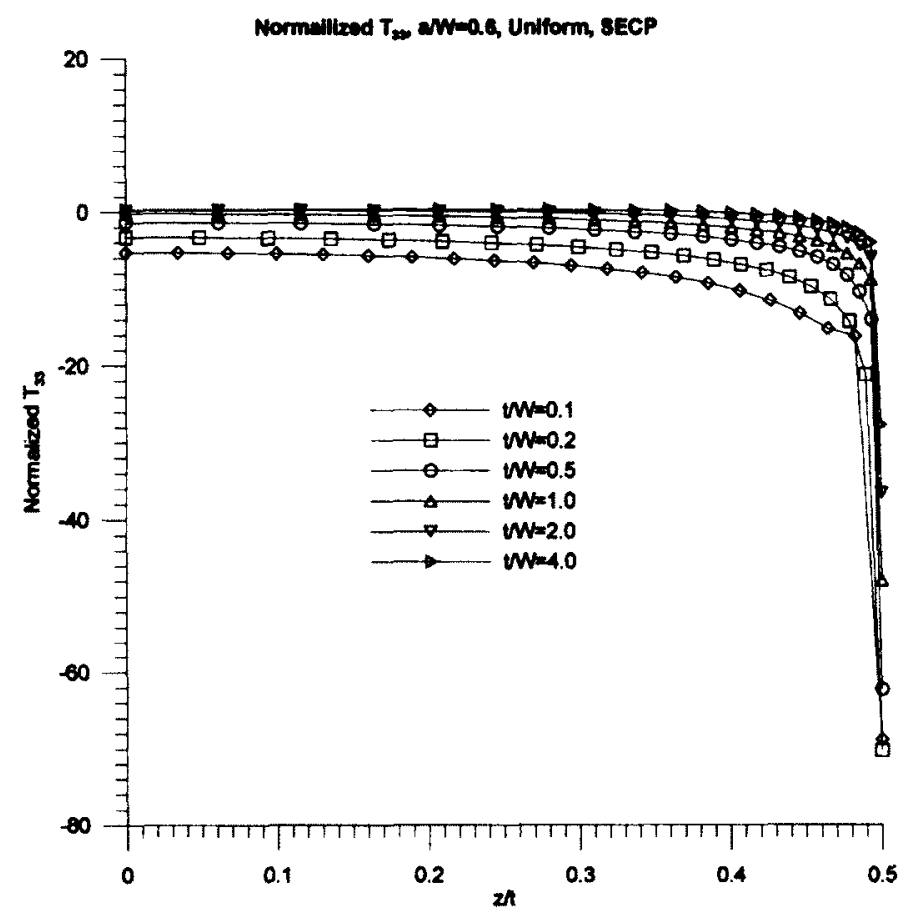

Figure 3.25 Normalized $T_{33}, a / W=0.6$, Uniform, SECP

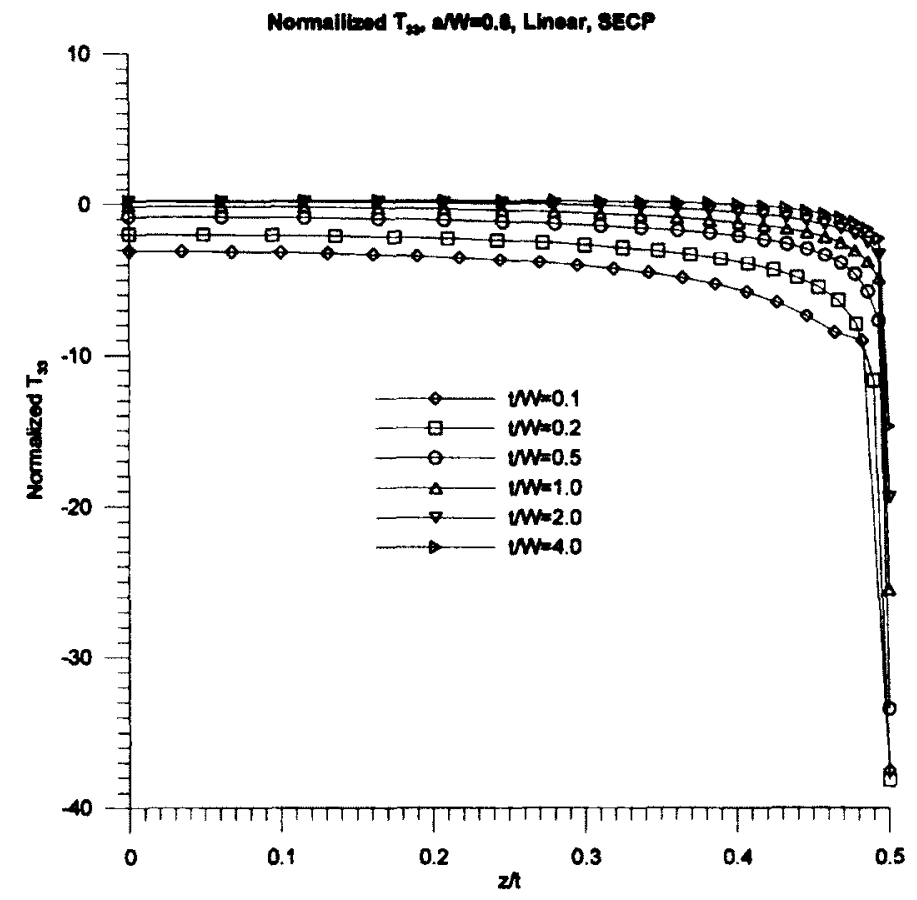

Figure 3.26 Normalized $T_{33}, a / W=0.6$, Linear, SECP 


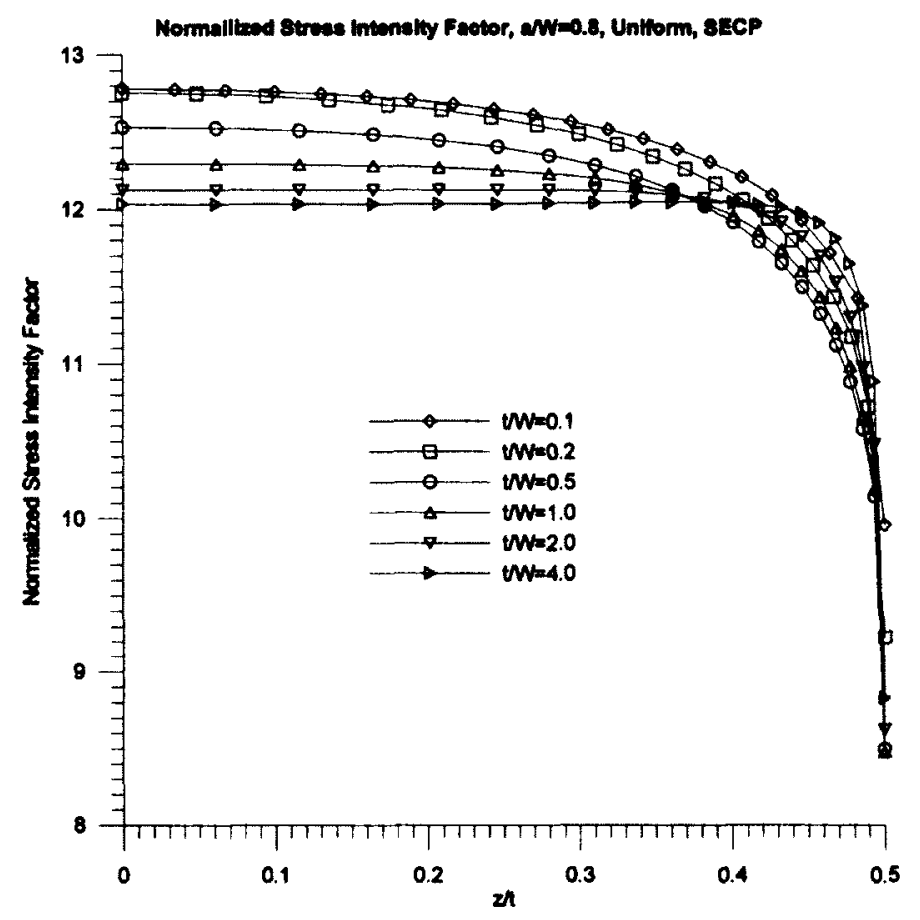

Figure 3.27 Normalized Stress Intensity Factor, $a / W=0.8$, Uniform, SECP

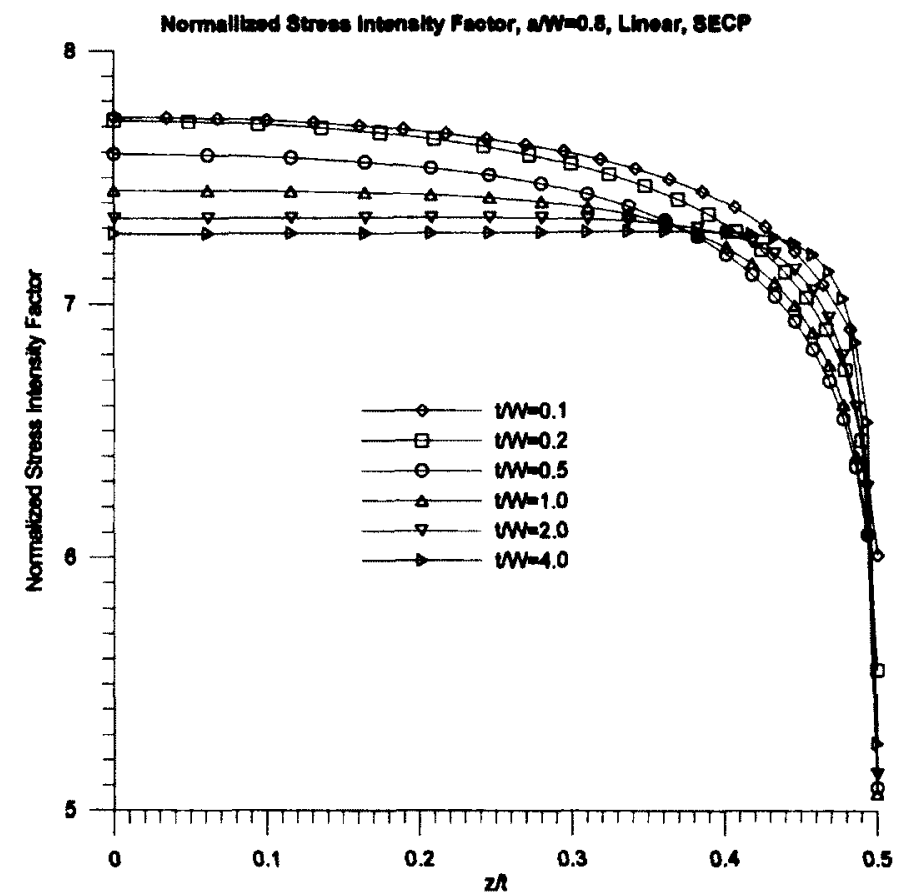

Figure 3.28 Normalized Stress Intensity Factor, $a / W=0.8$, Linear, SECP 


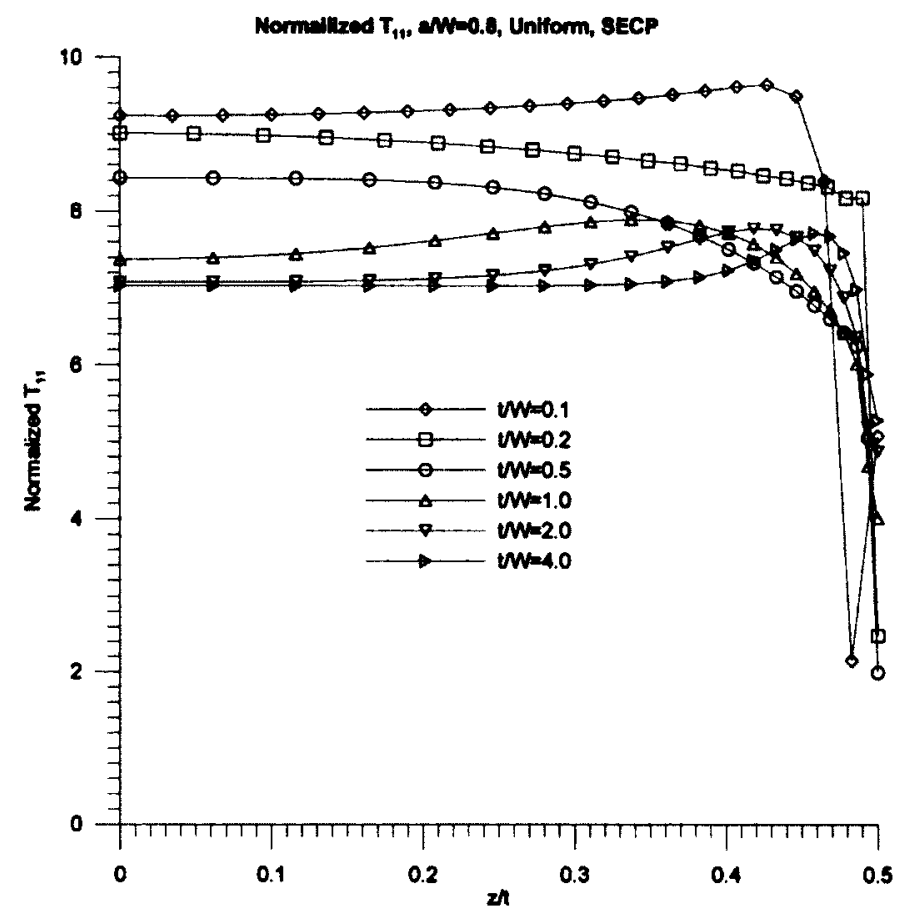

Figure 3.29 Normalized $T_{11}, a / W=0.8$, Uniform, SECP

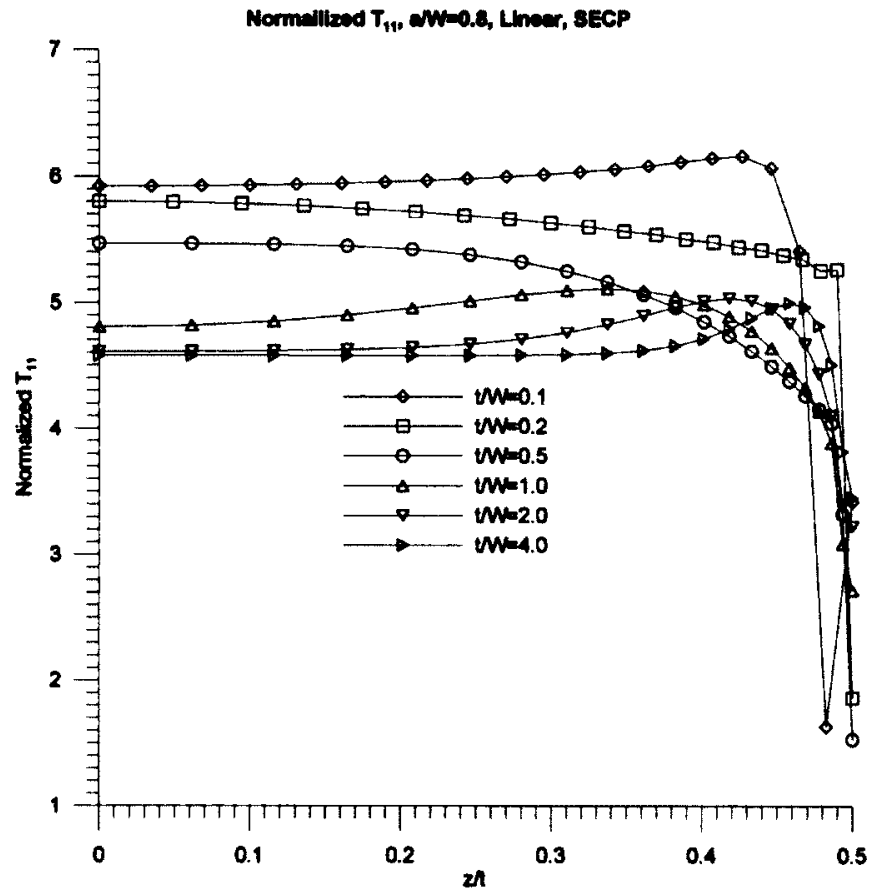

Figure 3.30 Normalized $T_{11}, a / W=0.8$, Linear, SECP 


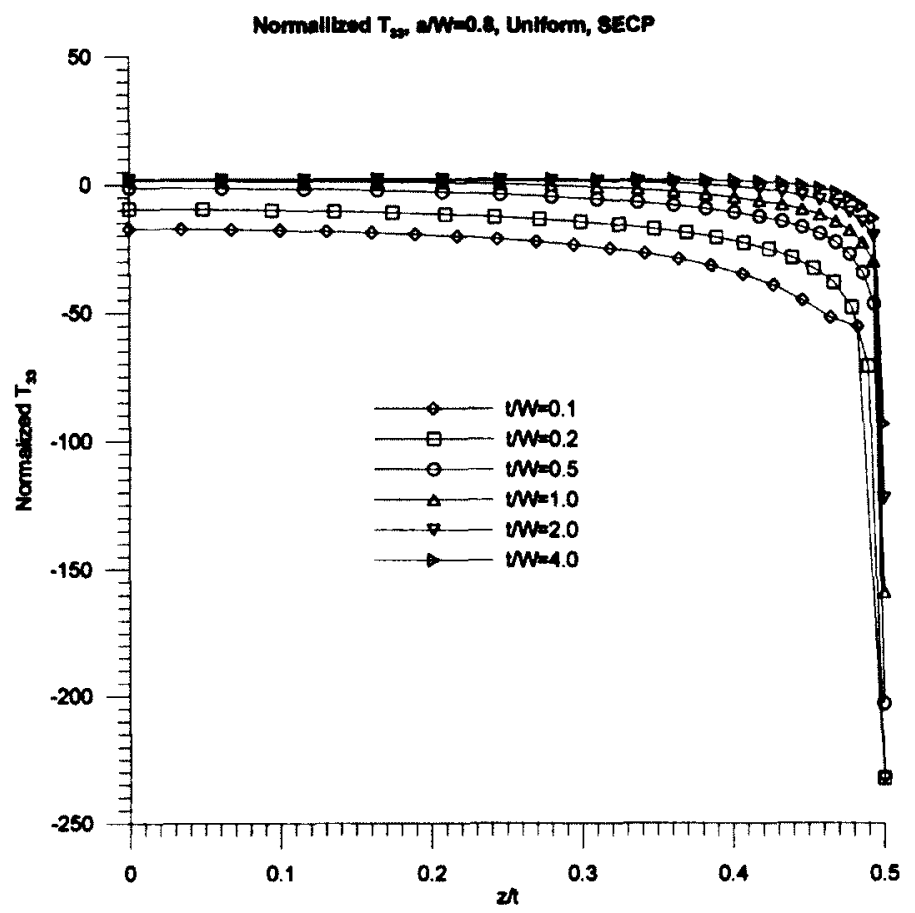

Figure 3.31 Normalized $T_{33}, a / W=0.8$, Uniform, SECP

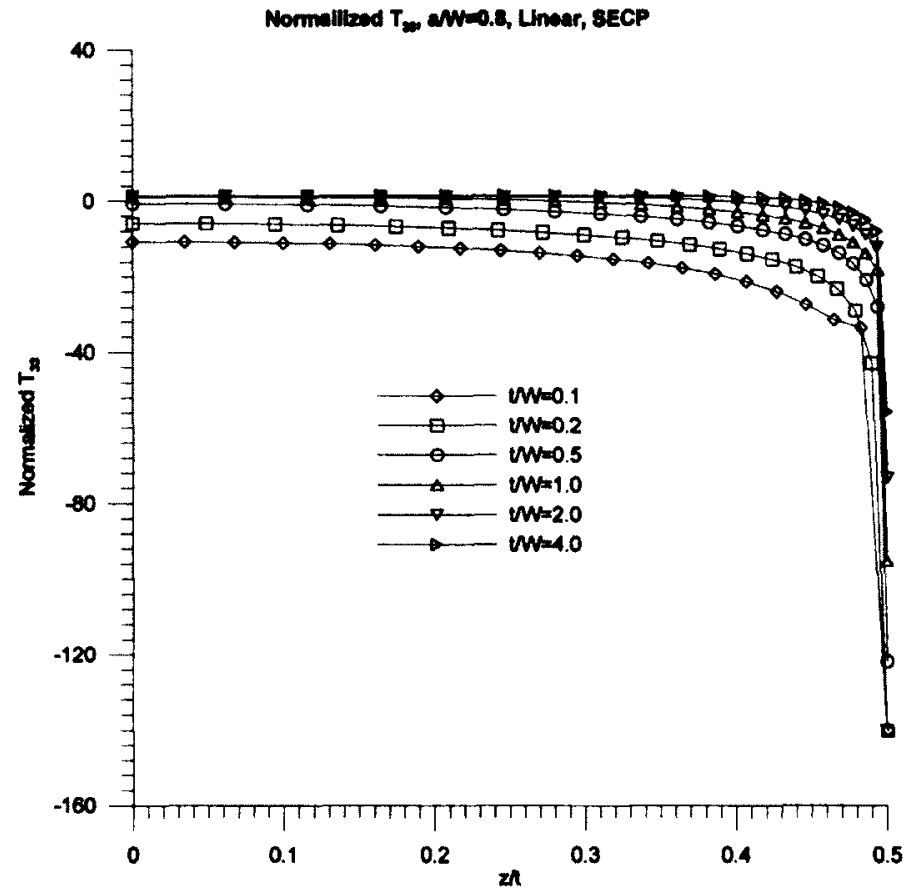

Figure 3.32 Normalized $T_{33}, a / W=0.8$, Linear, SECP 


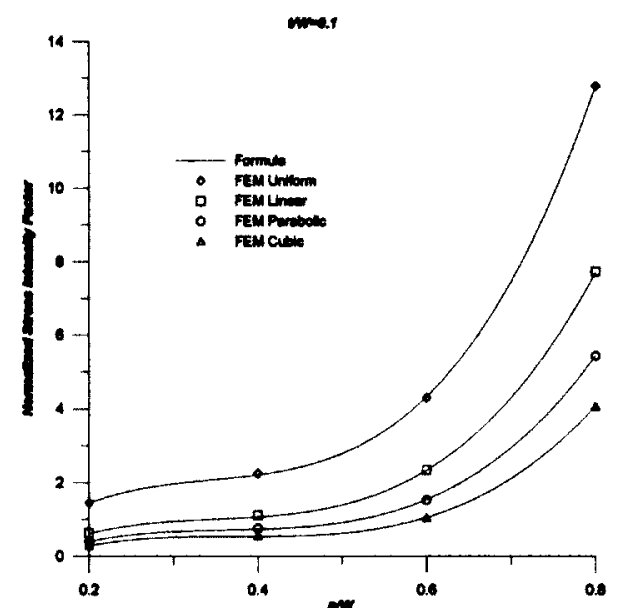

(a)

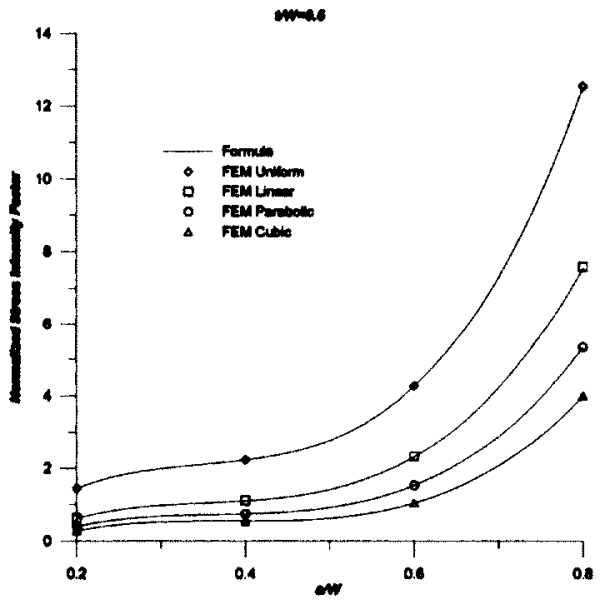

(c)

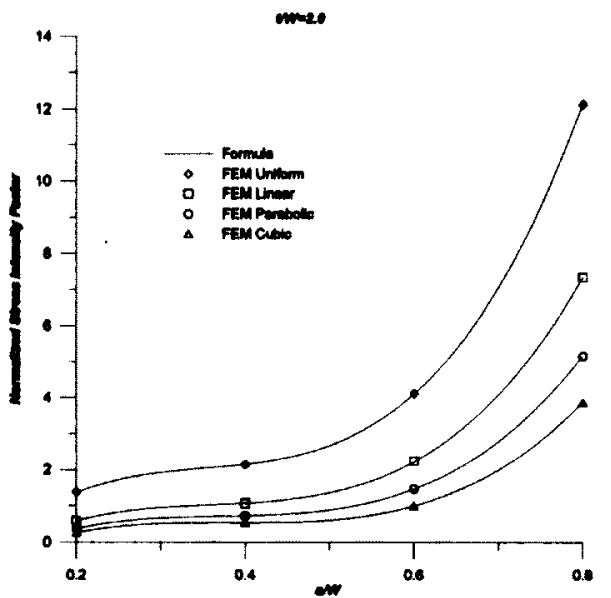

(e)

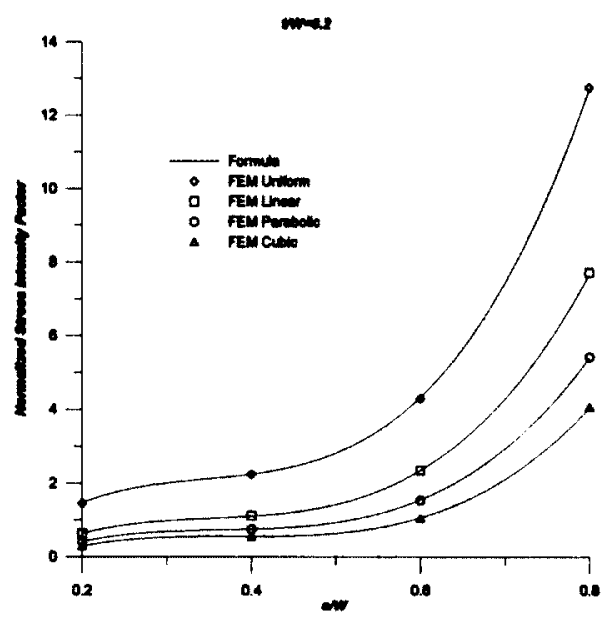

(b)

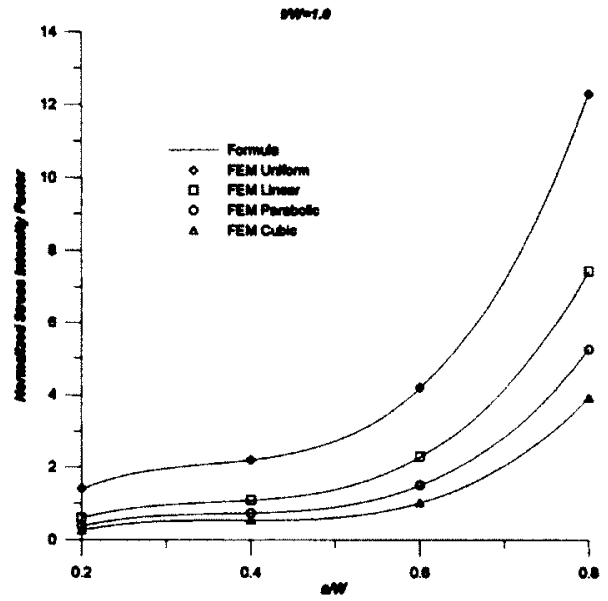

(d)

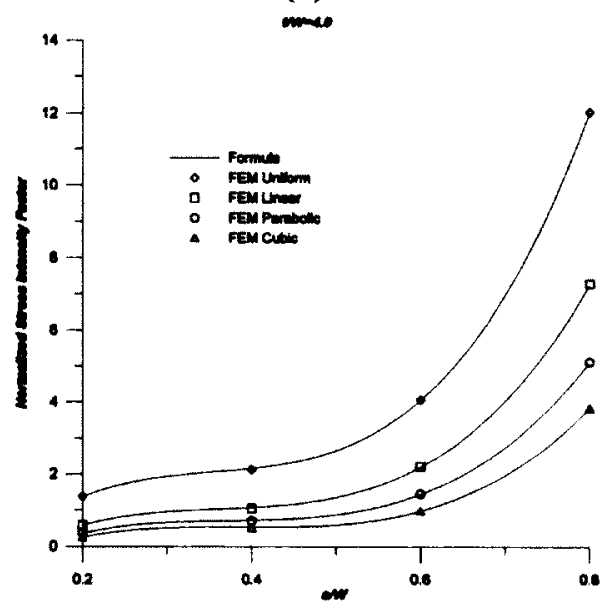

(f)

Figure 3.33 Comparison of numerical results and empirical formulae for stress intensity factor: (a) $t / W=0.1$, (b) $t / W=0.2$, (c) $t / W=0.5$, (d) $t / W=1.0$, (e) $t / W=2.0$, and (f) $t / W=4.0$ 


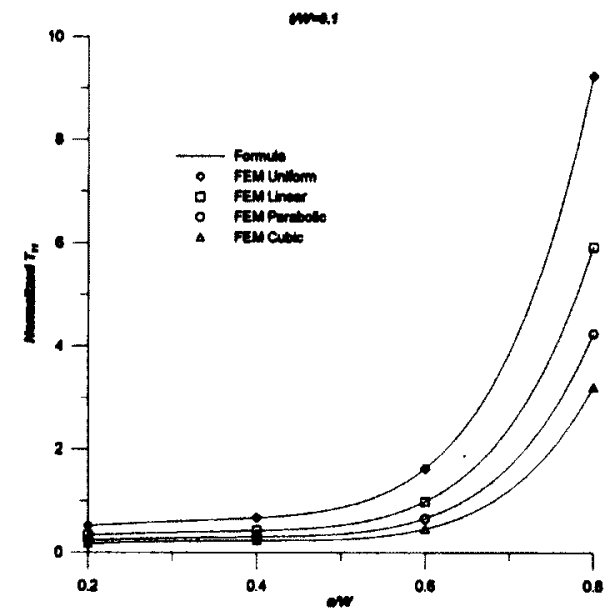

(a)

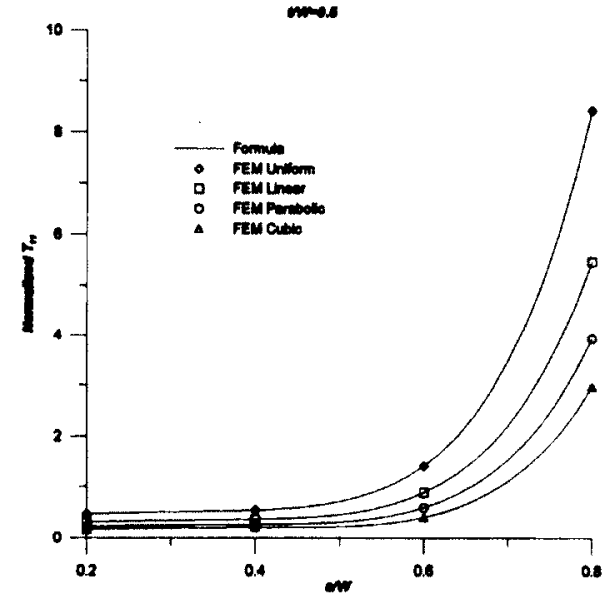

(c)

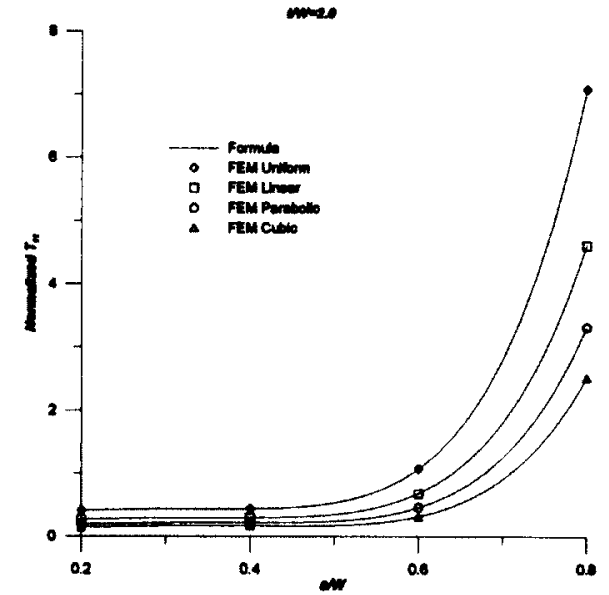

(e)

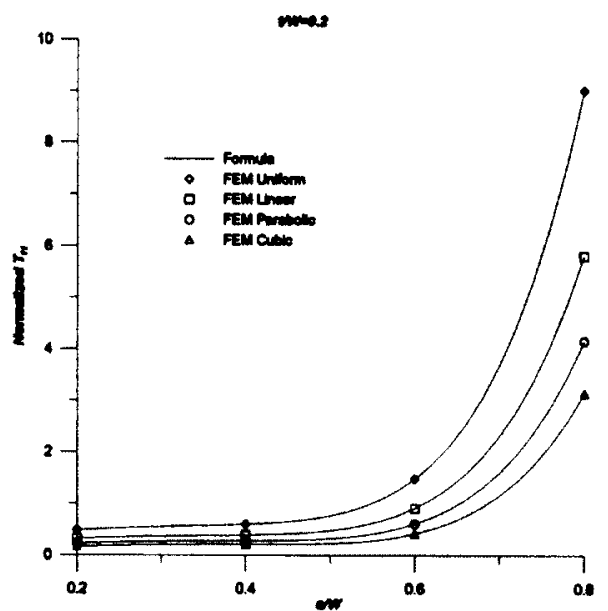

(b)

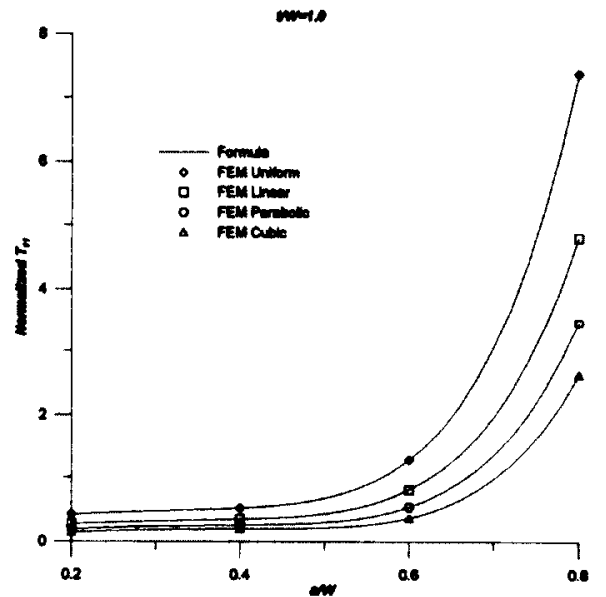

(d)

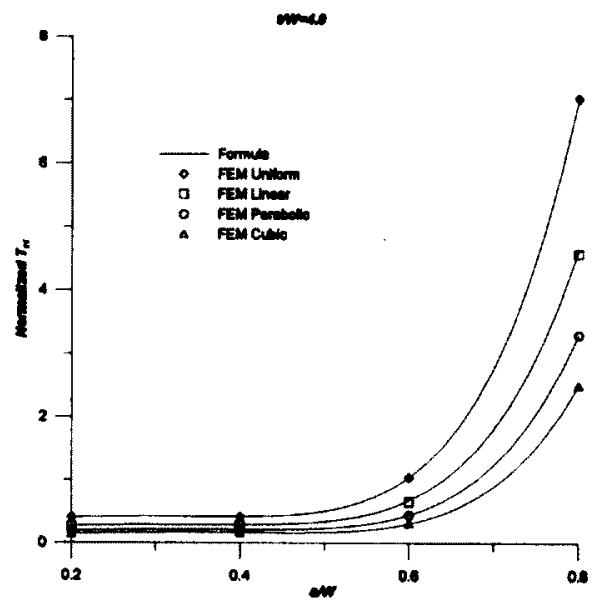

(f)

Figure 3.34 Comparison of numerical results and empirical formulae for $T_{11}$ : (a) $t / W=0.1$, (b) $t / W=0.2$, (c) $t / W=0.5$, (d) $t / W=1.0$, (e) $t / W=2.0$, and (f) $t / W=4.0$ 


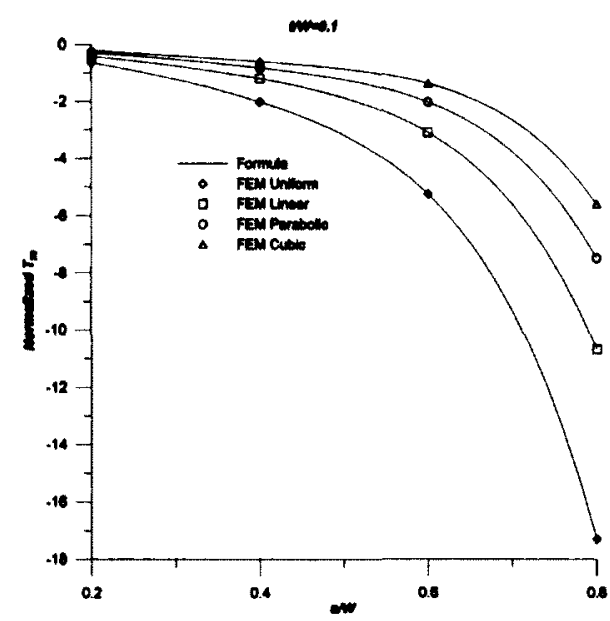

(a)

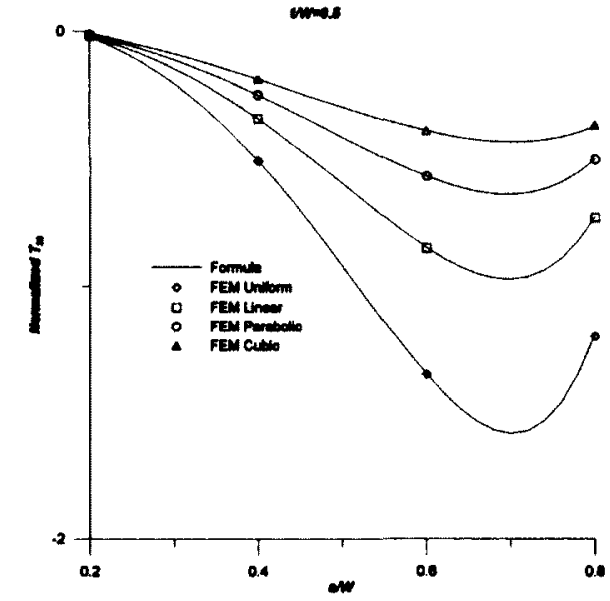

(c)

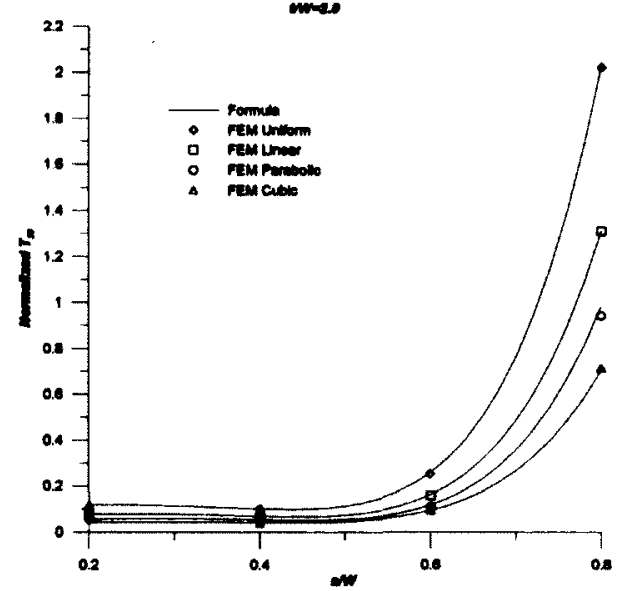

(e)

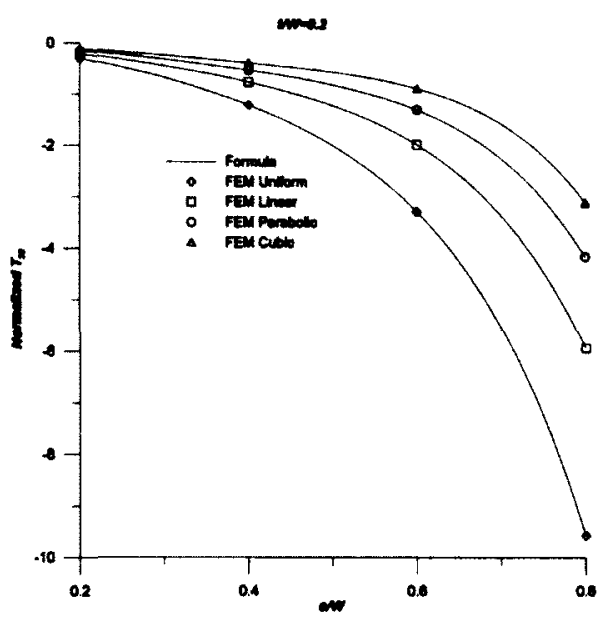

(b)

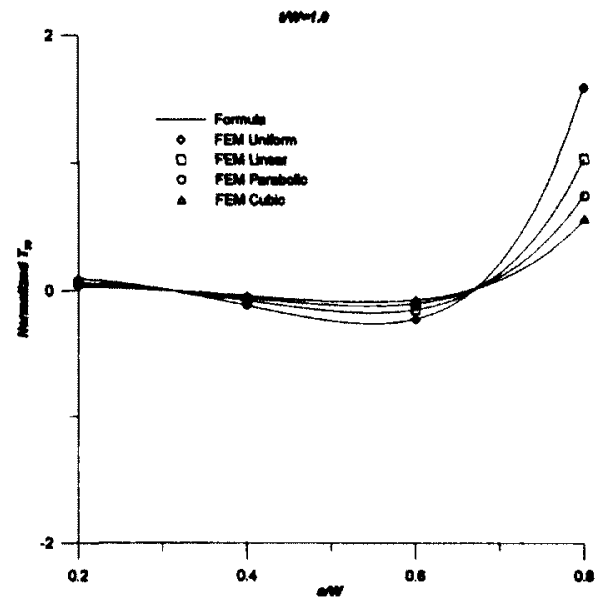

(d)

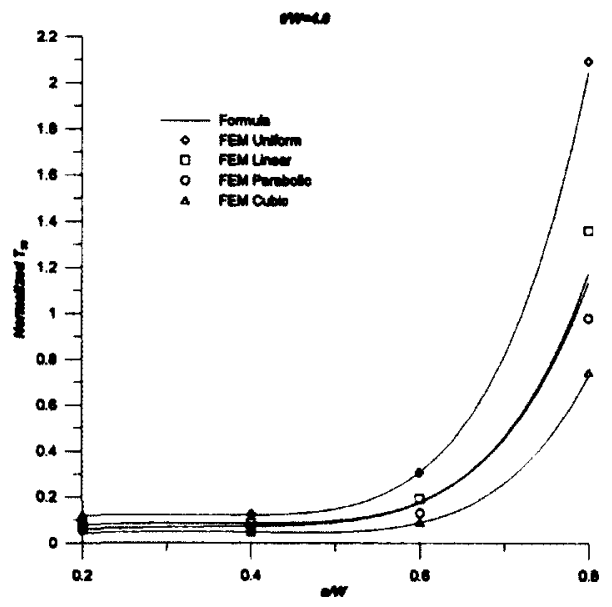

(f)

Figure 3.35 Comparison of numerical results and empirical formulae for $T_{33}$ : (a) $t / W=0.1$, (b) $t / W=0.2$, (c) $t / W=0.5$, (d) $t / W=1.0$, (e) $t / W=2.0$, and (f) $t / W=4.0$ 


\section{CHAPTER 4}

\section{VARIATIONS OF $K_{1}, T_{11}$ AND $T_{33}$ BY GEOMETRY}

In this chapter, using the finite element results obtained in Chapter $3, K_{I}, T_{11}$ and $T_{33}$ distributions of various three-dimensional single edge cracked plate specimens are studied. The influence of the plate thickness, crack depth and the variations of $K_{l}$, $T_{11}$ and $T_{33}$ along the thickness are discussed, respectively.

In order to further analyze the thinner or thicker plate effectiveness, additional $t / W=0.0025,0.025,0.05,8.0,16.0$ cases subjected to the uniform stress were computed by finite element method in ABAQUS. Additional finite element results of $K_{l}, T_{11}$ and $T_{33}$ at mid-plane for $a / W=0.2,0.4,0.6$ and 0.8 , are shown in Table 4.1-4.4, respectively. The same mesh design and method of solutions discussed in Chapter 3 are used in these calculations. In addition, the two-dimensional results of $K_{I}, T_{11}$ and $T_{33}$ for $a / W=0.2$, 0.4, 0.6 and 0.8 which taken from Sham's paper (1991) are shown in Table 4.5.

\subsection{Mid-plane $K_{1}, T_{11}$ and $T_{33}$ Varied by Geometry}

\subsubsection{Effects of plate thickness for $K_{1}, T_{11}$ and $T_{33}$}

By using the finite element analysis results from Chapter 3 and the additional thickness cases, the effect of the relative plate thickness on the three-dimensional $K_{l}$, $T_{11}$ and $T_{33}$ stresses for the crack surface subjected to the uniform stress are studied. The relative crack depth of the plate varies by $a / W=0.2,0.4,0.6$ and 0.8 , and the Poisson's ratio are fixed at $v=0.3$, respectively. The thickness variations include $t / W=0.0025,0.025,0.05,0.1,0.2,0.5,1.0,2.0,4.0,8.0$ and 16.0 . The three-dimensional 
results for mid-plane are taken from Table 3.6-3.29 and Table 4.1-4.4. And the two-dimensional results (Sham, 1991) are listed in Table 4.5.

\subsubsection{Effects of plate thickness for Stress Intensity Factor}

First, the thickness effect on $K_{I}$ is investigated. The stress intensity factor results $K_{I}$ v.s. $\log (t / a)$ are plotted in Figure $4.1-4.4$, for $a / W=0.2,0.4,0.6$ and 0.8 , respectively. It is noticed that the stress intensity factor $K_{I}$ at the mid-plane decreases when the plane is thicker. When thickness $t$ becomes large compared to the crack length $a$, the stress intensity factor trends two-dimensional solution.

$$
K_{1}^{3 D}(0)=K_{1}^{2 D} \quad \text { as } t / a \rightarrow \infty
$$

where $K_{\mathrm{I}}^{3 D}(0)$ is the stress intensity factor along the crack front, and $K_{\mathrm{I}}^{2 D}$ is the two-dimensional solution of stress intensity factor. When the $t / a \rightarrow 0$, the stress intensity factors keep increasing.

\subsubsection{Effects of plate thickness for $T_{11}$ Stress}

Normalized mid-plane value of $T_{11}$ stress as a function of plate thickness $(\log (t / a))$ in single edge through-thickness cracked plate, for $a / W=0.2,0.4,0.6$ and 0.8 , are plotted in Figure 4.5-4.8, respectively. Figure 4.5-4.8 show larger mid-plane $T_{11}$ stress in thinner plates which the crack surface subjected to the uniform stress for relative crack depth ratio $a / W=0.2,0.4,0.6$ and 0.8 , respectively. It is noticed that the normalized $T_{11}$ stress decreases and approaches the two-dimensional solution as the plate thickness increases. When $t / a \rightarrow 0, T_{11}$ stress keeps increasing.

Nakamura and Parks (1992) presented a formula to predict the mid-plane $T_{11}$ stress for SECP specimens, as described in Section 2.4.2. 
When the thickness $t$ became large compared to in-plane dimensions, $T_{11}$ can be expressed as

$$
T_{11}(z / t, v)=B^{2 D} \frac{K_{I}^{2 D}}{\sqrt{\pi a}} \equiv T_{11}^{2 D}
$$

as $t / a \rightarrow \infty$

In these thesis, only $T_{11}$ stress at mid-plane $(z / t=0)$ is discussed. The through-thickness variation of the $T_{11}$ stress distribution (Eq.2.27) where only at mid-plane can be expressed as,

$$
T_{11}(0) \cong T_{11}^{2 D}+B^{\text {thin }}(0) \frac{K_{l}(0)}{\sqrt{\pi t}}
$$

where the $T_{11}^{2 D}$ is the two-dimensional solution of $T_{11}$ stress. The $B^{\text {thin }}(0)$ represents the local "biaxiality" factor at mid-plane of a thin plate.

Then when the thickness is large, Eq. (2.26) for mid-plane can be written as,

$$
\begin{aligned}
& T_{11}(0)=B^{2 D}(0) \frac{K_{l}^{2 D}(0)}{\sqrt{\pi a}} \equiv T_{11}^{2 D} \\
& \text { as } t / a \rightarrow \infty
\end{aligned}
$$

From the Eq. (2.29), the formula of the $T_{11}$ stress distribution at mid-plane is

$$
\begin{aligned}
& T_{11}(0)=B^{\operatorname{thin}}(0) \frac{K_{\mathrm{I}}(0)}{\sqrt{\pi t}} \\
& \text { as } t / a \rightarrow 0
\end{aligned}
$$

With the use of Eq. (4.2), (4.3), and (4.4), the normalized $T_{11}(0)$ stress can be expressed as

$$
\frac{T_{11}(0)}{K_{l}(0) / \sqrt{\pi t}} \cong \sqrt{\frac{t}{a}} B^{2 D}+B^{\operatorname{thin}}(0)
$$


The first term on the right-hand side of Eq. (4.5) dominates for a thick plate, while it vanishes for a thin plate. The mid-plane values of $B^{\text {thin }}(0)$ were calculated for $a / W=0.2$, $0.4,0.6$ and 0.8 by FEM, respectively. The values of $B^{2 D}$ and $B^{\text {thin }}(0)$ are shown in Table 4.6.

For case of $a / W=0.2$, suppose $B^{\text {thin }}(0)=0.0846$ at $t / a=0.0125$, the approximate thickness-dependence of $T_{11}$ stress at mid-plane as given by Eq. (4.5) and the finite element results are shown in Table 4.7 for the relative thickness range $0.0125 \leq t / a \leq 80$ and plotted in Figure 4.9.

For case of $a / W=0.4$, suppose $B^{\text {thin }}(0)=0.0519$ at $t / a=0.00625$, the approximate thickness-dependence of $T_{11}$ stress at mid-plane as given by Eq. (4.5) and the finite element results are shown in Table 4.8 for the relative thickness range $0.00625 \leq t / a \leq 40$ and plotted in Figure 4.10.

For case of $a / W=0.6$, suppose $B^{\text {thin }}(0)=0.0526$ at $t / a=0.00427$, the approximate thickness-dependence of $T_{11}$ stress at mid-plane as given by Eq. (4.5) and the finite element results are shown in Table 4.9 for the relative thickness range $0.00427 \leq t / a \leq 27$ and plotted in Figure 4.11.

For case of $a / W=0.8$, suppose $B^{\text {thin }}(0)=0.0675$ at $t / a=0.003$, the approximate thickness-dependence of $T_{11}$ stress at mid-plane as given by Eq. (4.5) and the finite element results are shown in Table 4.10 for the relative thickness range $0.003 \leq t / a \leq 20$ and plotted in Figure 4.12.

Overall, it can be seen that Eq. (4.5) gives good approximation of $T_{11}$ stress variation for different $t / a$ ratios.

\subsubsection{Effects of plate thickness for $T_{33}$ Stress}

The normalized mid-plane $T_{33}$ stress varied by thickness $(\log (t / a))$ for $a / W=0.2,0.4,0.6$ and 0.8 are shown in Figure 4.13-4.16, respectively. It is noticed that 
the normalized $T_{33}$ stress increases and approaches the two-dimensional solution as the plate thickness increases. The three-dimensional results nearly merge with the two-dimensional solution for thicker plate. As $t / a$ decreases, the $T_{33}$ stress keeps decreasing as well.

Similar as definition of "biaxiality" parameter $B$, parameter $B_{33}$ for mid-plane is defined as

$$
B_{33}(0)=\frac{\sqrt{\pi t} T_{33}(0)}{K_{I}(0)}
$$

When the thickness is large,

$$
T_{33}(0) \equiv T_{33}^{2 D} \quad \text { as } \quad t / a \rightarrow \infty
$$

According to the prediction process of $T_{11}$ stress at mid-plane, also from considerations of superposition and dimensional analysis, as relative thickness diminished in the boundary value problem posed, the through-thickness variation of the $T_{33}$ stress distribution can be expressed as

$$
T_{33}(0) \cong T_{33}^{2 D}+B_{33}^{\text {thin }}(0) \frac{K_{l}(0)}{\sqrt{\pi t}}
$$

Here $T_{33}^{2 D}$ represents the "remote" value which applies in the plane stress field found at radii large compared to thickness $t$, but small compared to crack depth $a$. The function $B_{33}^{\text {thin }}$ represents the local biaxiality factor of a thin plane.

From the Figure $4.13-4.16$, it is noticed that the $T_{33}$ stress is still markedly decreasing at thinner plate. It is expected that there exists a $T_{33}$ stress distribution at the mid-plane,

$$
\begin{aligned}
& T_{33}(0)=B_{33}^{\text {thin }}(0) \frac{K_{I}(0)}{\sqrt{\pi t}} \\
& \text { as } \quad t / a \rightarrow 0
\end{aligned}
$$


$B_{33}^{\text {thin }}$ can be solved from the Eq. (4.9) by calculating $T_{33}(0)$ and $K_{l}(0)$ at very low $t / a$ ratio plate.

With the use of Eq. (4.6), (4.7), (4.8) and (4.9), the normalized $T_{33}(0)$ stress can be expressed as

$$
\frac{T_{33}(0)}{K_{l}(0) / \sqrt{\pi t}} \cong \sqrt{\frac{t}{a}} B_{33}^{2 D}(0)+B_{33}^{\operatorname{thin}}(0)
$$

In order to compare the results between the finite element analysis and prediction, the $B_{33}^{\text {thin }}(0)$ for $a / W=0.2,0.4,0.6$ and 0.8 with crack surface subjected to uniform load calculated by Eq. (4.9) for the thinner plane, and $T_{33}(0)$ was predicted by Eq. (4.10). The values of $T_{33}^{2 D}$ and $B_{33}^{\text {thin }}(0)$ for $a / W=0.2,0.4,0.6$ and 0.8 are shown in Table 4.11.

For the relative crack length $a / W=0.2$, using the finite element analysis results for $B_{33}(0)$ at $t / a=0.125$ to get the $B_{33}^{\text {thin }}(0)=-0.4479$. Then substitute $B_{33}^{\text {thin }}(0)$ into Eq. (4.10), the approximate thickness-dependence of $T_{33}$ stresses at mid-plane were obtained. The comparison of approximate and finite element results of $T_{33}$ stresses at mid-plane was shown in Table 4.12, and plotted in Figure 4.17.

For the relative crack depth $a / W=0.4$, using the finite element analysis results for $B_{33}(0)$ at $t / a=0.00625$ to get $B_{33}^{\text {thin }}(0)=-0.2331$. Then substitute $B_{33}^{\text {thin }}(0)$ into Eq. (4.10), the approximate thickness-dependence of $T_{33}$ stresses at mid-plane were obtained. The comparison of approximate and finite element results of $T_{33}$ stresses at mid-plane was shown in Table 4.13, and plotted in Figure 4.18.

For the relative crack depth $a / W=0.6$, using the finite element analysis results for $B_{33}(0)$ at $t / a=0.0417$ to get $B_{33}^{\text {thin }}(0)=-0.2473$. Then substitute $B_{33}^{\text {thin }}(0)$ into Eq. (4.10), the approximate thickness-dependence of $T_{33}$ stresses at mid-plane were obtained. The comparison of approximate and finite element results of $T_{33}$ stresses at mid-plane was shown in Table 4.14, and plotted in Figure 4.19. 
For the relative crack depth $a / W=0.8$, using the finite element analysis results for $B_{33}(0)$ at $t / a=0.0313$ to get $B_{33}^{\text {thin }}(0)=-0.2441$. Then substitute $B_{33}^{\text {thin }}(0)$ into Eq. (4.10), the approximate thickness-dependence of $T_{33}$ stresses at mid-plane were obtained. The comparison of approximate and finite element results of $T_{33}$ stresses at mid-plane was shown in Table 4.15, and plotted in Figure 4.20.

From these comparisons, it can be seen that Eq. (4.10) gives reasonable approximation of $T_{33}$ stress variation with different $t / a$ ratios.

\subsubsection{Variation of Crack depths}

The effects of crack depth are studied by computing mid-plane $K_{l}, T_{11}$ and $T_{33}$ values for various relative crack depths at $a / W=0.2,0.4,0.6$ and 0.8 .

The normalized stress intensity factor $K_{I}$ in the mid-plane for the relative thickness of the plate varies by $t / W=0.1,0.5,1.0$ and 4.0 , and the Poisson's ratio are fixed at $v=0.3$, as shown in Figure 4.21. The stress intensity factors for two dimensional geometries are also shown in Figure 4.21. The values of stress intensity factor all increase with increasing the crack depths whether the plane thickness is thick or thin.

The $T_{11}$ stress normalized by $\sigma_{0}$ in the mid-plane is shown in Figure 4.22. The relative thickness of the plate are varied by $t / W=0.1,0.5,1.0$ and 4.0, and the Poisson's ratio $v=0.3$. Results for two-dimensional geometries are also shown. All the cases show increasing $T_{11}$ at mid-plane for deeper crack. The three-dimensional normalized $T_{11}$ stress values are slightly higher than the corresponding two-dimensional solutions.

The $T_{33}$ stress for the same cases $(t / W=0.1,0.5,1.0$ and 4.0 , and $v=0.3)$ normalized by $\sigma_{0}$ together with two-dimensional results are shown in Figure 4.23. The $T_{33}$ stress is increasing at mid-plane for deeper crack for thicker plate $(t / W>0.5)$ and decreasing for thinner plate $(t / W=0.1)$. 


\section{2 $K_{1}, T_{11}$ and $T_{33}$ Variation along the Crack Front}

The three-dimensional stress intensity factor can be presented as two-dimensional value corrected by the thickness influence (Kwon and Sun, 2000), as discussed in section 2.4 .

$$
K_{\mathrm{I}}^{3 D}(z / t)=K_{\mathrm{I}}^{2 D} \Delta K_{3 D}(z / t)
$$

where $\Delta K_{3 D}(z / t)$ stands for the thickness correction factor. The $\Delta K_{3 D}(z / t)$ along the crack surface of $a / W=0.2,0.4,0.6$ or 0.8 are shown in Figure 4.24-4.27, respectively. And the $\Delta K_{3 D}(z / t)$ at four locations along the crack front are shown in Table 4.16-4.19 for $a / W=0.2,0.4,0.6$ or 0.8 , respectively. In all cases, the value of $\Delta K_{3 D}(z / t)$ is in the range of $0.95<\Delta K_{3 D}(z / t)<1.07$, except in the small region near the free-surfaces where the stress intensity factor must vanish at the free-surfaces $(|z / t|=0.5)$. The three-dimensional stress intensity factors are slightly different than the two-dimensional values along the most of the crack front.

The three-dimensional $T_{11}$ along the crack front various by the specimen thickness are presented in Chapter 3. It is noticed that the three-dimensional $T_{11}$ is quite different for different relative thickness ratio $t / W$. The three-dimensional $T_{11}$ decreases along the thickness at some relative thickness ratio $t / W$, but increases and then decreases along the thickness at other relative thickness ratio $t / W$. Similar as stress intensity factor, define the thickness influence factor, $\Delta T_{11_{3 D}}(z / t)$, to present the difference between the three-dimensional and two-dimensional value of $T_{11}$.

$$
T_{11}^{3 D}(z / t)=T_{11}^{2 D} \Delta T_{11_{3 D}}(z / t)
$$

The $\Delta T_{11_{3 D}}(z / t)$ along the crack front of $a / W=0.2,0.4,0.6$ or 0.8 is shown in Figure 4.28-4.31, respectively. And the values of $\Delta T_{11_{3 D}}(z / t)$ at the four locations along the 
crack front are listed in Table $4.20-4.23$ for $a / W=0.2,0.4,0.6$ or 0.8 , respectively. It shows that the values of $\Delta T_{11_{3 D}}(z / t)$ are varied by relative thickness ratio $t / W$, and deviate from the two-dimension results further for thinner plate. For example, $\Delta T_{11_{3 D}}(z)$ $t$ ) is larger than 1.6 along the most of crack front for $a / W=0.6, t / W=0.1$. That means more than $60 \%$ of error will occur if use the two-dimensional solution of $T_{11}$ to instead of the three-dimensional solution. So the two-dimension solution of $T_{11}$ is not sufficient for some three-dimensional problems.

According to the three-dimensional $T_{33}$ results obtained in Chapter 3 , it is noticed that the three-dimensional $T_{33}$ keeps decreasing along the crack front at all thickness ratio $t / W$. There is sharp dropping near the free surface. Similarly, the thickness influence factor, $\Delta T_{33}{ }_{3 D}(z / t)$, was defined to presented the difference between the three-dimensional and two-dimensional value of $T_{33}$.

$$
T_{33}^{3 D}(z / t)=T_{33}^{2 D} \Delta T_{33_{3 D}}(z / t)
$$

The $\Delta T_{33_{3 D}}(z / t)$ along the crack surface of $\mathrm{a} / \mathrm{W}=0.2,0.4,0.6$ or 0.8 is shown in Figure $4.32-4.35$, respectively. The values of $\Delta T_{33_{3 D}}(z / t)$ at four locations of the crack front for $a / W=0.2,0.4,0.6$ or 0.8 are shown in Table 4.24-4.27, respectively. It shows that the three-dimensional $T_{33}$ stresses are quite different than the two-dimensional solutions. So the three-dimensional $T_{33}$ stresses solution must be applied to solve three-dimensional questions. 
Table 4.1 Finite element result for $a / W=0.2$, subjected to uniform load, SECP

\begin{tabular}{|c|c|c|c|}
\hline$t W$ & Normalized $K_{\mathrm{I}}$ & Normalized $T_{11}$ & Normalized $T_{33}$ \\
\hline 0.0025 & 1.4729 & 1.1146 & -5.2770 \\
\hline 0.025 & 1.4579 & 0.6271 & -1.847 \\
\hline 0.05 & 1.4562 & 0.5624 & -1.142 \\
\hline 8 & 1.3649 & 0.4082 & 0.1224 \\
\hline 16 & 1.3644 & 0.4083 & 0.1226 \\
\hline
\end{tabular}

Table 4.2 Finite element result for $a / W=0.4$, subjected to uniform load, SECP

\begin{tabular}{|c|c|c|c|}
\hline$t W$ & Normalized $K_{\mathrm{I}}$ & Normalized $T_{11}$ & Normalized $T_{33}$ \\
\hline 0.0025 & 2.2847 & 1.4993 & -8.0956 \\
\hline 0.025 & 2.2484 & 0.9035 & -4.6880 \\
\hline 0.05 & 2.2472 & 0.7629 & -3.1282 \\
\hline 8 & 2.1116 & 0.4218 & 0.1269 \\
\hline 16 & 2.1082 & 0.4218 & 0.1268 \\
\hline
\end{tabular}

Table 4.3 Finite element result for $a / W=0.6$, subjected to uniform load, SECP

\begin{tabular}{|c|c|c|c|}
\hline$t W$ & Normalized $K_{\mathrm{I}}$ & Normalized $T_{11}$ & Normalized $T_{33}$ \\
\hline 0.0025 & 4.3723 & 3.5605 & -19.7328 \\
\hline 0.025 & 4.3048 & 2.1693 & -11.6595 \\
\hline 0.05 & 4.3034 & 1.8447 & -7.9499 \\
\hline 8 & 4.0462 & 1.0358 & 0.3118 \\
\hline 16 & 4.0357 & 1.0333 & 0.3111 \\
\hline
\end{tabular}


Table 4.4 Finite element result for $a / W=0.8$, subjected to uniform load, SECP

\begin{tabular}{|c|c|c|c|}
\hline$t W$ & Normalized $K_{\mathrm{I}}$ & Normalized $T_{11}$ & Normalized $T_{33}$ \\
\hline 0.0025 & 12.9629 & 15.6582 & -68.6645 \\
\hline 0.025 & 12.7781 & 10.9469 & -39.4515 \\
\hline 0.05 & 12.7781 & 9.8921 & -27.1514 \\
\hline 8 & 11.9927 & 7.0052 & 2.0996 \\
\hline 16 & 11.9699 & 6.9943 & 2.1010 \\
\hline
\end{tabular}

Table 4. 52D solution, crack subjected to uniform load, SECP, Sham (1991)

\begin{tabular}{|c|c|c|c|}
\hline$a / W$ & Normalized $K_{\mathrm{I}}$ & Normalized $T_{11}$ & Normalized $T_{33}$ \\
\hline 0.2 & 1.3650 & 0.4091 & 0.1227 \\
\hline 0.4 & 2.1083 & 0.4147 & 0.1244 \\
\hline 0.6 & 4.0254 & 1.0278 & 0.3083 \\
\hline 0.8 & 11.926 & 6.9755 & 2.0927 \\
\hline
\end{tabular}

Table 4.6 Values of $B^{2 D}$ and $B^{\text {thin }}(0)$ for $a / W=0.2,0.4,0.6$ and 0.8 subjected to uniform load on the crack surface

\begin{tabular}{|c|c|c|c|c|}
\hline $\mathrm{a} / \mathrm{W}$ & 0.2 & 0.4 & 0.6 & 0.8 \\
\hline$B^{2 D}$ & 0.2997 & 0.1967 & 0.2553 & 0.5849 \\
\hline$B^{\text {thin }}(0)$ & 0.0846 & 0.0519 & 0.0526 & 0.0675 \\
\hline t/a for $B^{\text {thin }}(0)$ & 0.0125 & 0.00625 & 0.00417 & 0.00313 \\
\hline
\end{tabular}


Table 4.7 The comparison of the approximate thickness-dependence of $T_{11}$ stress at mid-plane as given by Eq. (4.5) and the finite element results, Uniform, SECP, $a / W=0.2$

\begin{tabular}{|c|c|c|c|c|}
\hline & $\log (\mathrm{t} / \mathrm{a})$ & $\begin{array}{c}\frac{T_{11}(0)}{K_{I}(0) / \sqrt{\pi t}} \\
\text { (FEM) }\end{array}$ & $\begin{array}{c}\sqrt{\frac{t}{a}} \\
+B^{2 D} \\
(\text { thin }(0)\end{array}$ & $\begin{array}{l}\mid \frac{\text { FEM - Predict }}{\text { FEM }} \\
\times 100 \%\end{array}$ \\
\hline 0.125 & -0.9031 & 0.1521 & 0.1906 & 25.32 \\
\hline 0.25 & -0.6021 & 0.1931 & 0.2345 & 21.41 \\
\hline 0.5 & -0.3010 & 0.2503 & 0.2965 & 18.49 \\
\hline 1 & 0.0000 & 0.3315 & 0.3843 & 15.94 \\
\hline 2.5 & 0.3979 & 0.5154 & 0.5585 & 8.37 \\
\hline 5 & 0.6990 & 0.6850 & 0.7548 & 10.19 \\
\hline 10 & 1.0000 & 0.9372 & 1.0324 & 10.15 \\
\hline 20 & 1.3010 & 1.3331 & 1.4249 & 6.89 \\
\hline 40 & 1.6021 & 1.8915 & 1.9801 & 4.69 \\
\hline 80 & 1.9031 & 2.6769 & 2.7653 & 3.30 \\
\hline
\end{tabular}

Table 4.8 The comparison of the approximate thickness-dependence of $T_{11}$ stress at mid-plane as given by Eq. (4.5) and the finite element results, Uniform, SECP, $a / W=0.4$

\begin{tabular}{|c|c|c|c|c|}
\hline$t / a$ & $\log (t / a)$ & $\begin{array}{c}\frac{T_{11}(0)}{K_{I}(0) / \sqrt{\pi t}} \\
(\mathrm{FEM})\end{array}$ & $\begin{array}{l}\sqrt{\frac{t}{a}} B^{2 D} \\
+B^{\text {thin }}(0) \\
\text { (Predict) }\end{array}$ & $\begin{array}{l}\left|\frac{\text { FEM - Predict }}{\text { FEM }}\right| \\
\times 100 \%\end{array}$ \\
\hline 0.0625 & -1.2041 & 0.1005 & 0.1011 & 0.59 \\
\hline 0.125 & -0.9031 & 0.1200 & 0.1214 & 1.16 \\
\hline 0.25 & -0.6021 & 0.1479 & 0.1502 & 1.57 \\
\hline 0.5 & -0.3010 & 0.1875 & 0.1910 & 1.83 \\
\hline 1.25 & 0.0969 & 0.2745 & 0.2718 & 0.97 \\
\hline 2.5 & 0.3979 & 0.3815 & 0.3629 & 4.87 \\
\hline 5 & 0.6990 & 0.4565 & 0.4917 & 7.72 \\
\hline 10 & 1.0000 & 0.6273 & 0.6739 & 7.43 \\
\hline 20 & 1.3010 & 0.8934 & 0.9315 & 4.27 \\
\hline 40 & 1.6021 & 1.2654 & 1.2959 & 2.41 \\
\hline
\end{tabular}


Table 4.9 The comparison of the approximate thickness-dependence of $T_{11}$ stress at mid-plane as given by Eq. (4.5) and the finite element results, Uniform, SECP, $a / W=0.6$

\begin{tabular}{|c|c|c|c|c|}
\hline$t / a$ & $\log (t / a)$ & $\begin{array}{c}\frac{T_{11}(0)}{K_{l}(0) / \sqrt{\pi t}} \\
(\mathrm{FEM})\end{array}$ & $\begin{array}{l}\sqrt{\frac{t}{a}} B^{2 D} \\
+B^{\text {thin }}(0) \\
\text { (Predict) }\end{array}$ & $\begin{array}{l}\left|\frac{\text { FEM - Predict }}{\text { FEM }}\right| \\
\times 100 \%\end{array}$ \\
\hline 0.0417 & -1.3802 & 0.1029 & 0.1047 & 1.77 \\
\hline 0.0833 & -1.0792 & 0.1237 & 0.1263 & 2.05 \\
\hline 0.1667 & -0.7782 & 0.1539 & 0.1568 & 1.87 \\
\hline 0.3333 & -0.4771 & 0.1986 & 0.2000 & 0.70 \\
\hline 0.8333 & -0.0792 & 0.3013 & 0.2856 & 5.19 \\
\hline 1.6667 & 0.2218 & 0.3951 & 0.3822 & 3.27 \\
\hline 3.3333 & 0.5229 & 0.4762 & 0.5187 & 8.93 \\
\hline 6.6667 & 0.8239 & 0.6590 & 0.7118 & 8.02 \\
\hline 13.3333 & 1.1249 & 0.9347 & 0.9849 & 5.37 \\
\hline 26.6667 & 1.4260 & 1.3222 & 1.3711 & 3.69 \\
\hline
\end{tabular}

Table 4.10 The comparison of the approximate thickness-dependence of $T_{11}$ stress at mid-plane as given by Eq. (4.5) and the finite element results, Uniform, SECP, $a / W=0.8$

\begin{tabular}{|c|c|c|c|c|}
\hline$t / a$ & $\log (t / a)$ & $\begin{array}{c}\frac{T_{11}(0)}{K_{l}(0) / \sqrt{\pi t}} \\
(\mathrm{FEM})\end{array}$ & $\begin{array}{l}\sqrt{\frac{t}{a} B^{2 D}} \\
+B^{\text {thin }}(0) \\
\text { (Predict) }\end{array}$ & $\begin{array}{l}\left|\frac{\text { FEM - Predict }}{\text { FEM }}\right| \\
\times 100 \%\end{array}$ \\
\hline 0.03125 & -1.5051 & 0.1514 & 0.1709 & 11.40 \\
\hline 0.0625 & -1.2041 & 0.1935 & 0.2137 & 9.46 \\
\hline 0.125 & -0.9031 & 0.2555 & 0.2743 & 6.87 \\
\hline 0.25 & -0.6021 & 0.3532 & 0.3600 & 1.87 \\
\hline 0.625 & -0.2041 & 0.5319 & 0.5299 & 0.38 \\
\hline 1.25 & 0.0969 & 0.6703 & 0.7215 & 7.09 \\
\hline 2.5 & 0.3979 & 0.9227 & 0.9923 & 7.02 \\
\hline 5 & 0.6990 & 1.3061 & 1.3754 & 5.04 \\
\hline 10 & 1.0000 & 1.8472 & 1.9171 & 3.65 \\
\hline 20 & 1.3010 & 2.6132 & 2.6833 & 2.61 \\
\hline
\end{tabular}


Table 4.11 Values of $B_{33}{ }^{2 D}$ and $B_{33}{ }^{\text {thin }}(0)$ for $a / W=0.2,0.4,0.6$ and 0.8 subjected to uniform load on the crack surface

\begin{tabular}{|c|c|c|c|c|}
\hline $\mathrm{a} / \mathrm{W}$ & 0.2 & 0.4 & 0.6 & 0.8 \\
\hline$B_{33}^{2 D}$ & 0.0899 & 0.0590 & 0.0766 & 0.1755 \\
\hline$B_{33}^{\operatorname{thln}}(0)$ & -0.4479 & -0.2331 & -0.2473 & -0.2441 \\
\hline $\mathrm{t} / \mathrm{a}$ for $B_{33}^{\text {thln }}(0)$ & 0.125 & 0.0625 & 0.0417 & 0.0313 \\
\hline
\end{tabular}

Table 4. 12 The comparison of the approximate thickness-dependence of $T_{33}$ stress at mid-plane as given by Eq. (4.10) and the finite element results, Uniform, SECP, $a / W=0.2$

\begin{tabular}{|c|c|c|c|c|}
\hline & $\log (\mathrm{t} / \mathrm{a})$ & $\begin{array}{c}T_{33}(0) \\
K_{I}(0) / \sqrt{\pi t} \\
(\mathrm{FEM})\end{array}$ & $\begin{array}{c}\sqrt{\frac{t}{a}} B_{33}^{2 D} \\
+B_{33}^{\text {thin }}(0) \\
(\text { Predict })\end{array}$ & $\begin{array}{l}\mid \frac{\text { FEM - Predict }}{\text { FEM }} \\
\times 100 \%\end{array}$ \\
\hline 0.25 & -0.6021 & -0.3921 & -0.4029 & 2.76 \\
\hline 0.5 & -0.3010 & -0.3162 & -0.3843 & 21.56 \\
\hline 1 & 0.0000 & -0.2149 & -0.3580 & 66.59 \\
\hline 2.5 & 0.3979 & -0.0237 & -0.3057 & 1187.39 \\
\hline 5 & 0.6990 & 0.1517 & -0.2469 & 262.73 \\
\hline 10 & 1.0000 & 0.2686 & -0.1636 & 160.90 \\
\hline 20 & 1.3010 & 0.3962 & -0.0458 & 111.56 \\
\hline 40 & 1.6021 & 0.5670 & 0.1207 & 78.71 \\
\hline 80 & 1.9031 & 0.8034 & 0.3563 & 55.65 \\
\hline
\end{tabular}


Table 4. 13 The comparison of the approximate thickness-dependence of $T_{33}$ stress at mid-plane as given by Eq. (4.10) and the finite element results, Uniform, SECP, $a / W=0.4$

\begin{tabular}{|c|c|c|c|c|}
\hline$t / a$ & $\log (t / a)$ & $\begin{array}{c}\frac{T_{33}(0)}{K_{I}(0) / \sqrt{\pi t}} \\
(\mathrm{FEM})\end{array}$ & $\begin{array}{l}\sqrt{\frac{t}{a}} B_{33}^{2 D} \\
+B_{33}^{\text {thin }}(0) \\
\text { (Predict) }\end{array}$ & $\begin{array}{l}\left|\frac{\text { FEM }- \text { Predict }}{\text { FEM }}\right| \\
\times 100 \%\end{array}$ \\
\hline 0.125 & -0.9031 & -0.2201 & -0.2122 & 3.57 \\
\hline 0.25 & -0.6021 & -0.2007 & -0.2036 & 1.44 \\
\hline 0.5 & -0.3010 & -0.1726 & -0.1914 & 10.86 \\
\hline 1.25 & 0.0969 & -0.1146 & -0.1671 & 45.88 \\
\hline 2.5 & 0.3979 & -0.0377 & -0.1398 & 270.58 \\
\hline 5 & 0.6990 & 0.0464 & -0.1012 & 317.90 \\
\hline 10 & 1.0000 & 0.0829 & -0.0465 & 156.09 \\
\hline 20 & 1.3010 & 0.1202 & 0.0308 & 74.38 \\
\hline 40 & 1.6021 & 0.1701 & 0.1401 & 17.63 \\
\hline
\end{tabular}

Table 4.14 The comparison of the approximate thickness-dependence of $T_{33}$ stress at mid-plane as given by Eq. (4.10) and the finite element results, Uniform, SECP, $a / W=0.6$

\begin{tabular}{|c|c|c|c|c|}
\hline & $\log (\mathrm{t} / \mathrm{a})$ & $\frac{T_{33}(0)}{K_{I}(0) / \sqrt{\pi t}}$ & $\begin{array}{l}\sqrt{\frac{t}{a}} B_{33}^{2 D} \\
(\mathrm{FEM}) \\
+B_{33}^{\text {thin }}(0) \\
(\text { Predict })\end{array}$ & $\begin{array}{l}\mid \frac{\text { FEM - Predict }}{\text { FEM }} \\
\times 100 \%\end{array}$ \\
\hline 0.0833 & -1.0792 & -0.2385 & -0.2251 & 5.60 \\
\hline 0.1667 & -0.7782 & -0.2233 & -0.2160 & 3.28 \\
\hline 0.3333 & -0.4771 & -0.1977 & -0.2030 & 2.71 \\
\hline 0.8333 & -0.0792 & -0.1296 & -0.1773 & 36.80 \\
\hline 1.6667 & 0.2218 & -0.0319 & -0.1484 & 365.48 \\
\hline 3.3333 & 0.5229 & 0.0500 & -0.1074 & 314.76 \\
\hline 6.6667 & 0.8239 & 0.0869 & -0.0495 & 156.93 \\
\hline 13.3333 & 1.1249 & 0.1259 & 0.0324 & 74.24 \\
\hline 26.6667 & 1.4260 & 0.1780 & 0.1483 & 16.72 \\
\hline
\end{tabular}


Table 4.15 The comparison of the approximate thickness-dependence of $T_{33}$ stress at mid-plane as given by Eq. (4.10) and the finite element results, Uniform, SECP, $a / W=0.8$

\begin{tabular}{|c|c|c|c|c|}
\hline & $\log (\mathrm{t} / \mathrm{a})$ & $\begin{array}{c}\frac{T_{33}(0)}{K_{l}(0) / \sqrt{\pi t}} \\
(\mathrm{FEM})\end{array}$ & $\begin{array}{c}\sqrt{\frac{t}{a}} B_{33}^{2 D} \\
+B_{33}^{\text {thin }}(0) \\
\text { (Predict) }\end{array}$ & $\begin{array}{l}\mid \frac{\text { FEM - Predict }}{\mathrm{FEM}} \\
\times 100 \%\end{array}$ \\
\hline 0.0625 & -1.2041 & -0.2376 & -0.2002 & 15.72 \\
\hline 0.125 & -0.9031 & -0.2140 & -0.1820 & 14.94 \\
\hline 0.25 & -0.6021 & -0.1678 & -0.1563 & 6.84 \\
\hline 0.625 & -0.2041 & -0.0339 & -0.1054 & 210.49 \\
\hline 1.25 & 0.0969 & 0.0646 & -0.0479 & 174.12 \\
\hline 2.5 & 0.3979 & 0.1177 & 0.0334 & 71.66 \\
\hline 5 & 0.6990 & 0.1738 & 0.1483 & 14.69 \\
\hline 10 & 1.0000 & 0.2476 & 0.3108 & 25.53 \\
\hline 20 & 1.3010 & 0.3510 & 0.5406 & 54.01 \\
\hline
\end{tabular}

Table 4.16 The thickness correction factor $\Delta K_{3 \mathrm{D}}$ for $a / W=0.2$

\begin{tabular}{|c|c|c|c|c|c|}
\hline \multicolumn{2}{|c|}{$t / W=0.1$} & \multicolumn{2}{c|}{$t / W=0.2$} & \multicolumn{2}{c|}{$t / W=0.5$} \\
\hline$z / t$ & $\Delta K_{3 D}$ & $z / t$ & $\Delta K_{3 D}$ & $z / t$ & $\Delta K_{3 D}$ \\
\hline 0.0000 & 1.0649 & 0.0000 & 1.0618 & 0.0000 & 1.0510 \\
\hline 0.2573 & 1.0581 & 0.2525 & 1.0555 & 0.2549 & 1.0454 \\
\hline 0.4069 & 1.0356 & 0.4018 & 1.0349 & 0.4014 & 1.0262 \\
\hline 0.4555 & 1.0103 & 0.4539 & 1.0110 & 0.4581 & 1.0002 \\
\hline \multicolumn{2}{|c|}{$t / W=1.0$} & \multicolumn{2}{|c|}{$t / W=2.0$} & \multicolumn{2}{c|}{$t / W=4.0$} \\
\hline$z / t$ & $\Delta K_{3 D}$ & $z / t$ & $\Delta K_{3 D}$ & $z / t$ & $\Delta K_{3 D}$ \\
\hline 0.0000 & 1.0298 & 0.0000 & 1.0083 & 0.0000 & 1.0016 \\
\hline 0.2549 & 1.0312 & 0.2549 & 1.0152 & 0.2549 & 1.0041 \\
\hline 0.4014 & 1.0217 & 0.4014 & 1.0207 & 0.4014 & 1.0156 \\
\hline 0.4581 & 1.0009 & 0.4581 & 1.0093 & 0.4581 & 1.0175 \\
\hline
\end{tabular}


Table 4.17 The thickness correction factor $\Delta K_{3 \mathrm{D}}$ for $a / W=0.4$

\begin{tabular}{|c|c|c|c|c|c|}
\hline \multicolumn{2}{|c|}{$t / W=0.1$} & \multicolumn{2}{c|}{$t / W=0.2$} & \multicolumn{2}{c|}{$t / W=0.5$} \\
\hline$z / t$ & $\Delta K_{3 D}$ & $z / t$ & $\Delta K_{3 D}$ & $z / t$ & $\Delta K_{3 D}$ \\
\hline 0.0000 & 1.0650 & 0.0000 & 1.0628 & 0.0000 & 1.0564 \\
\hline 0.2573 & 1.0567 & 0.2525 & 1.0549 & 0.2549 & 1.0475 \\
\hline 0.4069 & 1.0315 & 0.4018 & 1.0230 & 0.4014 & 1.0211 \\
\hline 0.4555 & 1.0042 & 0.4539 & 1.0029 & 0.4581 & 0.9889 \\
\hline \multicolumn{2}{|c|}{$t / W=1.0$} & \multicolumn{2}{|c|}{$t / W=2.0$} & \multicolumn{2}{c|}{$t / W=4.0$} \\
\hline$z / t$ & $\Delta K_{3 D}$ & $z / t$ & $\Delta K_{3 D}$ & $z / t$ & $\Delta K_{3 D}$ \\
\hline 0.0000 & 1.0419 & 0.0000 & 1.0189 & 0.0000 & 1.0064 \\
\hline 0.2549 & 1.0350 & 0.2549 & 1.0208 & 0.2549 & 1.0093 \\
\hline 0.4014 & 1.0109 & 0.4014 & 1.0106 & 0.4014 & 1.0132 \\
\hline 0.4581 & 0.9786 & 0.4581 & 0.9853 & 0.4581 & 0.9996 \\
\hline
\end{tabular}

Table 4.18 The thickness correction factor $\Delta K_{3 \mathrm{D}}$ for $a / W=0.6$

\begin{tabular}{|c|c|c|c|c|c|}
\hline \multicolumn{2}{|c|}{$t / W=0.1$} & \multicolumn{2}{c|}{$t / W=0.2$} & \multicolumn{2}{c|}{$t / W=0.5$} \\
\hline$z / t$ & $\Delta K_{3 D}$ & $z / t$ & $\Delta K_{3 D}$ & $z / t$ & $\Delta K_{3 D}$ \\
\hline 0.0000 & 1.0689 & 0.0000 & 1.0680 & 0.0000 & 1.0599 \\
\hline 0.2573 & 1.0592 & 0.2525 & 1.0573 & 0.2549 & 1.0477 \\
\hline 0.4069 & 1.0306 & 0.4018 & 1.0267 & 0.4014 & 1.0129 \\
\hline 0.4555 & 1.0011 & 0.4539 & 0.9957 & 0.4581 & 0.9725 \\
\hline \multicolumn{2}{|c|}{$t / W=1.0$} & \multicolumn{2}{|c|}{$t / W=2.0$} & \multicolumn{2}{c|}{$t / W=4.0$} \\
\hline$z / t$ & $\Delta K_{3 D}$ & $z / t$ & $\Delta K_{3 D}$ & $z / t$ & $\Delta K_{3 D}$ \\
\hline 0.0000 & 1.0426 & 0.0000 & 1.0226 & 0.0000 & 1.0106 \\
\hline 0.2549 & 1.0345 & 0.2549 & 1.0222 & 0.2549 & 1.0122 \\
\hline 0.4014 & 1.0037 & 0.4014 & 1.0076 & 0.4014 & 1.0114 \\
\hline 0.4581 & 0.9627 & 0.4581 & 0.9745 & 0.4581 & 0.9934 \\
\hline
\end{tabular}


Table 4.19 The thickness correction factor $\Delta K_{3 \mathrm{D}}$ for $a / W=0.8$

\begin{tabular}{|c|c|c|c|c|c|}
\hline \multicolumn{2}{|c|}{$t / W=0.1$} & \multicolumn{2}{c|}{$t / W=0.2$} & \multicolumn{2}{c|}{$t / W=0.5$} \\
\hline$z / t$ & $\Delta K_{3 D}$ & $z / t$ & $\Delta K_{3 D}$ & $z / t$ & $\Delta K_{3 D}$ \\
\hline 0.0000 & 1.0719 & 0.0000 & 1.0696 & 0.0000 & 1.0509 \\
\hline 0.2573 & 1.0591 & 0.2525 & 1.0548 & 0.2549 & 1.0393 \\
\hline 0.4069 & 1.0236 & 0.4018 & 1.0141 & 0.4014 & 0.9990 \\
\hline 0.4555 & 0.9896 & 0.4539 & 0.9758 & 0.4581 & 0.9494 \\
\hline \multicolumn{2}{|c|}{$t / W=1.0$} & \multicolumn{2}{|c|}{$t / W=2.0$} & \multicolumn{2}{c|}{$t / W=4.0$} \\
\hline$z / t$ & $\Delta K_{3 D}$ & $z / t$ & $\Delta K_{3 D}$ & $z / t$ & $\Delta K_{3 D}$ \\
\hline 0.0000 & 1.0310 & 0.0000 & 1.0166 & 0.0000 & 1.0091 \\
\hline 0.2549 & 1.0270 & 0.2549 & 1.0166 & 0.2549 & 1.0096 \\
\hline 0.4014 & 1.0026 & 0.4014 & 1.0091 & 0.4014 & 1.0096 \\
\hline 0.4581 & 0.9587 & 0.4581 & 0.9808 & 0.4581 & 0.9989 \\
\hline
\end{tabular}

Table 4.20 The thickness correction factor $\Delta T_{113 \mathrm{D}}$ for $a / W=0.2$

\begin{tabular}{|c|c|c|c|c|c|}
\hline \multicolumn{2}{|c|}{$t / W=0.1$} & \multicolumn{2}{c|}{$t / W=0.2$} & \multicolumn{2}{c|}{$t / W=0.5$} \\
\hline$z / t$ & $\Delta T_{11_{3 D}}$ & $z / t$ & $\Delta T_{11_{3 D}}$ & $z / t$ & $\Delta T_{11_{3 D}}$ \\
\hline 0.0000 & 1.2574 & 0.0000 & 1.1743 & 0.0000 & 1.1430 \\
\hline 0.2573 & 1.2982 & 0.2525 & 1.2004 & 0.2549 & 1.1161 \\
\hline 0.4069 & 1.3652 & 0.4018 & 1.2532 & 0.4014 & 1.0951 \\
\hline 0.4555 & 1.3927 & 0.4539 & 1.2744 & 0.4581 & 1.1021 \\
\hline \multicolumn{2}{|c|}{$t / W=1.0$} & \multicolumn{2}{|c|}{$t / W=2.0$} & \multicolumn{2}{c|}{$t / W=4.0$} \\
\hline$z / t$ & $\Delta T_{11_{3 D}}$ & $z / t$ & $\Delta T_{11_{3 D}}$ & $z / t$ & $\Delta T_{113 D}$ \\
\hline 0.0000 & 1.0525 & 0.0000 & 0.9971 & 0.0000 & 0.9962 \\
\hline 0.2549 & 1.0771 & 0.2549 & 1.0264 & 0.2549 & 0.9978 \\
\hline 0.4014 & 1.0522 & 0.4014 & 1.0736 & 0.4014 & 1.0457 \\
\hline 0.4581 & 1.0412 & 0.4581 & 1.0477 & 0.4581 & 1.0712 \\
\hline
\end{tabular}


Table 4.21 The thickness correction factor $\Delta \mathrm{T}_{113 \mathrm{~s}}$ for $a / W=0.4$

\begin{tabular}{|c|c|c|c|c|c|}
\hline \multicolumn{2}{|c|}{$t / W=0.1$} & \multicolumn{2}{c|}{$t / W=0.2$} & \multicolumn{2}{c|}{$t / W=0.5$} \\
\hline$z / t$ & $\Delta T_{11_{3 D}}$ & $z / t$ & $\Delta T_{11_{3 D}}$ & $z / t$ & $\Delta T_{11_{3 D}}$ \\
\hline 0.0000 & 1.6015 & 0.0000 & 1.4331 & 0.0000 & 1.3184 \\
\hline 0.2573 & 1.6791 & 0.2525 & 1.4827 & 0.2549 & 1.3140 \\
\hline 0.4069 & 1.8244 & 0.4018 & 1.5699 & 0.4014 & 1.3271 \\
\hline 0.4555 & 1.8456 & 0.4539 & 1.6051 & 0.4581 & 1.3341 \\
\hline \multicolumn{2}{|c|}{$t / W=1.0$} & \multicolumn{2}{|c|}{$t / W=2.0$} & \multicolumn{2}{c|}{$t / W=4.0$} \\
\hline$z / t$ & $\Delta T_{11_{3 D}}$ & $z / t$ & $\Delta T_{11_{3 D}}$ & $z / t$ & $\Delta T_{1_{3} D}$ \\
\hline 0.0000 & 1.2779 & 0.0000 & 1.0575 & 0.0000 & 1.0149 \\
\hline 0.2549 & 1.2290 & 0.2549 & 1.1482 & 0.2549 & 1.0391 \\
\hline 0.4014 & 1.1412 & 0.4014 & 1.1491 & 0.4014 & 1.1675 \\
\hline 0.4581 & 1.0970 & 0.4581 & 1.0863 & 0.4581 & 1.1359 \\
\hline
\end{tabular}

Table 4.22 The thickness correction factor $\Delta T_{113 \mathrm{D}}$ for $a / W=0.6$

\begin{tabular}{|c|c|c|c|c|c|}
\hline \multicolumn{2}{|c|}{$t / W=0.1$} & \multicolumn{2}{c|}{$t / W=0.2$} & \multicolumn{2}{c|}{$t / W=0.5$} \\
\hline$z / t$ & $\Delta T_{11_{3 D}}$ & $z / t$ & $\Delta T_{11_{3 D}}$ & $z / t$ & $\Delta T_{11_{3 D}}$ \\
\hline 0.0000 & 1.5784 & 0.0000 & 1.4387 & 0.0000 & 1.3700 \\
\hline 0.2573 & 1.6443 & 0.2525 & 1.4678 & 0.2549 & 1.3271 \\
\hline 0.4069 & 1.7691 & 0.4018 & 1.5203 & 0.4014 & 1.2546 \\
\hline 0.4555 & 1.7862 & 0.4539 & 1.5407 & 0.4581 & 1.2053 \\
\hline \multicolumn{2}{|c|}{$t / W=1.0$} & \multicolumn{2}{|c|}{$t / W=2.0$} & \multicolumn{2}{c|}{$t / W=4.0$} \\
\hline$z / t$ & $\Delta T_{11_{3 D}}$ & $z / t$ & $\Delta T_{11_{3 D}}$ & $z / t$ & $\Delta T_{11_{3 D}}$ \\
\hline 0.0000 & 1.2497 & 0.0000 & 1.0446 & 0.0000 & 1.0102 \\
\hline 0.2549 & 1.2374 & 0.2549 & 1.1274 & 0.2549 & 1.0252 \\
\hline 0.4014 & 1.1262 & 0.4014 & 1.1639 & 0.4014 & 1.1473 \\
\hline 0.4581 & 1.0139 & 0.4581 & 1.0587 & 0.4581 & 1.1405 \\
\hline
\end{tabular}


Table 4. 23 The thickness correction factor $\Delta T_{113 \mathrm{D}}$ for $a / W=0.8$

\begin{tabular}{|c|c|c|c|c|c|}
\hline \multicolumn{2}{|c|}{$t / W=0.1$} & \multicolumn{2}{c|}{$t / W=0.2$} & \multicolumn{2}{c|}{$t / W=0.5$} \\
\hline$z / t$ & $\Delta T_{11_{3 D}}$ & $z / t$ & $\Delta T_{11_{3 D}}$ & $z / t$ & $\Delta T_{11_{3 D}}$ \\
\hline 0.0000 & 1.3243 & 0.0000 & 1.2919 & 0.0000 & 1.2089 \\
\hline 0.2573 & 1.3417 & 0.2525 & 1.2655 & 0.2549 & 1.1892 \\
\hline 0.4069 & 1.3789 & 0.4018 & 1.2228 & 0.4014 & 1.0767 \\
\hline 0.4555 & 1.3781 & 0.4539 & 1.1997 & 0.4581 & 0.9715 \\
\hline \multicolumn{2}{|c|}{$t / W=1.0$} & \multicolumn{2}{|c|}{$t / W=2.0$} & \multicolumn{2}{c|}{$t / W=4.0$} \\
\hline$z / t$ & $\Delta T_{11_{3 D}}$ & $z / t$ & $\Delta T_{11_{3 D}}$ & $z / t$ & $\Delta T_{11_{3 D}}$ \\
\hline 0.0000 & 1.0567 & 0.0000 & 1.0143 & 0.0000 & 1.0078 \\
\hline 0.2549 & 1.1092 & 0.2549 & 1.0289 & 0.2549 & 1.0074 \\
\hline 0.4014 & 1.1065 & 0.4014 & 1.1086 & 0.4014 & 1.0371 \\
\hline 0.4581 & 0.9978 & 0.4581 & 1.0743 & 0.4581 & 1.1064 \\
\hline
\end{tabular}

Table 4.24 The thickness correction factor $\Delta T_{333 \mathrm{D}}$ for $a / W=0.2$

\begin{tabular}{|c|c|c|c|c|c|}
\hline \multicolumn{2}{|c|}{$t / W=0.1$} & \multicolumn{2}{c|}{$t / W=0.2$} & \multicolumn{2}{c|}{$t / W=0.5$} \\
\hline$z / t$ & $\Delta T_{33_{3 D}}$ & $z / t$ & $\Delta T_{33_{3 D}}$ & $z / t$ & $\Delta T_{33_{3 D}}$ \\
\hline 0.0000 & -5.2966 & 0.0000 & -2.5383 & 0.0000 & -0.1756 \\
\hline 0.2573 & -7.1428 & 0.2525 & -3.7398 & 0.2549 & -0.9275 \\
\hline 0.4069 & -12.9485 & 0.4018 & -7.5219 & 0.4014 & -3.1734 \\
\hline 0.4555 & -19.9968 & 0.4539 & -12.3682 & 0.4581 & -6.6116 \\
\hline \multicolumn{2}{|c|}{$t / W=1.0$} & \multicolumn{2}{|c|}{$t / W=2.0$} & \multicolumn{2}{c|}{$t / W=4.0$} \\
\hline$z / t$ & $\Delta T_{33_{3} D}$ & $z / t$ & $\Delta T_{33} D$ & $z / t$ & $\Delta T_{33} D$ \\
\hline 0.0000 & 0.7772 & 0.0000 & 0.9528 & 0.0000 & 0.9871 \\
\hline 0.2549 & 0.3195 & 0.2549 & 0.8627 & 0.2549 & 0.9729 \\
\hline 0.4014 & -1.1694 & 0.4014 & 0.0704 & 0.4014 & 0.7663 \\
\hline 0.4581 & -3.5265 & 0.4581 & -1.5084 & 0.4581 & -0.1401 \\
\hline
\end{tabular}


Table 4.25 The thickness correction factor $\Delta T_{33}$ s for $a / W=0.4$

\begin{tabular}{|c|c|c|c|c|c|}
\hline \multicolumn{2}{|c|}{$t / W=0.1$} & \multicolumn{2}{|c|}{$t / W=0.2$} & \multicolumn{2}{|c|}{$t / W=0.5$} \\
\hline$z / t$ & $\Delta T_{33_{3 D}}$ & $z / t$ & $\Delta T_{33_{3 D}}$ & $z / t$ & $\Delta T_{33_{3 D}}$ \\
\hline 0.0000 & -16.1999 & 0.0000 & -9.8330 & 0.0000 & -4.1023 \\
\hline 0.2573 & -20.2609 & 0.2525 & -12.5909 & 0.2549 & -5.8980 \\
\hline 0.4069 & -33.1427 & 0.4018 & -20.9809 & 0.4014 & -11.1356 \\
\hline 0.4555 & -48.3556 & 0.4539 & -31.5763 & 0.4581 & -18.7680 \\
\hline \multicolumn{2}{|c|}{$t / W=1.0$} & \multicolumn{2}{|c|}{$t / W=2.0$} & \multicolumn{2}{|c|}{$t / W=4.0$} \\
\hline$z / t$ & $\Delta T_{33_{3 D}}$ & $z / t$ & $\Delta T_{33_{3 D}}$ & $z / t$ & $\Delta T_{33_{3 D}}$ \\
\hline 0.0000 & -0.9421 & 0.0000 & 0.8016 & 0.0000 & 0.9998 \\
\hline 0.2549 & -2.3454 & 0.2549 & -0.0548 & 0.2549 & 0.8926 \\
\hline 0.4014 & -6.1929 & 0.4014 & -2.8598 & 0.4014 & -0.5366 \\
\hline 0.4581 & -11.6437 & 0.4581 & -6.7835 & 0.4581 & -3.3260 \\
\hline
\end{tabular}

Table 4.26 The thickness correction factor $\Delta T_{333 \mathrm{D}}$ for $a W=0.6$

\begin{tabular}{|c|c|c|c|c|c|}
\hline \multicolumn{2}{|c|}{$t / W=0.1$} & \multicolumn{2}{c|}{$t / W=0.2$} & \multicolumn{2}{c|}{$t / W=0.5$} \\
\hline$z / t$ & $\Delta T_{33_{3 D}}$ & $z / t$ & $\Delta T_{33_{3 D}}$ & $z / t$ & $\Delta T_{33_{3 D}}$ \\
\hline 0.0000 & -17.0708 & 0.0000 & -10.6760 & 0.0000 & -4.3943 \\
\hline 0.2573 & -21.0026 & 0.2525 & -13.4199 & 0.2549 & -6.3942 \\
\hline 0.4069 & -33.2982 & 0.4018 & -21.5420 & 0.4014 & -11.7755 \\
\hline 0.4555 & -47.6661 & 0.4539 & -31.5682 & 0.4581 & -19.0998 \\
\hline \multicolumn{2}{|c|}{$t / W=1.0$} & \multicolumn{2}{|c|}{$t / W=2.0$} & \multicolumn{2}{c|}{$t / W=4.0$} \\
\hline$z / t$ & $\Delta T_{33} D$ & $z / t$ & $\Delta T_{33} D$ & $z / t$ & $\Delta T_{33}{ }_{3 D}$ \\
\hline 0.0000 & -0.7515 & 0.0000 & 0.8179 & 0.0000 & 0.9936 \\
\hline 0.2549 & -2.4036 & 0.2549 & 0.0325 & 0.2549 & 0.8943 \\
\hline 0.4014 & -6.7266 & 0.4014 & -3.0870 & 0.4014 & -0.4794 \\
\hline 0.4581 & -12.2303 & 0.4581 & -7.3872 & 0.4581 & -3.6567 \\
\hline
\end{tabular}


Table 4.27 The thickness correction factor $\Delta T_{33 \text { D }}$ for $a / W=0.8$

\begin{tabular}{|c|c|c|c|c|c|}
\hline \multicolumn{2}{|c|}{$t / W=0.1$} & \multicolumn{2}{c|}{$t / W=0.2$} & \multicolumn{2}{c|}{$t / W=0.5$} \\
\hline$z / t$ & $\Delta T_{33_{3 D}}$ & $z / t$ & $\Delta T_{33_{3 D}}$ & $z / t$ & $\Delta T_{33_{3 D}}$ \\
\hline 0.0000 & -8.2683 & 0.0000 & -4.5748 & 0.0000 & -0.5748 \\
\hline 0.2573 & -10.3662 & 0.2525 & -6.1430 & 0.2549 & -1.8996 \\
\hline 0.4069 & -16.7165 & 0.4018 & -10.5036 & 0.4014 & -5.2039 \\
\hline 0.4555 & -23.9532 & 0.4539 & -15.5995 & 0.4581 & -9.1780 \\
\hline \multicolumn{2}{|c|}{$t / W=1.0$} & \multicolumn{2}{|c|}{$t / W=2.0$} & \multicolumn{2}{c|}{$t / W=4.0$} \\
\hline$z / t$ & $\Delta T_{33_{3 D}}$ & $z / t$ & $\Delta T_{33_{3 D}}$ & $z / t$ & $\Delta T_{33_{3 D}}$ \\
\hline 0.0000 & 0.7594 & 0.0000 & 0.9647 & 0.0000 & 0.9995 \\
\hline 0.2549 & 0.0931 & 0.2549 & 0.8515 & 0.2549 & 0.9775 \\
\hline 0.4014 & -2.4024 & 0.4014 & -0.3450 & 0.4014 & 0.7312 \\
\hline 0.4581 & -5.6794 & 0.4581 & -2.9044 & 0.4581 & -0.6816 \\
\hline
\end{tabular}




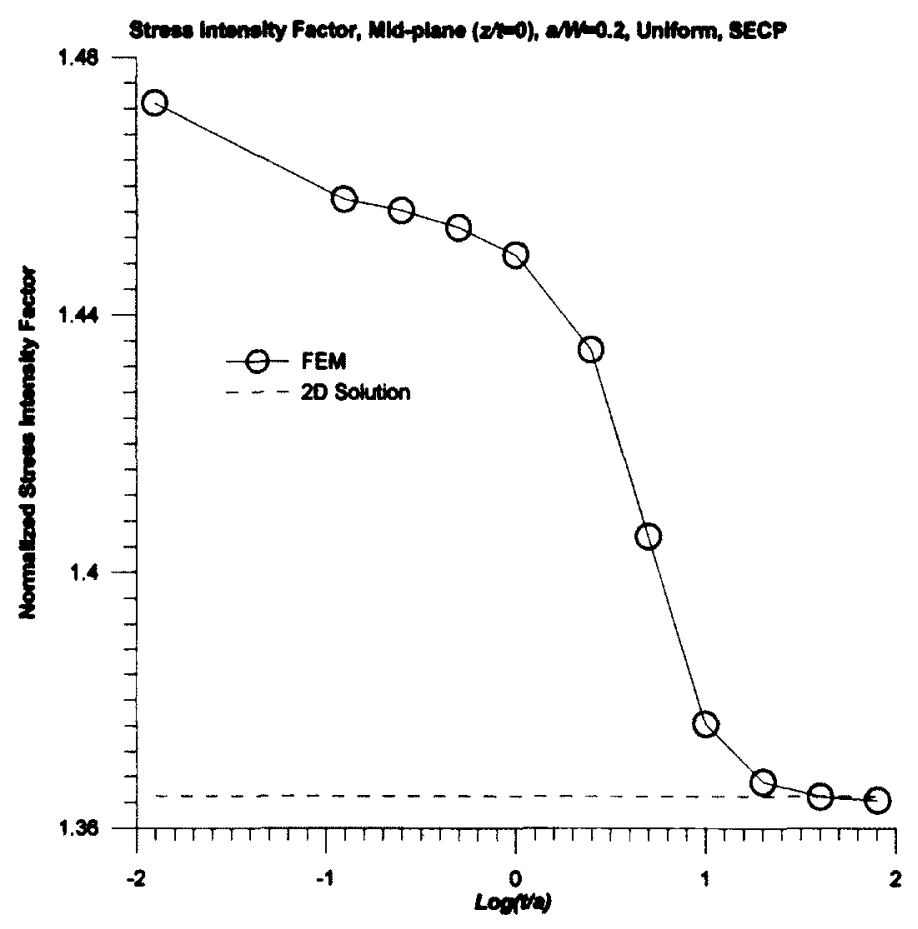

Figure 4.1 Mid-plane value of $K_{1}$ as a function of plate thickness in SECP, $a / W=0.2$

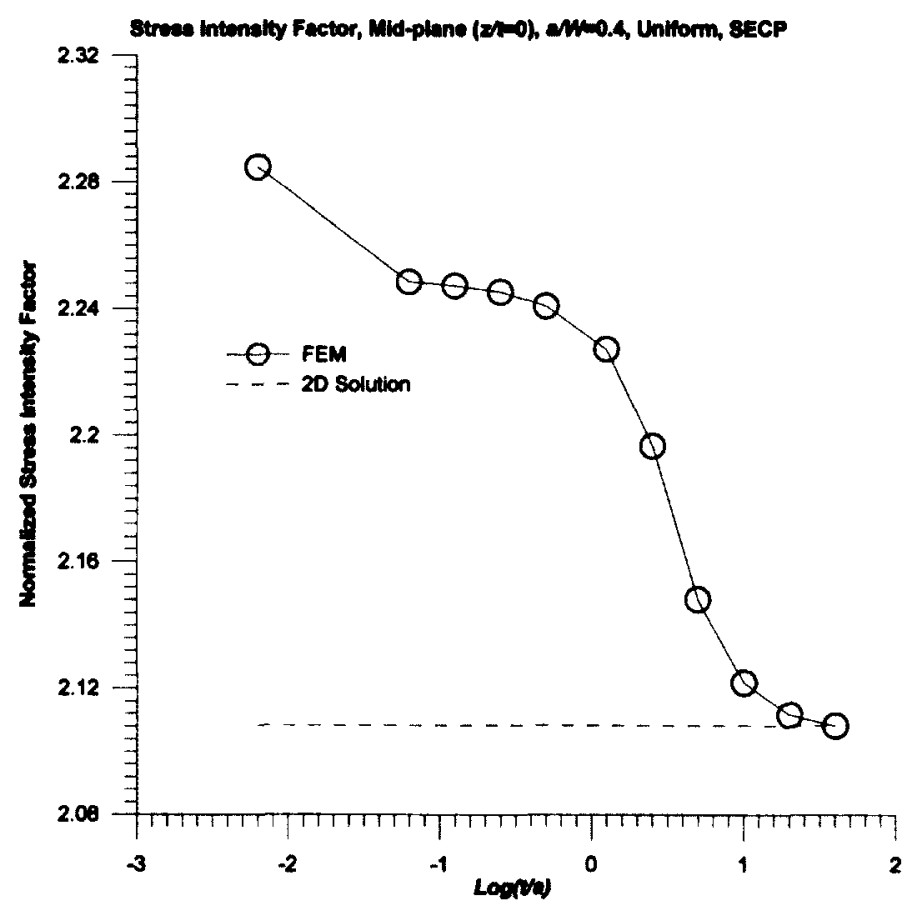

Figure 4.2 Mid-plane value of $K_{I}$ as a function of plate thickness in SECP, $a / W=0.4$ 


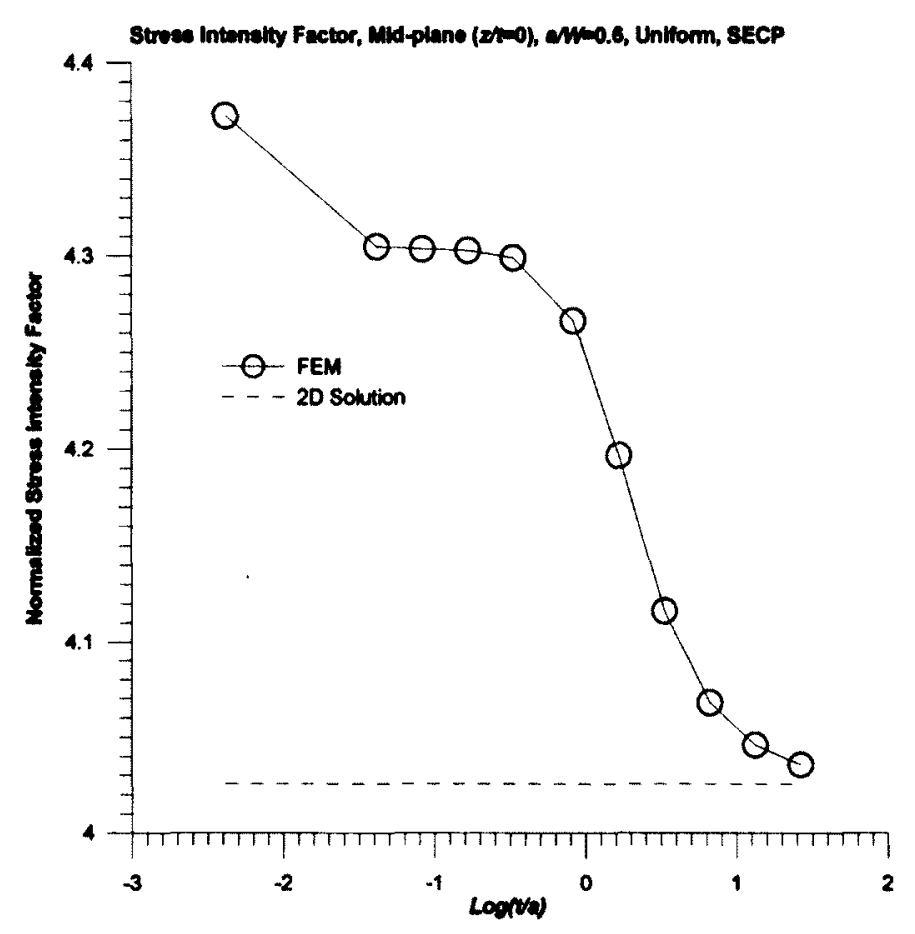

Figure 4.3 Mid-plane value of $K_{I}$ as a function of plate thickness in SECP, $a / W=0.6$

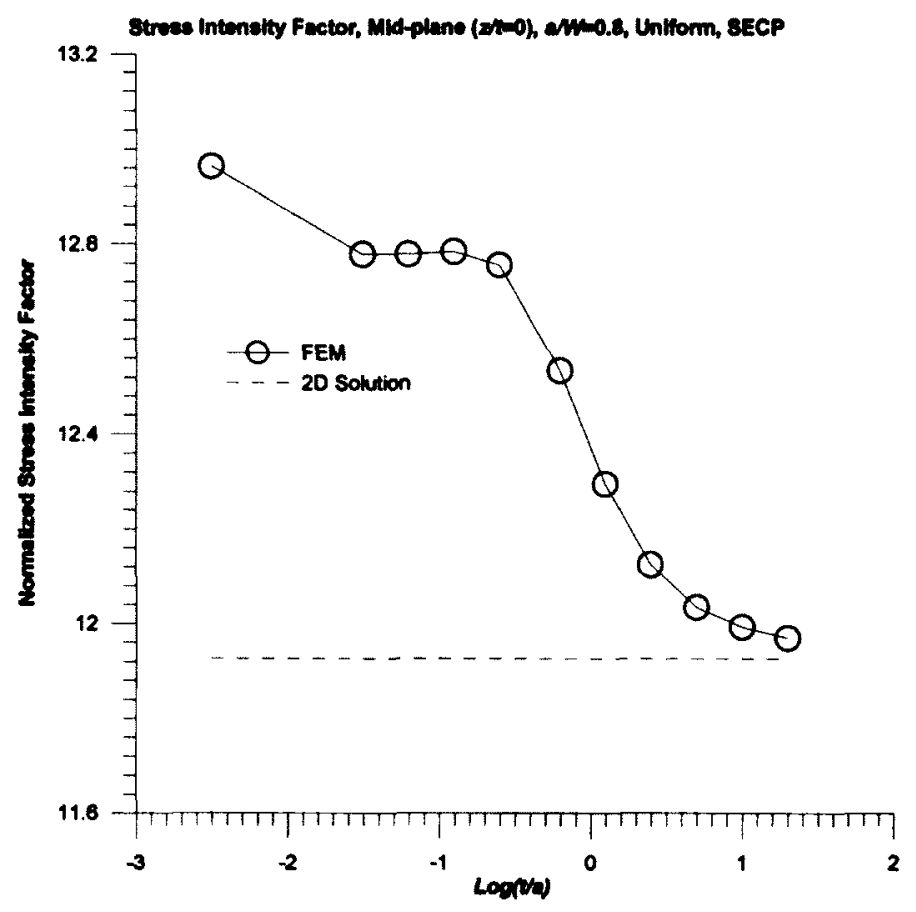

Figure 4.4 Mid-plane value of $K_{\mathrm{I}}$ as a function of plate thickness in SECP, $a / W=0.8$ 


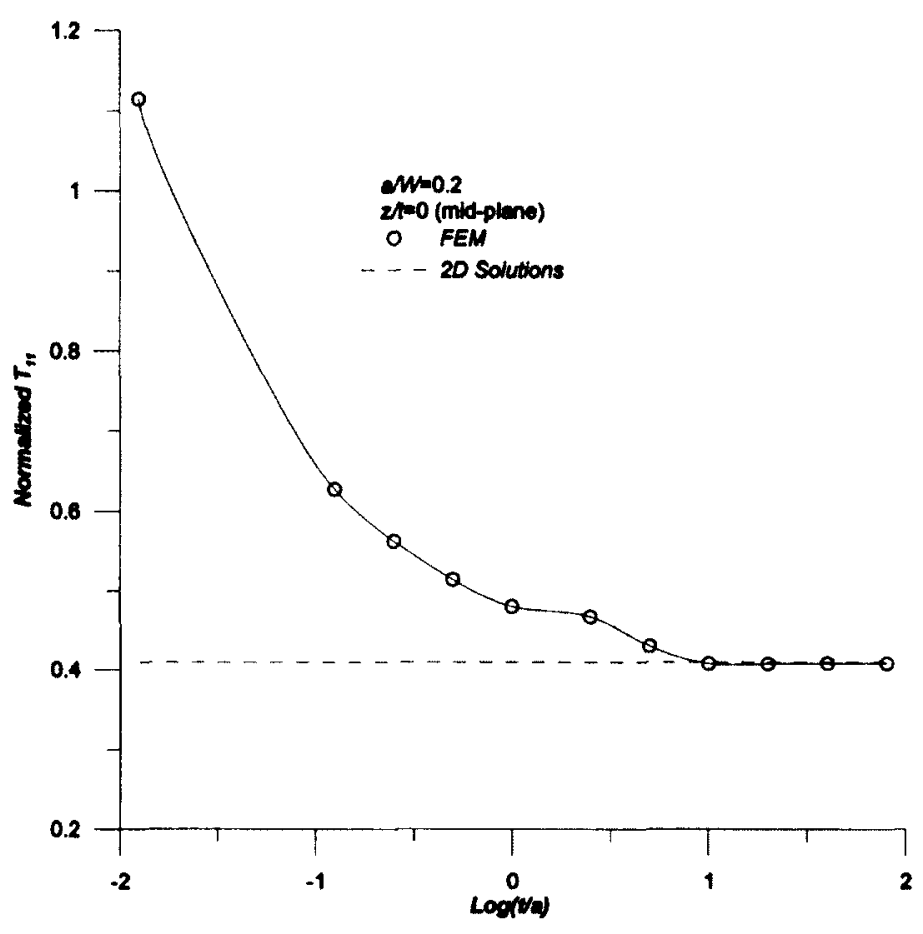

Figure 4.5 Mid-plane value of $T_{11}$ stress as a function of plate thickness in SECP, $a / W=0.2$

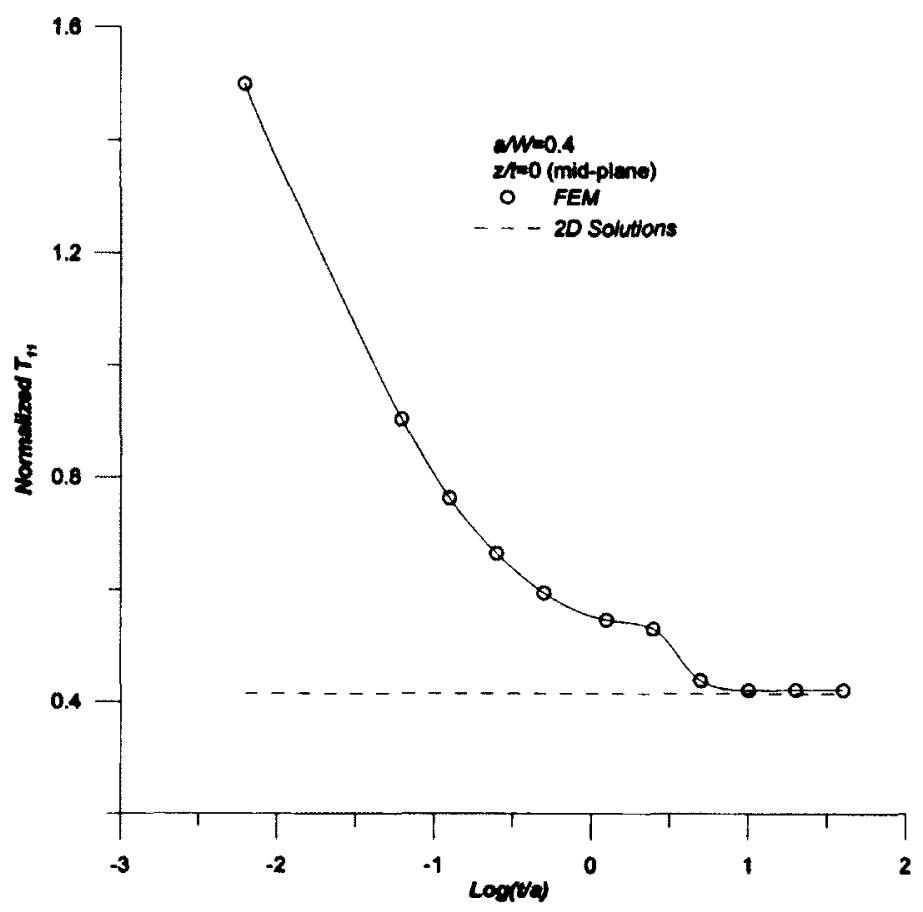

Figure 4.6 Mid-plane value of $T_{11}$ stress as a function of plate thickness in SECP, $a / W=0.4$ 


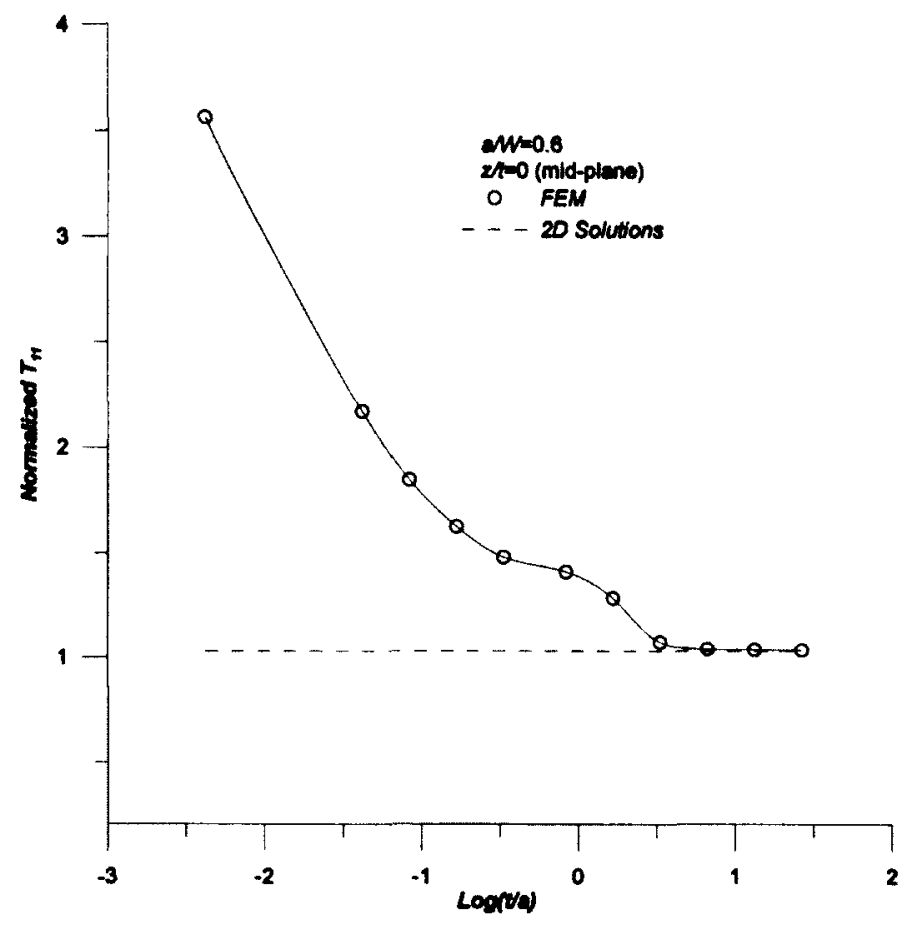

Figure 4.7 Mid-plane value of $T_{11}$ stress as a function of plate thickness in SECP, $a / W=0.6$

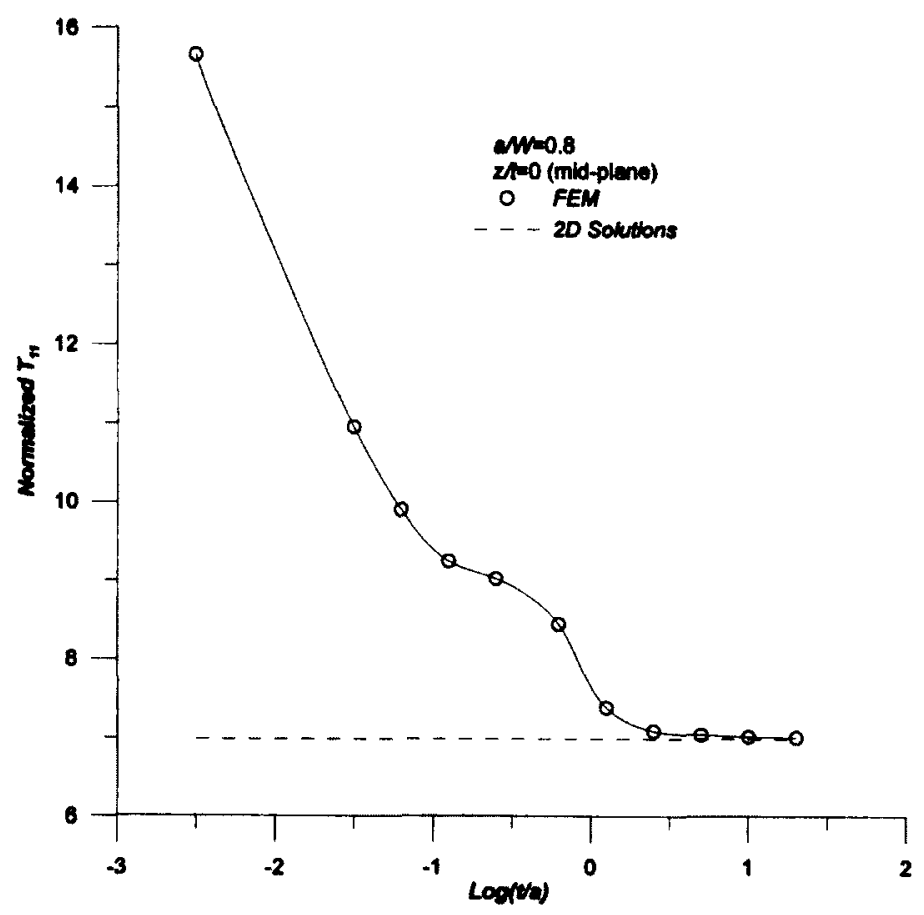

Figure 4.8 Mid-plane value of $T_{11}$ stress as a function of plate thickness in SECP, $a / W=0.8$ 


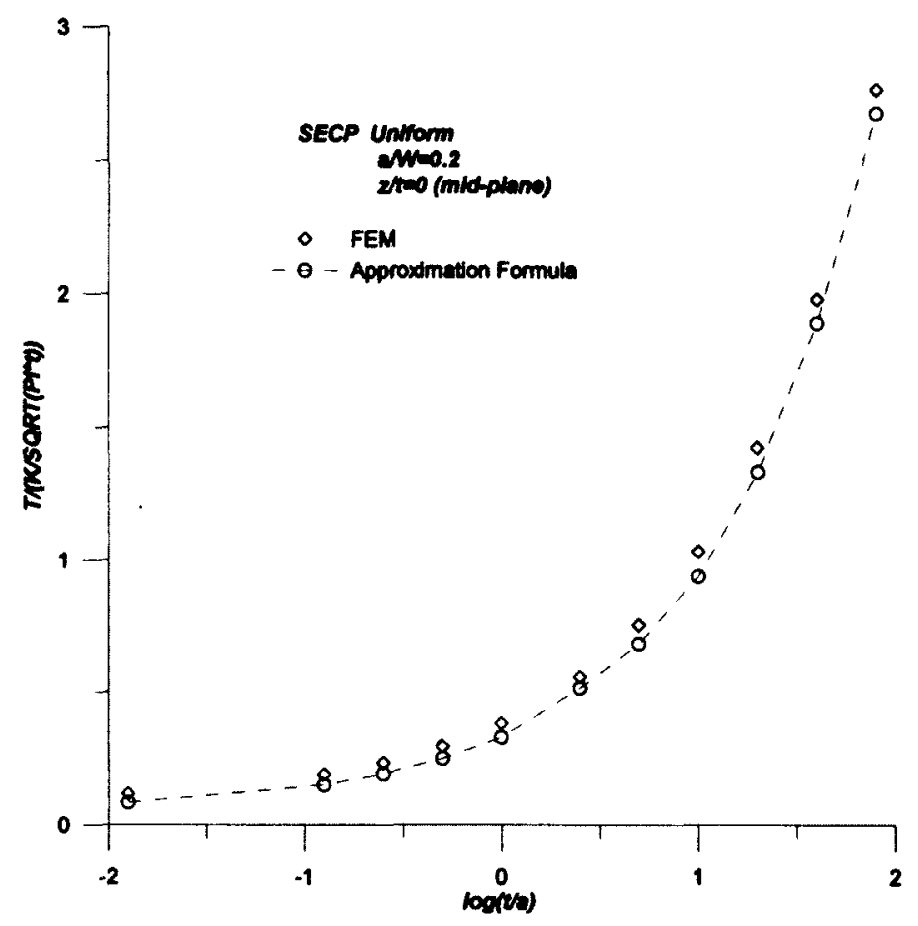

Figure 4.9 Comparison of approximation formula and FEM for $T_{11}, a / W=0.2$

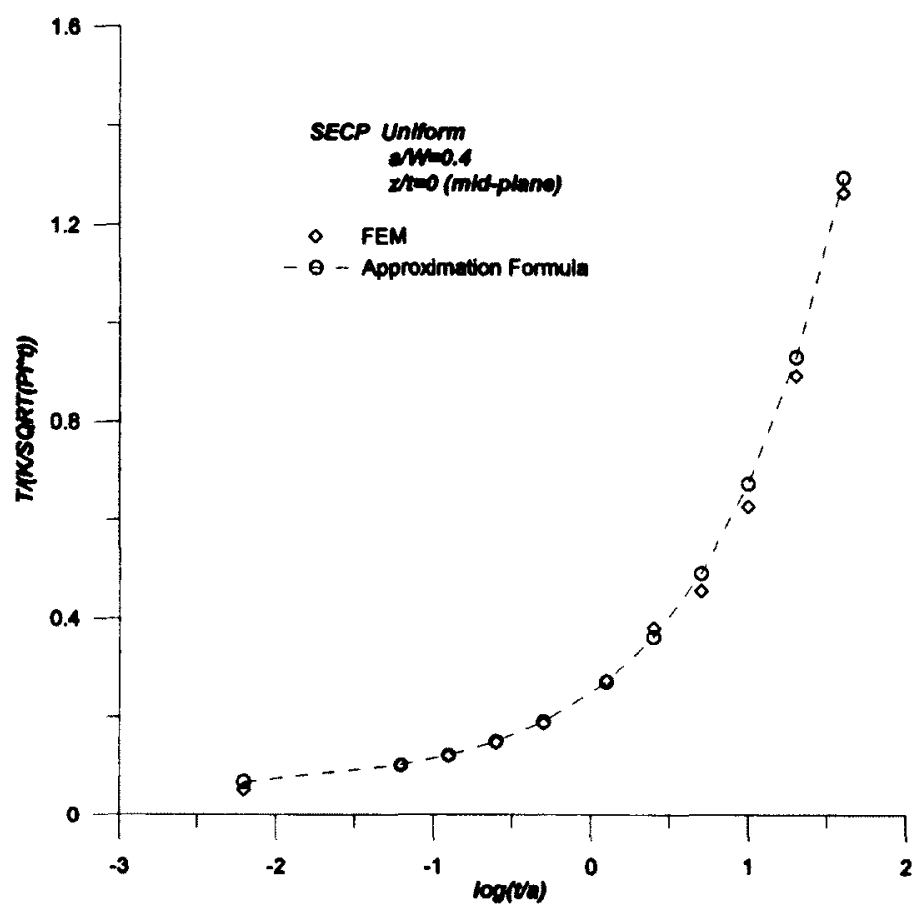

Figure 4.10 Comparison of approximation formula and FEM for $T_{11}, a / W=0.4$ 


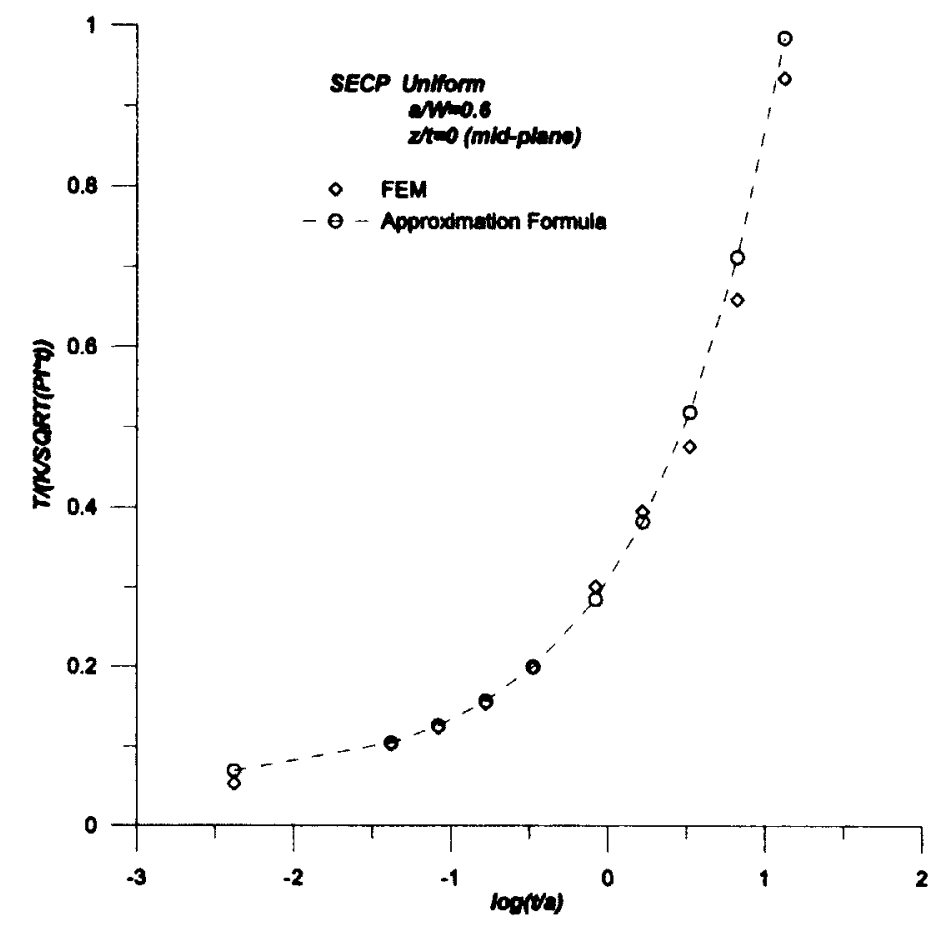

Figure 4.11 Comparison of approximation formula and FEM for $T_{11}, a / W=0.6$

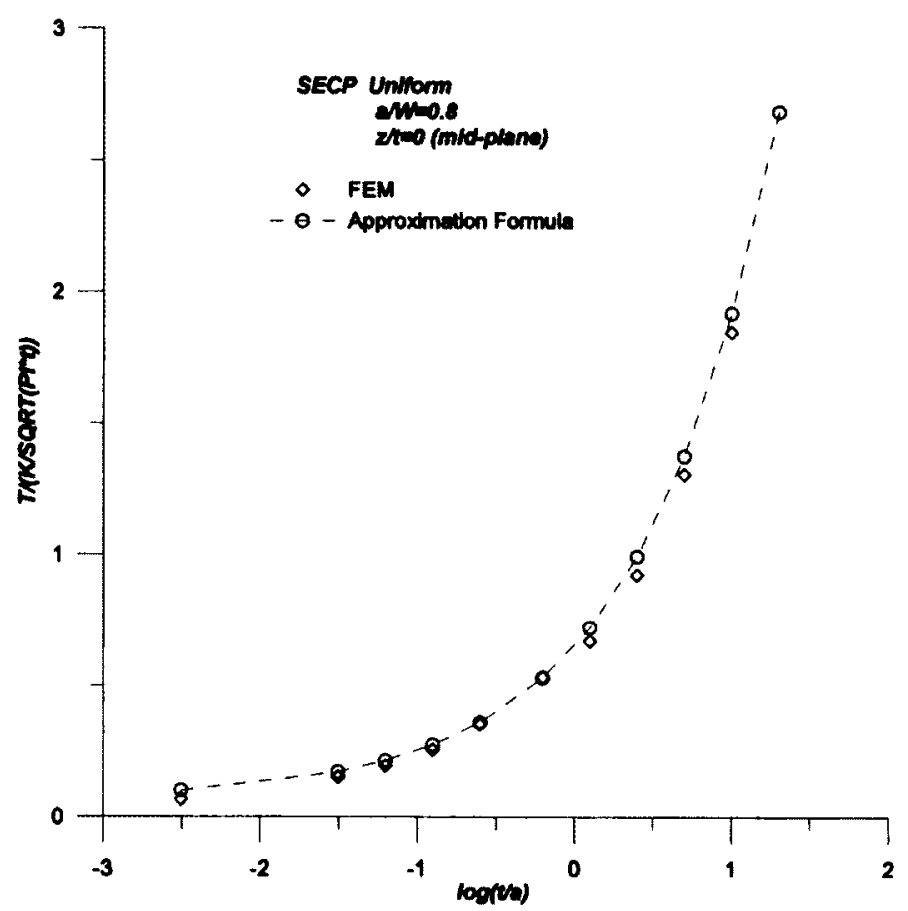

Figure 4.12 Comparison of approximation formula and FEM for $T_{11}, a / W=0.8$ 


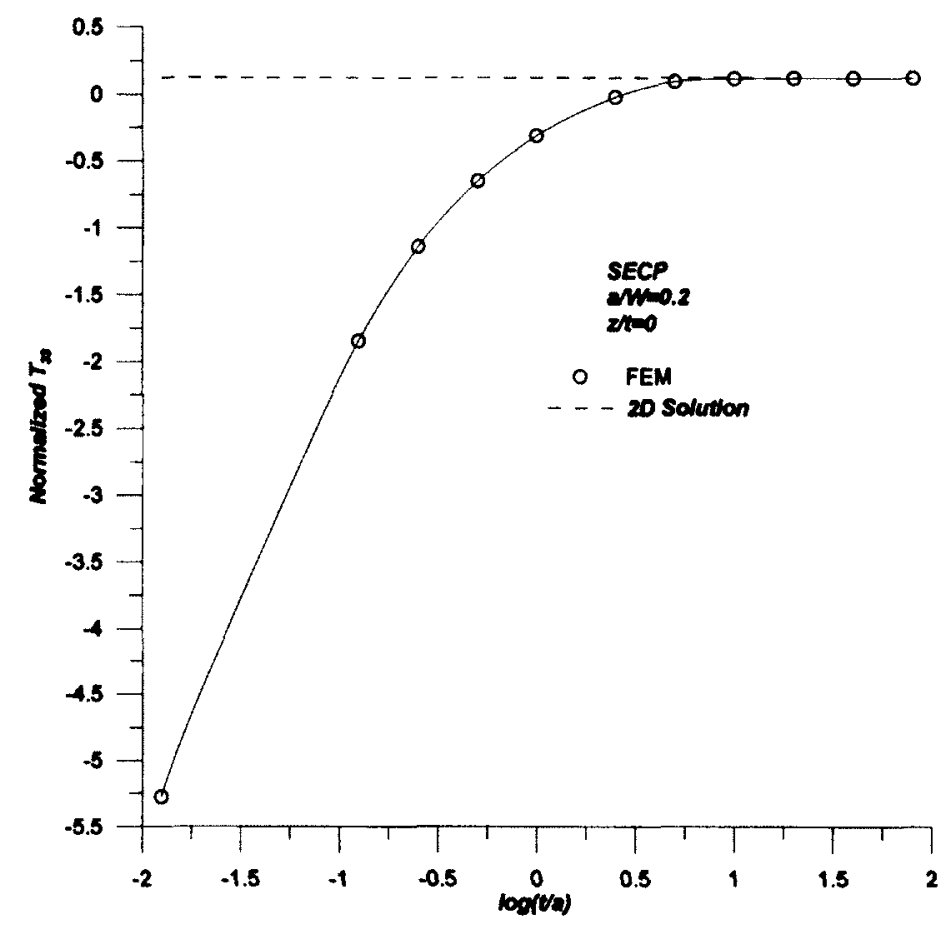

Figure 4.13 Mid-plane value of $T_{33}$ stress as a function of plate thickness in SECP, $a / W=0.2$

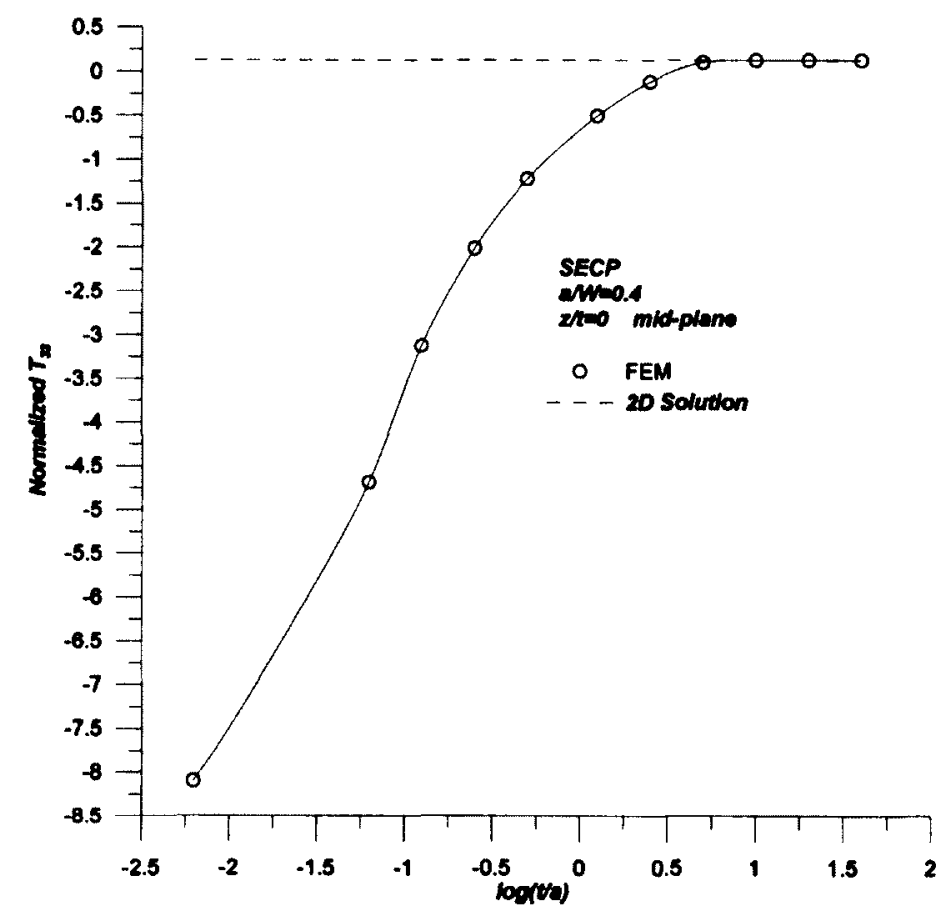

Figure 4.14 Mid-plane value of $T_{33}$ stress as a function of plate thickness in SECP, $a / W=0.4$ 


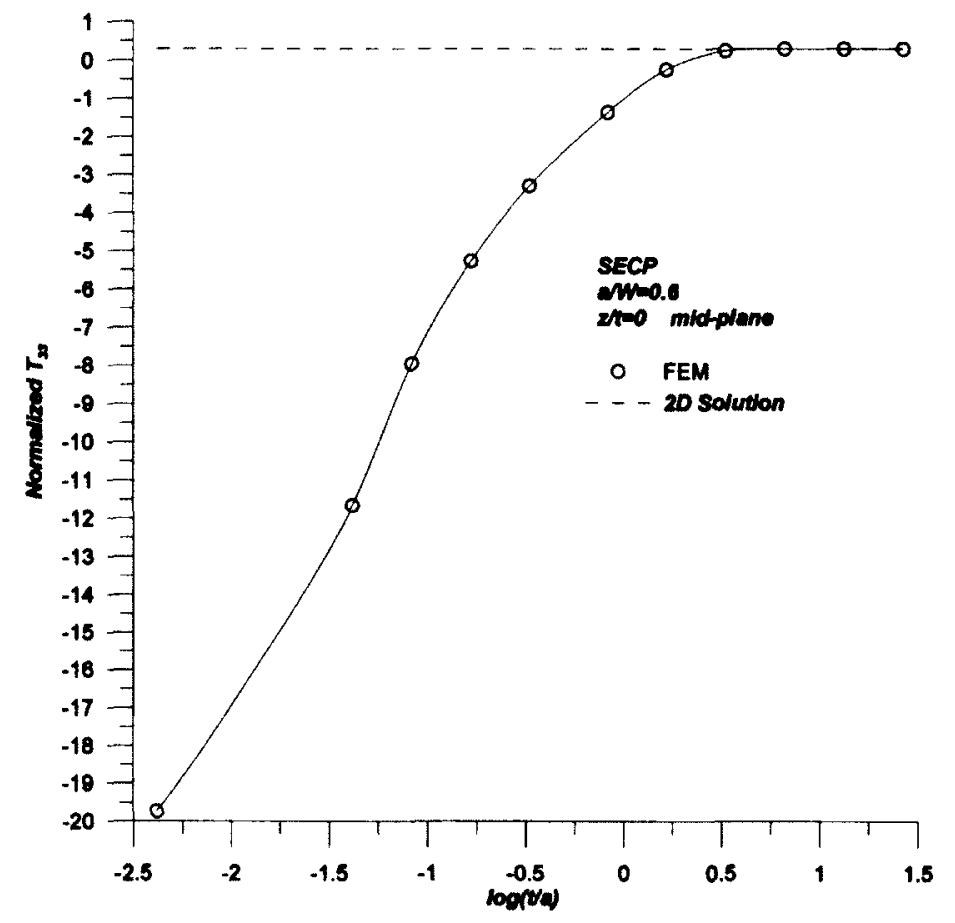

Figure 4.15 Mid-plane value of $T_{33}$ stress as a function of plate thickness in SECP, $a / W=0.6$

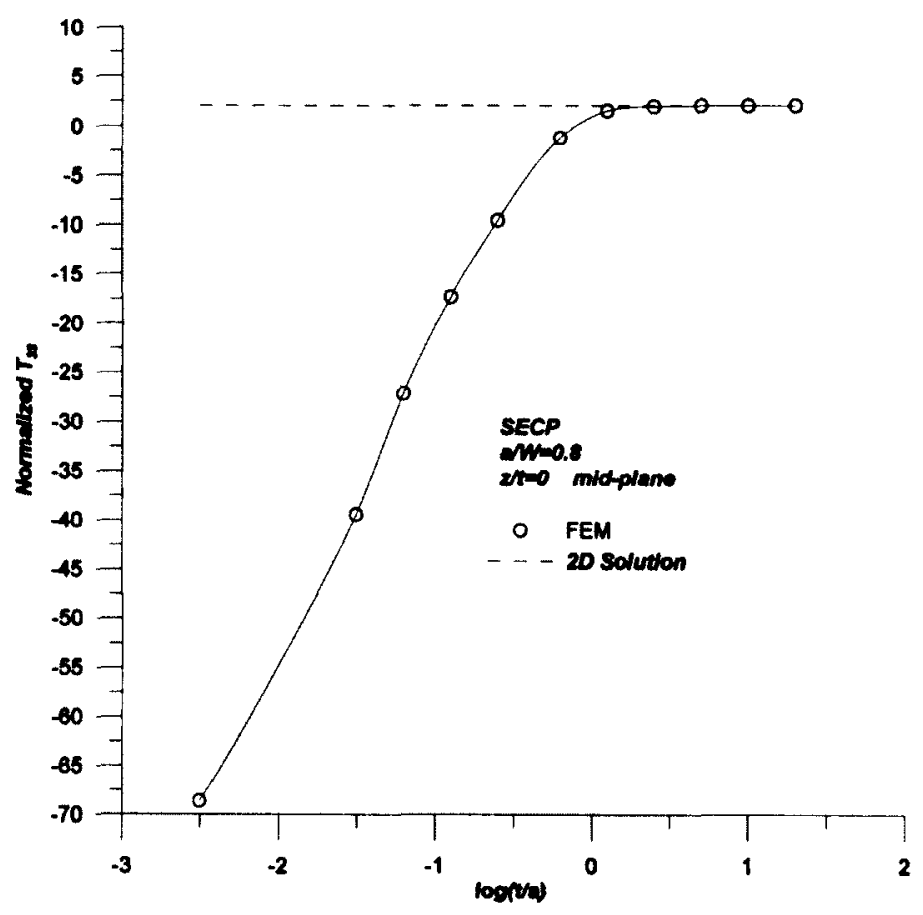

Figure 4.16 Mid-plane value of $T_{33}$ stress as a function of plate thickness in SECP, $a / W=0.8$ 


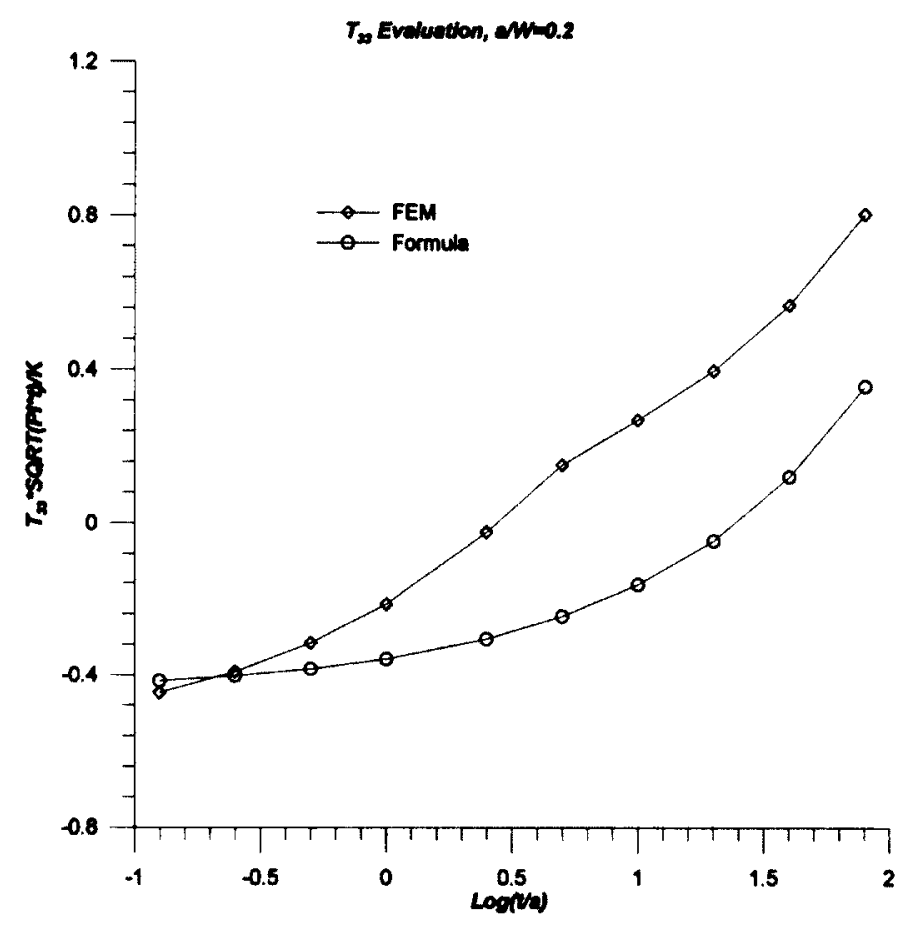

Figure 4.17 Comparison of approximation formula and FEM for $T_{33}, a / W=0.2$

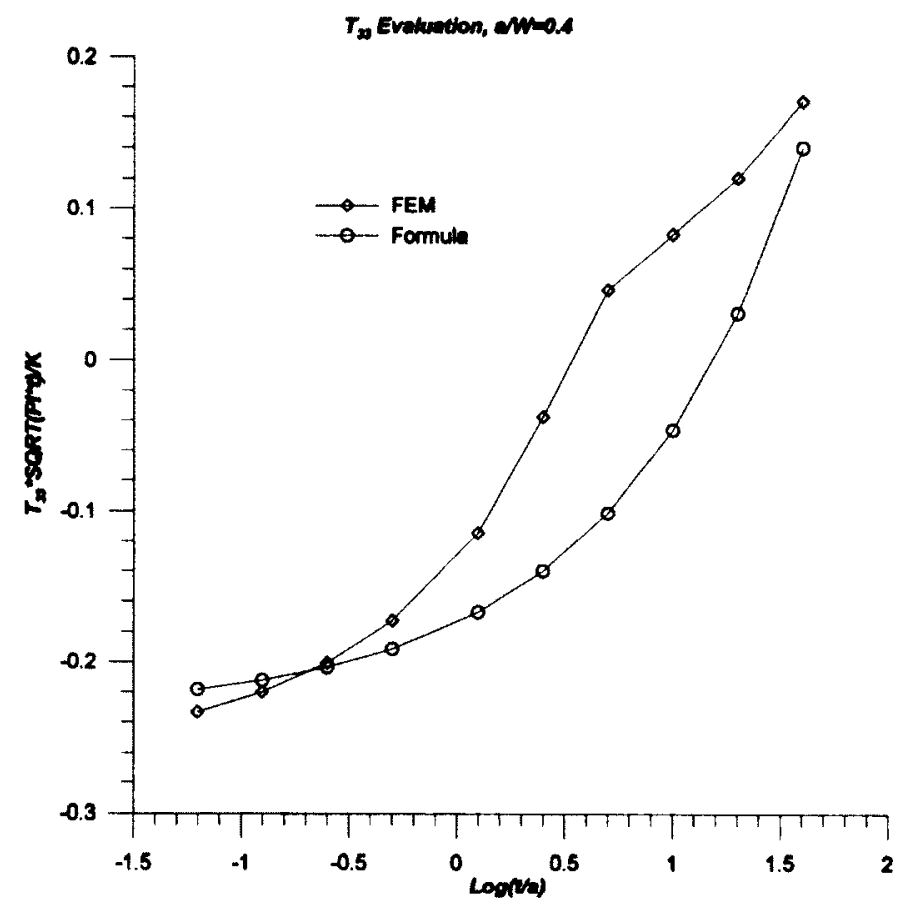

Figure 4.18 Comparison of approximation formula and FEM for $T_{33}, a / W=0.4$ 


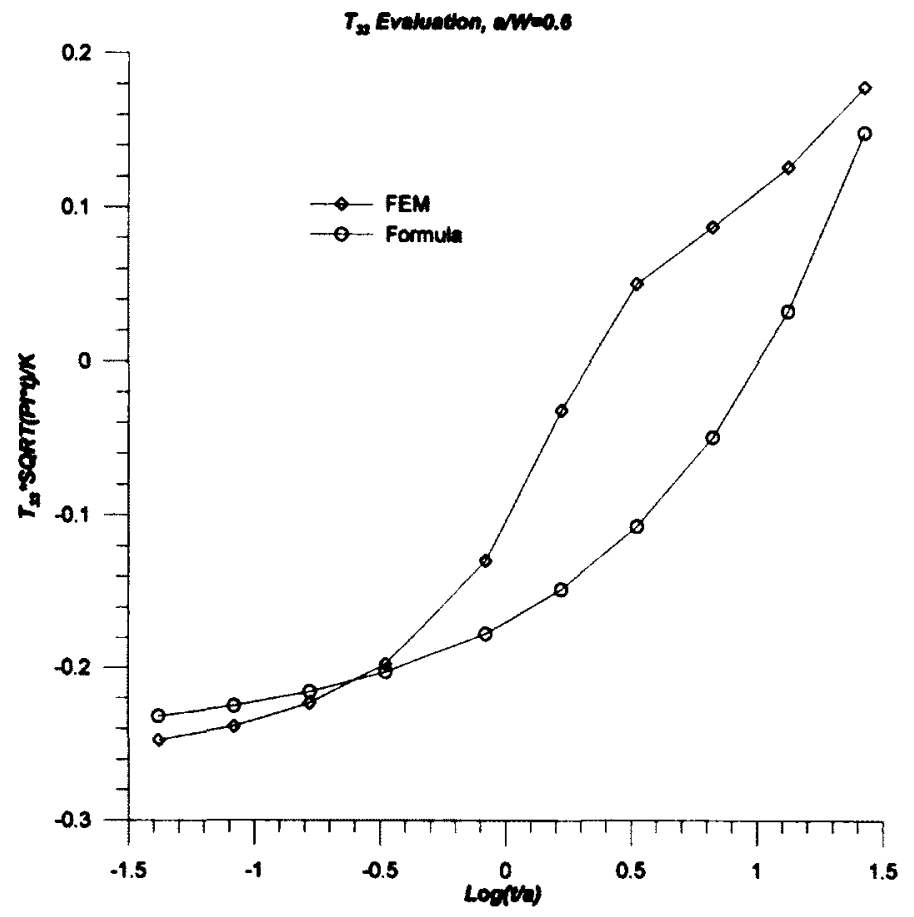

Figure 4.19 Comparison of approximation formula and FEM for $T_{33}, a / W=0.6$

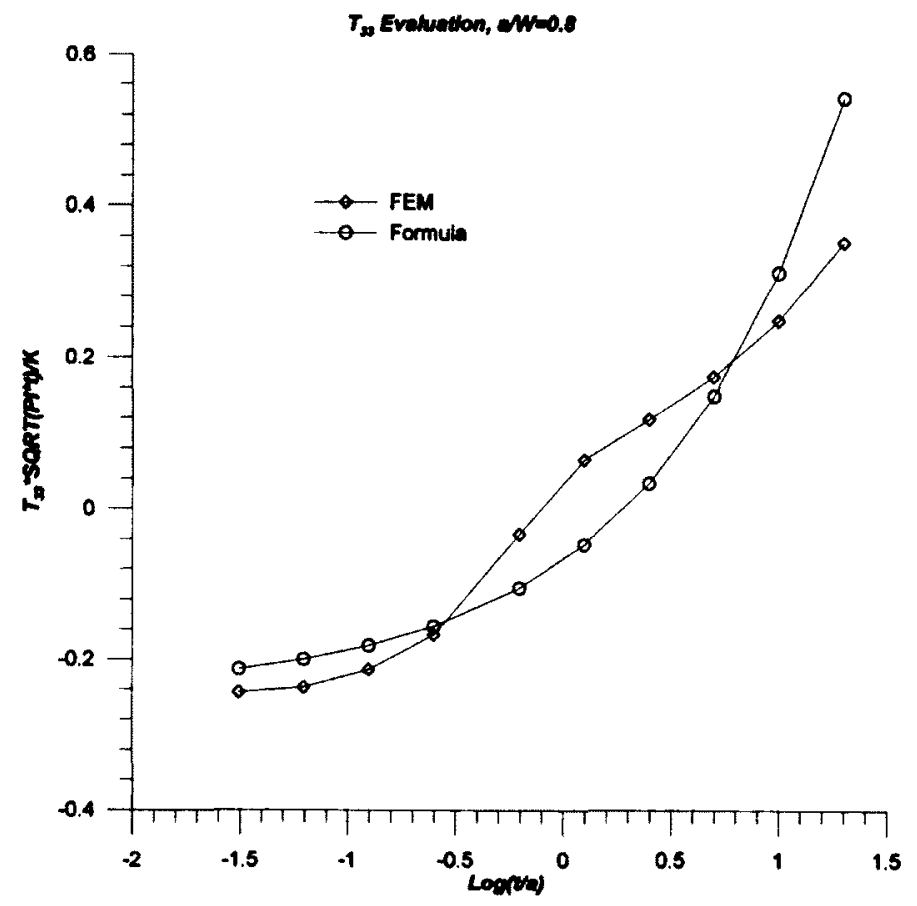

Figure 4.20 Comparison of approximation formula and FEM for $T_{33}, a / W=0.8$ 


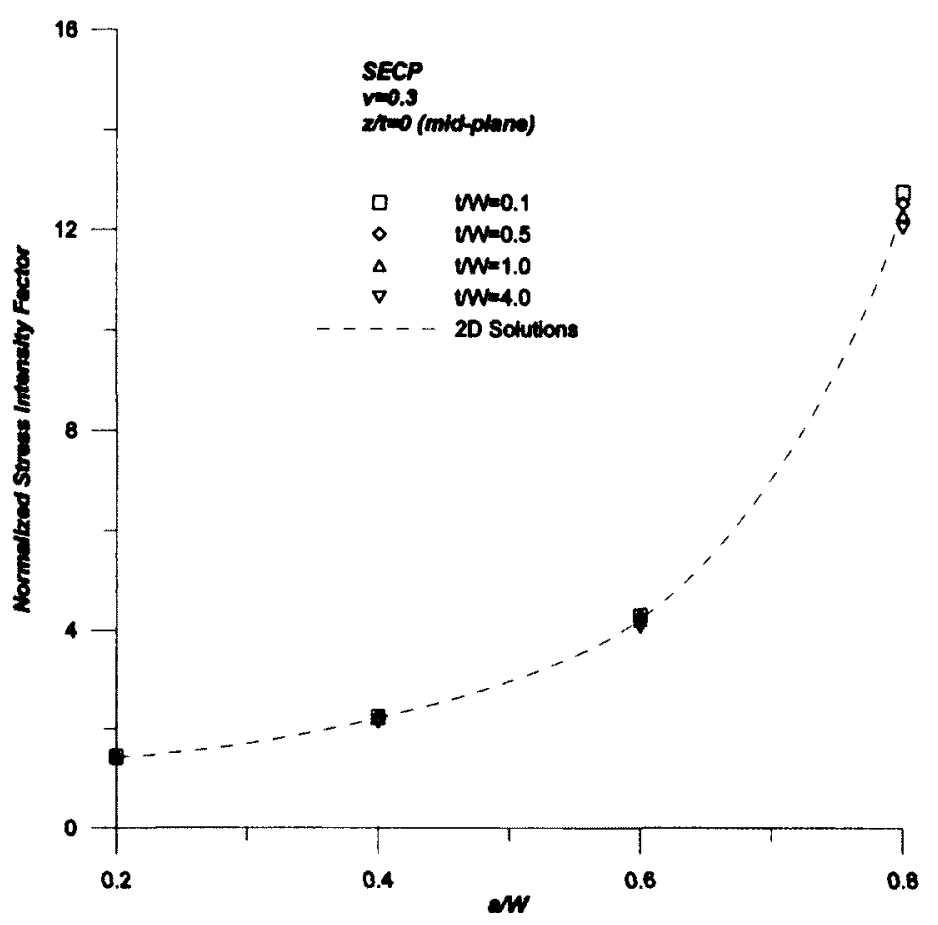

Figure 4.21 Mid-plane value of stress intensity factor as a function of crack depth in SECP

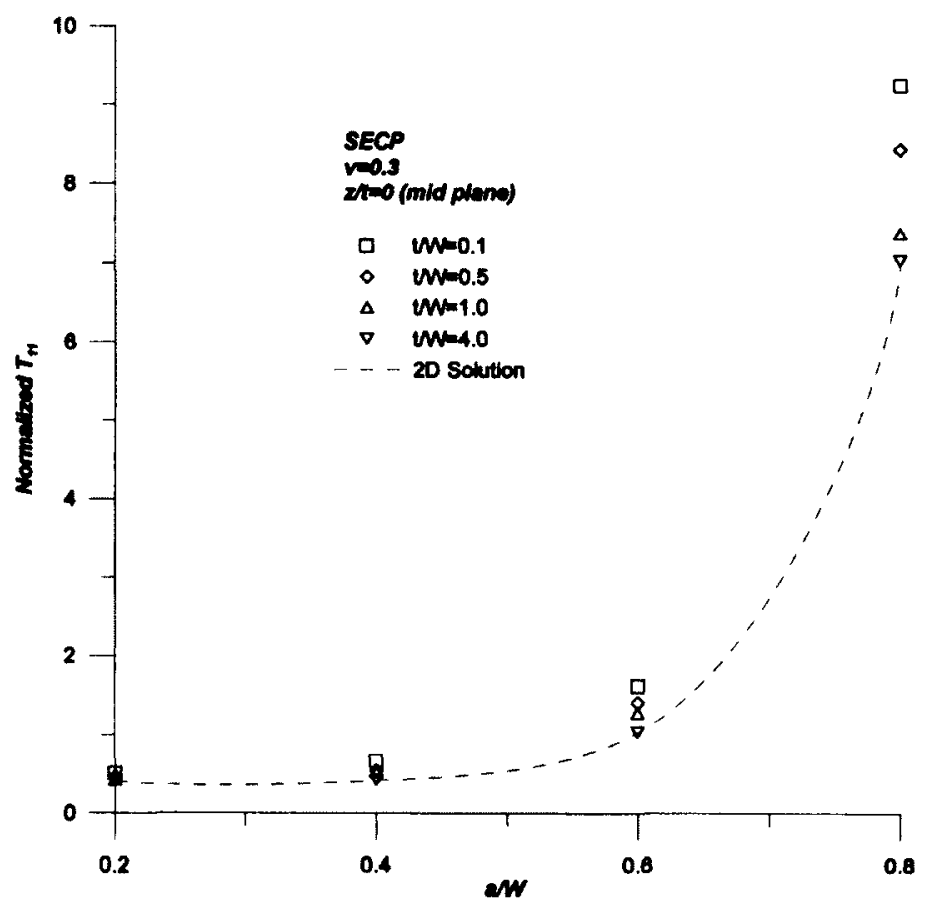

Figure 4.22 Mid-plane value of $T_{11}$ as a function of crack depth in SECP 


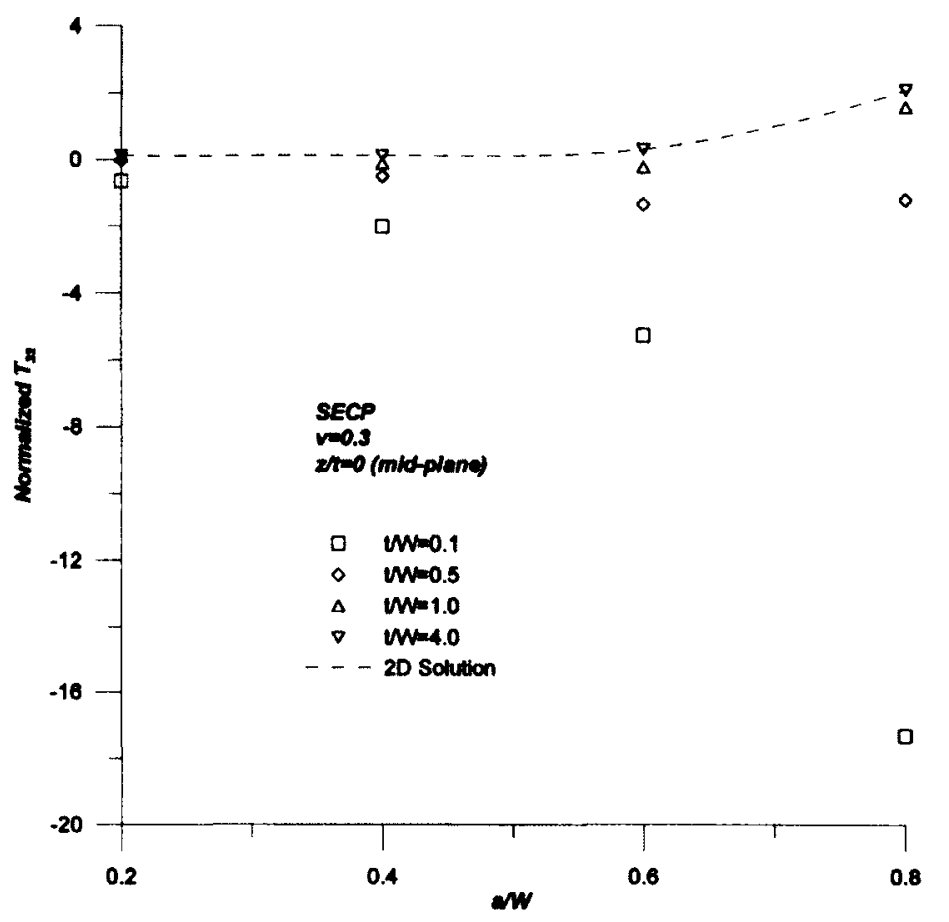

Figure 4.23 Mid-plane value of $T_{33}$ as a function of crack depth in SECP

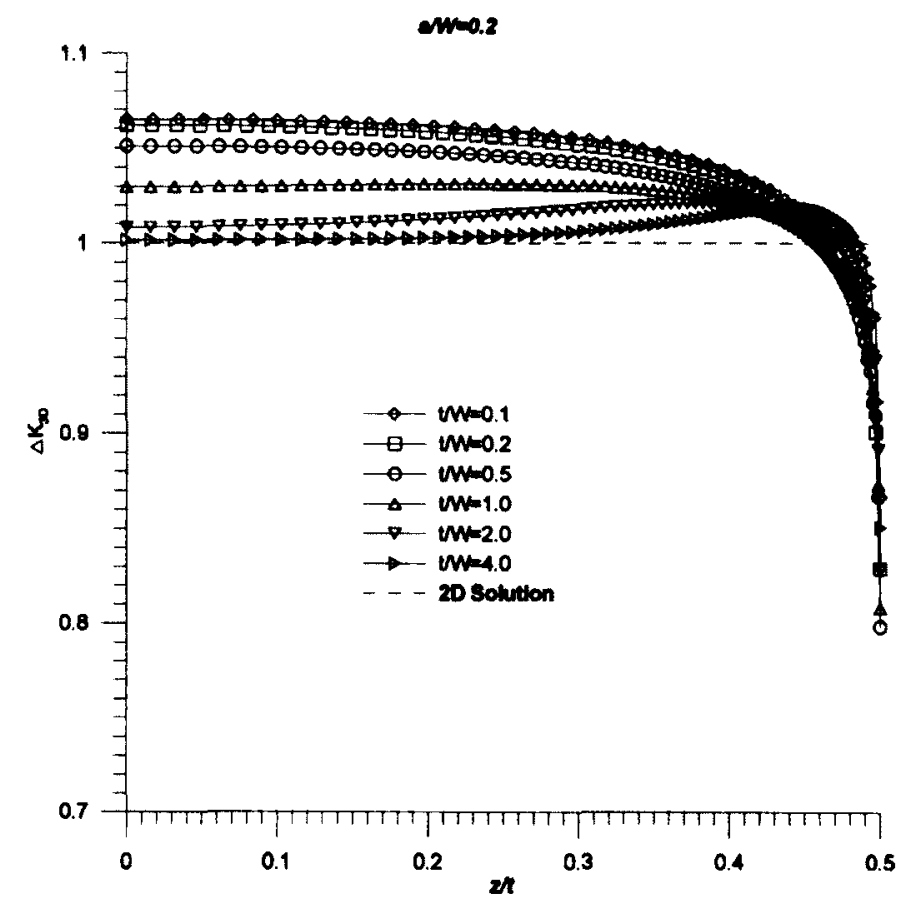

Figure 4.24 Variations of relative SIF along the crack tip for plates various thickness, uniform, $a / W=0.2$ 


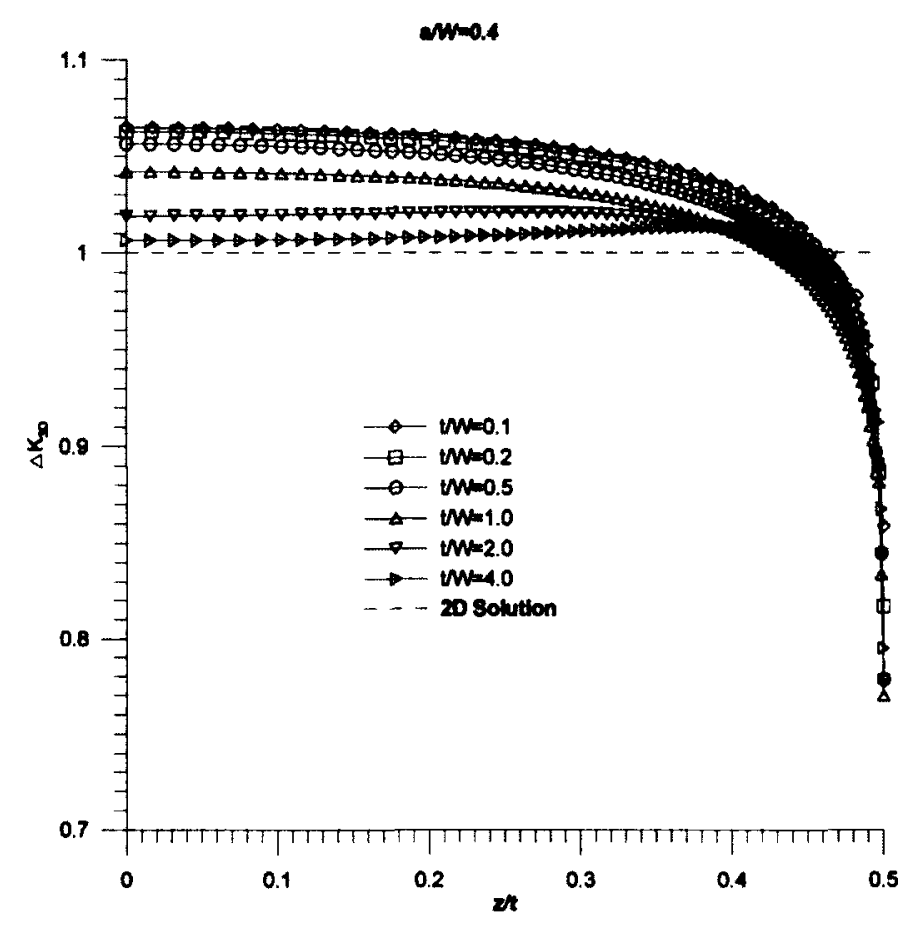

Figure 4.25 Variations of relative SIF along the crack tip for plates various thickness, uniform, $a / W=0.4$

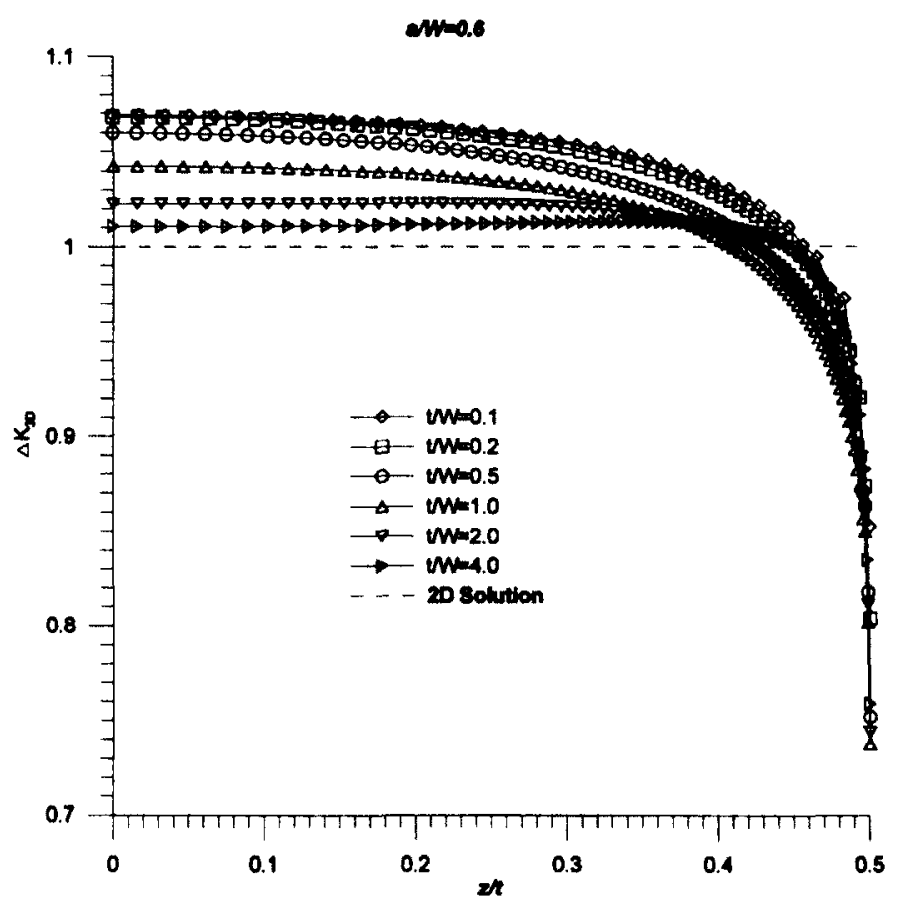

Figure 4.26 Variations of relative SIF along the crack tip for plates various thickness, uniform, $a / W=0.6$ 


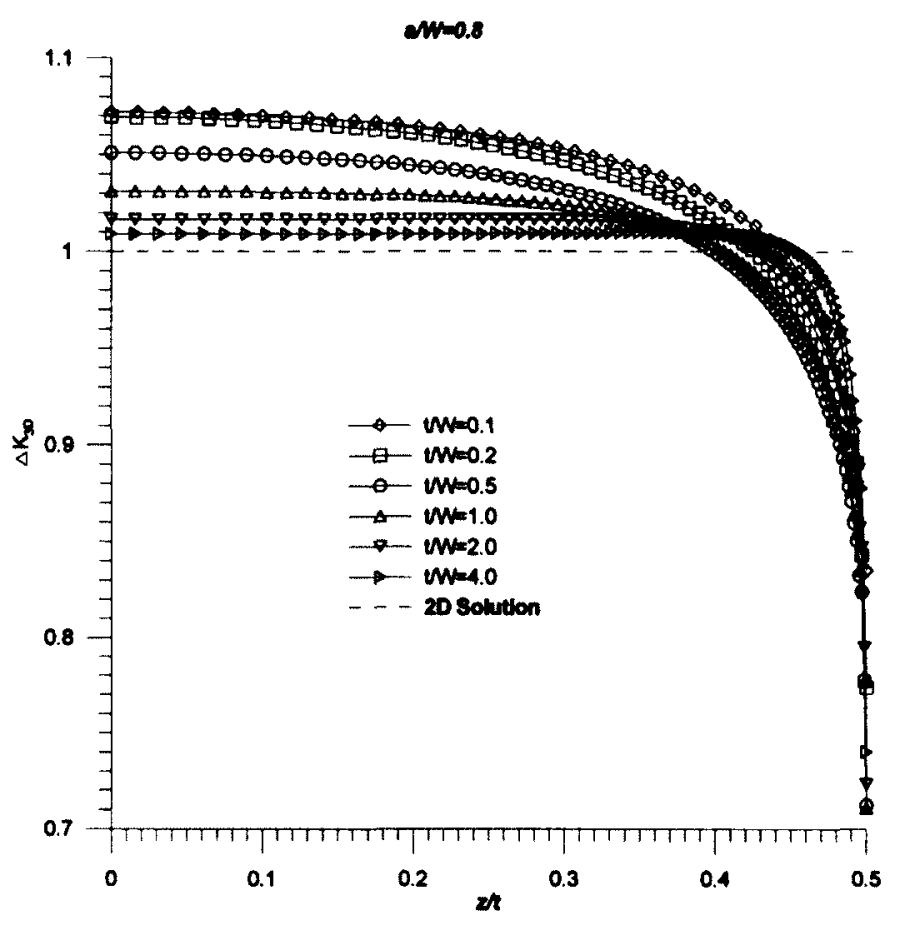

Figure 4.27 Variations of relative SIF along the crack tip for plates various thickness, uniform, $a / W=0.8$

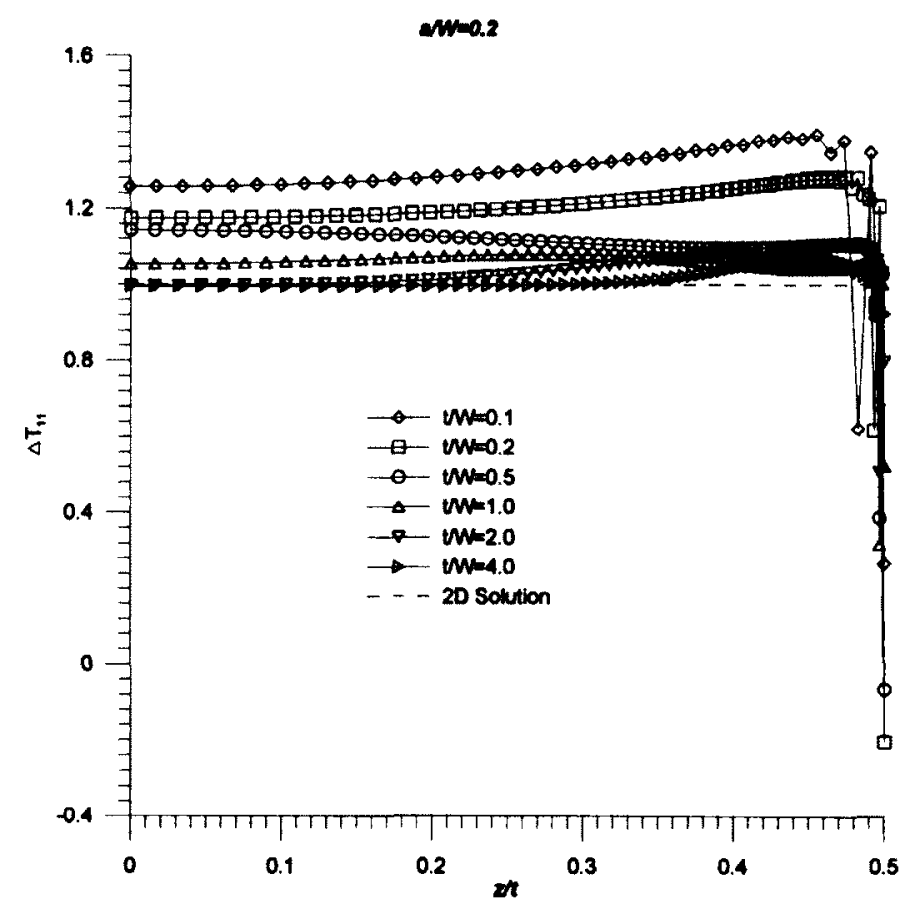

Figure 4.28 Variations of relative $T_{11}$ along the crack tip for plates various thickness, uniform, $a / W=0.2$ 


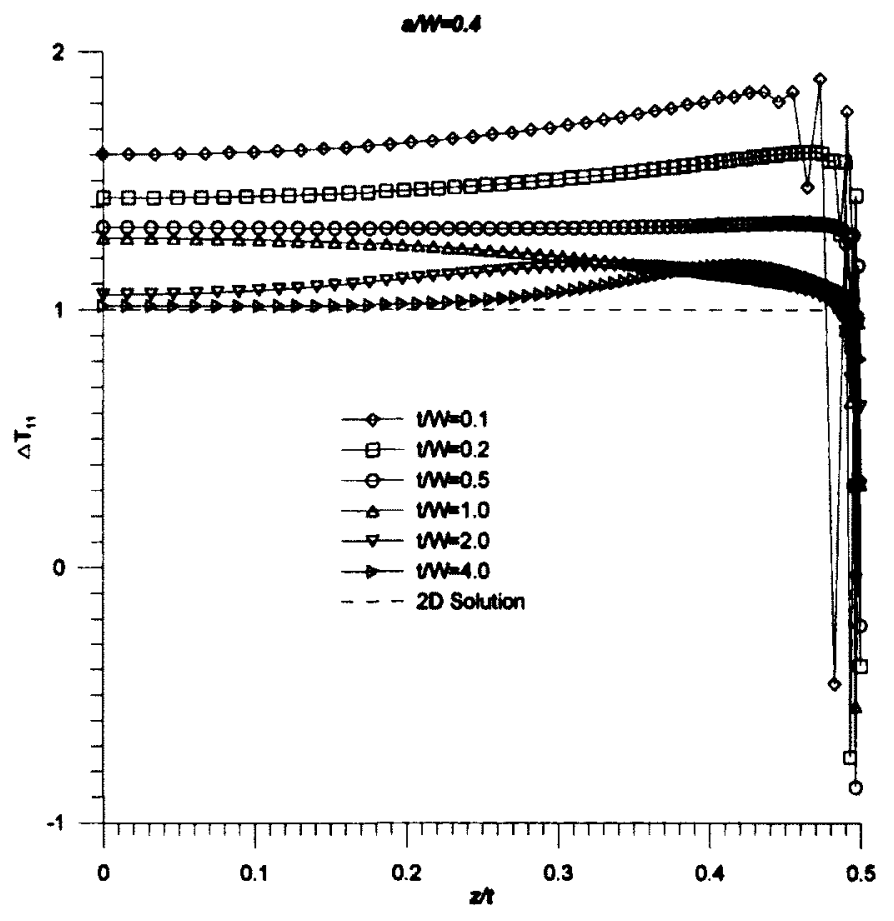

Figure 4.29 Variations of relative $T_{11}$ along the crack tip for plates various thickness, uniform, $a / W=0.4$

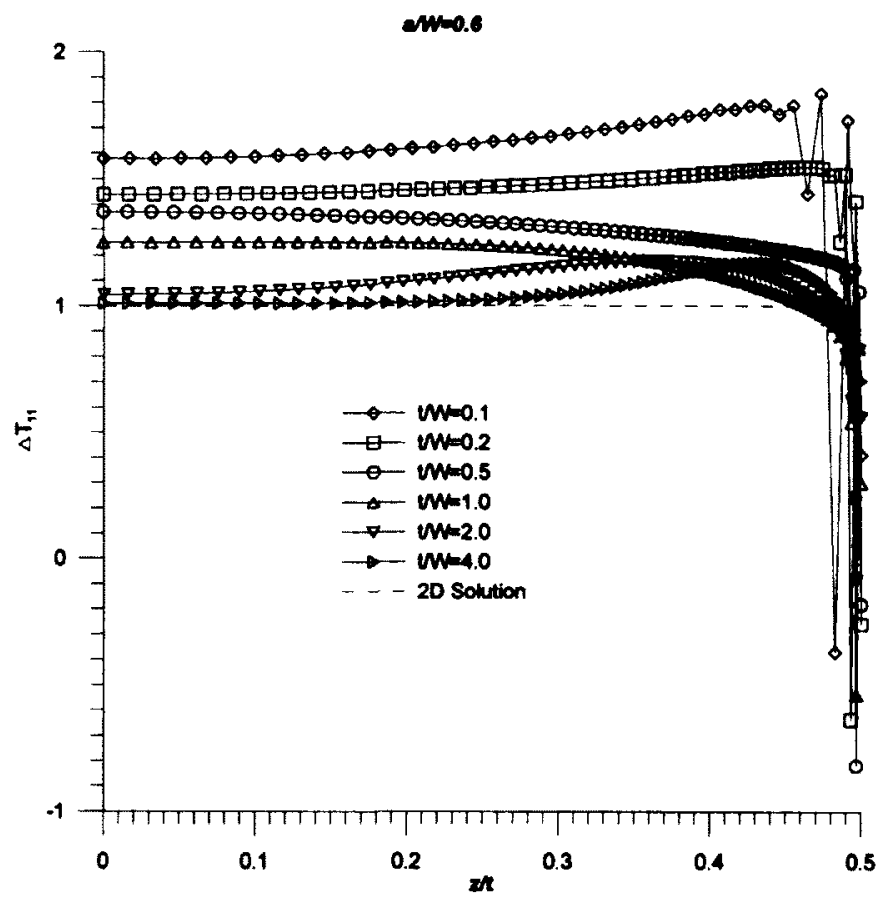

Figure 4.30 Variations of relative $T_{11}$ along the crack tip for plates various thickness, uniform, $a / W=0.6$ 


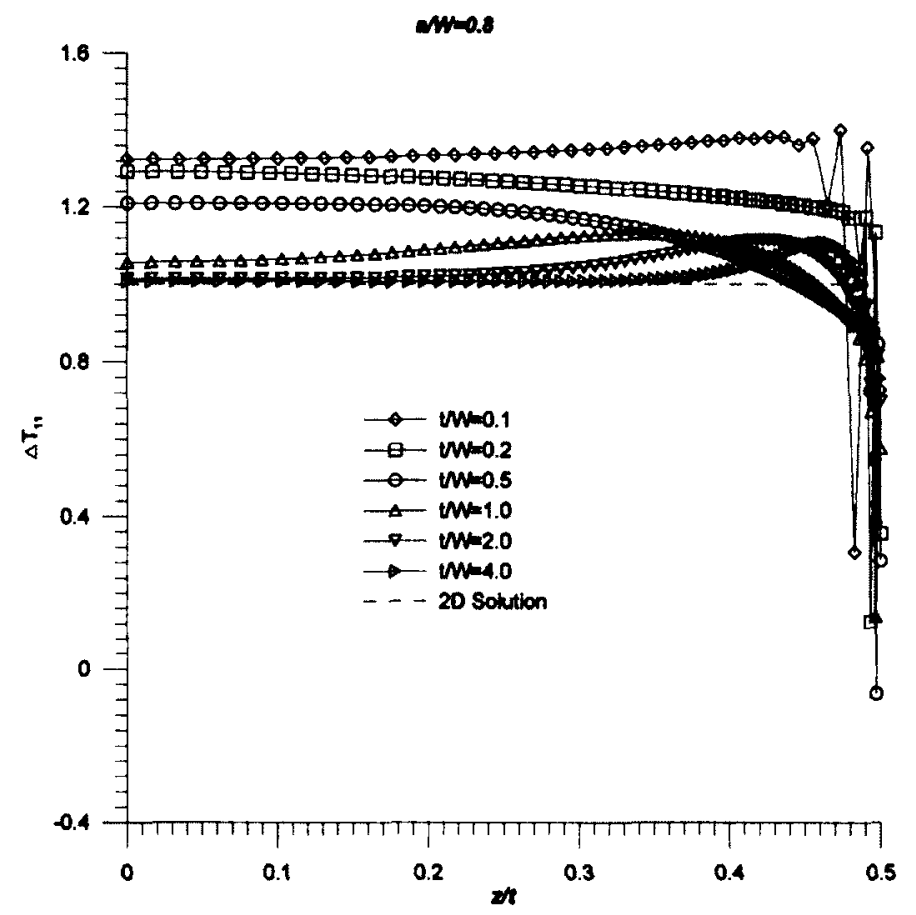

Figure 4.31 Variations of relative $T_{11}$ along the crack tip for plates various thickness, uniform, $a / W=0.8$

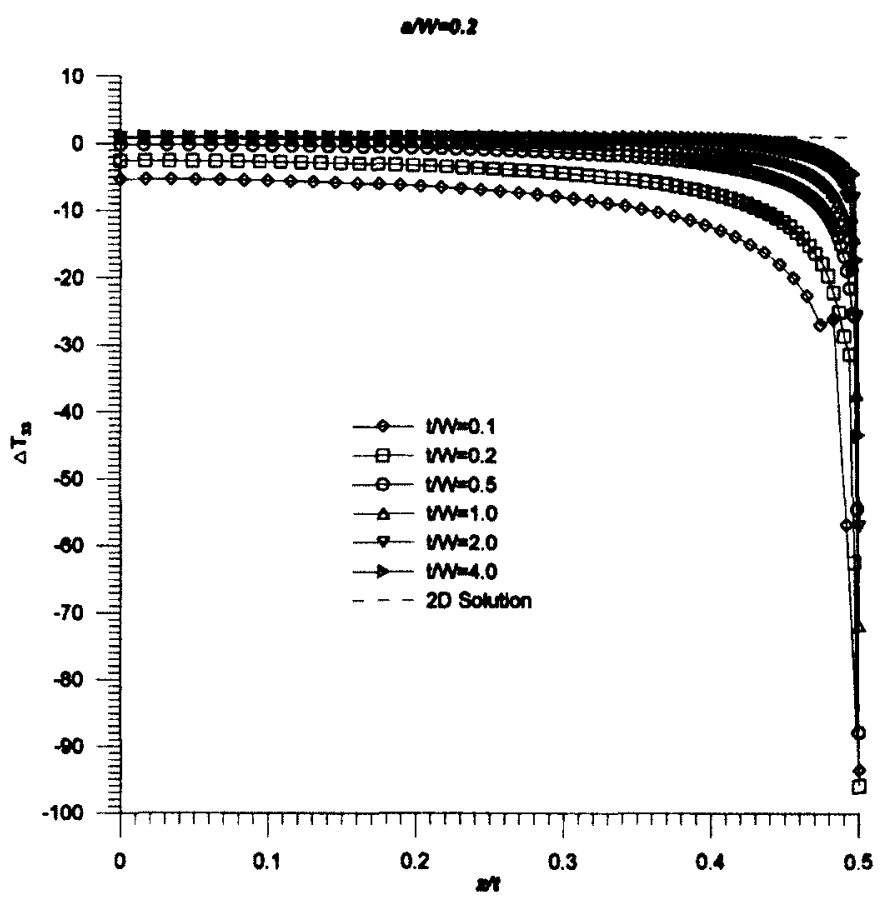

Figure 4.32 Variations of relative $T_{33}$ along the crack tip for plates various thickness, uniform, $a / W=0.2$ 


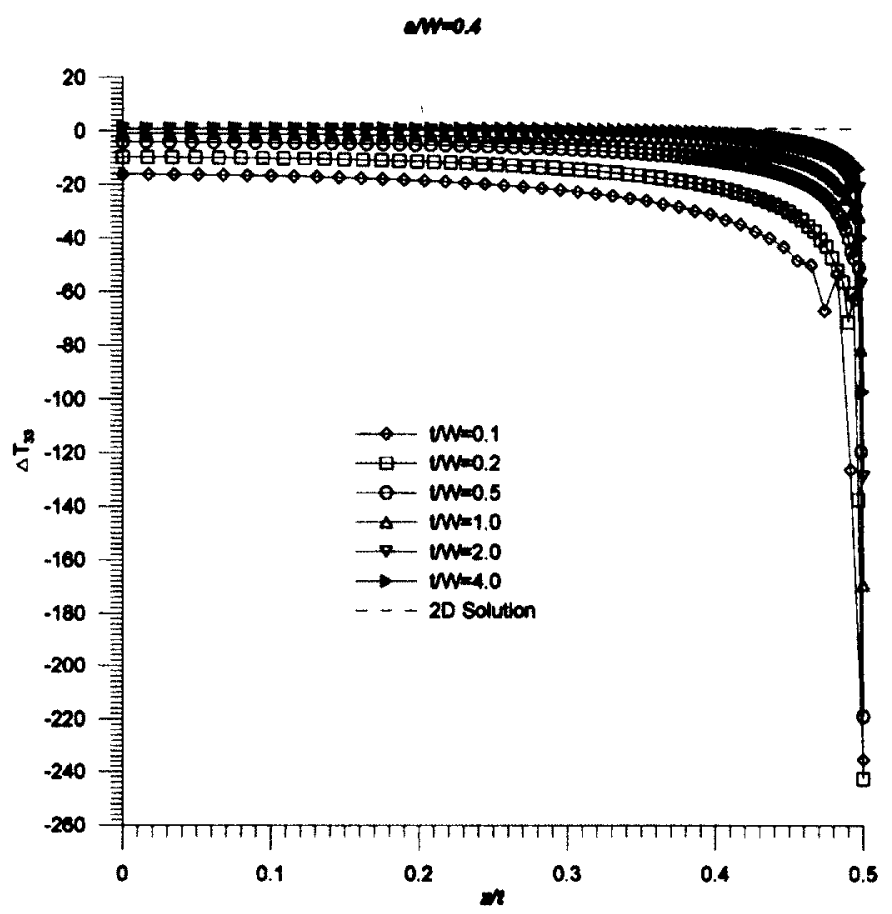

Figure 4.33 Variations of relative $T_{33}$ along the crack tip for plates various thickness, uniform, $a / W=0.4$

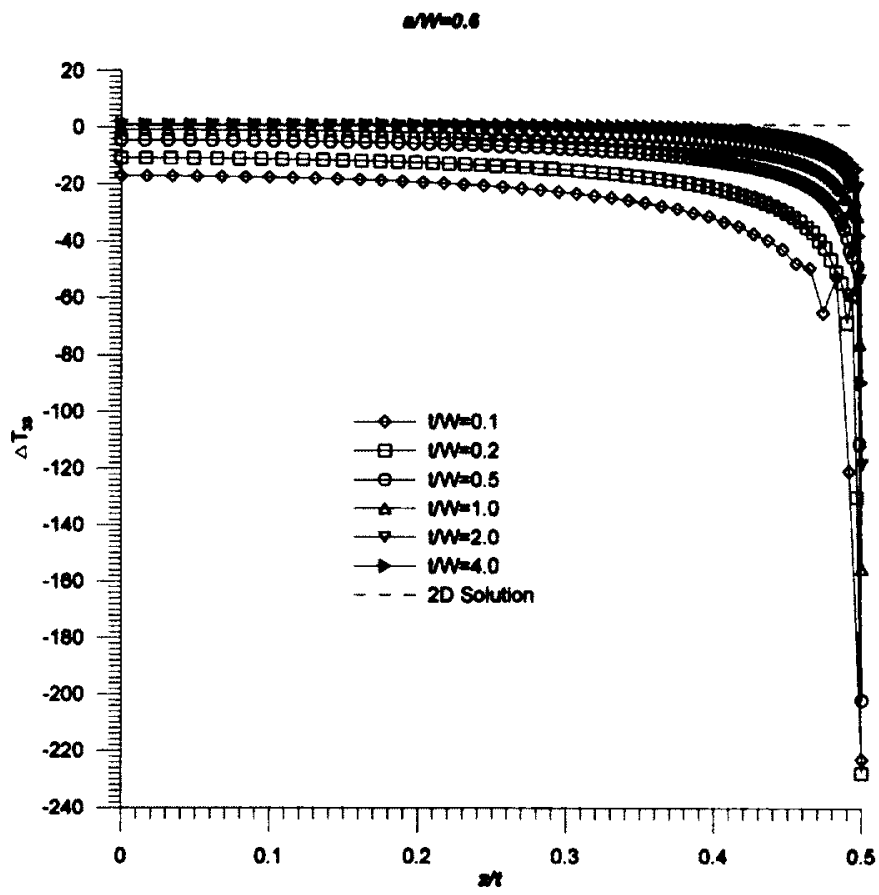

Figure 4.34 Variations of relative $T_{33}$ along the crack tip for plates various thickness, uniform, $a / W=0.6$ 


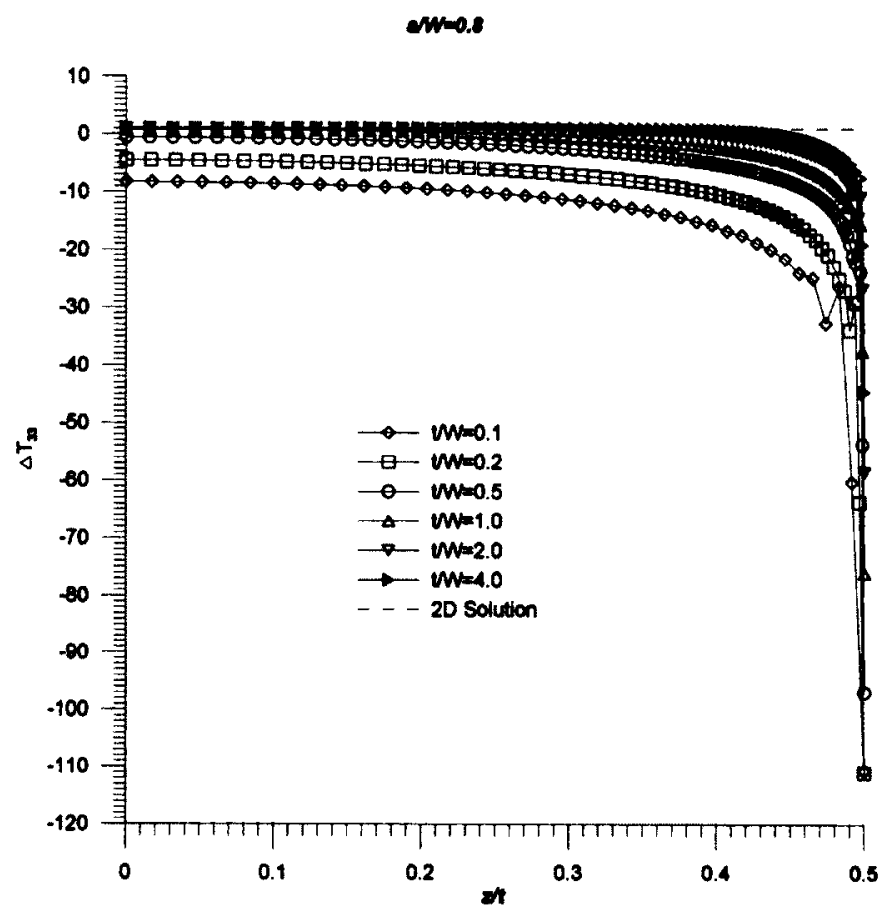

Figure 4.35 Variations of relative $T_{33}$ along the crack tip for plates various thickness, uniform, $a / W=0.8$ 


\section{CHAPTER 5}

\section{STRESS STATE DESCRIPTION BY $K_{1}, T_{11}$ AND $T_{33}$}

In this chapter, the stress state around the crack front in three-dimensional SECP specimens described by three parameters, stress intensity factor $K_{l}$, and $T$-stresses $\left(T_{11}\right.$ and $T_{33}$ ) from solutions presented in Chapter 3 are studied. The variation of the stress state described by Williams' expansion is compared with data obtained from finite element analyses by angle, radius and thickness, respectively.

\subsection{Stress State Description by $K_{1}, T_{11}$ and $T_{33}$}

The plane strain and plane stress state assumptions are widely used for two-dimensional problems for the development of stress state but cannot directly generalized to three-dimensional crack problem. However, it is accepted that using the description of the singular stress fields in the vicinity of the three-dimensional crack front according to the Williams series expansion (Williams, 1957). The stress state description using $K_{l}, T_{11}$ and $T_{33}$ is presented in the following. The stress state is studied at points located at a very small (but finite) radial distance to the crack front. The small circle has a radius $r$ and the polar angle $\theta$ is varied between $0^{\circ}$ and $180^{\circ}$. The coordinate system used at the crack front is shown in Figure 5.1.

From Williams' expansion, where higher order terms are neglected since the circular path is very close to the crack front, and the following relationships are obtained:

$$
\frac{\sigma_{i i}-T_{i i}}{K_{l} / \sqrt{2 \pi r}}=f_{i i}(\theta) \quad i=1,2,3
$$


With

$$
\begin{aligned}
& f_{11}(\theta)=\cos \frac{\theta}{2}\left(1-\sin \frac{\theta}{2} \sin \frac{3 \theta}{2}\right) \\
& f_{22}(\theta)=\cos \frac{\theta}{2}\left(1+\sin \frac{\theta}{2} \sin \frac{3 \theta}{2}\right) \\
& f_{33}(\theta)=v\left(f_{11}(\theta)+f_{22}(\theta)\right)=2 v \cos \frac{\theta}{2} \\
& \text { and } T_{22}=0
\end{aligned}
$$

In the following sections, these equations are used to predict the stress state using $K_{I}, T_{11}$ and $T_{33}$, and comparisons are made with finite element calculation results for the crack surface subjected to the uniform loading.

\subsubsection{Stress State Varied by Angle}

The results of finite element analyses for $K_{I}, T_{11}, T_{33}$, and $\sigma_{i i}(i=1,2,3)$ as described in Chapter 3 were substituted in Eq. (5.1), and compared with Eq. (5.2). The comparisons of $a / W=0.8$ varied by $t / W(t / W=0.1,0.2,0.5,1.0,2.0$, or 4.0$)$ at location $z / t=0$ (mid-plane) are shown in Figure 5.2-5.7. The angle $\theta$ is increased by $7.5^{\circ}$ from $0^{\circ}$ to $180^{\circ}$. The ratio of the radius from the crack front to the location chosen for stress state analysis between crack length, $r / a$, is fixed to $4.15 \mathrm{E}-3$. The results for the difference between the Eq. (5.2) and the finite element results are listed in Table 5.1-5.6. In general, the agreement between finite element results and formulae evaluation is very good at the analyzed angle for the different relative thickness, except the percentage of few values at particular angles are over $10 \%$.

Next, the model of the relative crack depth $a / W=0.8$ is used to analyze the percentage difference of $f_{i i}(\theta)$ s between the finite element results and formulae results varied by angle, and the results are shown in Figure 5.8-5.10. The relative thickness of 
the plate is $t / W=0.1,0.2,0.5,1.0,2.0$ and 4.0 , respectively. Three relative radiuses are chosen $(r / a=1.26559 \mathrm{E}-4,7.56476 \mathrm{E}-5$, or $3.61595 \mathrm{E}-5)$. The percentage difference of $f_{11}(\theta)$ varied by angles at three radiuses location is shown in Figure 5.8. The angle $\theta$ is increased by $15^{\circ}$ from $0^{\circ}$ to $165^{\circ}$. It shows that the peak of the difference is located on $75^{\circ}$ and decreasing to both sides. The percentage different of $f_{22}(\theta)$ varied by angles at three radiuses location is shown in Figure 5.9 (a) and (b). The angle $\theta$ is increased by $15^{\circ}$ from $0^{\circ}$ to $135^{\circ}$ in Figure 5.9 (a) and $0^{\circ}$ to $165^{\circ}$ in Figure 5.9 (b). It shows that the maximum difference appears around $165^{\circ}$, and also the difference is high around $0^{\circ}$. And the minimum difference appears around $90^{\circ}$. The Figure 5.10 shows the percentage difference of $f_{33}(\theta)$ varied by angles at three radiuses location. The angle $\theta$ is increased by $15^{\circ}$ from $0^{\circ}$ to $165^{\circ}$. The maximum difference is around $165^{\circ}$.

From Figure 5.8-5.10, it also notices that the percentage different of $f_{22}(\theta)$ is not varied significantly by thickness of the plate. And the percentage different of $f_{11}(\theta)$ and $f_{33}(\theta)$ is not varied significantly by the thickness of the plate when relative thickness $t / W>0.2$.

\subsubsection{Stress State Varied by Radius}

The stress state varied by radius for $a / W=0.6, t / W=0.5$ at four locations along thickness subjected by uniform stress field are compared, as shown in Figure 5.11-5.31. The angles are $0^{\circ}, 30^{\circ}, 60^{\circ}, 90^{\circ}, 120^{\circ}, 150^{\circ}$ and $180^{\circ}$, respectively. The four locations along thickness are at $z / t=0,0.11612,0.26369$ or 0.4182 , respectively. The difference between the finite element analysis results and Eq. (5.2) for $f_{11}(\theta)$ are shown in Figure 5.11-5.17. The finite element analysis gave good results for very small radius from crack front, and deviating from the results of Eq. (5.2) with the radius increasing. Stress State along the circular path close to the crack front varied by radius at four 
locations along thickness for $f_{22}(\theta)$ is shown in Figures 5.18-5.24. It is noticed that the difference between the finite element analysis and the Eq. (5.2) for $\mathrm{f}_{22}(\theta)$ are increasing with the relative radius ratio $r / a$ increasing. The finite element results of $f_{11}(\theta)$ and $f_{22}(\theta)$ are smaller than the results calculated from Eq. (5.2). Figures 5.25-5.31 show the $f_{33}(\theta)$ along the circular path close to the crack front varied by radius at four locations along thickness. The finite element analysis results are decreasing with the relative radius rate $r / a$ increasing for three locations along the thickness direction $(z / t=0,0.11612$, or $0.26369)$. But the values of the finite element analysis for position $z / t=0.4182$ are increasing and some of angles are large than the results of Eq. (5.2) finally $\left(\theta=150^{\circ}\right.$ or $\left.180^{\circ}\right)$.

The percentage difference of $f_{i i}(\theta) \mathrm{s}$ between finite element results and formulae results varied by radius of $a / W=0.6, t / W=0.5$, and $z / t=0$ (mid-plane) are listed in Table 5.7, and shown in Figure 5.32-5.37. The angles are $0^{\circ}, 30^{\circ}, 60^{\circ}, 90^{\circ}$, $120^{\circ}$, and $150^{\circ}$, respectively. It is noticed that the difference between the finite element and the Eq. (5.2) increases when the distance from the crack front increases for all three $f_{i i}(\theta)$ s. The accurate of the stress state description by the three parameters is changed by the angle for fixed radius.

\subsubsection{Stress State along Thickness}

The stress state varied by four locations along thickness for $a / W=0.6, t / W=0.1$, $0.2,0.5,1.0,2.0$ and 4.0, subjected by uniform stress field were compared, shown as in Fig. 5.38-5.55. And the results are shown in Table 5.8-5.13. The relative ratio of radius and crack length is fixed, $r / a=7.8 \mathrm{E}-3$. Due to the mesh difference, the four locations along thickness are $z / t=0,0.1311,0.2702$ or 0.3861 for $t / W=0.1, z / t=0,0.12277$, 0.26265 or 0.42441 for $t / W=0.2$, and $z / t=0,0.11612,0.26369$ or 0.4182 for $t / W=0.5$, 
1.0, 2.0 and 4.0. The stress state description by $K_{l}, T_{11}$ and $T_{33}$ with Williams formulae and finite element results are compared in the figures. For cases of $t / W=0.1$ and 0.2 , the results for first three locations along thickness $(z / t=0,0.1311$, and 0.2702 for $t / W=0.1$, and $z / t=0,0.12277$, and 0.26265 for $t / W=0.2$ ) are slight different. But the results for the locations near the free boundary $(z / t=0.3861$ for $t / W=0.1, z / t=0.42441$ for $t / W=0.2)$ are quite different than the others. There is very good agreement for near crack front stress state in mid-plane for $t / W \geq 0.5$. Except $f_{22}(\theta)$ for the case of $t / W=4.0$. The results of the other three locations along crack front are almost same but slightly different from the mid-plane evaluation for $t / W \geq 0.5$.

\subsection{Conclusion}

The stress state around the crack front for through-thickness single edge crack in plate under uniform loading was described by three parameters, stress intensity factor $K_{I}$, and $T$-stresses $\left(T_{11}\right.$ and $\left.T_{33}\right)$. Generally, when $r / a$ is small, the results of the finite element analyses and the Williams' expansion have very good fit for almost all cases. There is very good agreement for near crack front stress state described by $K_{l}, T_{11}$ and $T_{33}$ in mid-plane. The other locations along crack front are almost same but slight different from the Eq. (5.2). 
Table 5.1 Comparison of stress state at mid-plane between finite element results and formulae evaluation for $a / W=0.8, t / W=0.1$, Uniform Load, SECP

\begin{tabular}{|c|c|c|c|c|c|c|c|c|c|}
\hline $\begin{array}{c}\text { Degree } \\
(\end{array}$ & \multicolumn{3}{|c|}{$f_{11}(\theta)$} & \multicolumn{3}{|c|}{$f_{22}(\theta)$} & \multicolumn{3}{c|}{$f_{33}(\theta)$} \\
\cline { 2 - 10 } & Eq.(5.2) & FEM & $\%$ & Eq.(5.2) & FEM & $\%$ & Eq.(5.2) & FEM & $\%$ \\
\hline 0 & 1.0000 & 0.9751 & 2.49 & 1.0000 & 0.9554 & 4.46 & 0.6000 & 0.5738 & 4.37 \\
\hline 7.5 & 0.9851 & 0.9454 & 4.03 & 1.0106 & 0.9767 & 3.35 & 0.5987 & 0.5714 & 4.56 \\
\hline 15 & 0.9419 & 0.9150 & 2.85 & 1.0410 & 0.9989 & 4.04 & 0.5949 & 0.5690 & 4.34 \\
\hline 22.5 & 0.8745 & 0.8363 & 4.37 & 1.0871 & 1.0528 & 3.15 & 0.5885 & 0.5619 & 4.51 \\
\hline 30 & 0.7891 & 0.7571 & 4.06 & 1.1427 & 1.1073 & 3.09 & 0.5796 & 0.5548 & 4.27 \\
\hline 37.5 & 0.6938 & 0.6578 & 5.20 & 1.2000 & 1.1658 & 2.85 & 0.5682 & 0.5431 & 4.42 \\
\hline 45 & 0.5972 & 0.5586 & 6.47 & 1.2505 & 1.2243 & 2.10 & 0.5543 & 0.5313 & 4.15 \\
\hline 52.5 & 0.5078 & 0.4734 & 6.78 & 1.2859 & 1.2532 & 2.54 & 0.5381 & 0.5151 & 4.28 \\
\hline 60 & 0.4330 & 0.3888 & 10.21 & 1.2990 & 1.2817 & 1.33 & 0.5196 & 0.4989 & 3.98 \\
\hline 67.5 & 0.3784 & 0.3443 & 9.01 & 1.2845 & 1.2556 & 2.26 & 0.4989 & 0.4785 & 4.09 \\
\hline 75 & 0.3472 & 0.3006 & 13.40 & 1.2396 & 1.2286 & 0.88 & 0.4760 & 0.4581 & 3.77 \\
\hline 82.5 & 0.3397 & 0.3046 & 10.34 & 1.1640 & 1.1408 & 2.00 & 0.4511 & 0.4337 & 3.86 \\
\hline 90 & 0.3536 & 0.3092 & 12.53 & 1.0607 & 1.0522 & 0.80 & 0.4243 & 0.4094 & 3.51 \\
\hline 97.5 & 0.3839 & 0.3474 & 9.51 & 0.9348 & 0.9182 & 1.77 & 0.3956 & 0.3814 & 3.58 \\
\hline 105 & 0.4239 & 0.3860 & 8.95 & 0.7936 & 0.7839 & 1.22 & 0.3653 & 0.3535 & 3.21 \\
\hline 112.5 & 0.4655 & 0.4284 & 7.95 & 0.6457 & 0.6355 & 1.58 & 0.3333 & 0.3225 & 3.26 \\
\hline 120 & 0.5000 & 0.4707 & 5.86 & 0.5000 & 0.4872 & 2.56 & 0.3000 & 0.2914 & 2.86 \\
\hline 127.5 & 0.5197 & 0.4843 & 6.82 & 0.3649 & 0.3593 & 1.54 & 0.2654 & 0.2577 & 2.89 \\
\hline 135 & 0.5180 & 0.4972 & 4.02 & 0.2474 & 0.2320 & 6.22 & 0.2296 & 0.2240 & 2.43 \\
\hline 142.5 & 0.4905 & 0.4595 & 6.33 & 0.1523 & 0.1489 & 2.27 & 0.1929 & 0.1882 & 2.40 \\
\hline 150 & 0.4356 & 0.4209 & 3.36 & 0.0820 & 0.0666 & 18.78 & 0.1553 & 0.1524 & 1.84 \\
\hline 157.5 & 0.3542 & 0.3302 & 6.77 & 0.0360 & 0.0323 & 10.29 & 0.1171 & 0.1151 & 1.64 \\
\hline 165 & 0.2501 & 0.2388 & 4.53 & 0.0110 & -0.0013 & 112.02 & 0.0783 & 0.0778 & 0.61 \\
\hline 172.5 & 0.1294 & 0.1146 & 11.43 & 0.0014 & -0.0043 & 411.68 & 0.0392 & 0.0397 & 1.09 \\
\hline 180 & 0.0000 & -0.0086 & Inf. & 0.0000 & -0.0087 & Inf. & 0.0000 & 0.0014 & Inf. \\
\hline
\end{tabular}


Table5.2 Comparison of stress state at mid-plane between finite element results and formulae evaluation for $a / W=0.8, t / W=0.2$, Uniform Load, SECP

\begin{tabular}{|c|c|c|c|c|c|c|c|c|c|}
\hline $\begin{array}{c}\text { Degree } \\
(\mathcal{G}\end{array}$ & \multicolumn{3}{|c|}{$f_{11}(\theta)$} & \multicolumn{3}{|c|}{$f_{22}(\theta)$} & \multicolumn{3}{|c|}{$f_{33}(\theta)$} \\
\hline 0 & Eq.(5.2) & FEM & $\%$ & Eq.(5.2) & FEM & $\%$ & Eq.(5.2) & FEM & $\%$ \\
\hline 7.5 & 0.0000 & 0.9756 & 2.44 & 1.0000 & 0.9568 & 4.32 & 0.6000 & 0.5787 & 3.56 \\
\hline 15 & 0.9419 & 0.9460 & 3.97 & 1.0106 & 0.9781 & 3.21 & 0.5987 & 0.5762 & 3.76 \\
\hline 22.5 & 0.8745 & 0.8369 & 4.29 & 1.0871 & 1.0542 & 3.03 & 0.5885 & 0.5664 & 3.76 \\
\hline 30 & 0.7891 & 0.7578 & 3.97 & 1.1427 & 1.1086 & 2.98 & 0.5796 & 0.5590 & 3.54 \\
\hline 37.5 & 0.6938 & 0.6586 & 5.08 & 1.2000 & 1.1670 & 2.75 & 0.5682 & 0.5469 & 3.74 \\
\hline 45 & 0.5972 & 0.5595 & 6.32 & 1.2505 & 1.2254 & 2.01 & 0.5543 & 0.5348 & 3.53 \\
\hline 52.5 & 0.5078 & 0.4744 & 6.58 & 1.2859 & 1.2543 & 2.46 & 0.5381 & 0.5181 & 3.73 \\
\hline 60 & 0.4330 & 0.3899 & 9.95 & 1.2990 & 1.2827 & 1.26 & 0.5196 & 0.5014 & 3.51 \\
\hline 67.5 & 0.3784 & 0.3456 & 8.68 & 1.2845 & 1.2564 & 2.19 & 0.4989 & 0.4803 & 3.72 \\
\hline 75 & 0.3472 & 0.3020 & 13.00 & 1.2396 & 1.2293 & 0.82 & 0.4760 & 0.4593 & 3.50 \\
\hline 82.5 & 0.3397 & 0.3061 & 9.89 & 1.1640 & 1.1414 & 1.94 & 0.4511 & 0.4343 & 3.72 \\
\hline 90 & 0.3536 & 0.3109 & 12.07 & 1.0607 & 1.0527 & 0.75 & 0.4243 & 0.4093 & 3.52 \\
\hline 97.5 & 0.3839 & 0.3491 & 9.07 & 0.9348 & 0.9187 & 1.72 & 0.3956 & 0.3808 & 3.75 \\
\hline 105 & 0.4239 & 0.3878 & 8.53 & 0.7936 & 0.7843 & 1.17 & 0.3653 & 0.3522 & 3.57 \\
\hline 112.5 & 0.4655 & 0.4303 & 7.55 & 0.6457 & 0.6357 & 1.54 & 0.3333 & 0.3206 & 3.84 \\
\hline 120 & 0.5000 & 0.4726 & 5.47 & 0.5000 & 0.4874 & 2.52 & 0.3000 & 0.2889 & 3.70 \\
\hline 127.5 & 0.5197 & 0.4862 & 6.45 & 0.3649 & 0.3594 & 1.51 & 0.2654 & 0.2547 & 4.03 \\
\hline 135 & 0.5180 & 0.4991 & 3.65 & 0.2474 & 0.2321 & 6.19 & 0.2296 & 0.2205 & 3.98 \\
\hline 142.5 & 0.4905 & 0.4613 & 5.96 & 0.1523 & 0.1489 & 2.24 & 0.1929 & 0.1843 & 4.45 \\
\hline 150 & 0.4356 & 0.4227 & 2.96 & 0.0820 & 0.0666 & 18.77 & 0.1553 & 0.1481 & 4.64 \\
\hline 157.5 & 0.3542 & 0.3318 & 6.31 & 0.0360 & 0.0323 & 10.30 & 0.1171 & 0.1106 & 5.56 \\
\hline 165 & 0.2501 & 0.2402 & 3.93 & 0.0110 & -0.0013 & 112.15 & 0.0783 & 0.0730 & 6.76 \\
\hline 172.5 & 0.1294 & 0.1159 & 10.43 & 0.0014 & -0.0044 & 412.75 & 0.0392 & 0.0348 & 11.34 \\
\hline 180 & 0.0000 & -0.0075 & Inf. & 0.0000 & -0.0087 & Inf. & 0.0000 & -0.0036 & Inf. \\
\hline & & & & & & & & & \\
\hline
\end{tabular}


Table 5.3 Comparison of stress state at mid-plane between finite element results and formulae evaluation for $a / W=0.8, t / W=0.5$, Uniform Load, SECP

\begin{tabular}{|c|c|c|c|c|c|c|c|c|c|}
\hline $\begin{array}{c}\text { Degree } \\
()\end{array}$ & \multicolumn{3}{|c|}{$f_{11}(\theta)$} & \multicolumn{3}{|c|}{$f_{22}(\theta)$} & \multicolumn{3}{|c|}{$f_{33}(\theta)$} \\
\hline 0 & Eq.(5.2) & FEM & $\%$ & Eq.(5.2) & FEM & $\%$ & Eq.(5.2) & FEM & $\%$ \\
\hline 7.5 & 0.9000 & 0.9831 & 1.69 & 1.0000 & 0.9647 & 3.53 & 0.6000 & 0.5847 & 2.56 \\
\hline 15 & 0.9419 & 0.9535 & 3.21 & 1.0106 & 0.9860 & 2.44 & 0.5987 & 0.5822 & 2.76 \\
\hline 22.5 & 0.8745 & 0.8444 & 2.00 & 1.0410 & 1.0081 & 3.16 & 0.5949 & 0.5797 & 2.55 \\
\hline 30 & 0.7891 & 0.7652 & 3.03 & 1.1427 & 1.1165 & 2.29 & 0.5796 & 0.5648 & 2.54 \\
\hline 37.5 & 0.6938 & 0.6661 & 4.00 & 1.2000 & 1.1749 & 2.09 & 0.5682 & 0.5526 & 2.75 \\
\hline 45 & 0.5972 & 0.5670 & 5.07 & 1.2505 & 1.2333 & 1.38 & 0.5543 & 0.5403 & 2.53 \\
\hline 52.5 & 0.5078 & 0.4819 & 5.10 & 1.2859 & 1.2621 & 1.85 & 0.5381 & 0.5234 & 2.73 \\
\hline 60 & 0.4330 & 0.3975 & 8.21 & 1.2990 & 1.2905 & 0.66 & 0.5196 & 0.5066 & 2.51 \\
\hline 67.5 & 0.3784 & 0.3531 & 6.68 & 1.2845 & 1.2642 & 1.58 & 0.4989 & 0.4853 & 2.71 \\
\hline 75 & 0.3472 & 0.3097 & 10.80 & 1.2396 & 1.2371 & 0.20 & 0.4760 & 0.4641 & 2.49 \\
\hline 82.5 & 0.3397 & 0.3137 & 7.63 & 1.1640 & 1.1491 & 1.28 & 0.4511 & 0.4389 & 2.70 \\
\hline 90 & 0.3536 & 0.3186 & 9.88 & 1.0607 & 1.0604 & 0.03 & 0.4243 & 0.4137 & 2.48 \\
\hline 97.5 & 0.3839 & 0.3569 & 7.03 & 0.9348 & 0.9262 & 0.91 & 0.3956 & 0.3849 & 2.70 \\
\hline 105 & 0.4239 & 0.3957 & 6.67 & 0.7936 & 0.7918 & 0.23 & 0.3653 & 0.3562 & 2.49 \\
\hline 112.5 & 0.4655 & 0.4382 & 5.86 & 0.6457 & 0.6432 & 0.39 & 0.3333 & 0.3243 & 2.71 \\
\hline 120 & 0.5000 & 0.4806 & 3.89 & 0.5000 & 0.4947 & 1.05 & 0.3000 & 0.2925 & 2.51 \\
\hline 127.5 & 0.5197 & 0.4941 & 4.92 & 0.3649 & 0.3667 & 0.49 & 0.2654 & 0.2581 & 2.75 \\
\hline 135 & 0.5180 & 0.5070 & 2.12 & 0.2474 & 0.2393 & 3.26 & 0.2296 & 0.2237 & 2.57 \\
\hline 142.5 & 0.4905 & 0.4692 & 4.34 & 0.1523 & 0.1561 & 2.49 & 0.1929 & 0.1874 & 2.84 \\
\hline 150 & 0.4356 & 0.4306 & 1.14 & 0.0820 & 0.0738 & 10.03 & 0.1553 & 0.1511 & 2.71 \\
\hline 157.5 & 0.3542 & 0.3397 & 4.09 & 0.0360 & 0.0394 & 9.60 & 0.1171 & 0.1135 & 3.06 \\
\hline 165 & 0.2501 & 0.2480 & 0.82 & 0.0110 & 0.0058 & 46.98 & 0.0783 & 0.0759 & 3.13 \\
\hline 172.5 & 0.1294 & 0.1236 & 4.48 & 0.0014 & 0.0028 & 99.89 & 0.0392 & 0.0376 & 4.14 \\
\hline 180 & 0.0000 & 0.0001 & Inf. & 0.0000 & -0.0016 & Inf. & 0.0000 & -0.0008 & Inf. \\
\hline & & & & & & & & & \\
\hline
\end{tabular}


Table 5.4 Comparison of stress state at mid-plane between finite element results and formulae evaluation for $a / W=0.8, t / W=1.0$, Uniform Load, SECP

\begin{tabular}{|c|c|c|c|c|c|c|c|c|c|}
\hline $\begin{array}{c}\text { Degree } \\
()\end{array}$ & \multicolumn{3}{|c|}{$f_{11}(\theta)$} & \multicolumn{3}{|c|}{$f_{22}(\theta)$} & \multicolumn{3}{c|}{$f_{33}(\theta)$} \\
\hline \multirow{nyyyyyyyyy}{*}{ Eq.(5.2) } & FEM & $\%$ & Eq.(5.2) & FEM & $\%$ & Eq.(5.2) & FEM & $\%$ \\
\hline 7.5 & 1.0000 & 0.9827 & 1.73 & 1.0000 & 0.9643 & 3.57 & 0.6000 & 0.5842 & 2.63 \\
\hline 15 & 0.9851 & 0.9531 & 3.25 & 1.0106 & 0.9856 & 2.47 & 0.5987 & 0.5817 & 2.84 \\
\hline 22.5 & 0.8745 & 0.9227 & 2.04 & 1.0410 & 1.0077 & 3.19 & 0.5949 & 0.5793 & 2.62 \\
\hline 30 & 0.7891 & 0.7649 & 3.48 & 1.0871 & 1.0617 & 2.34 & 0.5885 & 0.5718 & 2.83 \\
\hline 37.5 & 0.6938 & 0.6657 & 4.05 & 1.1427 & 1.1161 & 2.32 & 0.5796 & 0.5644 & 2.61 \\
\hline 45 & 0.5972 & 0.5666 & 5.13 & 1.2505 & 1.2329 & 1.41 & 0.5543 & 0.5400 & 2.59 \\
\hline 52.5 & 0.5078 & 0.4816 & 5.16 & 1.2859 & 1.2618 & 1.88 & 0.5381 & 0.5231 & 2.79 \\
\hline 60 & 0.4330 & 0.3971 & 8.28 & 1.2990 & 1.2902 & 0.68 & 0.5196 & 0.5063 & 2.57 \\
\hline 67.5 & 0.3784 & 0.3528 & 6.76 & 1.2845 & 1.2639 & 1.61 & 0.4989 & 0.4851 & 2.77 \\
\hline 75 & 0.3472 & 0.3094 & 10.89 & 1.2396 & 1.2368 & 0.22 & 0.4760 & 0.4639 & 2.55 \\
\hline 82.5 & 0.3397 & 0.3134 & 7.72 & 1.1640 & 1.1489 & 1.30 & 0.4511 & 0.4387 & 2.75 \\
\hline 90 & 0.3536 & 0.3183 & 9.97 & 1.0607 & 1.0602 & 0.05 & 0.4243 & 0.4136 & 2.53 \\
\hline 97.5 & 0.3839 & 0.3566 & 7.11 & 0.9348 & 0.9261 & 0.93 & 0.3956 & 0.3848 & 2.73 \\
\hline 105 & 0.4239 & 0.3954 & 6.74 & 0.7936 & 0.7916 & 0.25 & 0.3653 & 0.3561 & 2.51 \\
\hline 112.5 & 0.4655 & 0.4379 & 5.91 & 0.6457 & 0.6431 & 0.41 & 0.3333 & 0.3243 & 2.73 \\
\hline 120 & 0.5000 & 0.4803 & 3.94 & 0.5000 & 0.4947 & 1.07 & 0.3000 & 0.2924 & 2.52 \\
\hline 127.5 & 0.5197 & 0.4939 & 4.96 & 0.3649 & 0.3666 & 0.48 & 0.2654 & 0.2581 & 2.74 \\
\hline 135 & 0.5180 & 0.5068 & 2.16 & 0.2474 & 0.2393 & 3.28 & 0.2296 & 0.2238 & 2.55 \\
\hline 142.5 & 0.4905 & 0.4691 & 4.38 & 0.1523 & 0.1561 & 2.47 & 0.1929 & 0.1875 & 2.80 \\
\hline 150 & 0.4356 & 0.4305 & 1.18 & 0.0820 & 0.0738 & 10.04 & 0.1553 & 0.1512 & 2.64 \\
\hline 157.5 & 0.3542 & 0.3396 & 4.12 & 0.0360 & 0.0394 & 9.58 & 0.1171 & 0.1136 & 2.95 \\
\hline 165 & 0.2501 & 0.2480 & 0.85 & 0.0110 & 0.0058 & 46.98 & 0.0783 & 0.0760 & 2.92 \\
\hline 172.5 & 0.1294 & 0.1236 & 4.51 & 0.0014 & 0.0028 & 99.86 & 0.0392 & 0.0378 & 3.67 \\
\hline 180 & 0.0000 & 0.0001 & Inf. & 0.0000 & -0.0016 & Inf. & 0.0000 & -0.0006 & Inf. \\
\hline
\end{tabular}


Table 5.5 Comparison of stress state at mid-plane between finite element results and formulae evaluation for $a / W=0.8, t / W=2.0$, Uniform Load, SECP

\begin{tabular}{|c|c|c|c|c|c|c|c|c|c|}
\hline $\begin{array}{c}\text { Degree } \\
()\end{array}$ & \multicolumn{3}{|c|}{$f_{11}(\theta)$} & \multicolumn{3}{|c|}{$f_{22}(\theta)$} & \multicolumn{3}{|c|}{$f_{33}(\theta)$} \\
\hline & Eq.(5.2) & FEM & $\%$ & Eq.(5.2) & FEM & $\%$ & Eq.(5.2) & FEM & $\%$ \\
\hline 0 & 1.0000 & 0.9825 & 1.75 & 1.0000 & 0.9641 & 3.59 & 0.6000 & 0.5840 & 2.67 \\
\hline 7.5 & 0.9851 & 0.9529 & 3.27 & 1.0106 & 0.9854 & 2.50 & 0.5987 & 0.5815 & 2.88 \\
\hline 15 & 0.9419 & 0.9225 & 2.06 & 1.0410 & 1.0075 & 3.22 & 0.5949 & 0.5790 & 2.67 \\
\hline 22.5 & 0.8745 & 0.8438 & 3.51 & 1.0871 & 1.0614 & 2.36 & 0.5885 & 0.5716 & 2.87 \\
\hline 30 & 0.7891 & 0.7647 & 3.10 & 1.1427 & 1.1159 & 2.35 & 0.5796 & 0.5642 & 2.65 \\
\hline 37.5 & 0.6938 & 0.6655 & 4.08 & 1.2000 & 1.1743 & 2.14 & 0.5682 & 0.5520 & 2.85 \\
\hline 45 & 0.5972 & 0.5664 & 5.16 & 1.2505 & 1.2327 & 1.43 & 0.5543 & 0.5397 & 2.63 \\
\hline 52.5 & 0.5078 & 0.4814 & 5.20 & 1.2859 & 1.2616 & 1.89 & 0.5381 & 0.5229 & 2.83 \\
\hline 60 & 0.4330 & 0.3969 & 8.33 & 1.2990 & 1.2900 & 0.70 & 0.5196 & 0.5061 & 2.61 \\
\hline 67.5 & 0.3784 & 0.3526 & 6.81 & 1.2845 & 1.2637 & 1.62 & 0.4989 & 0.4849 & 2.80 \\
\hline 75 & 0.3472 & 0.3091 & 10.95 & 1.2396 & 1.2367 & 0.23 & 0.4760 & 0.4637 & 2.58 \\
\hline 82.5 & 0.3397 & 0.3132 & 7.78 & 1.1640 & 1.1487 & 1.31 & 0.4511 & 0.4386 & 2.77 \\
\hline 90 & 0.3536 & 0.3181 & 10.03 & 1.0607 & 1.0600 & 0.06 & 0.4243 & 0.4134 & 2.55 \\
\hline 97.5 & 0.3839 & 0.3564 & 7.17 & 0.9348 & 0.9260 & 0.94 & 0.3956 & 0.3847 & 2.75 \\
\hline 105 & 0.4239 & 0.3952 & 6.79 & 0.7936 & 0.7915 & 0.26 & 0.3653 & 0.3560 & 2.53 \\
\hline 112.5 & 0.4655 & 0.4377 & 5.96 & 0.6457 & 0.6430 & 0.42 & 0.3333 & 0.3242 & 2.74 \\
\hline 120 & 0.5000 & 0.4801 & 3.98 & 0.5000 & 0.4946 & 1.08 & 0.3000 & 0.2924 & 2.53 \\
\hline 127.5 & 0.5197 & 0.4937 & 5.00 & 0.3649 & 0.3666 & 0.46 & 0.2654 & 0.2581 & 2.75 \\
\hline 135 & 0.5180 & 0.5066 & 2.20 & 0.2474 & 0.2392 & 3.29 & 0.2296 & 0.2238 & 2.55 \\
\hline 142.5 & 0.4905 & 0.4689 & 4.42 & 0.1523 & 0.1561 & 2.46 & 0.1929 & 0.1875 & 2.78 \\
\hline 150 & 0.4356 & 0.4303 & 1.22 & 0.0820 & 0.0738 & 10.05 & 0.1553 & 0.1512 & 2.61 \\
\hline 157.5 & 0.3542 & 0.3394 & 4.16 & 0.0360 & 0.0394 & 9.57 & 0.1171 & 0.1137 & 2.89 \\
\hline 165 & 0.2501 & 0.2478 & 0.90 & 0.0110 & 0.0058 & 46.99 & 0.0783 & 0.0761 & 2.83 \\
\hline 172.5 & 0.1294 & 0.1235 & 4.58 & 0.0014 & 0.0028 & 99.84 & 0.0392 & 0.0379 & 3.45 \\
\hline 180 & 0.0000 & 0.0000 & Inf. & 0.0000 & -0.0016 & Inf. & 0.0000 & -0.0005 & Inf. \\
\hline & & & & & & & & & \\
\hline
\end{tabular}


Table 5.6 Comparison of stress state at mid-plane between finite element results and formulae evaluation for $a / W=0.8, t / W=4.0$, Uniform Load, SECP

\begin{tabular}{|c|c|c|c|c|c|c|c|c|c|}
\hline $\begin{array}{c}\text { Degree } \\
()\end{array}$ & \multicolumn{3}{|c|}{$f_{11}(\theta)$} & \multicolumn{3}{|c|}{$f_{22}(\theta)$} & \multicolumn{3}{|c|}{$f_{33}(\theta)$} \\
\hline 0 & Eq.(5.2) & FEM & $\%$ & Eq.(5.2) & FEM & $\%$ & Eq.(5.2) & FEM & $\%$ \\
\hline 7.5 & 0.9000 & 0.9825 & 1.75 & 1.0000 & 0.9641 & 3.59 & 0.6000 & 0.5840 & 2.67 \\
\hline 15 & 0.9419 & 0.9529 & 3.27 & 1.0106 & 0.9854 & 2.50 & 0.5987 & 0.5815 & 2.88 \\
\hline 22.5 & 0.8745 & 0.8438 & 3.50 & 1.0471 & 1.0075 & 3.21 & 0.5949 & 0.5790 & 2.67 \\
\hline 30 & 0.7891 & 0.7647 & 3.10 & 1.1427 & 1.1159 & 2.34 & 0.5796 & 0.5642 & 2.65 \\
\hline 37.5 & 0.6938 & 0.6655 & 4.08 & 1.2000 & 1.1743 & 2.14 & 0.5682 & 0.5520 & 2.85 \\
\hline 45 & 0.5972 & 0.5664 & 5.16 & 1.2505 & 1.2327 & 1.42 & 0.5543 & 0.5397 & 2.63 \\
\hline 52.5 & 0.5078 & 0.4814 & 5.20 & 1.2859 & 1.2616 & 1.89 & 0.5381 & 0.5229 & 2.83 \\
\hline 60 & 0.4330 & 0.3969 & 8.33 & 1.2990 & 1.2900 & 0.70 & 0.5196 & 0.5061 & 2.61 \\
\hline 67.5 & 0.3784 & 0.3526 & 6.82 & 1.2845 & 1.2637 & 1.62 & 0.4989 & 0.4849 & 2.80 \\
\hline 75 & 0.3472 & 0.3091 & 10.95 & 1.2396 & 1.2367 & 0.23 & 0.4760 & 0.4637 & 2.58 \\
\hline 82.5 & 0.3397 & 0.3132 & 7.79 & 1.1640 & 1.1487 & 1.31 & 0.4511 & 0.4386 & 2.77 \\
\hline 90 & 0.3536 & 0.3181 & 10.03 & 1.0607 & 1.0601 & 0.06 & 0.4243 & 0.4134 & 2.55 \\
\hline 97.5 & 0.3839 & 0.3564 & 7.17 & 0.9348 & 0.9260 & 0.94 & 0.3956 & 0.3847 & 2.75 \\
\hline 105 & 0.4239 & 0.3951 & 6.79 & 0.7936 & 0.7915 & 0.26 & 0.3653 & 0.3560 & 2.53 \\
\hline 112.5 & 0.4655 & 0.4377 & 5.96 & 0.6457 & 0.6430 & 0.42 & 0.3333 & 0.3242 & 2.74 \\
\hline 120 & 0.5000 & 0.4801 & 3.98 & 0.5000 & 0.4946 & 1.08 & 0.3000 & 0.2924 & 2.53 \\
\hline 127.5 & 0.5197 & 0.4937 & 5.01 & 0.3649 & 0.3666 & 0.47 & 0.2654 & 0.2581 & 2.75 \\
\hline 135 & 0.5180 & 0.5066 & 2.20 & 0.2474 & 0.2393 & 3.29 & 0.2296 & 0.2238 & 2.55 \\
\hline 142.5 & 0.4905 & 0.4689 & 4.42 & 0.1523 & 0.1561 & 2.46 & 0.1929 & 0.1875 & 2.79 \\
\hline 150 & 0.4356 & 0.4303 & 1.22 & 0.0820 & 0.0738 & 10.05 & 0.1553 & 0.1512 & 2.62 \\
\hline 157.5 & 0.3542 & 0.3394 & 4.17 & 0.0360 & 0.0394 & 9.57 & 0.1171 & 0.1137 & 2.90 \\
\hline 165 & 0.2501 & 0.2478 & 0.90 & 0.0110 & 0.0058 & 46.99 & 0.0783 & 0.0761 & 2.84 \\
\hline 172.5 & 0.1294 & 0.1235 & 4.58 & 0.0014 & 0.0028 & 99.85 & 0.0392 & 0.0379 & 3.47 \\
\hline 180 & 0.0000 & 0.0000 & Inf. & 0.0000 & -0.0016 & Inf. & 0.0000 & -0.0005 & Inf. \\
\hline
\end{tabular}


Table 5.7 Percent different between Williams formula and FEM, $a / W=0.6, t / W=0.5$, Uniform Load, SECP

\begin{tabular}{|c|c|c|c|c|c|c|c|c|c|}
\hline \multirow{2}{*}{$r / a$} & \multicolumn{3}{|c|}{$0^{\circ}(\%)$} & \multicolumn{3}{|c|}{$30^{\circ}(\%)$} & \multicolumn{3}{|c|}{$60^{\circ}(\%)$} \\
\cline { 2 - 11 } & $f_{11}$ & $f_{22}$ & $f_{33}$ & $f_{11}$ & $f_{22}$ & $f_{33}$ & $f_{11}$ & $f_{22}$ & $f_{33}$ \\
\hline 0.0044 & 1.84 & 3.56 & 1.64 & 3.03 & 2.54 & 1.61 & 7.74 & 1.30 & 1.52 \\
\hline 0.0054 & 2.12 & 3.85 & 1.82 & 3.44 & 2.77 & 1.79 & 8.62 & 1.46 & 1.70 \\
\hline 0.0066 & 2.45 & 4.20 & 2.03 & 3.91 & 3.04 & 2.00 & 9.56 & 1.67 & 1.91 \\
\hline 0.0079 & 2.82 & 4.58 & 2.26 & 4.42 & 3.35 & 2.23 & 10.56 & 1.90 & 2.13 \\
\hline 0.0094 & 3.22 & 5.00 & 2.52 & 4.95 & 3.68 & 2.48 & 11.60 & 2.17 & 2.37 \\
\hline 0.0109 & 3.65 & 5.44 & 2.79 & 5.51 & 4.05 & 2.75 & 12.68 & 2.44 & 2.62 \\
\hline 0.0126 & 4.10 & 5.92 & 3.08 & 6.10 & 4.44 & 3.04 & 13.81 & 2.73 & 2.89 \\
\hline 0.0144 & 4.58 & 6.43 & 3.40 & 6.73 & 4.86 & 3.34 & 15.04 & 3.04 & 3.18 \\
\hline 0.0164 & 5.09 & 6.97 & 3.73 & 7.41 & 5.29 & 3.67 & 16.31 & 3.37 & 3.48 \\
\hline 0.0234 & 6.82 & 8.82 & 4.88 & 9.66 & 6.82 & 4.79 & 20.63 & 4.49 & 4.50 \\
\hline \multirow{2}{*}{$r / a$} & & $90^{\circ}(\%)$ & & & $120^{\circ}(\%)$ & & $150^{\circ}(\%)$ & \\
\cline { 2 - 12 } & $f_{11}$ & $f_{22}$ & $f_{33}$ & $f_{11}$ & $f_{22}$ & $f_{33}$ & $f_{11}$ & $f_{22}$ & $f_{33}$ \\
\hline 0.0044 & 9.41 & 1.30 & 1.41 & 4.26 & 4.58 & 1.31 & 3.21 & 34.18 & 1.25 \\
\hline 0.0054 & 10.45 & 1.51 & 1.59 & 4.94 & 5.04 & 1.48 & 3.92 & 36.98 & 1.40 \\
\hline 0.0066 & 11.56 & 1.77 & 1.79 & 5.68 & 5.56 & 1.67 & 4.66 & 39.79 & 1.56 \\
\hline 0.0079 & 12.74 & 2.04 & 2.00 & 6.46 & 6.08 & 1.85 & 5.42 & 42.59 & 1.72 \\
\hline 0.0094 & 14.02 & 2.32 & 2.22 & 7.28 & 6.60 & 2.04 & 6.20 & 45.39 & 1.88 \\
\hline 0.0109 & 15.32 & 2.61 & 2.45 & 8.14 & 7.12 & 2.24 & 7.02 & 48.32 & 2.04 \\
\hline 0.0126 & 16.73 & 2.91 & 2.69 & 9.04 & 7.66 & 2.45 & 7.87 & 51.12 & 2.20 \\
\hline 0.0144 & 18.20 & 3.23 & 2.94 & 10.00 & 8.20 & 2.66 & 8.77 & 54.17 & 2.37 \\
\hline 0.0164 & 19.76 & 3.55 & 3.21 & 11.02 & 8.76 & 2.88 & 9.71 & 57.10 & 2.55 \\
\hline 0.0234 & 25.02 & 4.63 & 4.09 & 14.44 & 10.58 & 3.59 & 12.85 & 66.60 & 3.08 \\
\hline
\end{tabular}


Table 5.8 Stress State along the circular path close to the front varied along thickness, $a / W=0.6, t / W=0.1, r / a=0.0078$, Uniform, SECP

\begin{tabular}{|c|c|c|c|c|c|c|c|c|c|c|c|c|c|c|c|}
\hline \multirow{2}{*}{$\theta\left({ }^{\circ}\right)$} & \multicolumn{5}{|c|}{$f_{11}(\theta)$} & \multicolumn{5}{|c|}{$f_{22}(\theta)$} & \multicolumn{5}{|c|}{$f_{33}(\theta)$} \\
\hline & Eq.(5.2) & $z / t=0$ & 0.1311 & 0.2702 & 0.3861 & Eq.(5.2) & $z / t=0$ & 0.1311 & 0.2702 & 0.3861 & Eq.(5.2) & $z / t=0$ & 0.1311 & 0.2702 & 0.3861 \\
\hline 0 & 1.0000 & \begin{tabular}{|l|}
0.9668 \\
\end{tabular} & 0.9661 & 0.9619 & 0.9434 & 1.0000 & 0.9468 & 0.9459 & 0.9408 & 0.9231 & 0.6000 & 0.5629 & 0.5607 & 0.5505 & 0.5248 \\
\hline 7.5 & 0.9851 & 0.9371 & 0.9364 & 0.9322 & 0.9139 & 1.0106 & 0.9680 & 0.9670 & 0.9620 & .9443 & 5987 & 0.5605 & 584 & 0.5484 & 0.5232 \\
\hline 15 & 0.9419 & 0.9067 & 0.9060 & 0.9018 & 0.8835 & 1.0410 & 0.9900 & 0.9891 & 0.9841 & 0.9664 & 0.5949 & 0.5582 & 0.5561 & 0.5463 & 0.5216 \\
\hline 22.5 & 0.8745 & 0.8279 & 0.8272 & 0.8230 & 0.8049 & 871 & 1.0436 & 1.0427 & 1.0378 & 1.0203 & 0.5885 & 0.5513 & 0.5493 & 0.5400 & 0.5169 \\
\hline 30 & 0.7891 & 0.7487 & 0.7480 & 0.7437 & 0.7258 & 1.1427 & 1.0977 & 1.0968 & 1.0920 & 1.0747 & 0.5796 & 0.5444 & 0.5425 & 0.5337 & 0.5121 \\
\hline 37.5 & 0.6938 & 0.6494 & 0.6487 & 0.6443 & 0.6265 & 1.2000 & 1.1554 & 1.1546 & 1.1500 & 1.1331 & 682 & 0.5329 & 312 & 0.5232 & 0.5043 \\
\hline 45 & 0.5972 & 0.5502 & 0.5495 & 0.5450 & 0.5273 & 1.2505 & 1.2132 & 1.2124 & 1.2080 & 1.1915 & 0.5543 & 0.5215 & 0.5199 & 0.5127 & 0.4964 \\
\hline 52.5 & 0.5078 & 0.4651 & 0.4643 & 0.4596 & 0.4419 & 1.2859 & 1.2412 & 1.2405 & 1.2363 & 1.2207 & 0.5381 & 0.5056 & 0.5043 & 0.4982 & 0.4854 \\
\hline 60 & 0.4330 & 0.3805 & 0.3796 & 0.3749 & 0.3571 & 1.2990 & 1.2688 & 1.2681 & 1.2642 & 1.2493 & 0.5196 & 0.4898 & 0.4887 & 0.4837 & 0.4744 \\
\hline 67.5 & 0.3784 & 0.3360 & 0.3351 & 0.3301 & 0.3120 & 1.2845 & 1.2415 & 1.2409 & 1.2373 & 1.2237 & 0.4989 & 0.4698 & 0.4690 & 0.4653 & 0.4603 \\
\hline 75 & 0.3472 & 0.2923 & 0.2913 & 0.2862 & 0.2678 & 1.2396 & 1.2135 & 1.2129 & 1.2097 & 72 & 760 & 0.4499 & 493 & 469 & 462 \\
\hline 82.5 & 0.3397 & 0.2960 & 0.2950 & 0.2897 & 0.2708 & 1.1640 & 1.1245 & 1.1240 & 1.1212 & 1.1103 & 0.4511 & 0.4260 & 0.4257 & 4248 & 0.4291 \\
\hline 90 & 0.3536 & 0.3005 & 0.2994 & 0.2939 & 0.2745 & 6007 & 1.0349 & 1.0345 & 1.0320 & .0226 & 43 & 0.4021 & 021 & 026 & 119 \\
\hline 97.5 & 0.3839 & 0.3381 & 0.3371 & 0.3314 & 0.3114 & 0.9348 & 0.9000 & 0.8996 & 0.8976 & 0.8898 & 0.3956 & 0.3746 & 0.3749 & 0.3770 & 0.3917 \\
\hline 105 & 0.4239 & 0.3762 & 0.3751 & 0.3693 & 0.3487 & 0.7936 & 0.7648 & 0.7645 & 0.7628 & 0.7566 & 0.3653 & 0.3471 & 0.3478 & 0.3514 & 0.3714 \\
\hline 112.5 & 0.4655 & 0.4178 & 0.4167 & 0.4108 & 0.3899 & 0.6457 & 0.6157 & 0.6154 & 0.6141 & 0.6092 & 0.3333 & 0.3165 & 0.3174 & 0.3226 & 0.3481 \\
\hline 120 & 0.5000 & 0.4593 & 0.4581 & 0.4522 & 0.4309 & 0.5000 & 0.4667 & 0.4666 & 0.4656 & 0.4621 & 0.3000 & 0.2858 & 0.2871 & 0.2938 & 0.3247 \\
\hline 127.5 & 0.5197 & 0.4716 & 0.4705 & 0.4646 & 0.4435 & 0.3649 & 0.3384 & 0.3383 & 0.3375 & 0.3350 & 0.2654 & 0.2524 & 0.2539 & 0.2620 & 0.2982 \\
\hline 135 & 0.5180 & 0.4834 & 0.4823 & 0.4765 & 0.4555 & 0.2474 & 0.2107 & 0.2106 & 0.2101 & 0.2085 & 0.2296 & 0.2190 & 0.2208 & 0.2302 & 0.2716 \\
\hline 142.5 & 0.4905 & 0.4443 & 0.4433 & 0.4377 & 0.4175 & 0.1523 & 0.1274 & 0.1273 & 0.1269 & 0.1258 & 0.1929 & 0.1833 & 0.1853 & 0.1958 & 0.2417 \\
\hline 150 & 0.4356 & 0.4044 & 0.4034 & 0.3982 & 0.3787 & 0.0820 & 0.0450 & 0.0449 & 0.0446 & 0.0440 & 0.1553 & 0.1476 & 0.1498 & 0.1615 & 0.2119 \\
\hline 157.5 & 0.3542 & 0.3122 & 0.3113 & 0.3065 & 0.2883 & 0.0360 & 0.0105 & 0.0105 & 0.0102 & 0.0096 & 0.1171 & 0.1101 & 0.1125 & 0.1249 & 0.1784 \\
\hline 165 & 0.2501 & 0.2192 & 0.2184 & 0.2141 & 0.1973 & 0.0110 & -0.0232 & -0.0232 & -0.0235 & -0.0240 & 0.0783 & 0.0727 & 0.0752 & 0.0883 & 0.1450 \\
\hline 172.5 & 0.1294 & 0.0935 & 0.0928 & 0.0891 & 0.0743 & 0.0014 & -0.0262 & -0.0263 & -0.0265 & -0.0271 & 0.0392 & 0.0341 & 0.0366 & 0.0500 & 0.1079 \\
\hline 180 & 0.0000 & -0.0313 & -0.0319 & -0.0350 & -0.0478 & 0.0000 & -0.0307 & -0.0307 & -0.0310 & -0.0316 & 0.0000 & -0.0046 & -0.0020 & 0.0115 & 0.0706 \\
\hline
\end{tabular}


Table 5.9 Stress State along the circular path close to the front varied along thickness, $a / W=0.6, t / W=0.2, r / a=0.0078$, Uniform, SECP

\begin{tabular}{|c|c|c|c|c|c|c|c|c|c|c|c|c|c|c|c|}
\hline \multirow{2}{*}{$\theta\left(^{\circ}\right)$} & \multicolumn{5}{|c|}{$f_{11}(\theta)$} & \multicolumn{5}{|c|}{$f_{22}(\theta)$} & \multicolumn{5}{|c|}{$f_{33}(\theta)$} \\
\hline & Eq.(5.2) & $z / t=0$ & 0.1228 & 0.2627 & 0.4244 & Eq.(5.2) & $z / t=0$ & 0.1228 & 0.2627 & 0.4244 & Eq.(5.2) & $z / t=0$ & 0.1228 & 0.2627 & 0.4244 \\
\hline 0 & 1.0000 & 0.9674 & 0.9671 & 0.9650 & 0.9464 & 1.0000 & 0.9490 & 0.9485 & 0.9459 & 0.9254 & 0.6000 & 0.5713 & 0.5704 & 0.5659 & 0.5330 \\
\hline 7.5 & 0.9851 & 0.9378 & 0.9374 & 0.9353 & 0.9168 & 1.0106 & 0.9702 & 0.9697 & 0.9671 & 0.9467 & 0.5987 & 0.5688 & 0.5679 & 0.5635 & 0.5312 \\
\hline 15 & 0.9419 & 0.9075 & 0.9071 & 0.9049 & 0.8863 & 10 & 9922 & 17 & 91 & 39 & 949 & 663 & 655 & 612 & 293 \\
\hline 22.5 & 0.8745 & 0.8288 & 0.8284 & 3262 & 075 & 71 & 57 & 152 & 127 & 29 & 385 & 590 & 582 & 540 & 238 \\
\hline 30 & - & 0.7496 & 0.7492 & 0.7470 & 0.7282 & 1.1427 & 1.008 & 1.0992 & 1.0968 & 1.0774 & 0.5796 & 0.5517 & 0.5509 & 0.5469 & 0.5183 \\
\hline 37.5 & 0.6938 & 0.6505 & 0.6501 & 0.6478 & 0.6288 & & 1.1574 & & 546 & & 682 & 0.5395 & 388 & 352 & 6091 \\
\hline 45 & 0.5972 & 0.5514 & 0.5510 & 0.5487 & 0.5294 & 05 & 1.2151 & 1.2146 & 1.2124 & 946 & 543 & 274 & 268 & 234 & 1999 \\
\hline 52.5 & 0.5078 & 0.4665 & 0.4660 & 0.4636 & 0.4440 & 59 & 1.2430 & 1.2425 & 1.2405 & 238 & 381 & 0.5107 & 102 & 072 & 4872 \\
\hline 60 & 0.4330 & 0.3821 & \begin{tabular}{|l|}
0.3816 \\
\end{tabular} & 0.3791 & 0.3591 & 1.2990 & 1.2704 & 1.2700 & 1.2681 & 1.2525 & 0.5196 & 0.4941 & 0.4936 & 0.4910 & 0.4744 \\
\hline 67.5 & 0.3784 & 0.3377 & 0.3373 & 0.3347 & 0.3142 & 1.2845 & 1.2429 & 1.2426 & 1.2409 & 1.2267 & 0.4989 & 0.4730 & 0.4726 & 0.4705 & 0.4581 \\
\hline 75 & 0.3472 & 0.2943 & 0.2938 & 0.2911 & 0.2702 & 1.2396 & 1.2147 & 1.2144 & 1.2129 & 1.2001 & 0.4760 & 0.4520 & 0.4517 & 501 & 0.4419 \\
\hline 82.5 & 0.3397 & 0.2982 & 0.2977 & 0.2950 & 0.2735 & 40 & 257 & 254 & 240 & 27 & 511 & 270 & 268 & 257 & 222 \\
\hline 90 & 0 & 9 & 4 & 0 & 0 & 7 & 8 & 6 & 4 & 7 & 3 & 20 & 19 & 3 & 326 \\
\hline 97.5 & & & 0 & & 0. & & 8 & 06 & 196 & 14 & 56 & 34 & 734 & & 3796 \\
\hline 105 & 0.4239 & 0.3790 & 0.3785 & 0.3757 & 0.3530 & & 0.7654 & 0.7652 & 0.7644 & 77 & 0.3653 & 488 & 48 & 154 & 567 \\
\hline 112.5 & 0.4655 & 0.4208 & 0.4203 & 0.4175 & 0.3947 & 0.6457 & 0.6162 & 0.6160 & 0.6153 & 0.6099 & 0.3333 & 0.3130 & 0.3132 & 0.3142 & 0.3305 \\
\hline 120 & 0.5000 & 0.4624 & 0.4619 & 0.4591 & 0.4364 & 0.5000 & 0.4671 & 0.4670 & 0.4664 & 0.4623 & 0.3000 & 0.2812 & 0.2815 & 0.2831 & 0.3043 \\
\hline 127.5 & 0.5197 & 0.4748 & 0.4743 & 0.4717 & 0.4495 & 0.3649 & 0.3387 & 0.3385 & 0.3381 & 0.3349 & 0.2654 & 0.2469 & 0.2472 & 0.2492 & 0.2751 \\
\hline 135 & 0.5180 & 0.4866 & 0.4861 & 0.4836 & 0.4619 & 0.2474 & 0.2109 & 0.2108 & 0.2104 & 0.2081 & 0.2296 & 0.2125 & 0.2129 & 0.2153 & 0.2458 \\
\hline 142.5 & 0.4905 & 0.4475 & 0.4470 & 0.4447 & 0.4242 & 0.1523 & 0.1275 & 0.1274 & 0.1271 & 0.1253 & 0.1929 & 0.1760 & 0.1765 & 0.1793 & 0.2135 \\
\hline 150 & 0.4356 & 0.4075 & 0.4071 & 0.4049 & 0.3857 & 0.0820 & 0.0450 & 0.0449 & 0.0446 & 0.0433 & 0.1553 & 0.1396 & 0.1401 & 0.1433 & 0.1813 \\
\hline 157.5 & 0.3542 & 0.3151 & 0.3147 & 0.3128 & 0.2954 & 0.0360 & 0.0105 & 0.0105 & 0.0102 & 0.0089 & 0.1171 & 0.1017 & 0.1023 & 0.1057 & 0.1462 \\
\hline 165 & 0.2501 & 0.2220 & 0.2217 & 0.2200 & 0.2045 & 0.0110 & -0.0232 & -0.0233 & -0.0235 & -0.0246 & 0.0783 & 0.0638 & 0.0644 & 0.0680 & 0.1111 \\
\hline 172.5 & 0.1294 & 0.0960 & 0.0957 & 0.0944 & 0.0813 & 0.0014 & -0.0263 & -0.0263 & -0.0266 & -0.0277 & 0.0392 & 0.0251 & 0.0257 & 0.0294 & 0.0733 \\
\hline 180 & 0.0000 & 0291 & .0293 & .0304 & .0410 & 0.0000 & -0.0307 & -0.0308 & 0.0310 & -0.0322 & 0.0000 & -0.0138 & .0131 & 0.0093 & .0354 \\
\hline
\end{tabular}


Table 5.10 Stress State along the circular path close to the front varied along thickness, $a / W=0.6, t / W=0.5, r / a=0.0078$, Uniform, SECP

\begin{tabular}{|c|c|c|c|c|c|c|c|c|c|c|c|c|c|c|c|}
\hline \multirow{2}{*}{$\theta\left({ }^{\circ}\right)$} & \multicolumn{5}{|c|}{$f_{11}(\theta)$} & \multicolumn{5}{|c|}{$f_{22}(\theta)$} & \multicolumn{5}{|c|}{$f_{33}(\theta)$} \\
\hline & Eq.(5.2) & $z / t=0$ & 0.1161 & 0.2637 & 0.4182 & Eq.(5.2) & $z / t=0$ & 0.1161 & 0.2637 & 0.4182 & Eq.(5.2) & $z / t=0$ & 0.1161 & 0.2637 & 0.4182 \\
\hline 0 & 1.0000 & 0.9974 & 0.9677 & 0.9665 & 0.9592 & 1.0000 & 0.9796 & 0.9498 & 0.9486 & 0.9401 & 0.6000 & 0.5926 & 0.5746 & 0.5731 & 0.5618 \\
\hline 7.5 & 0.9851 & 0.9678 & 0.9381 & 0.9369 & 0.9295 & 1.0106 & 1.0008 & 0.9710 & 0.9697 & 0.9614 & 0.5987 & 0.5901 & 0.5721 & 0.5705 & 0.5594 \\
\hline 15 & 0.9419 & 0.9374 & 0.9077 & 0.9066 & 0.8991 & 1.0410 & 1.0227 & 0.9930 & 0.9917 & 0.9834 & 0.5949 & 0.5876 & 0.5696 & 0.5681 & 0.5571 \\
\hline 22.5 & 0.8745 & 0.8588 & 0.8291 & 0.8279 & 0.8203 & 1.0871 & 1.0762 & 1.0464 & 1.0452 & 1.0372 & 0.5885 & 0.5800 & 0.5621 & 0.5606 & 0.5500 \\
\hline 30 & 0.7891 & 0.7796 & 0.7500 & 0.7488 & 0.7411 & 1.1427 & .1302 & 1.1004 & 1.0993 & 1.0915 & 0.5796 & 0.5725 & 0.5546 & 0.5532 & 0.5430 \\
\hline 37.5 & 938 & 6805 & 0.6509 & 0.6497 & 0.6418 & 1.2000 & 1879 & 581 & 569 & 2 & 682 & 5601 & 422 & 409 & 314 \\
\hline 45 & 0.5972 & .5815 & 0.5519 & 0.5507 & 0.5427 & 1.2505 & 1.0700 & 1.2157 & 1.2146 & 1.60 & 0.5543 & 0.5478 & (.)- & 0.5286 & 0.5198 \\
\hline 52.5 & 078 & 0.4966 & 0.4670 & 0.4658 & 0.4575 & 859 & 1.2733 & 1.270 & 425 & 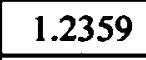 & 381 & 0.5307 & 128 & 117 & 038 \\
\hline 60 & 30 & 23 & 0.3 & 14 & 0 & 90 & 07 & 08 & 599 & 38 & 196 & 136 & 958 & 948 & 4878 \\
\hline 67.5 & 0.3784 & 0.3680 & 0. & 0 & 0 & 45 & 32 & 34 & 25 & 59 & 89 & 922 & 743 & 735 & 0.4676 \\
\hline 75 & 0.3472 & 0.3246 & 0.2951 & 0.2938 & 0.2850 & 1.2396 & 1.2449 & 1.2151 & 1.2142 & 1.2092 & 0.4760 & 0.4708 & 0.4529 & 0.4522 & 0.4474 \\
\hline 82.5 & 0.3397 & 0.3286 & 0.2991 & 0.2978 & 0.2889 & 1.1640 & 1.1556 & 1.1259 & 1.1251 & 1.1207 & 0.4511 & 0.4453 & 0.4275 & 0.4269 & 0.4232 \\
\hline 90 & 0.3536 & 0.3334 & 0.3039 & 0.3025 & 0.2936 & 1.0607 & 1.0657 & 1.0360 & 1.0353 & 1.0314 & 0.4243 & 0.4198 & 0.4020 & 0.4016 & 0.3991 \\
\hline 97.5 & 0.3839 & 0.3714 & 0.3418 & 0.3405 & 0.3316 & 0.9348 & 0.9305 & 0.9009 & 0.9003 & 0.8968 & 0.3956 & 0.3907 & 0.3730 & 0.3726 & 0.3714 \\
\hline 105 & 0.4239 & 0.4097 & 0.3801 & 0.3788 & 0.3699 & 0.7936 & 0.7950 & 0.7654 & 0.7649 & 0.7619 & 0.3653 & 0.3616 & 0.3439 & 0.3437 & 0.3437 \\
\hline 112.5 & 0.4655 & 0.4515 & 0.4219 & 0.4206 & 0.4119 & 0.6457 & 0.6457 & 0.6161 & 0.6156 & 0.6130 & 0.3333 & 0.3294 & 0.3117 & 0.3117 & 0.3128 \\
\hline 120 & 0.5000 & 0.4931 & 0.4635 & 0.4622 & 0.4538 & 0.5000 & 0.4965 & 0.4670 & 0.4665 & 0.4643 & 0.3000 & 0.2972 & 0.2796 & 0.2796 & 0.2820 \\
\hline 127.5 & 0.5197 & 0.5055 & 0.4759 & 0.4747 & 0.4667 & 0.3649 & 0.3680 & 0.3385 & 0.3381 & 0.3362 & 0.2654 & 0.2624 & 0.2448 & 0.2450 & 0.2484 \\
\hline 135 & 0.5180 & 0.5173 & 0.4877 & 0.4866 & 0.4789 & 0.2474 & 0.2401 & 0.2107 & 0.2103 & 0.2087 & 0.2296 & 0.2277 & 0.2101 & 0.2104 & 0.2148 \\
\hline 142.5 & 0.4905 & 0.4781 & 0.4485 & 0.4475 & 0.4405 & 0.1523 & 0.1566 & 0.1273 & 0.1269 & 0.1254 & 0.1929 & 0.1909 & 0.1733 & 0.1738 & 0.1790 \\
\hline 150 & 0.4356 & 0.4380 & 0.4085 & 0.4075 & 0.4012 & 0.0820 & 0.0741 & 0.0447 & 0.0444 & 0.0430 & 0.1553 & 0.1542 & 0.1366 & 0.1371 & 0.1432 \\
\hline 157.5 & 0.3542 & 0.3454 & 0.3159 & 0.3151 & 0.3096 & 0.0360 & 0.0396 & 0.0103 & 0.0099 & 0.0086 & 0.1171 & 0.1161 & 0.0986 & 0.0991 & 0.1057 \\
\hline 165 & 0.2501 & 0.2522 & 0.2227 & 0.2220 & 0.2172 & 0.0110 & 0.0058 & -0.0235 & -0.0238 & -0.0250 & 0.0783 & 0.0780 & 0.0605 & 0.0611 & 0.0683 \\
\hline 172.5 & 0.1294 & 0.1260 & 0.0966 & 0.0960 & 0.0922 & 0.0014 & 0.0028 & -0.0265 & -0.0268 & -0.0281 & 0.0392 & 0.0392 & 0.0217 & 0.0224 & 0.0298 \\
\hline 180 & 0.0000 & 0.0007 & -0.0287 & -0.0291 & -0.0320 & 0.0000 & -0.0017 & -0.0310 & -0.0313 & -0.0326 & 0.0000 & 0.0003 & -0.0172 & -0.0165 & -0.0089 \\
\hline
\end{tabular}


Table 5.11 Stress State along the circular path close to the front varied along thickness, $a / W=0.6, t / W=1.0, r / a=0.0078$, Uniform, SECP

\begin{tabular}{|c|c|c|c|c|c|c|c|c|c|c|c|c|c|c|c|}
\hline \multirow{2}{*}{$\theta\left({ }^{\circ}\right)$} & \multicolumn{5}{|c|}{$f_{11}(\theta)$} & \multicolumn{5}{|c|}{$f_{22}(\theta)$} & \multicolumn{5}{|c|}{$f_{33}(\theta)$} \\
\hline & Eq.(5.2) & $z / t=0$ & 0.1161 & 0.2637 & 0.4182 & q.(5.2) & $z / t=0$ & 0.1161 & 2637 & 0.4182 & Eq.(5.2) & $z / t=0$ & 0.1161 & 0.2637 & 0.4182 \\
\hline 0 & 1.0000 & 0.9975 & 0.9673 & 0.9669 & 0.9631 & 1.0000 & 0.9799 & 0.9497 & 0.9492 & 0.9449 & 0.6000 & 0.5932 & 0.5751 & 0.5746 & 0.5699 \\
\hline 7.5 & 0.9851 & 9679 & 0.9377 & 0.9373 & .9334 & 1.0106 & 1.0010 & 0.9708 & 0.9704 & 0.9661 & .5987 & 0.5907 & 0.5726 & 0.5721 & .5675 \\
\hline 15 & 0.9419 & 375 & 0.9074 & 0.9070 & 0.9031 & 1.0410 & 1.0230 & 0.9928 & 0.9923 & 0.9881 & .5949 & 0.5882 & 5701 & 0.5696 & .5650 \\
\hline 22.5 & 8745 & 0.8588 & 0.8287 & 0.8283 & 0.8244 & 1.0871 & 1.0765 & 1.0463 & 1.0458 & 1.0417 & 5885 & 0.5806 & 5625 & 520 & .5576 \\
\hline 30 & 891 & 7797 & 0.7497 & 0.7493 & 0.7452 & 1.1427 & 1.1305 & 1.1003 & 1.0998 & 1.0958 & 5796 & 0.5731 & 5550 & .5545 & 5503 \\
\hline 37.5 & 9938 & 0.6806 & 0.6506 & 0.6502 & 0.6461 & 1.2000 & 1.1881 & 1.1579 & 1.1574 & 1536 & $\overline{5682}$ & .5607 & 5426 & 5421 & 5381 \\
\hline 45 & 0.5972 & 0.5816 & 0.5516 & 0.5512 & 0.5470 & 1.2505 & 1.2457 & 1.2155 & 1.2150 & 1.2113 & 0.5543 & 0.5482 & .5301 & 5297 & .5260 \\
\hline 52.5 & 0.5078 & 0.4967 & 0.4668 & 0.4663 & 0.4621 & 1.2859 & 1.2736 & 1.2433 & 1.2429 & 1.2394 & 0.5381 & 0.5311 & 5130 & .5126 & .5092 \\
\hline 60 & 0.4330 & 0.4124 & 0.3825 & 0.3820 & 0.3777 & 1.2990 & 1.3009 & 1.2706 & 1.2702 & 1.2669 & 0.5196 & 0.5140 & 0.4959 & 0.4956 & 4925 \\
\hline 67.5 & 0.3784 & 0.3682 & 0.3383 & 0.3378 & 0.3334 & 1.2845 & 1.2733 & 1.2431 & 1.2427 & 1.2397 & 0.4989 & 0.4925 & 0.4744 & 0.4741 & 0.4714 \\
\hline 75 & 0.3472 & 0.3248 & 0.2949 & 0.2944 & 0.2900 & 1.2396 & 1.2450 & 1.2148 & 1.2144 & 1.2116 & 4760 & 0.4710 & 4529 & 4526 & 4503 \\
\hline 82.5 & 0.3397 & 0.3289 & 0.2989 & 0.2985 & 0.2940 & 1.1640 & 1.1558 & 1.1256 & 1.1252 & 1.1227 & 4511 & .4454 & 4274 & $\overline{71}$ & 4252 \\
\hline 90 & 0.3536 & 0.3337 & 0.3038 & 0.3033 & 0.2987 & 1.0607 & 1.0658 & 1.0356 & 1.0353 & 1.0330 & 4243 & 0.4199 & .4018 & .4016 & .4001 \\
\hline 97.5 & 0.3839 & 0.3716 & 0.3417 & 0.3412 & 0.3367 & 0.9348 & 0.9307 & 0.9005 & 0.9002 & 0.8981 & 3956 & 0.3907 & .3727 & .3725 & .3714 \\
\hline 105 & 0.4239 & 0.4100 & 0.3800 & 0.3795 & 0.3751 & 0.7936 & 0.7951 & 0.7650 & 0.7647 & 0.7628 & 0.3653 & 0.3615 & 0.3435 & 0.3434 & 0.3428 \\
\hline 112.5 & 0.4655 & 0.4518 & 0.4218 & 0.4213 & 0.4170 & 0.6457 & 0.6457 & 0.6157 & 0.6154 & 0.6137 & 0.3333 & 0.3292 & 0.3113 & 0.3112 & 0.3109 \\
\hline 120 & 0.5000 & 0.4934 & 0.4634 & 0.4629 & 0.4587 & 0.5000 & 0.4965 & 0.4666 & 0.4663 & 0.4647 & 0.3000 & 0.2970 & 0.2790 & .2790 & 0.2791 \\
\hline 127.5 & 0.5197 & 0.5058 & 0.4758 & 0.4754 & 0.4713 & 0.3649 & 0.3680 & 0.3381 & 0.3378 & 0.3364 & 0.2654 & 0.2621 & 0.2442 & 0.2442 & .2447 \\
\hline 135 & 0.5180 & 0.5176 & 0.4876 & 0.4871 & 0.4833 & 0.2474 & 0.2401 & 0.2102 & 0.2100 & 0.2087 & 0.2296 & 0.2273 & 0.2094 & 0.2094 & 0.2103 \\
\hline 142.5 & 0.4905 & 0.4784 & 0.4484 & 0.4480 & 0.4444 & 0.1523 & 0.1567 & 0.1268 & 0.1266 & 0.1253 & 0.1929 & 0.1905 & 0.1726 & 0.1726 & 0.1738 \\
\hline 150 & 0.4356 & 0.4383 & 0.4084 & 0.4079 & 0.4047 & 0.0820 & 0.0741 & 0.0443 & 0.0440 & 0.0428 & 0.1553 & 0.1537 & 0.1358 & 0.1359 & 0.1373 \\
\hline 157.5 & 0.3542 & 0.3457 & 0.3158 & 0.3154 & 0.3126 & 0.0360 & 0.0396 & 0.0098 & 0.0096 & 0.0084 & 0.1171 & 0.1156 & 0.0977 & 0.0978 & $\overline{0.0995}$ \\
\hline 165 & 0.2501 & 0.2524 & 0.2225 & 0.2222 & 0.2197 & 0.0110 & 0.0058 & -0.0239 & -0.0242 & -0.0253 & 0.0783 & 0.0775 & 0.0596 & .0597 & 0.0616 \\
\hline 172.5 & 0.1294 & 0.1262 & 0.0964 & 0.0960 & 0.0940 & 0.0014 & 0.0028 & -0.0270 & -0.0272 & -0.0284 & 0392 & 0.0387 & 0.0208 & 0.0209 & 0.0229 \\
\hline 180 & 0.0000 & 0.0008 & .0289 & 0.0292 & .0309 & 0.0000 & -0.0017 & -0.0315 & -0.0317 & -0.0328 & .0000 & -0.0003 & -0.0181 & -0.0180 & -0.0159 \\
\hline
\end{tabular}


Table 5.12 Stress State along the circular path close to the front varied along thickness, $a / W=0.6, t / W=2.0, r / a=0.0078$, Uniform, SECP

\begin{tabular}{|c|c|c|c|c|c|c|c|c|c|c|c|c|c|c|c|}
\hline \multirow{2}{*}{$\theta\left({ }^{\circ}\right)$} & \multicolumn{5}{|c|}{$f_{11}(\theta)$} & \multicolumn{5}{|c|}{$f_{22}(\theta)$} & \multicolumn{5}{|c|}{$f_{33}(\theta)$} \\
\hline & Eq.(5.2) & $z / t=0$ & 0.1161 & 0.2637 & 0.4182 & Eq.(5.2) & $z / t=0$ & 0.1161 & 0.2637 & 0.4182 & Eq.(5.2) & $z / t=0$ & 0.1161 & 0.2637 & 0.4182 \\
\hline 0 & 1.0000 & 0.9974 & 0.9667 & 0.9668 & 0.9655 & 1.0000 & 0.9798 & 0.9491 & 0.9492 & 0.9477 & 0.6000 & 0.5932 & 0.5748 & 0.5748 & 0.5734 \\
\hline 7.5 & 0.9851 & 0.9678 & 0.9371 & 0.9372 & 0.9359 & 1.0106 & 1.0009 & 0.9703 & 0.9703 & 0.9688 & 0.5987 & 0.5906 & 0.5722 & 0.5723 & 0.5709 \\
\hline 15 & 0.9419 & 0.9374 & 0.9068 & 0.9068 & 0.9056 & 1.0410 & 1.0229 & 0.9922 & 0.9923 & 908 & 949 & 881 & 697 & 98 & 5684 \\
\hline 22.5 & 0.8745 & 0.8588 & 0.8281 & 0.8282 & 0.8269 & 8871 & .0764 & 1.0457 & 457 & 143 & 885 & 0.5806 & 622 & 622 & 5609 \\
\hline 30 & 91 & 0.7796 & 491 & 0.7491 & 8 & 7 & 4 & 1 & 7 & 3 & 96 & 730 & 17 & 17 & 534 \\
\hline 37.5 & 0.6938 & 0.6806 & 0.6500 & 0.6501 & 0.6487 & 1.2000 & 1.1880 & 1.1573 & 1.1573 & & 682 & 0.5606 & 0.5422 & & 411 \\
\hline 45 & 0.5972 & 0.5815 & 0.5510 & 0.5511 & 0.5497 & 505 & 1.2457 & 1.2149 & 1.2149 & 136 & 543 & 0.5482 & 298 & 299 & 0.5287 \\
\hline 52.5 & 0.5078 & 0.4966 & 0.4662 & 0.4662 & 0.4649 & 859 & 1.2735 & 1.2427 & 1.2428 & 1.2415 & 0.5381 & 0.5311 & 127 & 127 & 117 \\
\hline 60 & 0.4330 & 0.4123 & 0.3819 & 0.3819 & 0.3805 & 1.2990 & 1.3008 & 1.2700 & 1.2701 & 1.2689 & 0.5196 & 0.5140 & 0.4956 & 0.4956 & 0.4947 \\
\hline 67.5 & 0.3784 & 0.3681 & 0.3377 & 0.3377 & 0.3363 & 1.2845 & 1.2733 & 1.2425 & 1.2425 & 1.2414 & 0.4989 & 0.4924 & 0.4741 & 0.4741 & 0.4733 \\
\hline 75 & 0.3472 & 0.3247 & 0.2943 & 0.2944 & 0.2929 & 1.2396 & 1.2450 & 1.2142 & 1.2142 & 1.2132 & 0.4760 & 0.4709 & 0.4526 & 0.4526 & 0.4519 \\
\hline 82.5 & 0.3397 & 0.3288 & 0.2983 & 0.2984 & 0.2969 & 1.1640 & 1.1558 & 1.1250 & 1.1250 & 240 & 511 & 0.4454 & 0.4270 & 271 & 0.4264 \\
\hline 90 & 536 & 0.3336 & 0.3032 & 0.3032 & 0.3017 & 1.0607 & 1.0658 & 1.0351 & 351 & 342 & 243 & 198 & 015 & 15 & 010 \\
\hline 97.5 & 39 & 0.3716 & 0.3411 & 11 & 0 & 48 & 306 & 00 & 000 & 91 & 956 & 07 & 723 & 24 & 720 \\
\hline 105 & 0.4239 & 0.4099 & 0.3795 & 0.3795 & 0.3780 & 0.7936 & 0.7951 & 0.7645 & 0.7645 & 0.7636 & 653 & 0.3615 & 0.3432 & 432 & 0.3430 \\
\hline 112.5 & 0.4655 & 0.4517 & 0.4212 & 0.4213 & 0.4198 & 0.6457 & 0.6457 & 0.6152 & 0.6152 & 0.6144 & 0.3333 & 0.3292 & 0.3109 & 0.3110 & 0.3108 \\
\hline 120 & 0.5000 & 0.4933 & 0.4629 & 0.4629 & 0.4614 & 0.5000 & 0.4965 & 0.4660 & 0.4660 & 0.4653 & 0.3000 & 0.2970 & 0.2787 & 0.2787 & 0.2786 \\
\hline 127.5 & 0.5197 & 0.5058 & 0.4753 & 0.4753 & 0.4739 & 0.3649 & 0.3680 & 0.3375 & 0.3375 & 0.3368 & 0.2654 & 0.2621 & 0.2438 & 0.2439 & 0.2439 \\
\hline 135 & 0.5180 & 0.5175 & 0.4870 & 0.4871 & 0.4857 & 0.2474 & 0.2401 & 0.2097 & 0.2097 & 0.2090 & 0.2296 & 0.2273 & 0.2090 & 0.2091 & 0.2092 \\
\hline 142.5 & 0.4905 & 0.4783 & 0.4479 & 0.4479 & 0.4466 & 0.1523 & 0.1567 & 0.1263 & 0.1263 & 0.1256 & 0.1929 & 0.1905 & 0.1722 & 0.1723 & 0.1725 \\
\hline 150 & 0.4356 & 0.4383 & 0.4078 & 0.4078 & 0.4066 & 0.0820 & 0.0741 & 0.0437 & 0.0437 & 0.0431 & 0.1553 & 0.1537 & 0.1355 & 0.1355 & 0.1358 \\
\hline 157.5 & 0.3542 & 0.3457 & 0.3153 & 0.3153 & 0.3142 & 0.0360 & 0.0396 & 0.0093 & 0.0092 & 0.0086 & 0.1171 & 0.1156 & 0.0974 & 0.0974 & 0.0977 \\
\hline 165 & 0.2501 & 0.2524 & 0.2220 & 0.2220 & 0.2210 & 0.0110 & 0.0058 & -0.0245 & -0.0245 & -0.0251 & 0.0783 & 0.0775 & 0.0593 & 0.0593 & 0.0597 \\
\hline 172.5 & 0.1294 & 0.1262 & 0.0958 & 0.0958 & 0.0949 & 0.0014 & 0.0028 & -0.0275 & -0.0276 & -0.0282 & 0.0392 & 0.0387 & 0.0205 & 0.0205 & 0.0209 \\
\hline 180 & 0.0000 & 0.0009 & -0.0295 & -0.0295 & -0.0303 & 0.0000 & -0.0017 & -0.0320 & -0.0320 & -0.0326 & 0.0000 & -0.0002 & -0.0184 & -0.0184 & -0.0180 \\
\hline
\end{tabular}


Table 5.13 Stress State along the circular path close to the front varied along thickness, $a / W=0.6, t / W=4.0, r / a=0.0078$, Uniform, SECP

\begin{tabular}{|c|c|c|c|c|c|c|c|c|c|c|c|c|c|c|c|}
\hline \multirow{2}{*}{$\theta\left({ }^{\circ}\right)$} & \multicolumn{5}{|c|}{$f_{11}(\theta)$} & \multicolumn{5}{|c|}{$f_{22}(\theta)$} & \multicolumn{5}{|c|}{$f_{33}(\theta)$} \\
\hline & Eq.(5.2) & $z / t=0$ & 0.1161 & 0.2637 & 0.4182 & q. $(5.2)$ & $z / t=0$ & 0.1161 & 0.2637 & 0.4182 & $9 .(5.2)$ & $z / t=0$ & 0.1161 & 0.2637 & 0.4182 \\
\hline 0 & 0900 & 2974 & - & .9664 & 64 & - & 009 & & 488 & 488 & 000 & 0.5931 & 745 & 746 & 742 \\
\hline 7.5 & 9851 & 9678 & 0 & .9368 & 68 & 06 & 229 & 99 & 700 & 699 & 987 & 0.5906 & 720 & 720 & 717 \\
\hline 15 & 419 & 9374 & 64 & 65 & 65 & 110 & 1.0764 & 18 & 19 & 919 & 949 & 881 & 695 & 695 & 692 \\
\hline 22.5 & 745 & 0.8587 & 0.8278 & 8 & 0.8278 & 1.0871 & 1.1304 & 1.0453 & 154 & 1.0453 & 385 & 805 & & 620 & 616 \\
\hline 30 & 891 & 0.7796 & 0.7487 & 0.7488 & 0.7488 & 427 & 1.1880 & 0993 & 0994 & 993 & 796 & 0.5730 & 544 & 5544 & 5541 \\
\hline 37.5 & & 5805 & 0 & 7 & 97 & 00 & 457 & 69 & 70 & 69 & 82 & 306 & 420 & 20 & 417 \\
\hline 45 & & 10 & 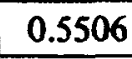 & & & 05 & 35 & & & & & & & & \\
\hline 52.5 & & & & & & & & & & & & & & & \\
\hline 60 & & 3 & & & & & 33 & & & & 96 & 39 & & 154 & \\
\hline 67.5 & 84 & 3681 & 0 & 4 & & & 30 & & 2 & 21 & 89 & 24 & 738 & 1739 & 736 \\
\hline 75 & 3472 & 0.3247 & 0.2939 & 0.2940 & 0.2940 & 396 & 1.1558 & 1.2138 & 1.2139 & 138 & 4760 & 0.4709 & 523 & 0.4524 & 0.4521 \\
\hline 82.5 & 397 & 0.3288 & 0.2980 & 0.2980 & 0.2980 & 640 & 1.0658 & 1.1247 & 247 & 246 & 511 & 0.4454 & 268 & 268 & 266 \\
\hline 90 & 536 & .3336 & 0.3028 & 029 & 0.3028 & 607 & 0.9306 & 47 & 1.0348 & 47 & 243 & 198 & 012 & 013 & 010 \\
\hline 97.5 & 839 & 0.3715 & 0.3407 & 408 & 0.3408 & 348 & 0.7951 & 0.8996 & 0.8997 & 996 & 956 & 906 & 721 & 721 & 719 \\
\hline 105 & & 0.4099 & 0.3 & 92 & 91 & & 157 & 41 & 42 & 41 & 553 & 15 & 29 & 30 & 27 \\
\hline 112.5 & 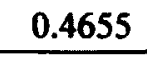 & 0.4517 & 0.4208 & & & 0.6457 & 0.4965 & 0.6148 & 0.6149 & 0.6148 & 0.3333 & 0.3292 & 0.3107 & 0.3107 & 0.3105 \\
\hline 120 & 5000 & 0.4933 & 0.4625 & 0.4625 & & 0.5000 & 0.3680 & & & & 3000 & 0.2969 & & & \\
\hline 127.5 & 197 & 0.5057 & 0.4749 & 0.4750 & & & 0.2401 & & & & 654 & & & 36 & \\
\hline 135 & 180 & 0.5175 & 0.4867 & 0.4867 & 0.4866 & 0.2474 & 0.1567 & 0.2093 & 0.2094 & 0.2093 & 296 & 0.2273 & 0.2088 & 088 & 0.2086 \\
\hline 142.5 & 0.4905 & 0.4783 & 0.4475 & 0.4475 & 0.4474 & 0.1523 & 0.0741 & 0.1259 & 0.1260 & 0.1259 & 0.1929 & 0.1905 & .1720 & 0.1720 & 0.1718 \\
\hline 150 & 356 & 0.4383 & 0.4074 & 0.4075 & 0.4074 & 820 & 0.0396 & 0.0434 & 0.0434 & 0.0433 & 1553 & 0.1537 & 352 & 0.1352 & .1350 \\
\hline 157.5 & 0.3542 & 0.3457 & 0.3149 & 0.3150 & 0.3148 & 0.0360 & 0.0058 & 0.0089 & 0.0089 & 0.0089 & 0.1171 & 0.1156 & 0.0971 & 0.0971 & 0.0969 \\
\hline 165 & 0.2501 & 0.2524 & 0.2216 & 0.2217 & 0.2216 & 0.0110 & 0.0028 & -0.0248 & -0.0248 & -0.0249 & 0.0783 & 0.0775 & 0.0590 & 0.0590 & 0.0588 \\
\hline 172.5 & 0.1294 & 0.1262 & 0.0955 & 0.0955 & 0.0954 & 0.0014 & -0.0017 & -0.0279 & -0.0279 & -0.0279 & 0.0392 & 0.0387 & 0.0203 & 0.0203 & 0.0201 \\
\hline 180 & 0.0000 & 0.0008 & -0.0298 & -0.0298 & -0.0299 & 0.0000 & 0.0000 & -0.0324 & -0.0323 & -0.0324 & 0.0000 & -0.0003 & -0.0187 & -0.0187 & -0.0189 \\
\hline
\end{tabular}




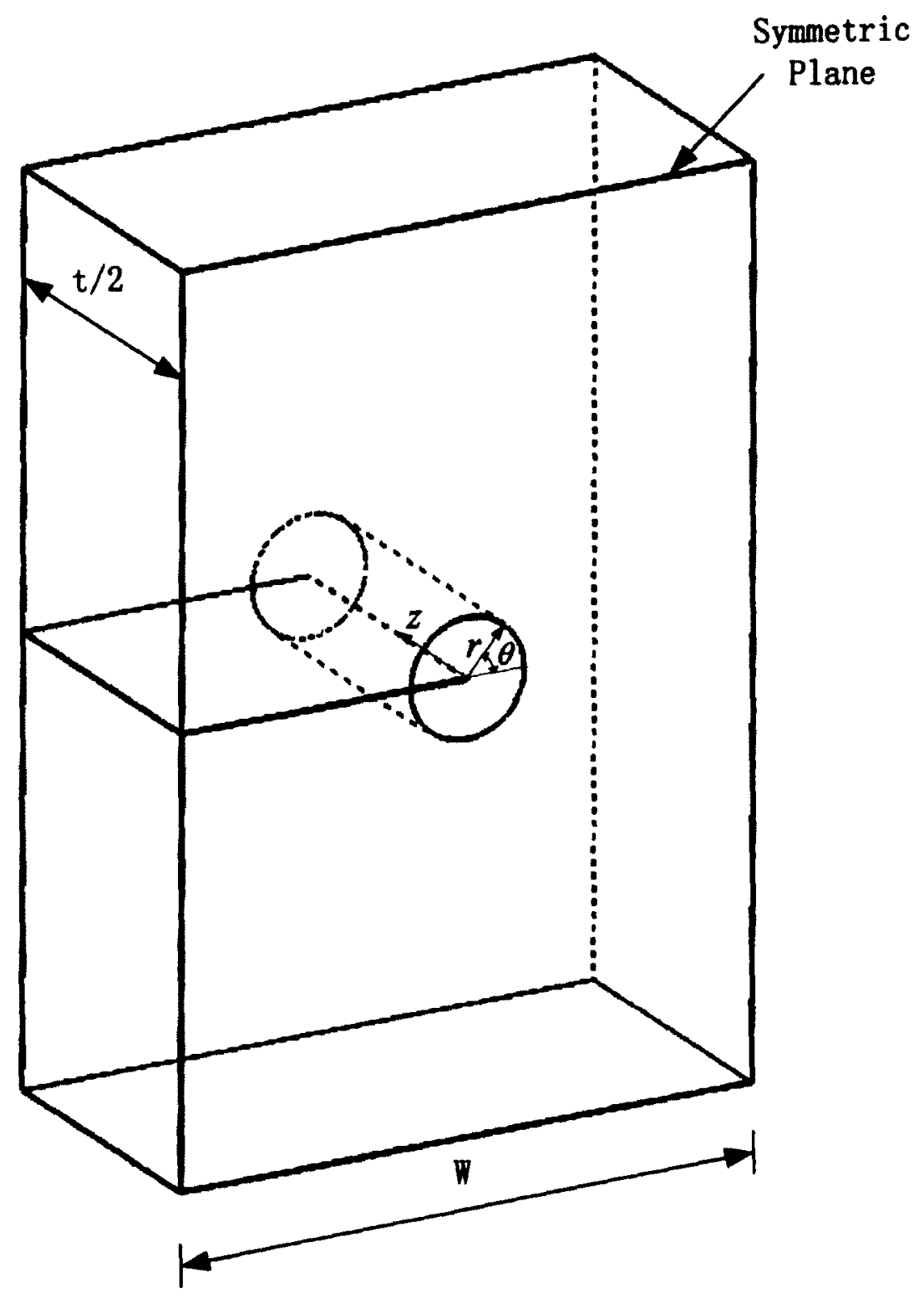

Figure 5.1 Coordinate system for stress state of crack front 


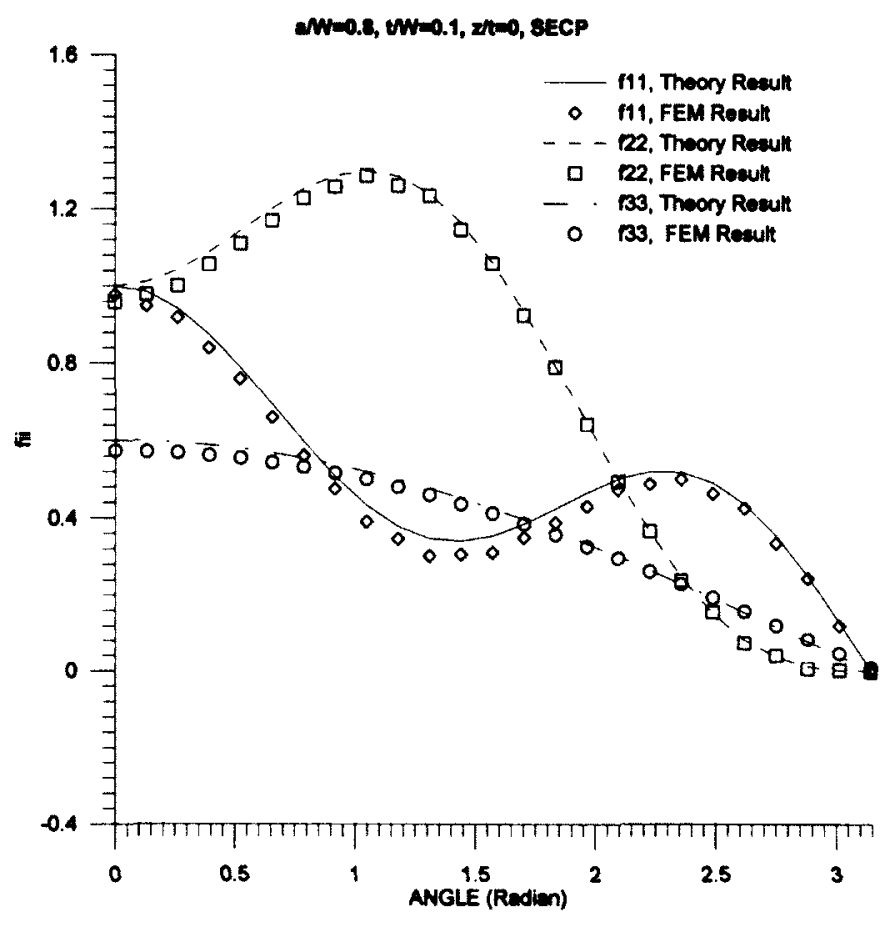

Figure 5.2 Stress State along the circular path close to the front at $z / t=0, a / W=0.8$, $t / W=0.1$

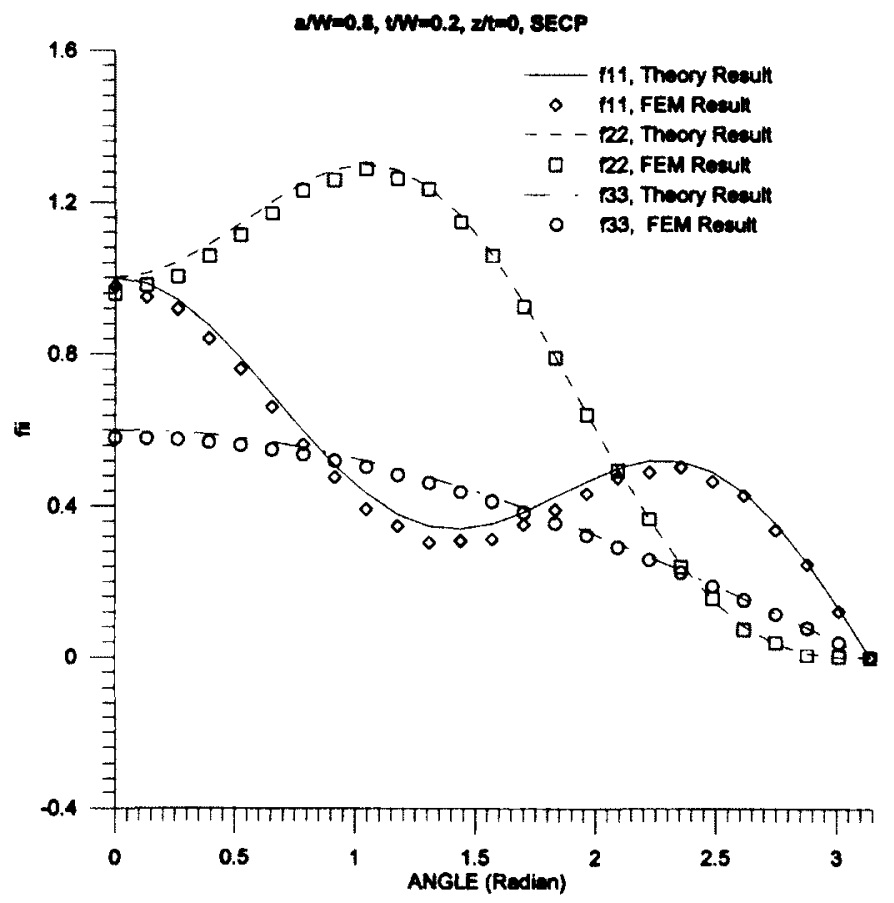

Figure 5.3 Stress State along the circular path close to the front at $z / t=0, a / W=0.8$, $t / W=0.2$ 


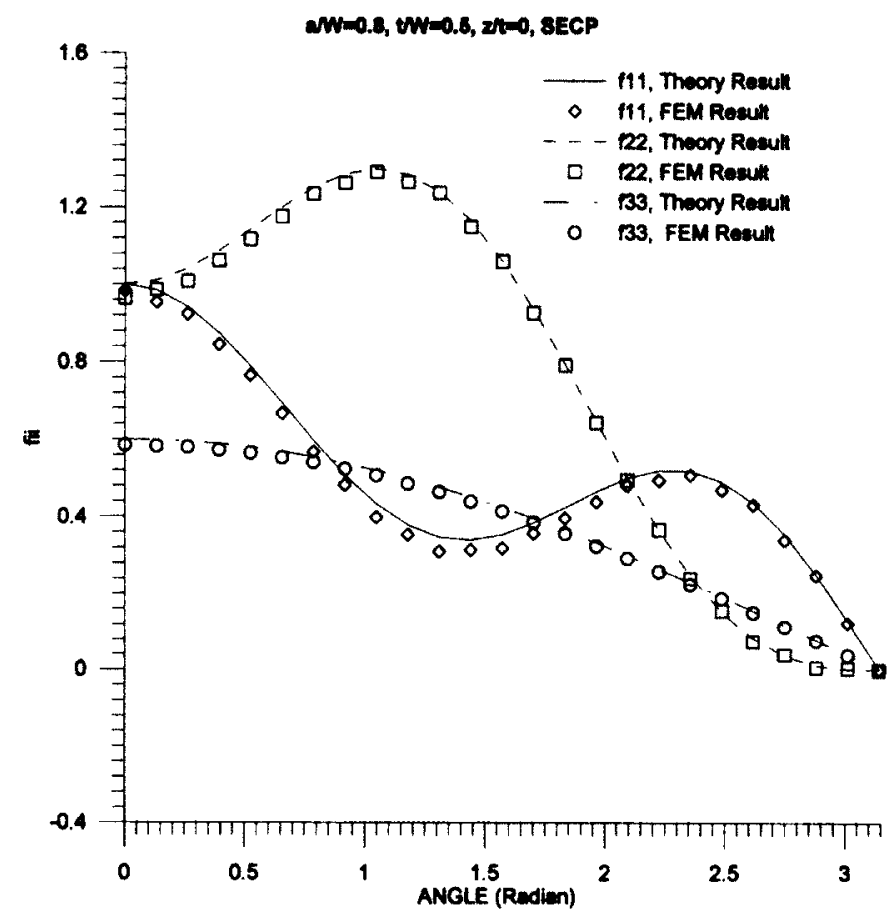

Figure 5.4 Stress State along the circular path close to the front at $z / t=0, a / W=0.8$,

$$
t / W=0.5
$$

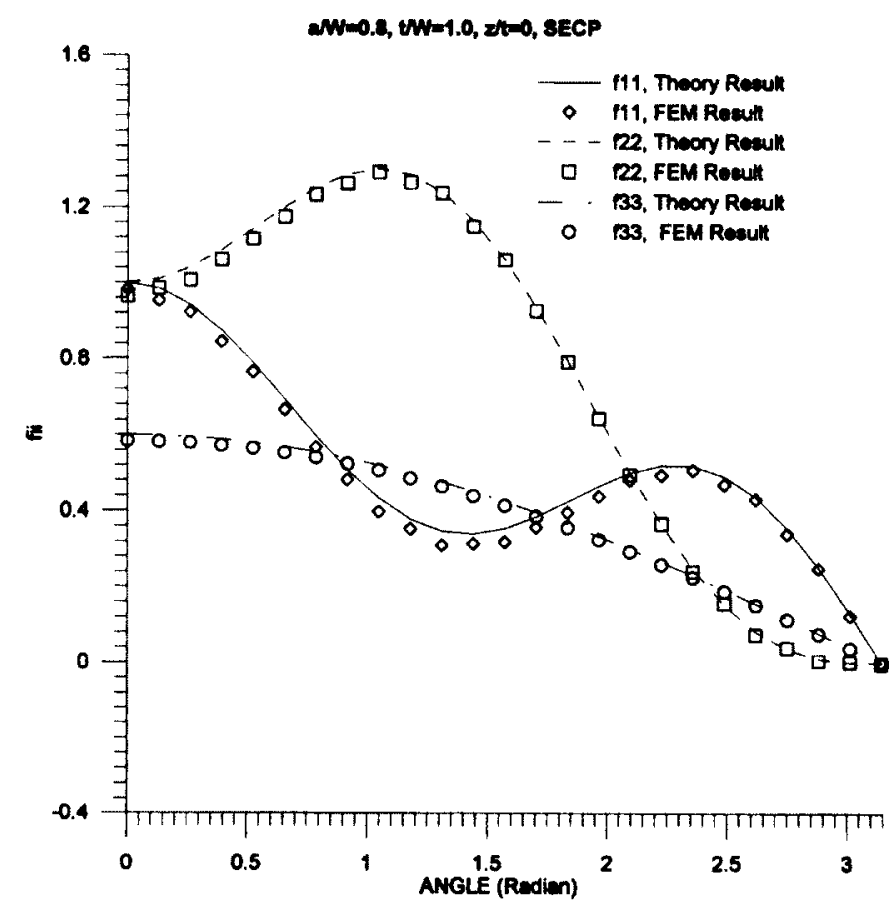

Figure 5.5 Stress State along the circular path close to the front at $z / t=0, a / W=0.8$, $t / W=1.0$ 


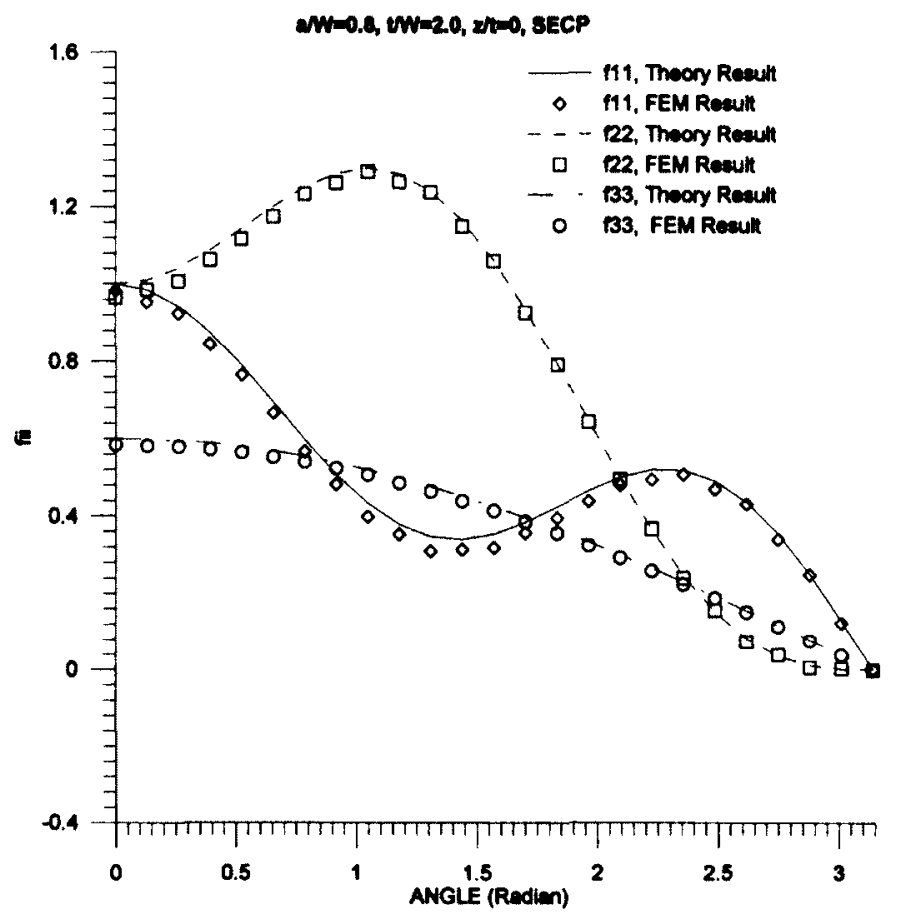

Figure 5.6 Stress State along the circular path close to the front at $z / t=0, a / W=0.8$, $t / W=2.0$

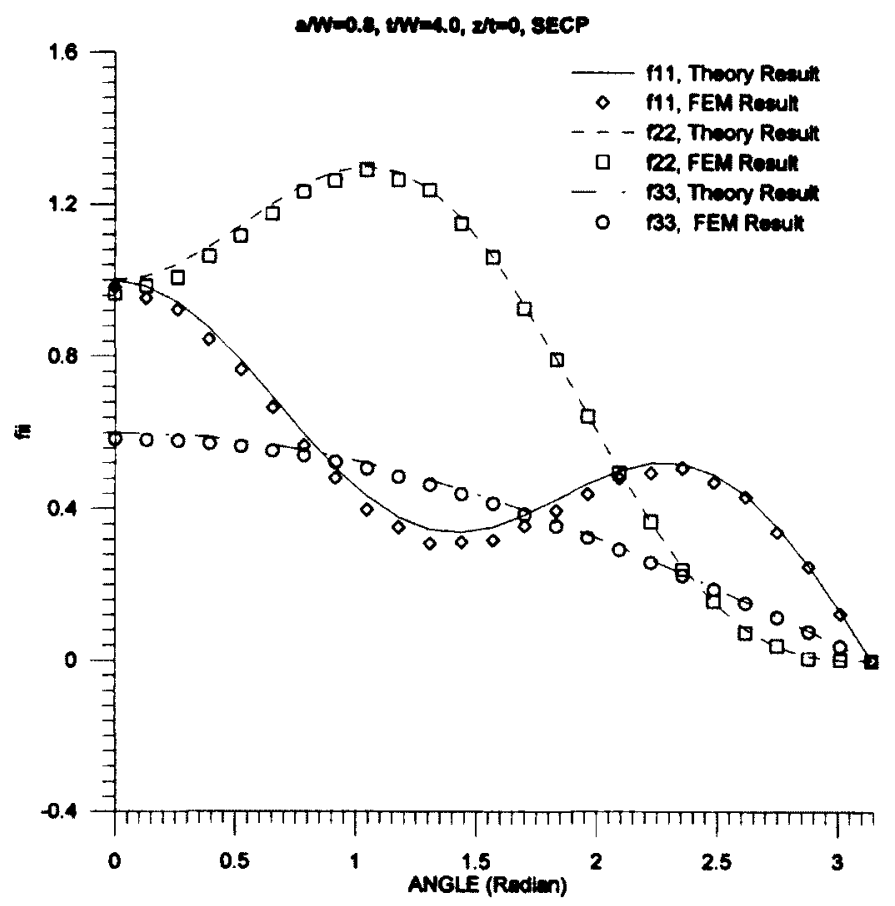

Figure 5.7 Stress State along the circular path close to the front at $z / t=0, a / W=0.8$, $t / W=4.0$ 


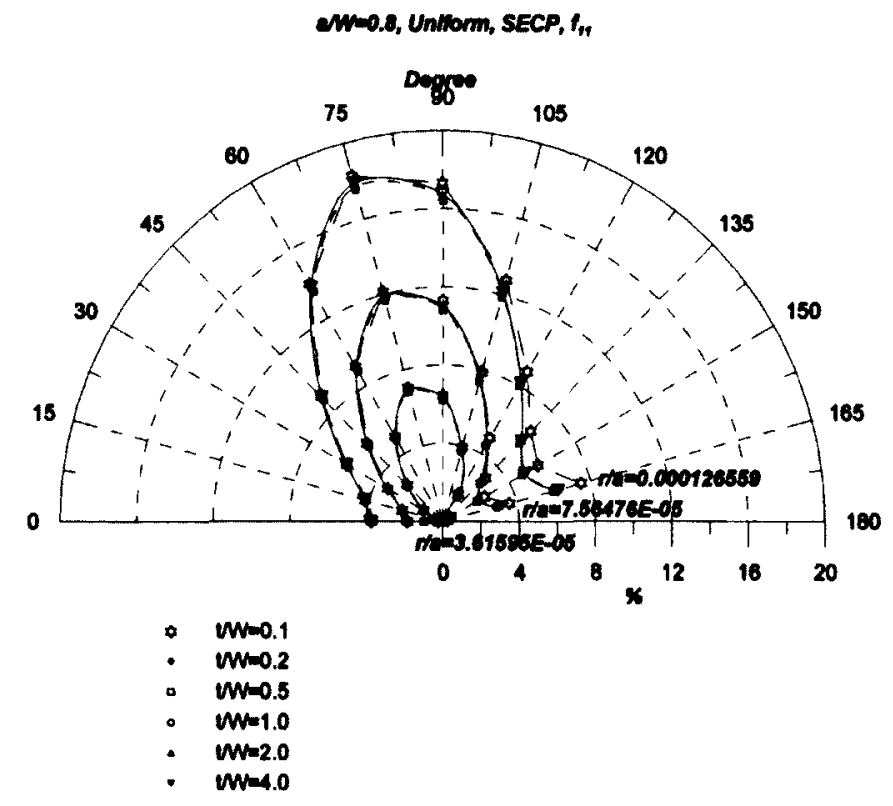

Figure 5.8 Percentage different of $f_{11}(\theta)$ varied by angles at three radiuses location, $a / W=0.8$, Uniform

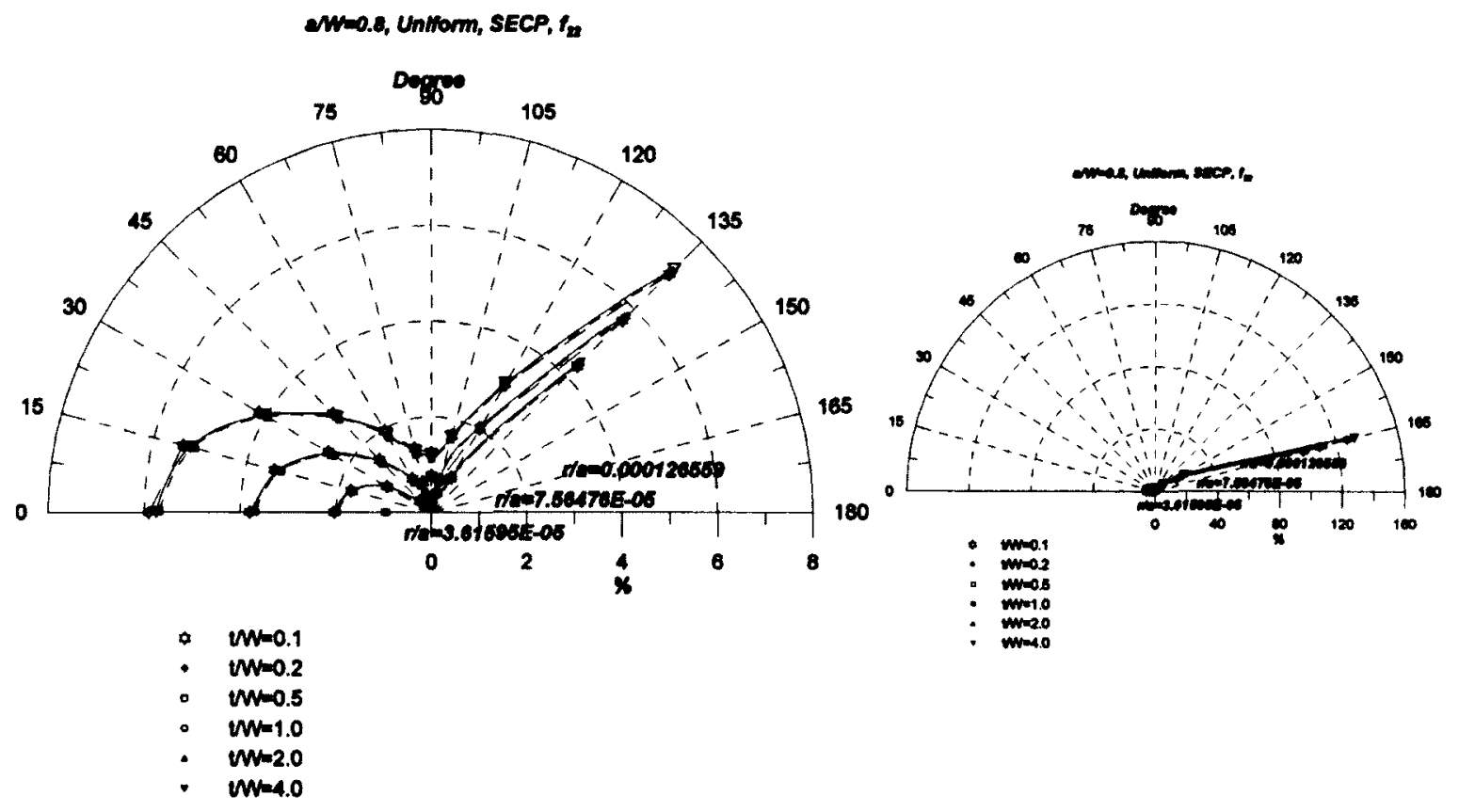

(a)

(b)

Figure 5.9 Percentage different of $f_{22}(\theta)$ varied by angles at three radiuses location, $a / W=0.8$, Uniform: (a) Degree from $0^{\circ}$ to $135^{\circ}$, (b) Degree from $0^{\circ}$ to $165^{\circ}$ 

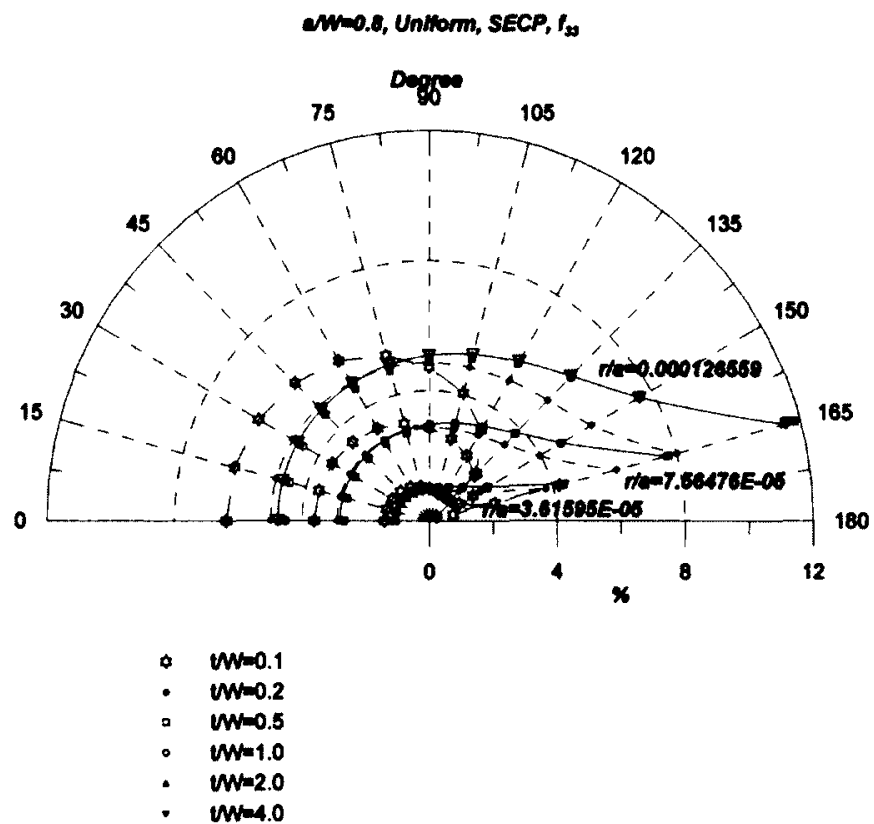

Figure 5.10 Percentage different of $f_{33}(\theta)$ varied by angles at three radiuses location, $a / W=0.8$, Uniform

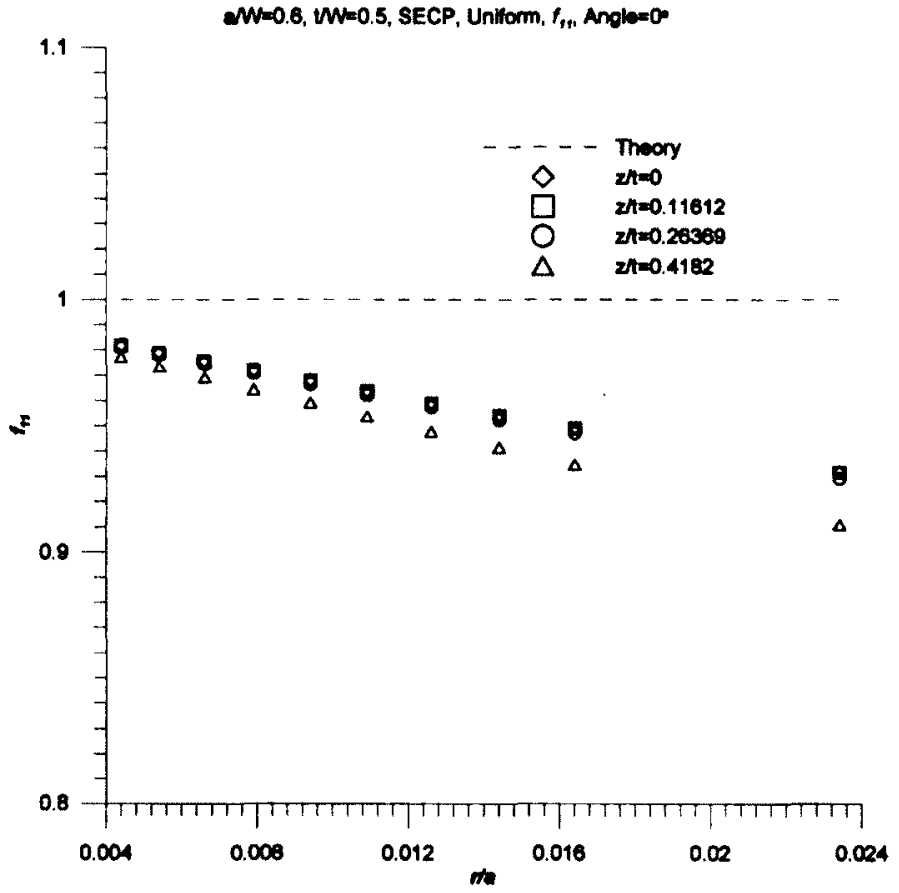

Figure 5.11 Stress State along the circular path close to the front varied by radius at four locations along thickness, $a / W=0.6, t / W=0.5, f_{1 /}$, Angle $=0^{\circ}$, Uniform, SECP 


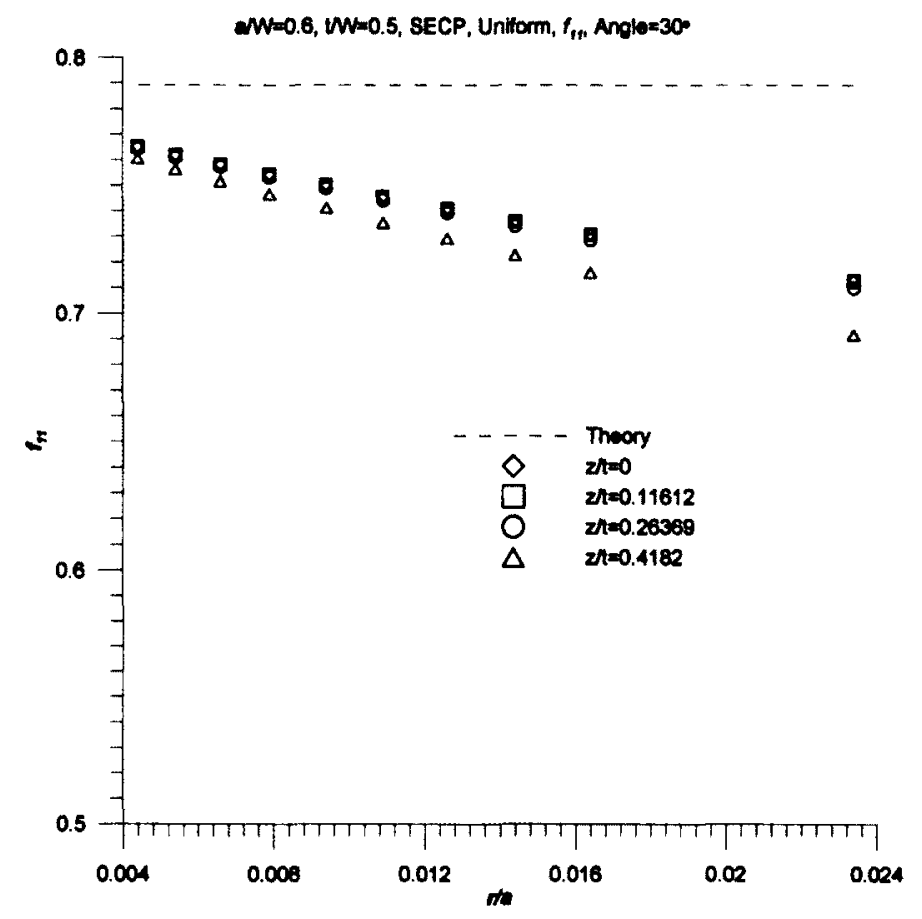

Figure 5.12 Stress State along the circular path close to the front varied by radius at four locations along thickness, $a / W=0.6, t / W=0.5, f_{11}$, Angle $=30^{\circ}$, Uniform, SECP

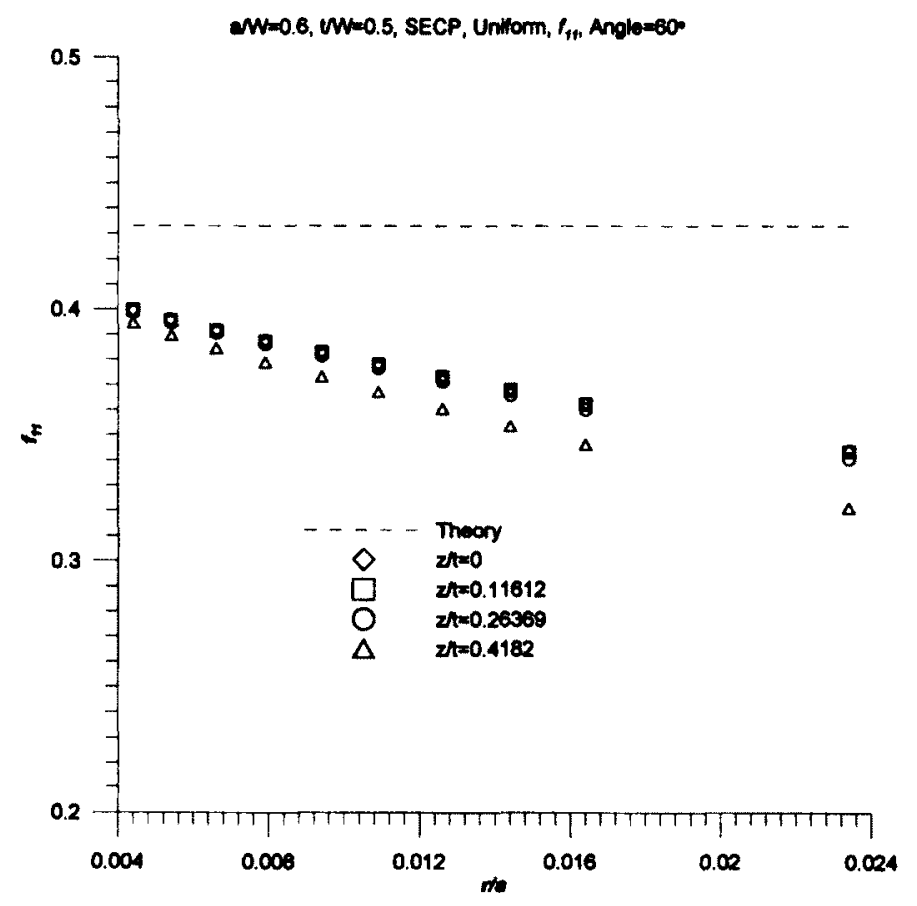

Figure 5.13 Stress State along the circular path close to the front varied by radius at four locations along thickness, $a / W=0.6, t / W=0.5, f_{1 l}$, Angle $=60^{\circ}$, Uniform, SECP 


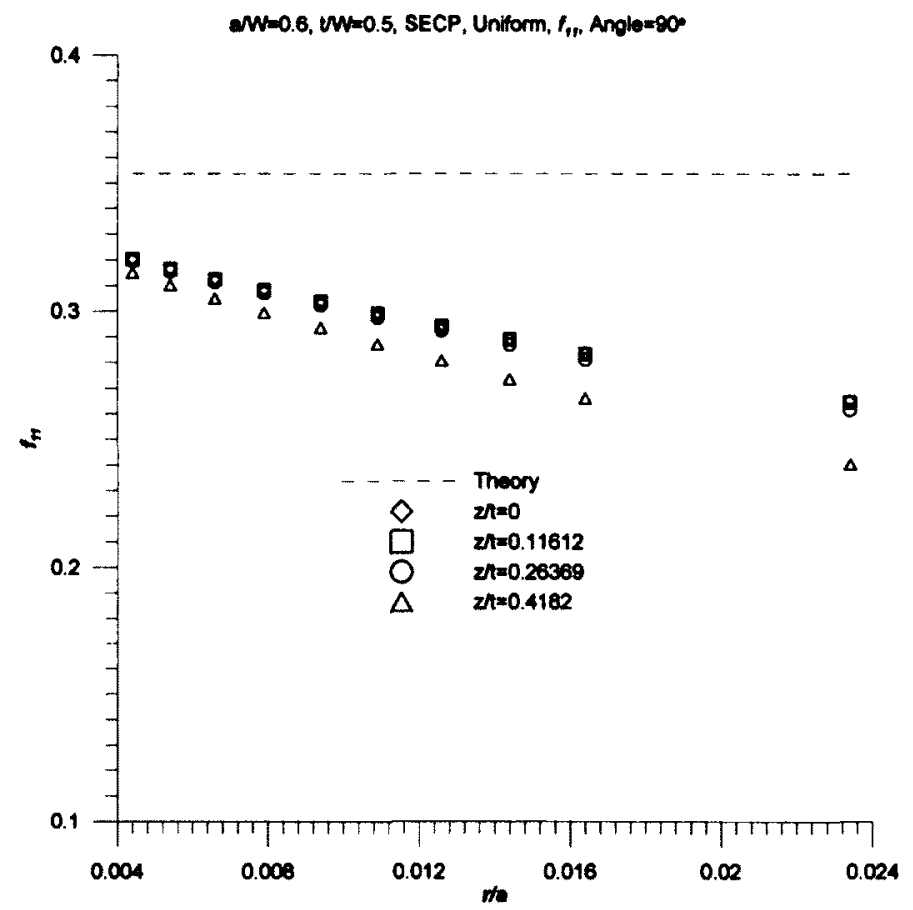

Figure 5.14 Stress State along the circular path close to the front varied by radius at four locations along thickness, $a / W=0.6, t / W=0.5, f_{11}$, Angle $=90^{\circ}$, Uniform, SECP

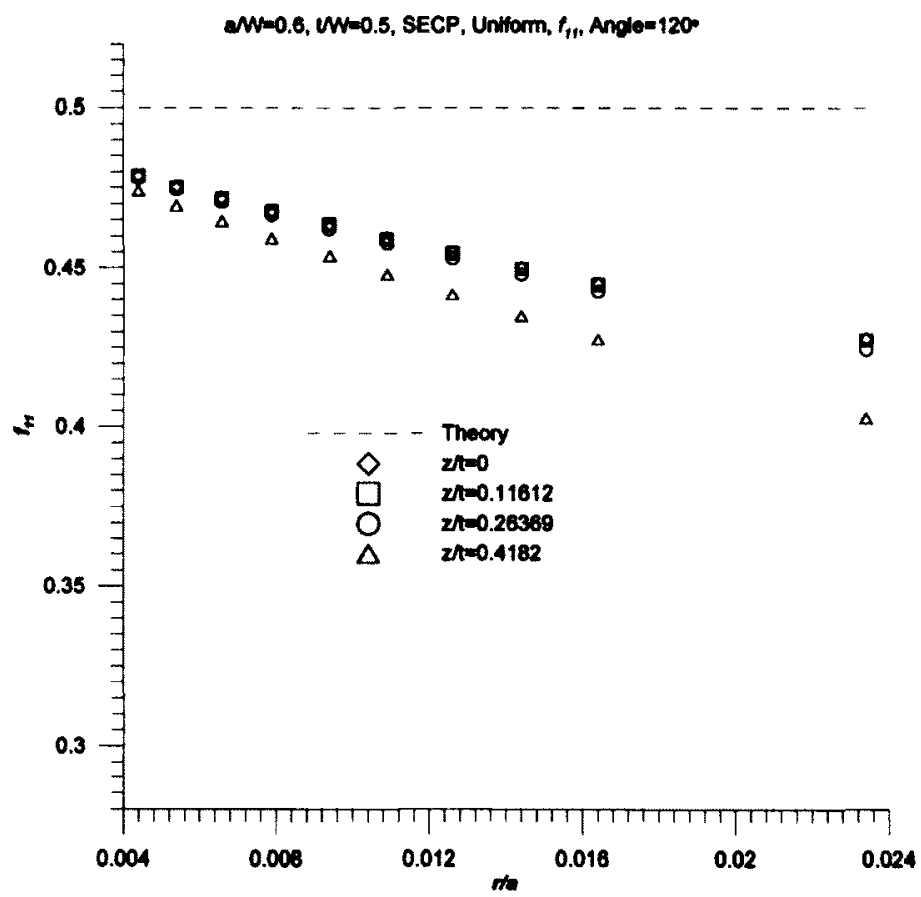

Figure 5.15 Stress State along the circular path close to the front varied by radius at four locations along thickness, $a / W=0.6, t / W=0.5, f_{11}$, Angle $=120^{\circ}$, Uniform, SECP 


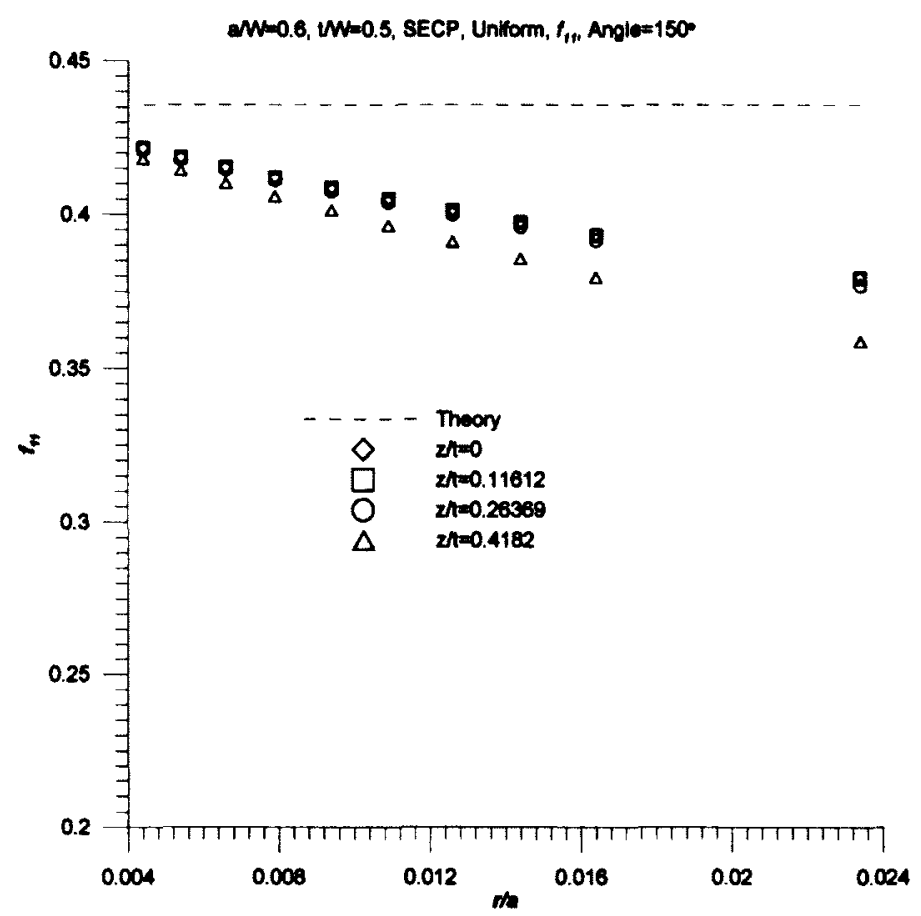

Figure 5.16 Stress State along the circular path close to the front varied by radius at four locations along thickness, $a / W=0.6, t / W=0.5, f_{l l}$, Angle $=150^{\circ}$, Uniform, SECP

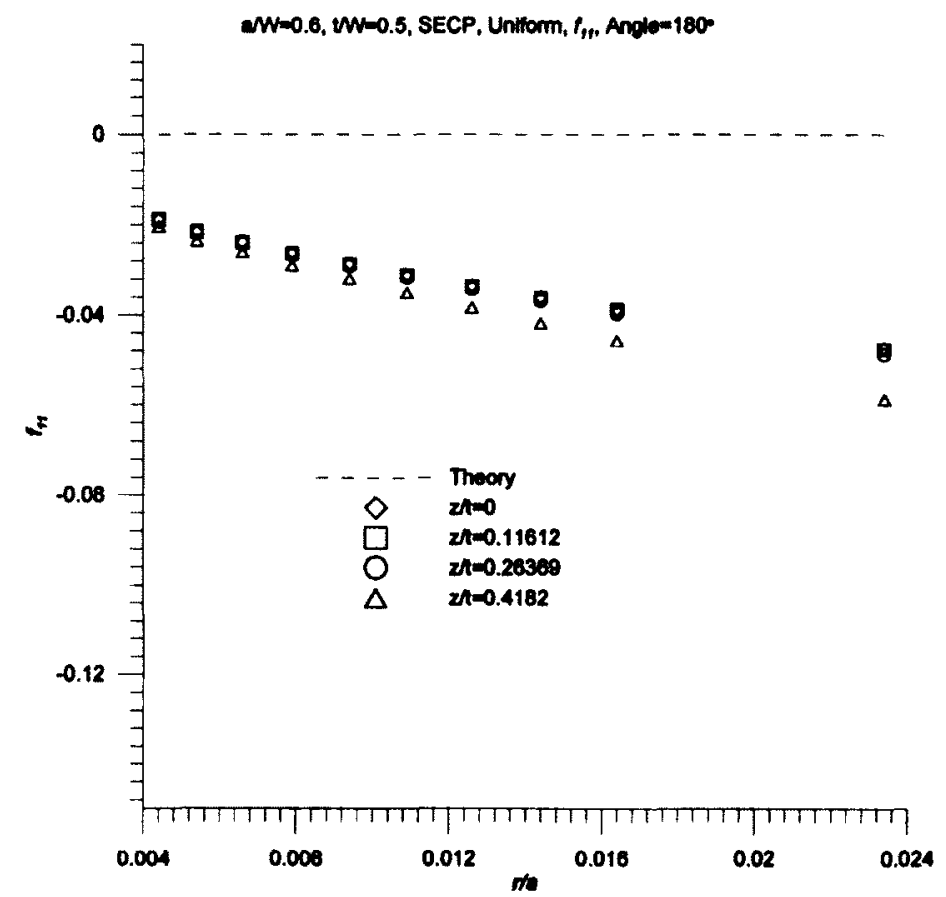

Figure 5.17 Stress State along the circular path close to the front varied by radius at four locations along thickness, $a / W=0.6, t / W=0.5, f_{l l}$, Angle $=180^{\circ}$, Uniform, SECP 


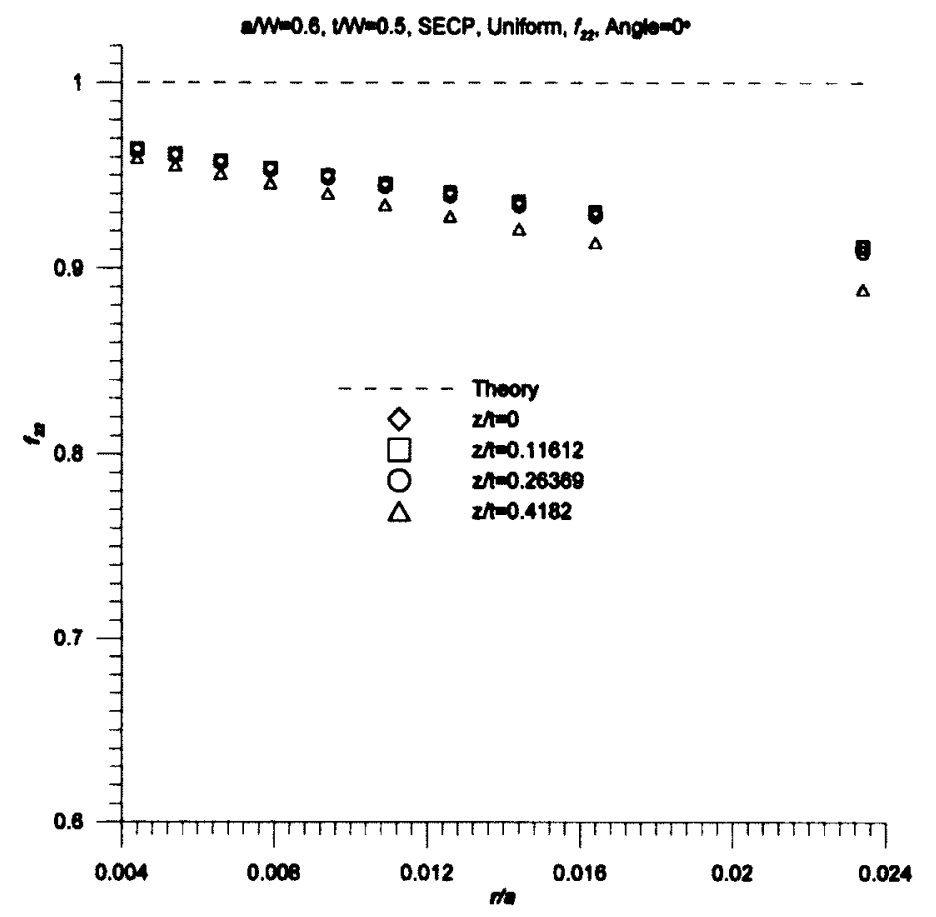

Figure 5.18 Stress State along the circular path close to the front varied by radius at four locations along thickness, $a / W=0.6, t / W=0.5, f_{22}$, Angle $=0^{\circ}$, Uniform, SECP

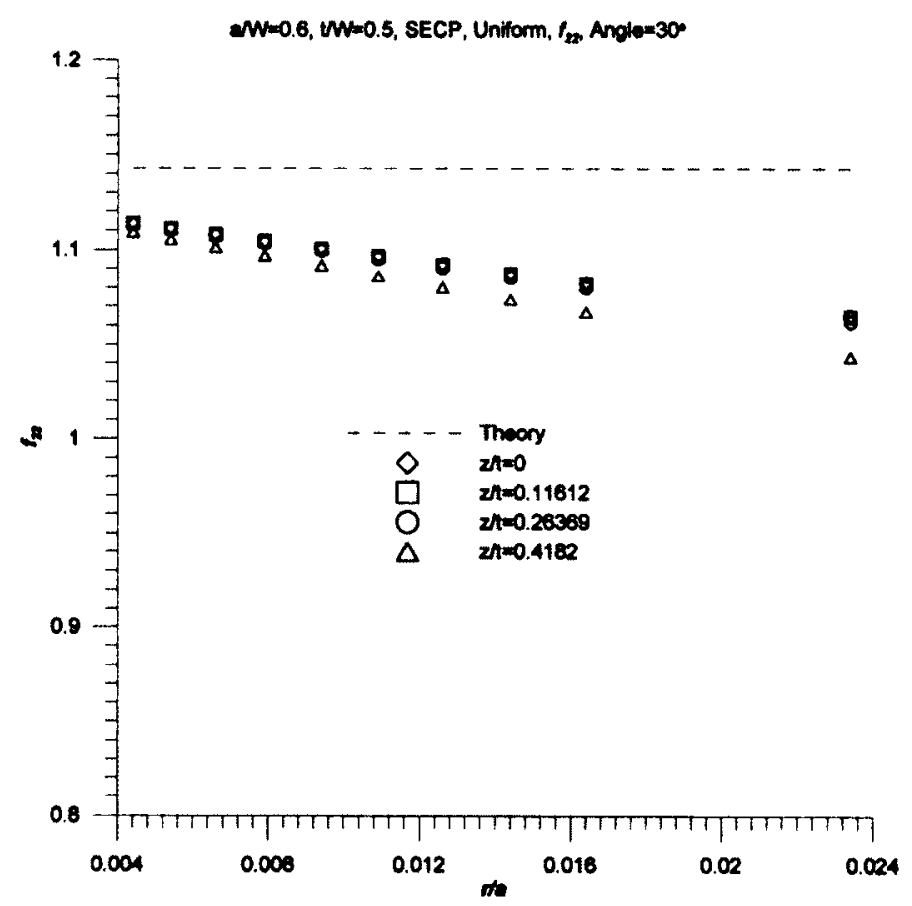

Figure 5.19 Stress State along the circular path close to the front varied by radius at four locations along thickness, $a / W=0.6, t / W=0.5, f_{22}$, Angle $=30^{\circ}$, Uniform, SECP 


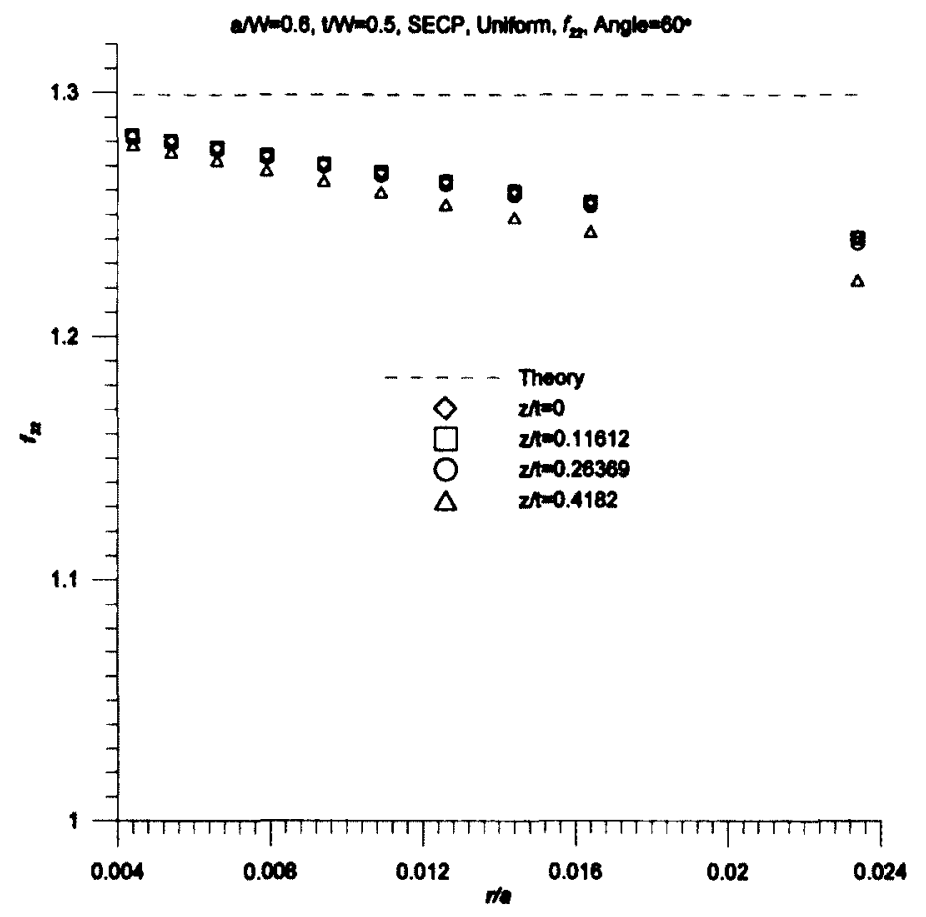

Figure 5.20 Stress State along the circular path close to the front varied by radius at four locations along thickness, $a / W=0.6, t / W=0.5, f_{22}$, Angle $=60^{\circ}$, Uniform, SECP

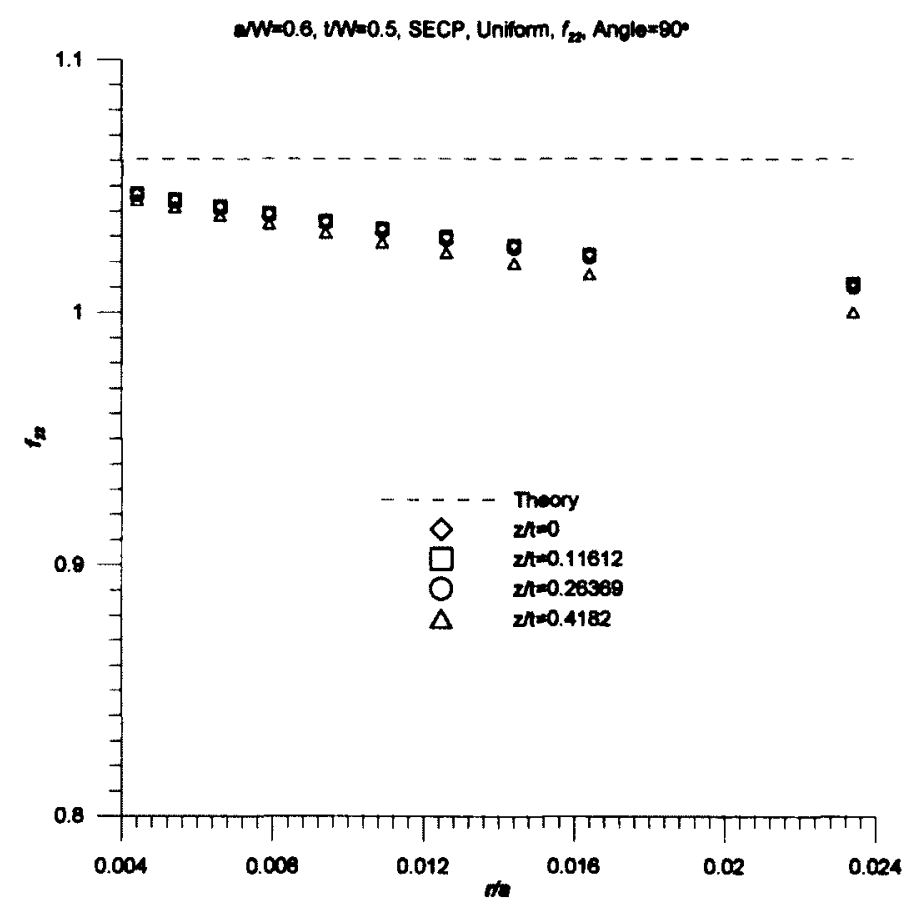

Figure 5.21 Stress State along the circular path close to the front varied by radius at four locations along thickness, $a / W=0.6, t / W=0.5, f_{22}$, Angle $=90^{\circ}$, Uniform, SECP 


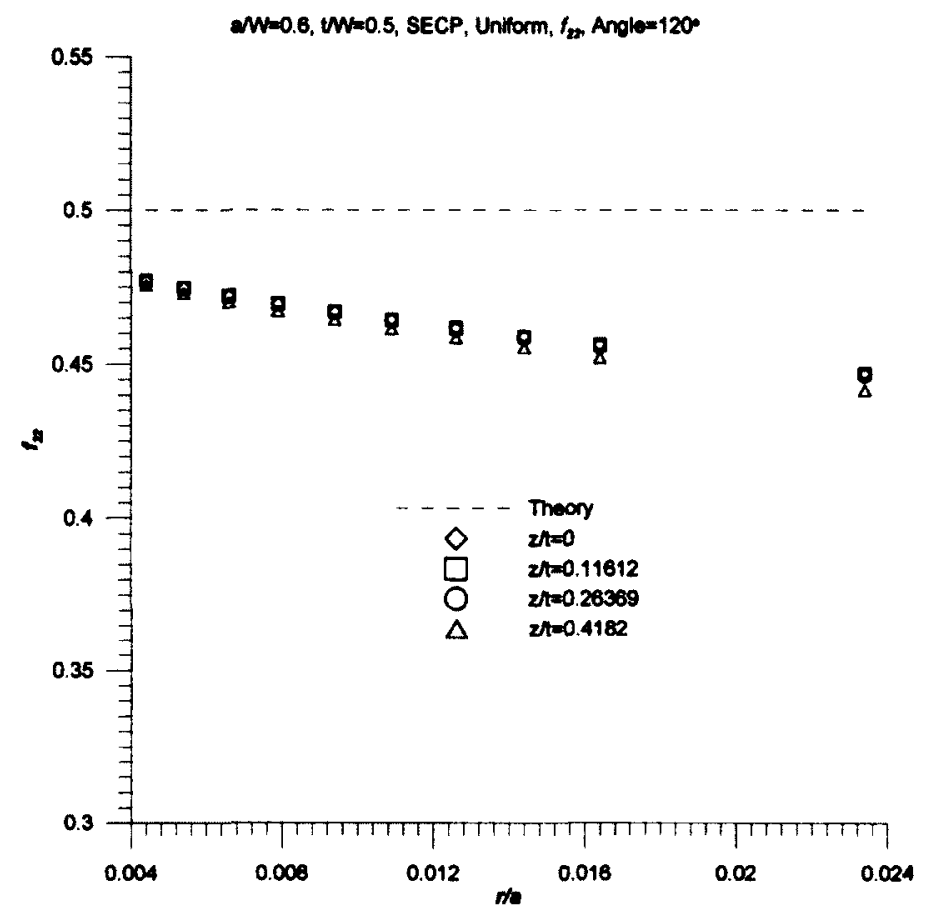

Figure 5.22 Stress State along the circular path close to the front varied by radius at four locations along thickness, $a / W=0.6, t / W=0.5, f_{22}$, Angle $=120^{\circ}$, Uniform, SECP

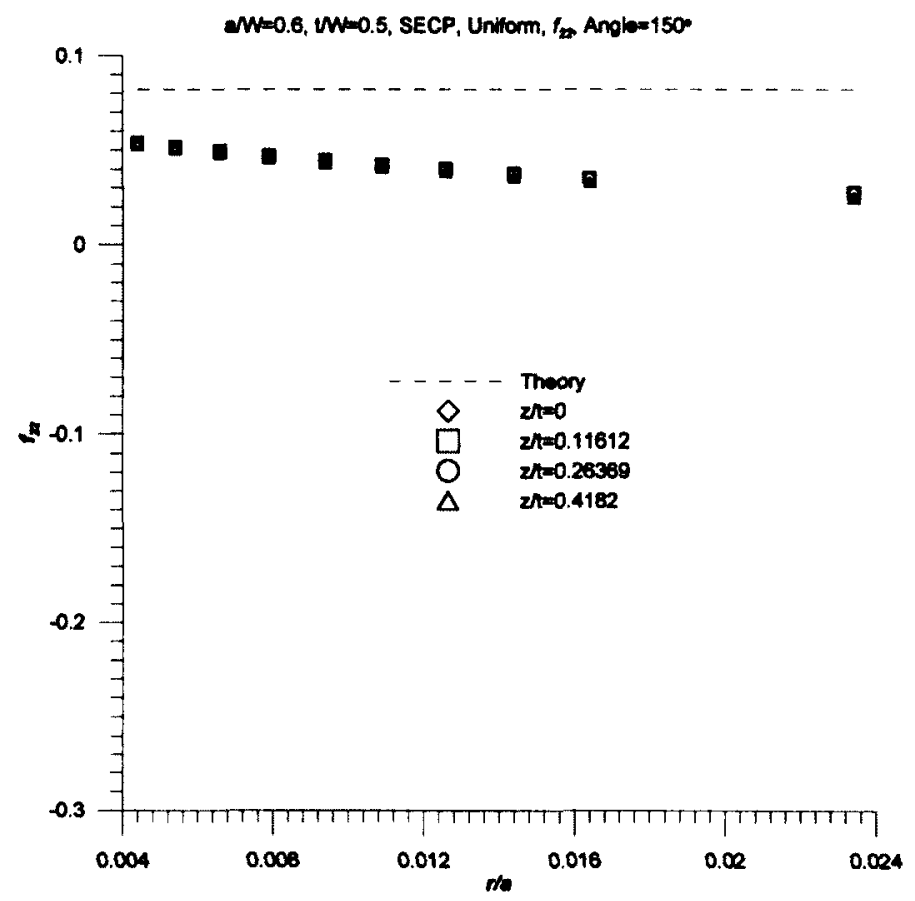

Figure 5.23 Stress State along the circular path close to the front varied by radius at four locations along thickness, $a / W=0.6, t / W=0.5, f_{22}$, Angle $=150^{\circ}$, Uniform, SECP 


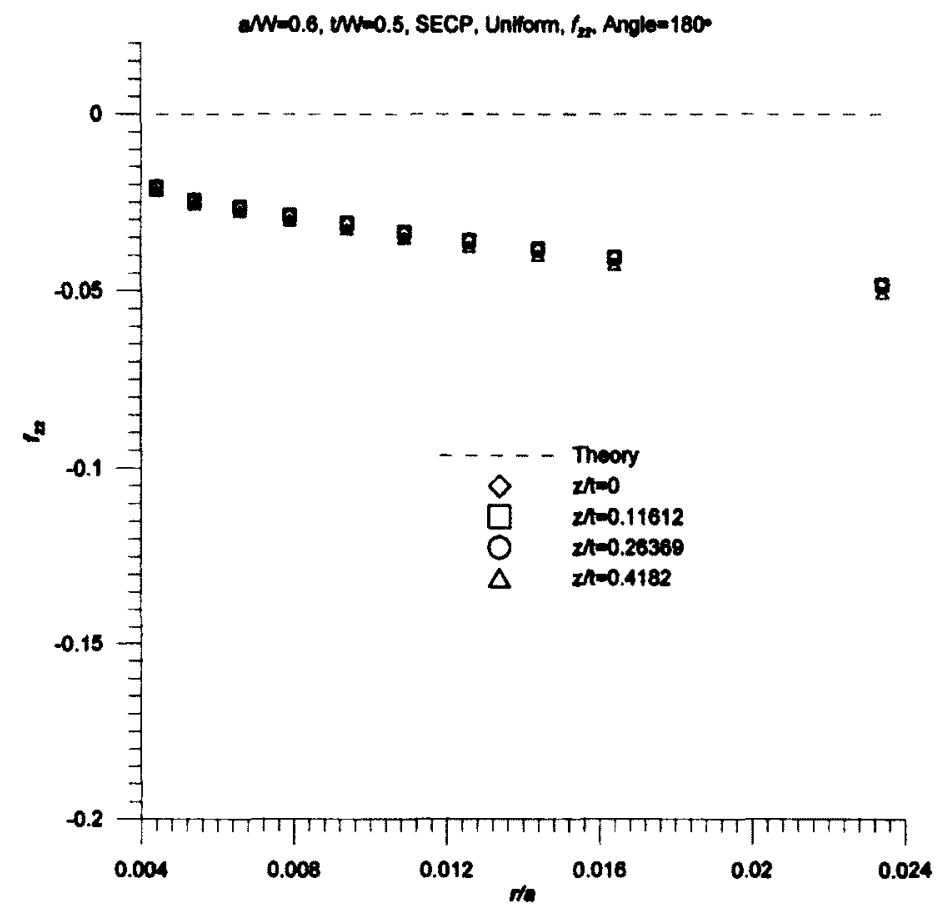

Figure 5.24 Stress State along the circular path close to the front varied by radius at four locations along thickness, $a / W=0.6, t / W=0.5, f_{22}$, Angle $=180^{\circ}$, Uniform, SECP

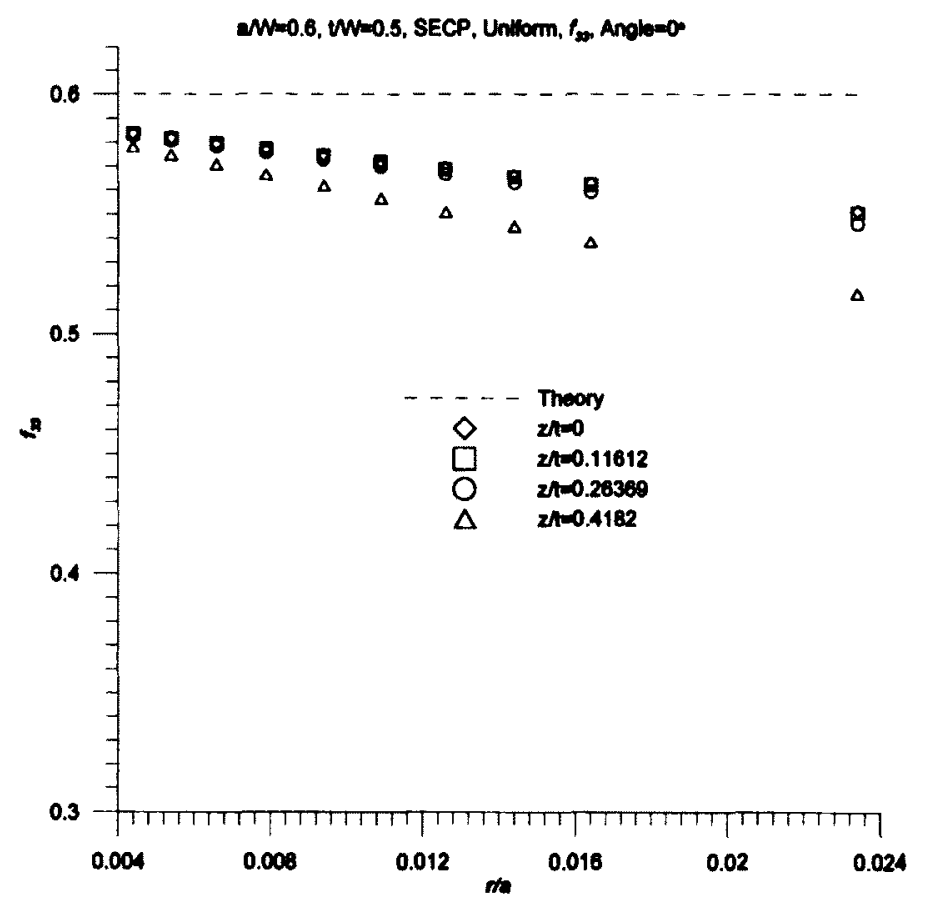

Figure 5.25 Stress State along the circular path close to the front varied by radius at four locations along thickness, $a / W=0.6, t / W=0.5, f_{33}$, Angle $=0^{\circ}$, Uniform, SECP 


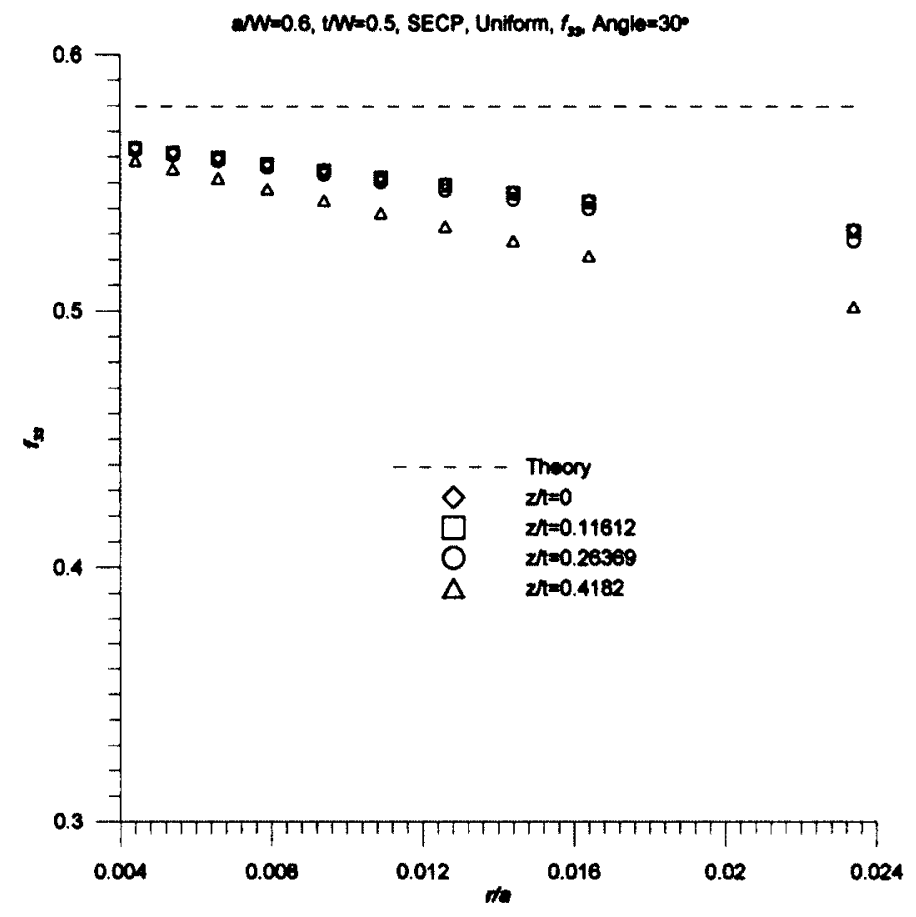

Figure 5.26 Stress State along the circular path close to the front varied by radius at four locations along thickness, $a / W=0.6, t / W=0.5, f_{33}$, Angle $=30^{\circ}$, Uniform, SECP

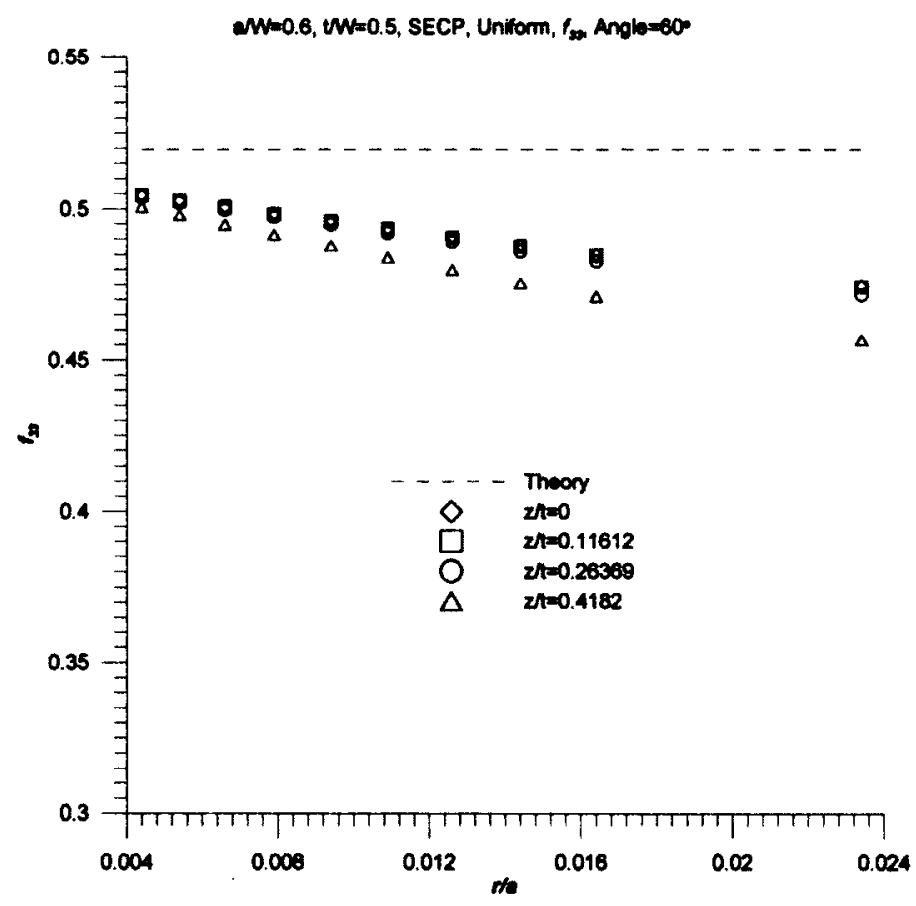

Figure 5.27 Stress State along the circular path close to the front varied by radius at four locations along thickness, $a / W=0.6, t / W=0.5, f_{33}$, Angle $=60^{\circ}$, Uniform, SECP 


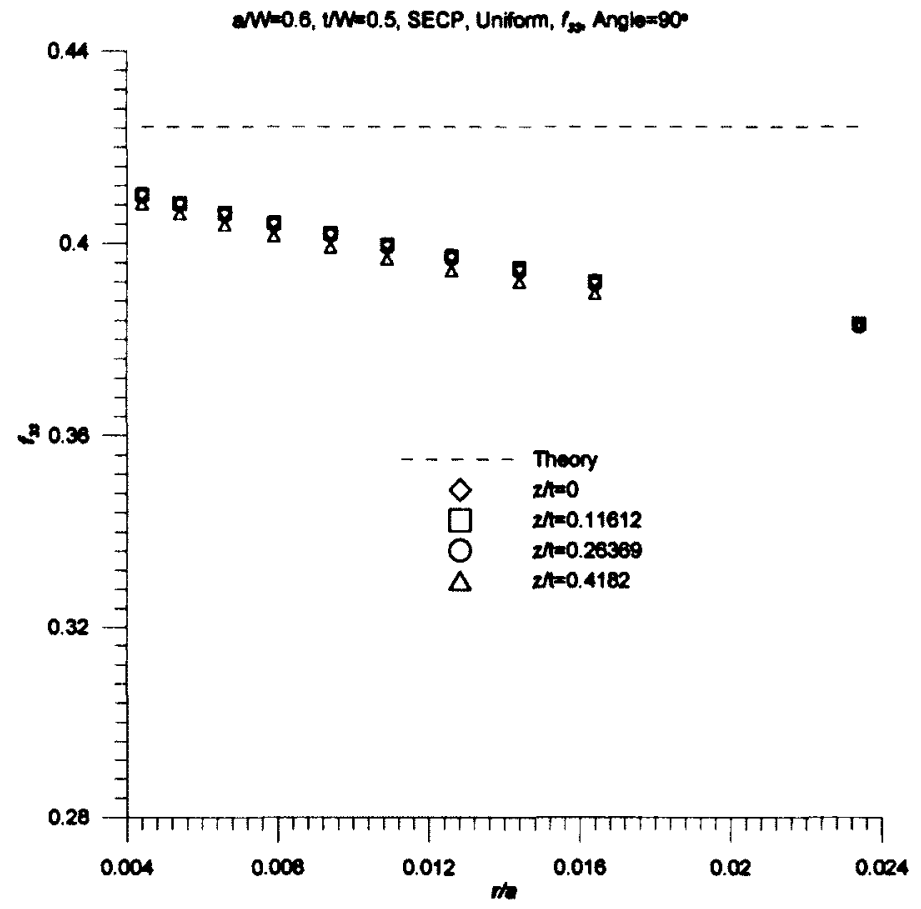

Figure 5.28 Stress State along the circular path close to the front varied by radius at four locations along thickness, $a / W=0.6, t / W=0.5, f_{33}$, Angle $=90^{\circ}$, Uniform, SECP

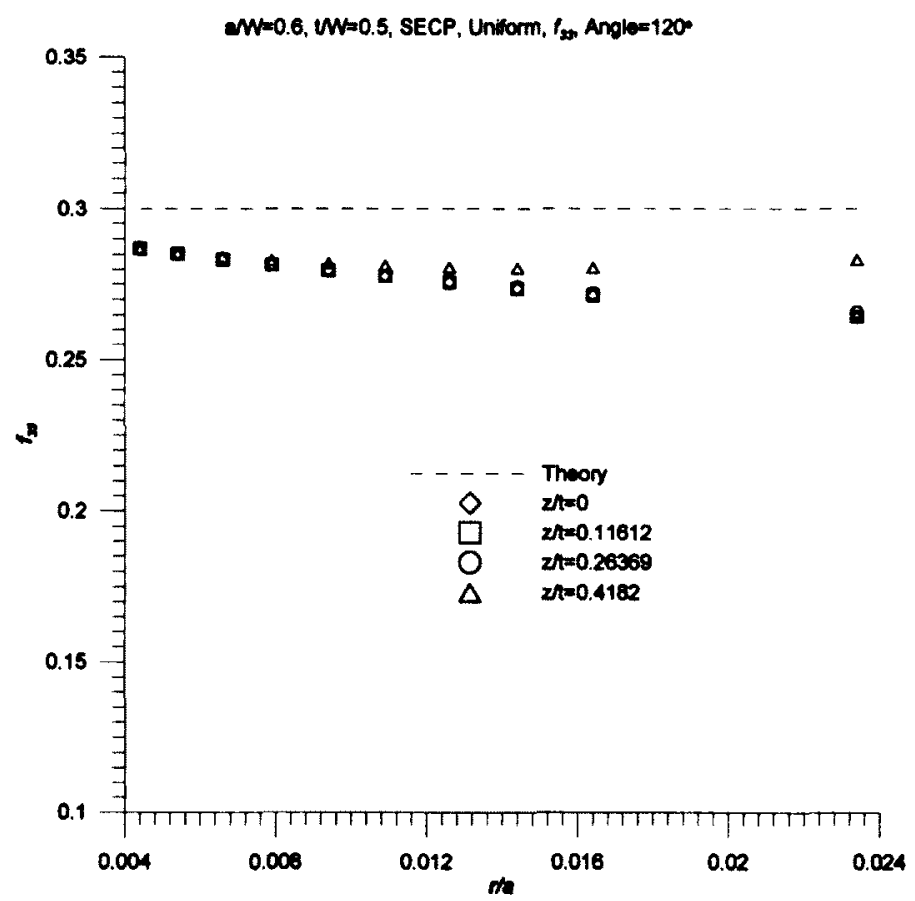

Figure 5.29 Stress State along the circular path close to the front varied by radius at four locations along thickness, $a / W=0.6, t / W=0.5, f_{33}$, Angle $=120^{\circ}$, Uniform, SECP 


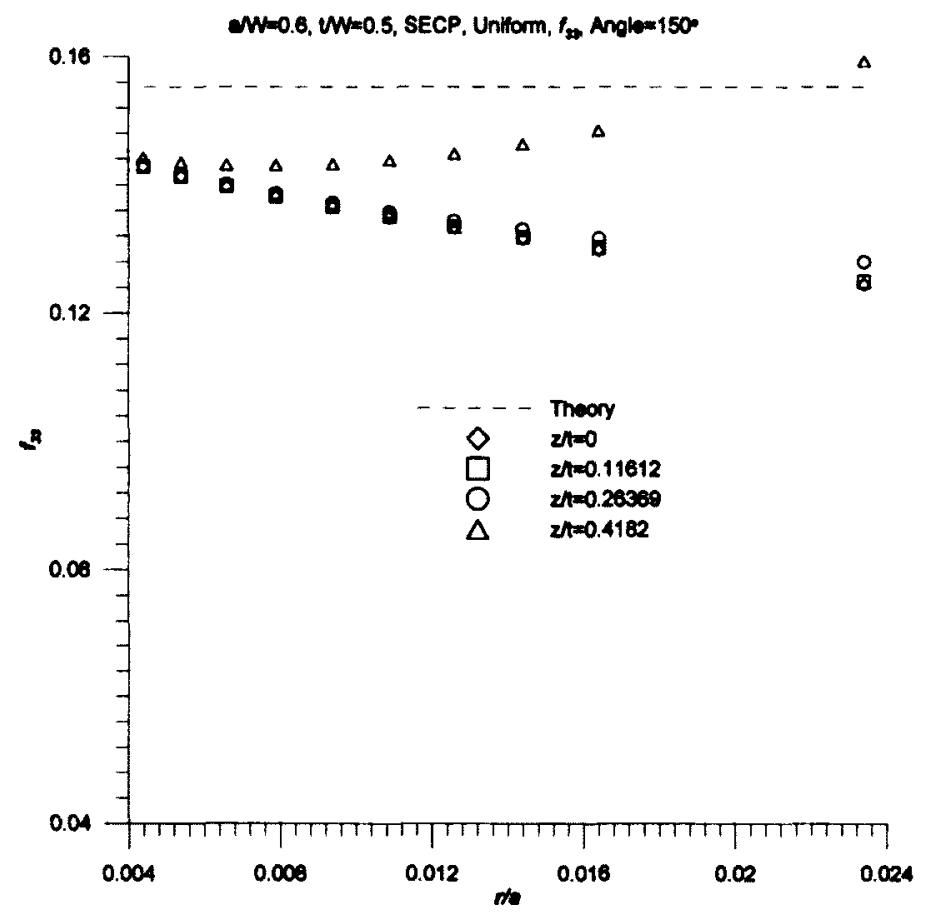

Figure 5.30 Stress State along the circular path close to the front varied by radius at four locations along thickness, $a / W=0.6, t / W=0.5, f_{33}$, Angle $=150^{\circ}$, Uniform, SECP

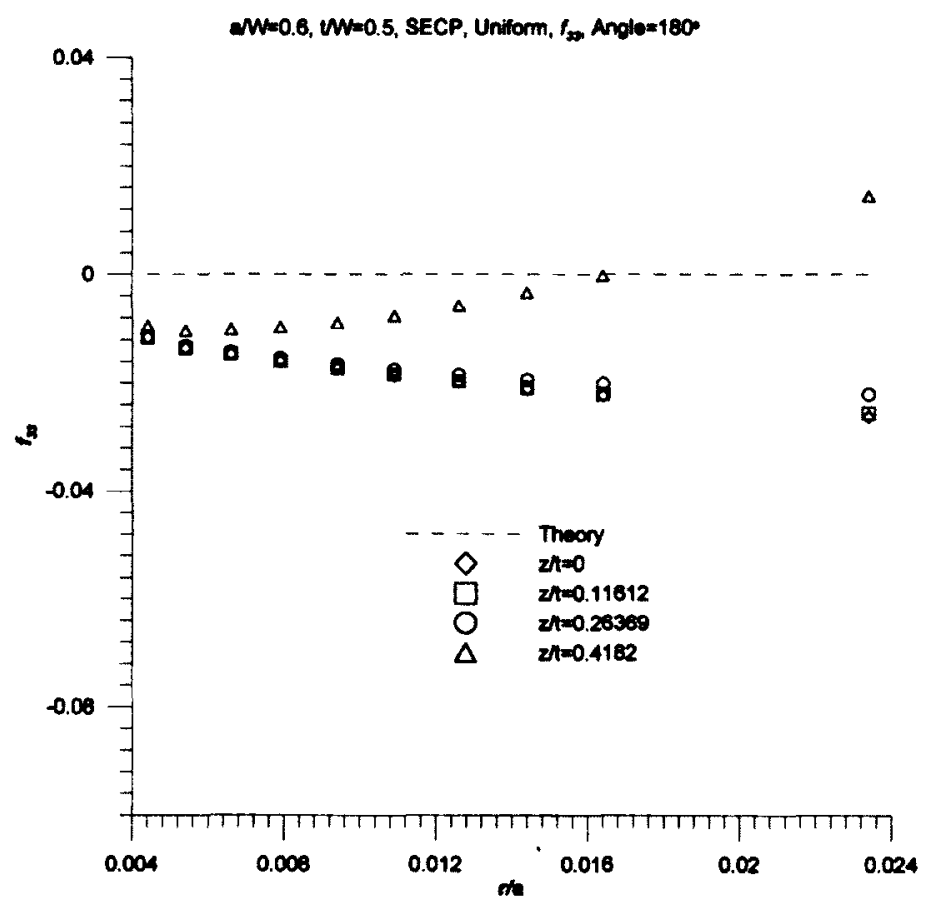

Figure 5.31 Stress State along the circular path close to the front varied by radius at four locations along thickness, $a / W=0.6, t / W=0.5, f_{33}$, Angle $=180^{\circ}$, Uniform, SECP 


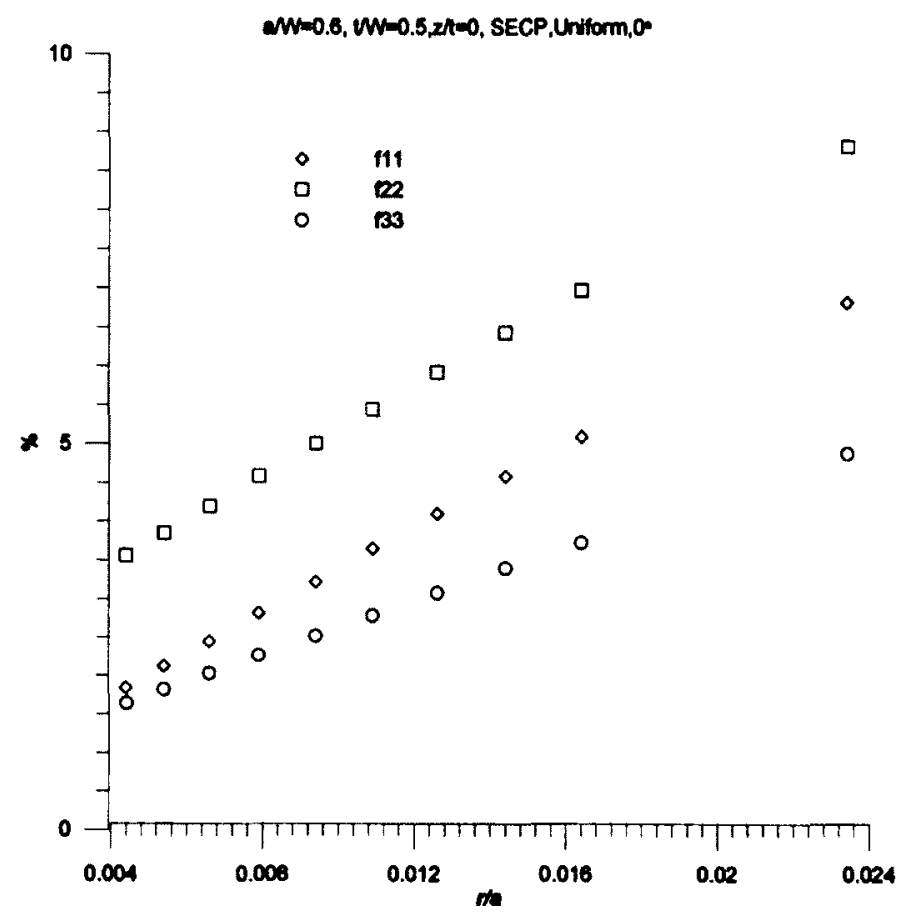

Figure 5.32 Percentage different varied by radius, $a / W=0.6, t / W=0.5$, Uniform, $0^{\circ}$

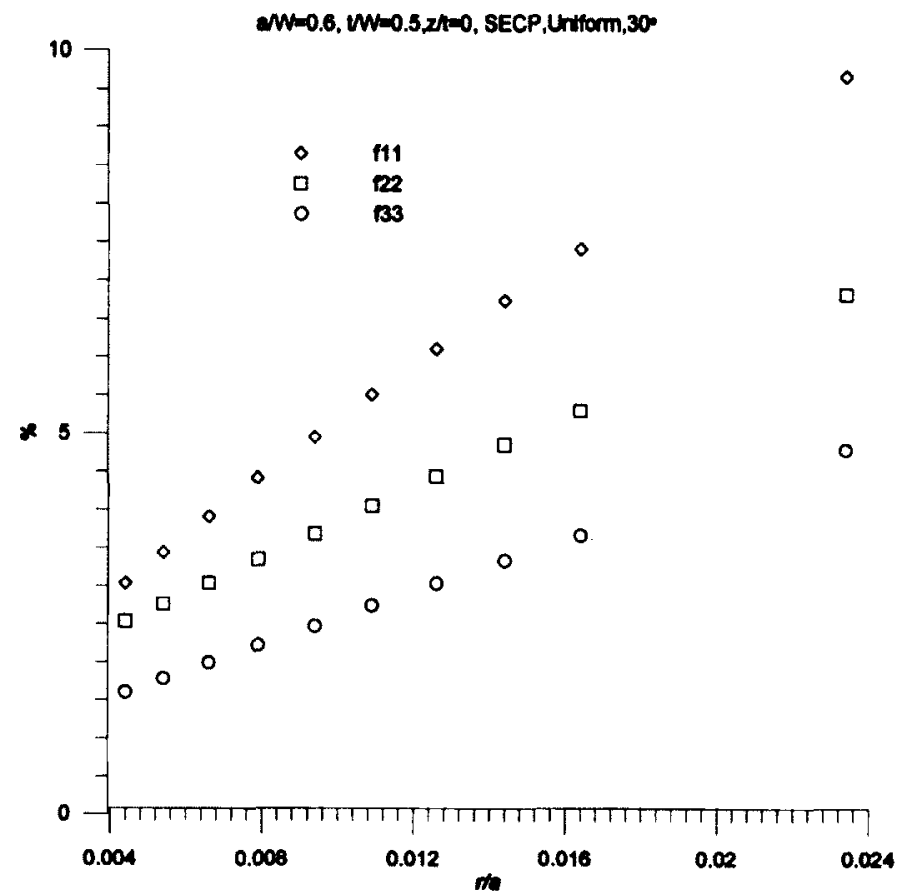

Figure 5.33 Percentage different varied by radius, $a / W=0.6, t / W=0.5$, Uniform, $30^{\circ}$ 


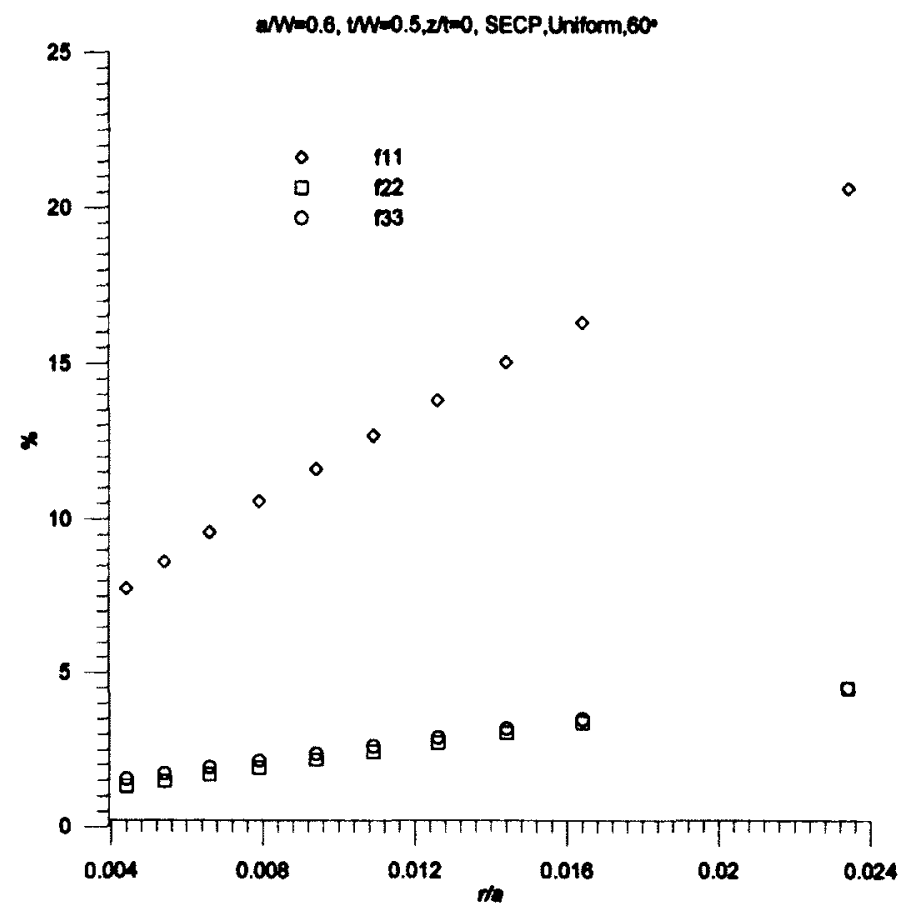

Figure 5.34 Percentage different varied by radius, $a / W=0.6, t / W=0.5$, Uniform, $60^{\circ}$

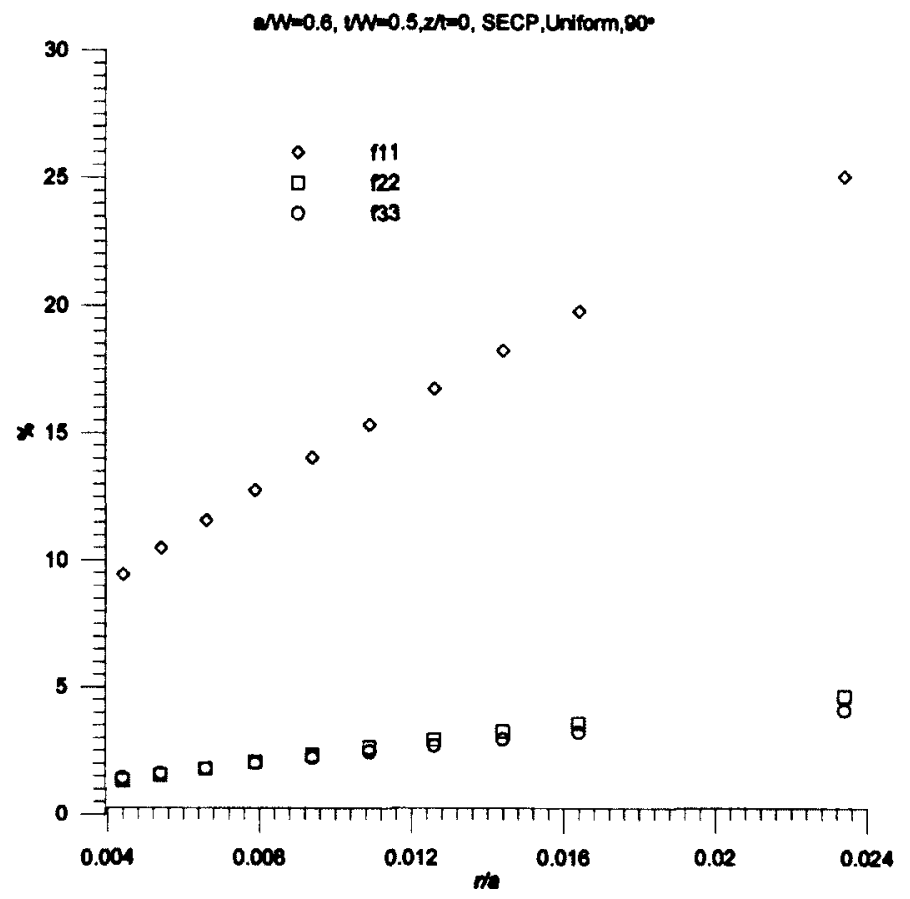

Figure 5.35 Percentage different varied by radius, $a / W=0.6, t / W=0.5$, Uniform, $90^{\circ}$ 


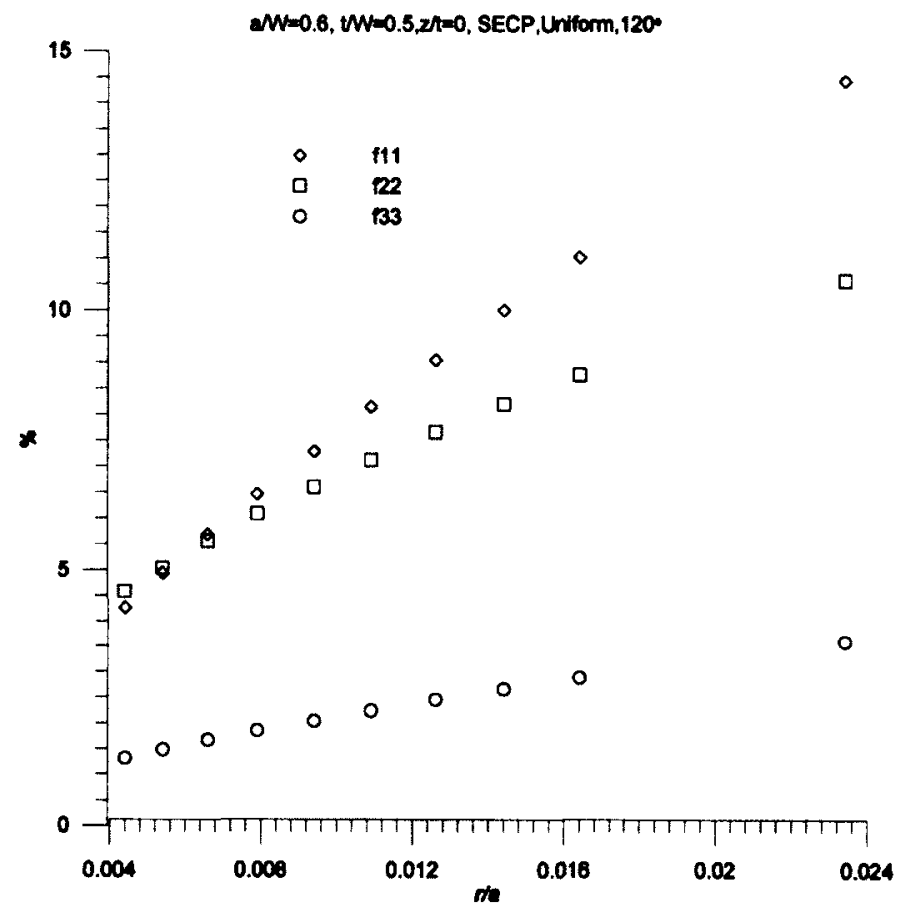

Figure 5.36 Percentage different varied by radius, $a / W=0.6, t / W=0.5$, Uniform, $120^{\circ}$

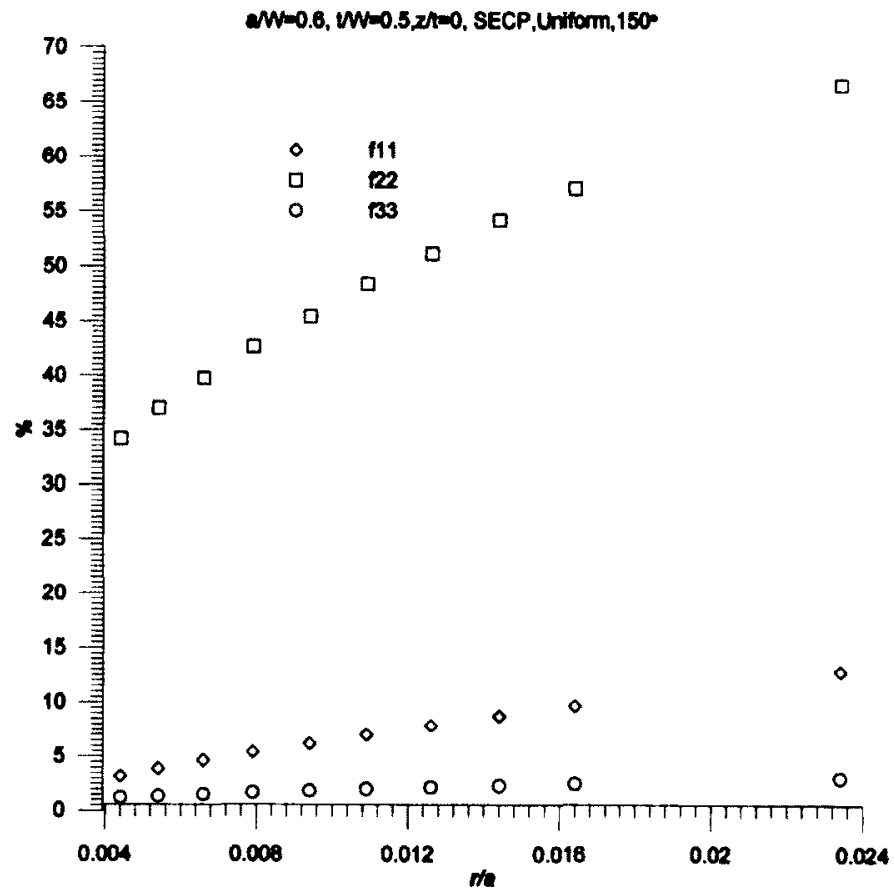

Figure 5.37 Percentage different varied by radius, $a / W=0.6, t / W=0.5$, Uniform, $150^{\circ}$ 


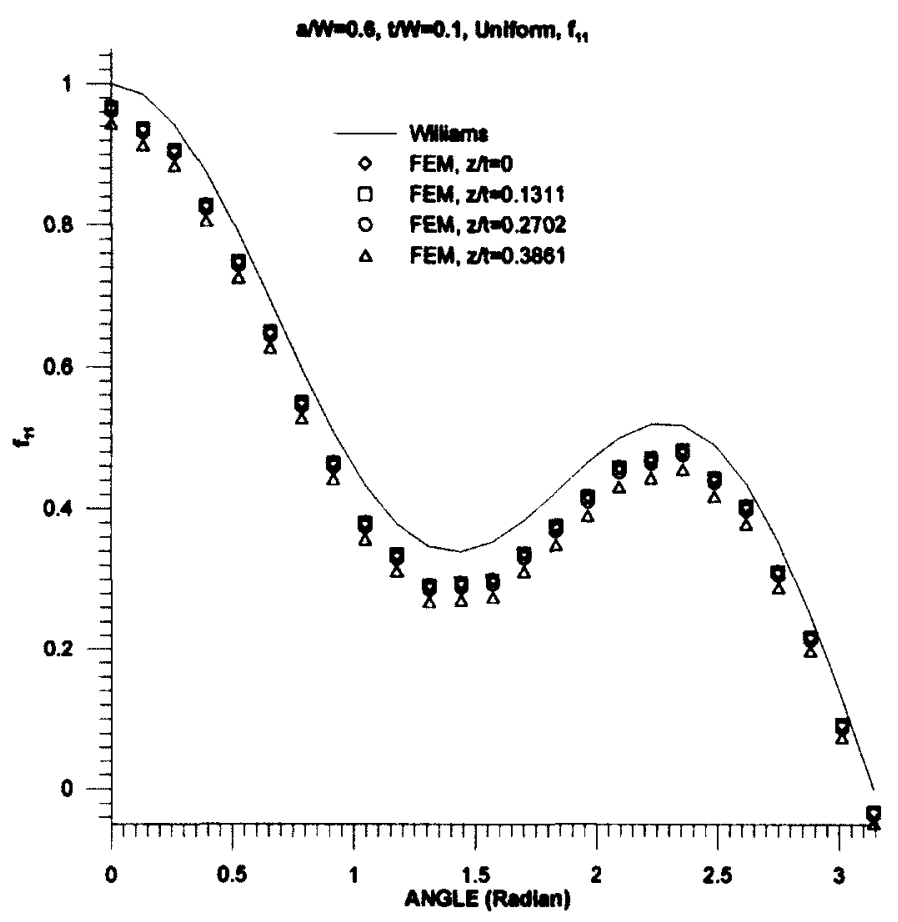

Figure 5.38 Stress State along the circular path close to the front varied by four locations along thickness, $a / W=0.6, t / W=0.1, r / a=0.0078, f_{11}$, Uniform, SECP

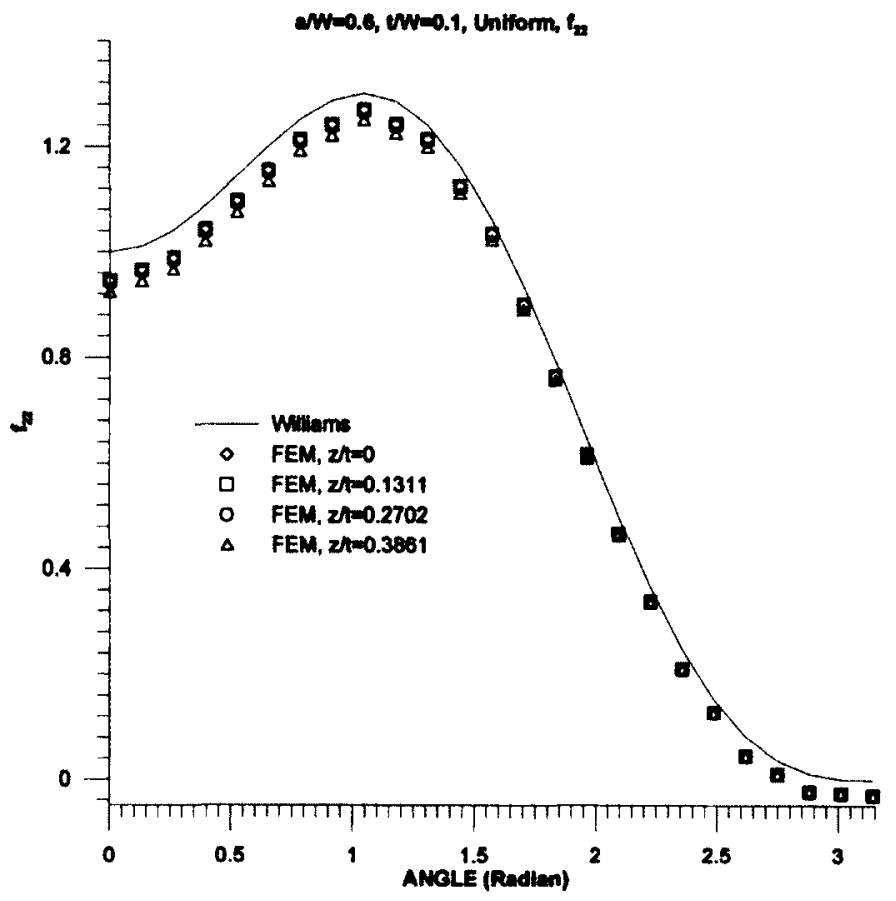

Figure 5.39 Stress State along the circular path close to the front varied by four locations along thickness, $a / W=0.6, t / W=0.1, r / a=0.0078, f_{22}$, Uniform, SECP 


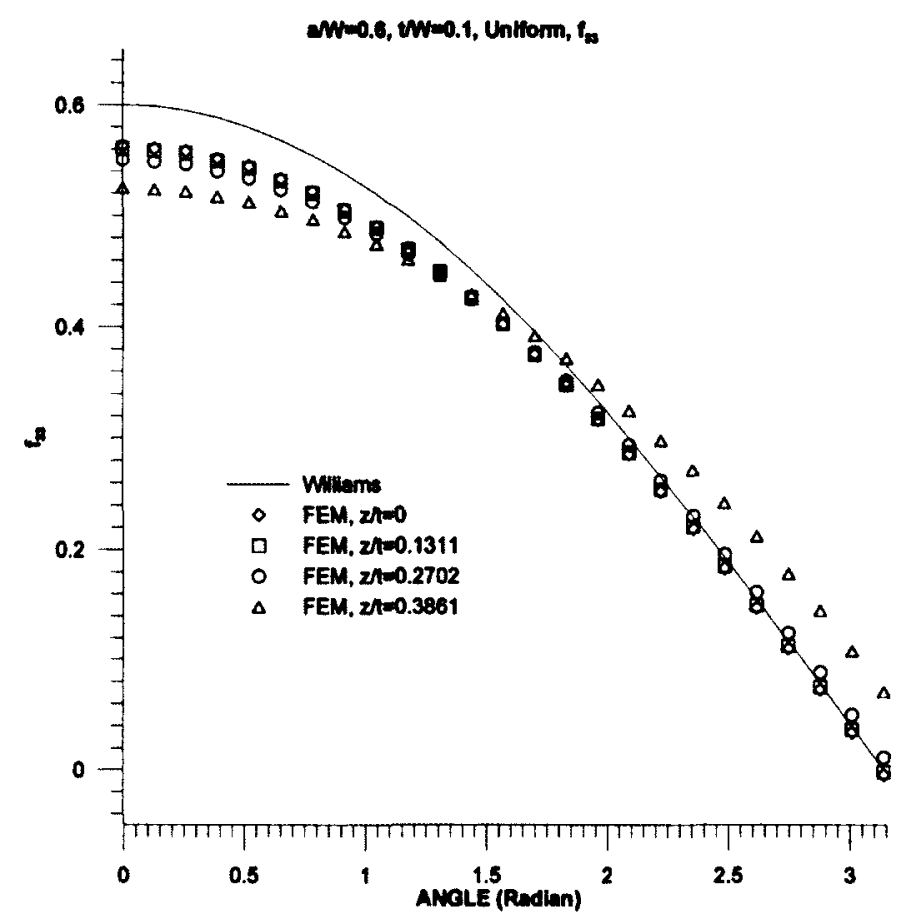

Figure 5.40 Stress State along the circular path close to the front varied by four locations along thickness, $a / W=0.6, t / W=0.1, r / a=0.0078, f_{33}$, Uniform, SECP

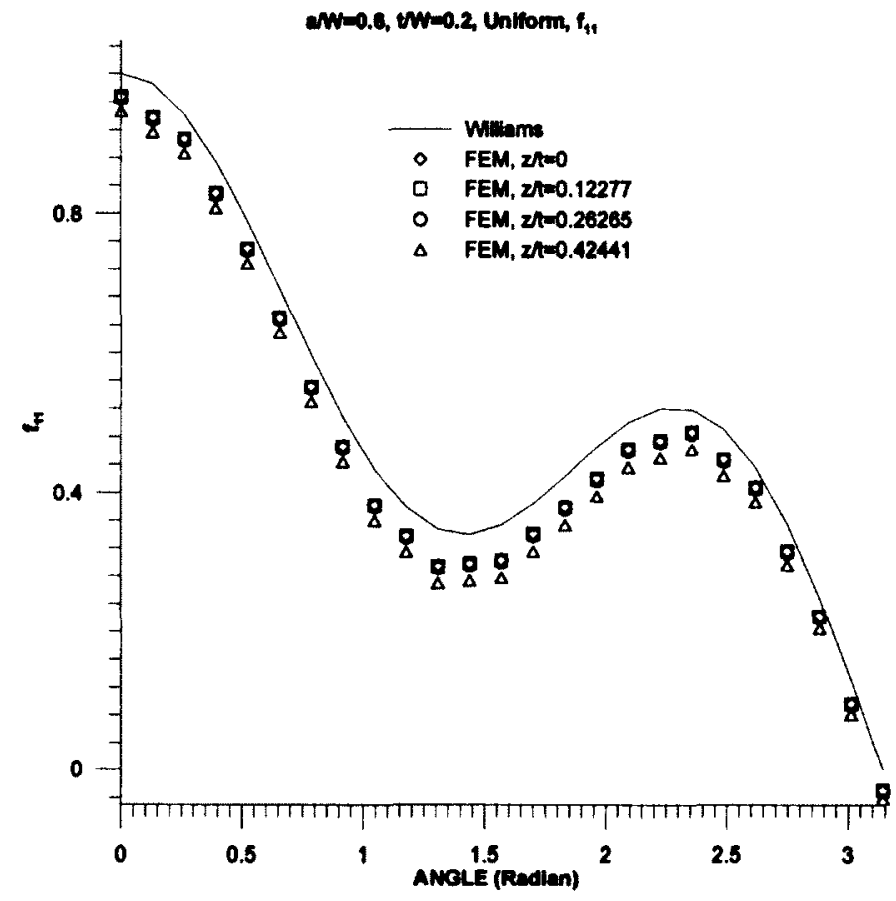

Figure 5.41 Stress State along the circular path close to the front varied by four locations along thickness, $a / W=0.6, t / W=0.2, r / a=0.0078, f_{l l}$, Uniform, SECP 


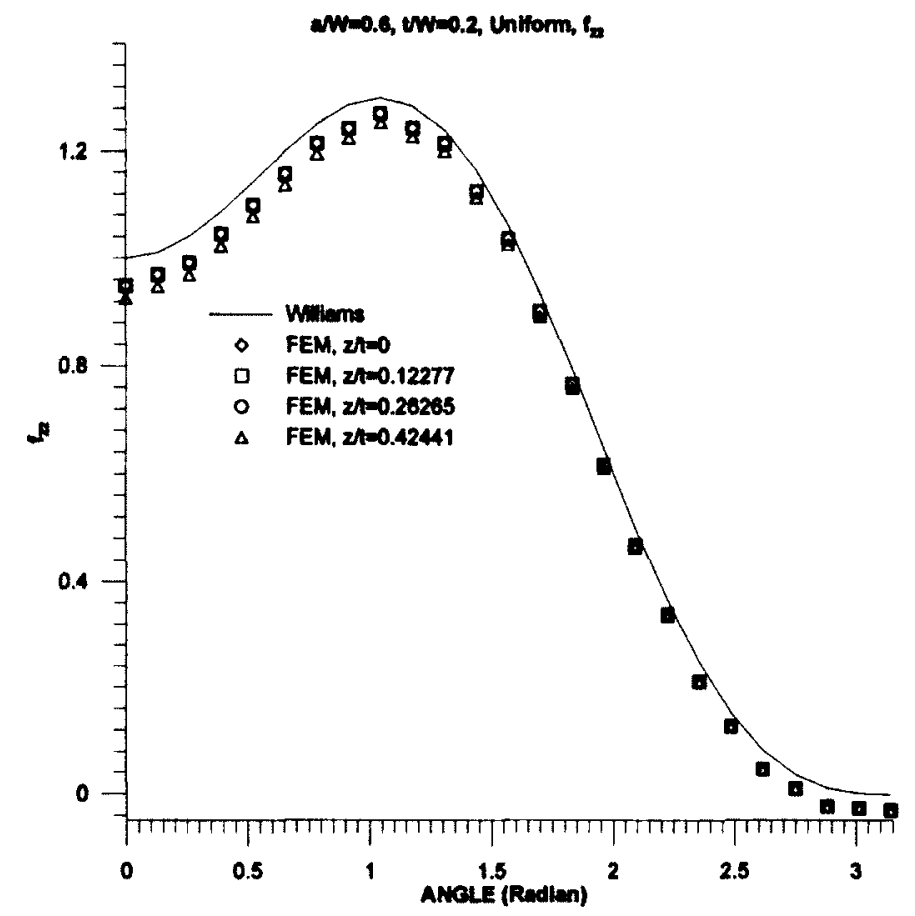

Figure 5.42 Stress State along the circular path close to the front varied by four locations along thickness, $a / W=0.6, t / W=0.2, r / a=0.0078, f_{22}$, Uniform, SECP

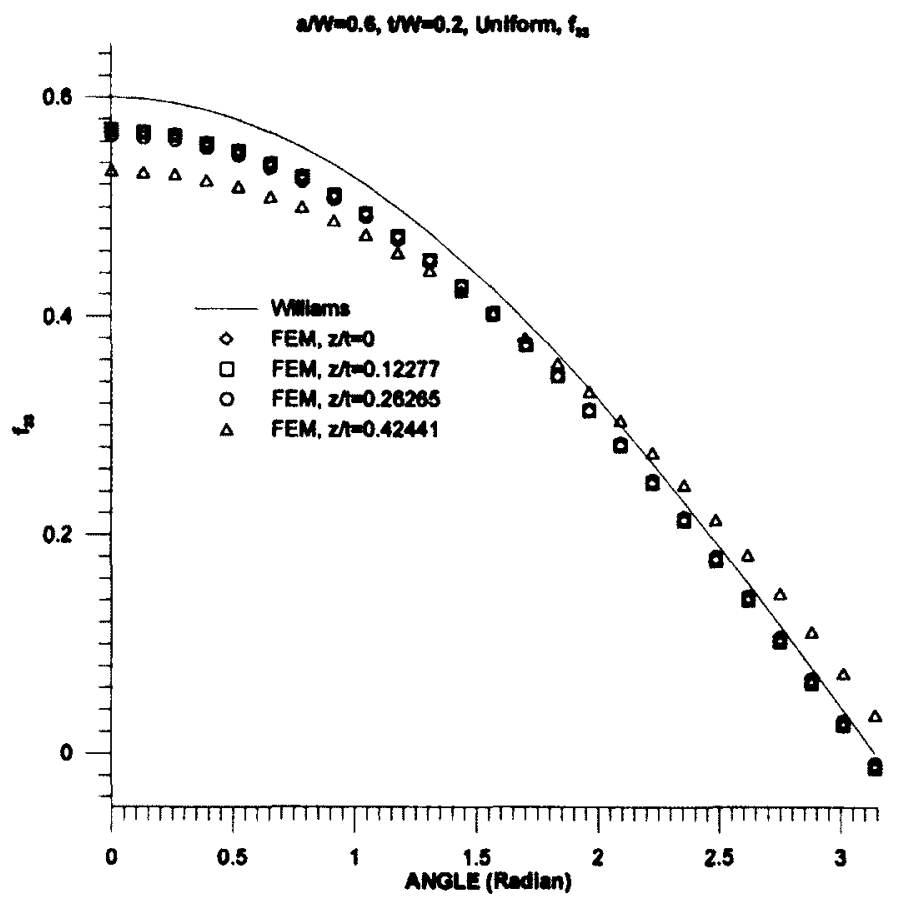

Figure 5.43 Stress State along the circular path close to the front varied by four locations along thickness, $a / W=0.6, t / W=0.2, r / a=0.0078, f_{33}$, Uniform, SECP 


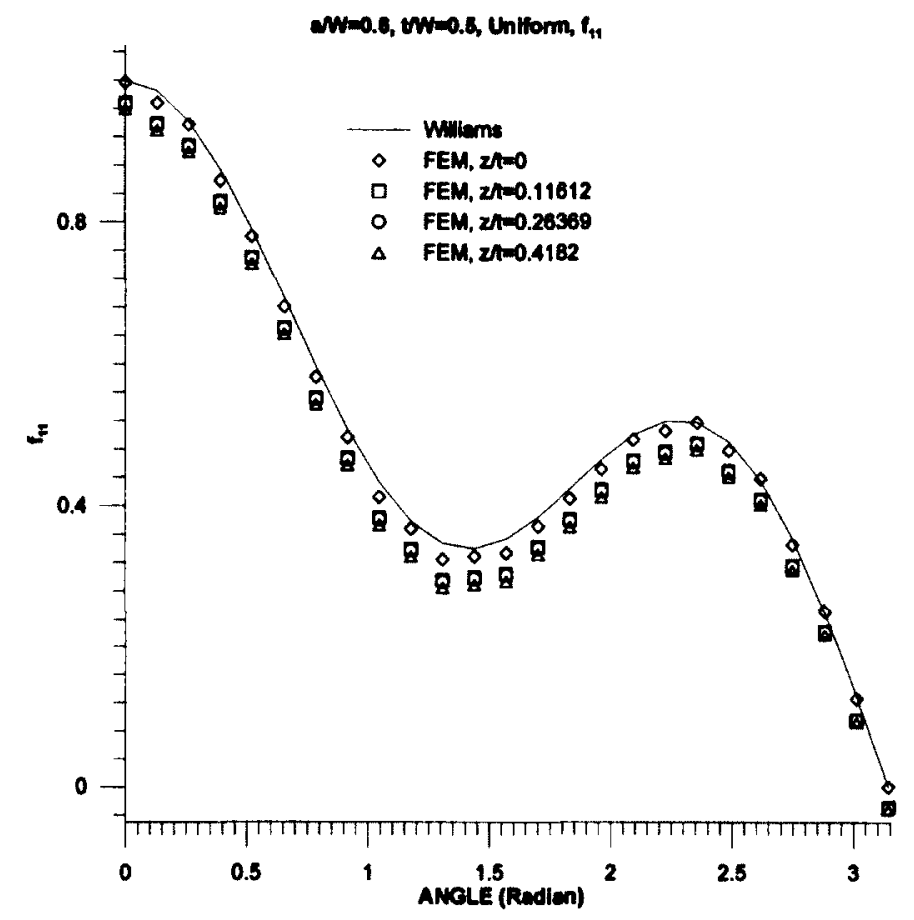

Figure 5.44 Stress State along the circular path close to the front varied by four locations along thickness, $a / W=0.6, t / W=0.5, r / a=0.0078, f_{11}$, Uniform, SECP

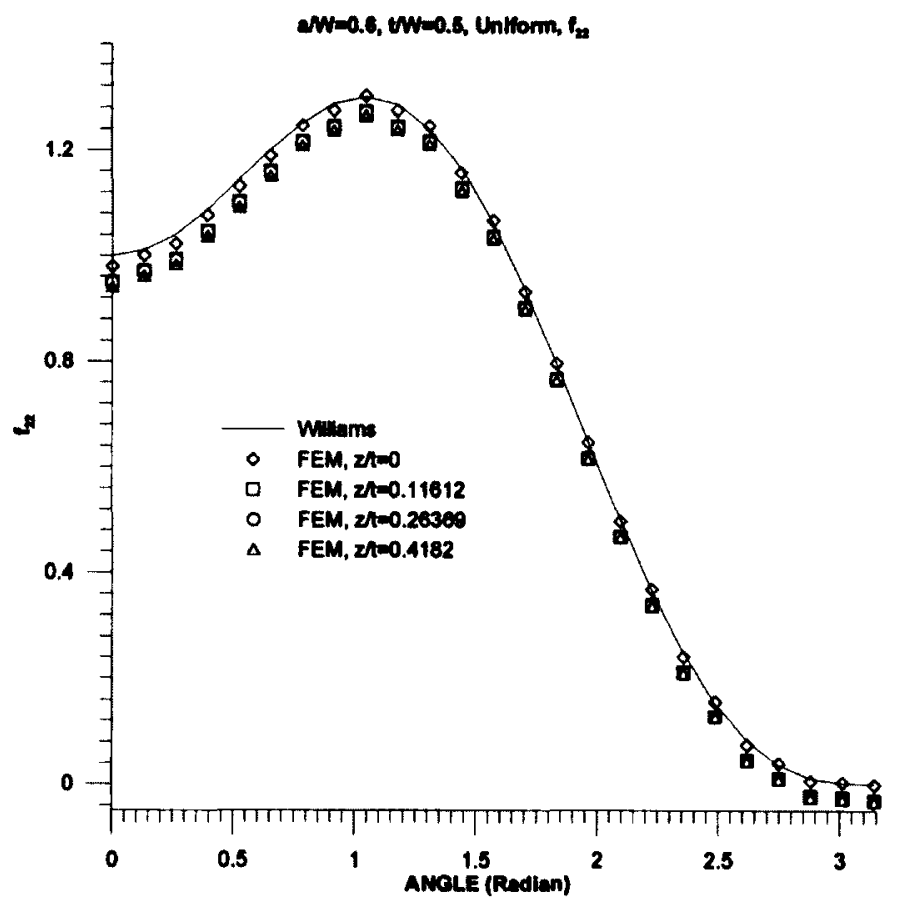

Figure 5.45 Stress State along the circular path close to the front varied by four locations along thickness, $a / W=0.6, t / W=0.5, r / a=0.0078, f_{22}$, Uniform, SECP 


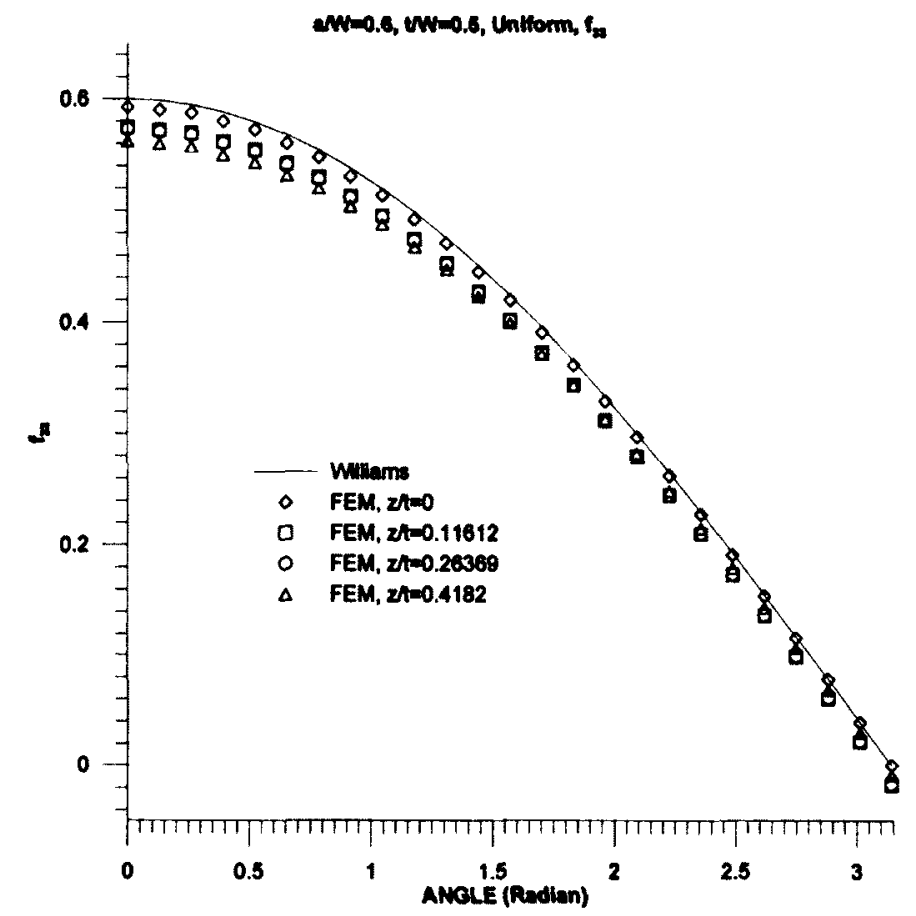

Figure 5.46 Stress State along the circular path close to the front varied by four locations along thickness, $a / W=0.6, t / W=0.5, r / a=0.0078, f_{33}$, Uniform, SECP

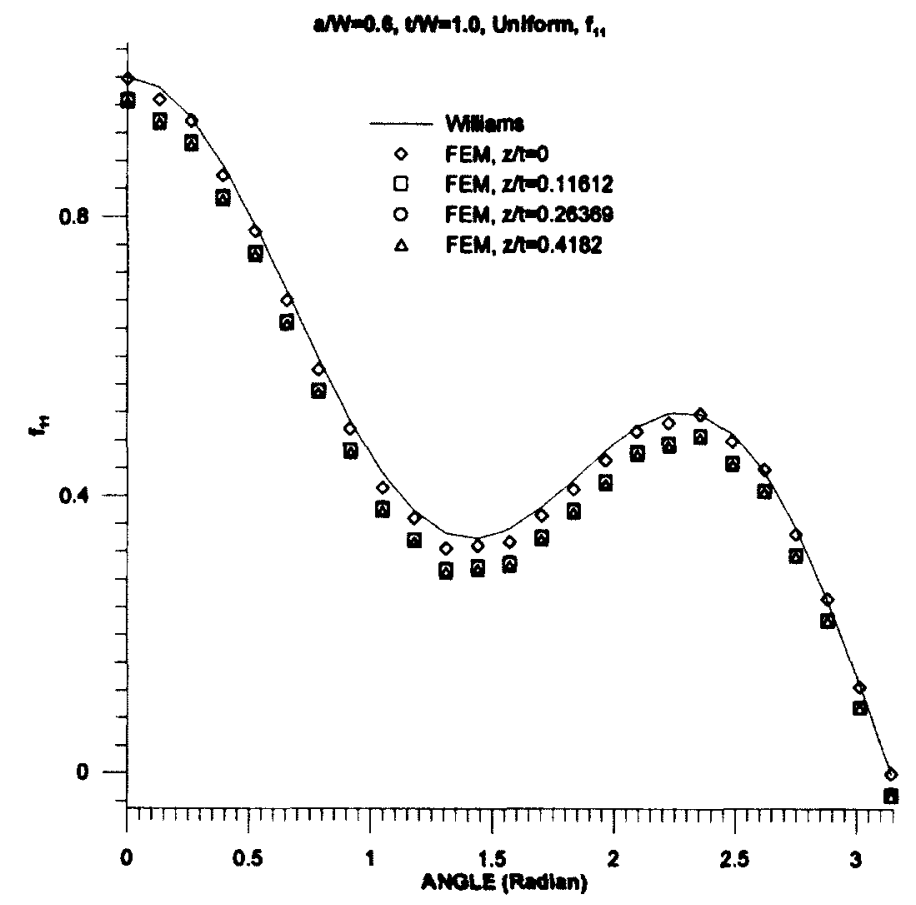

Figure 5.47 Stress State along the circular path close to the front varied by four locations along thickness, $a / W=0.6, t / W=1.0, r / a=0.0078, f_{11}$, Uniform, SECP 


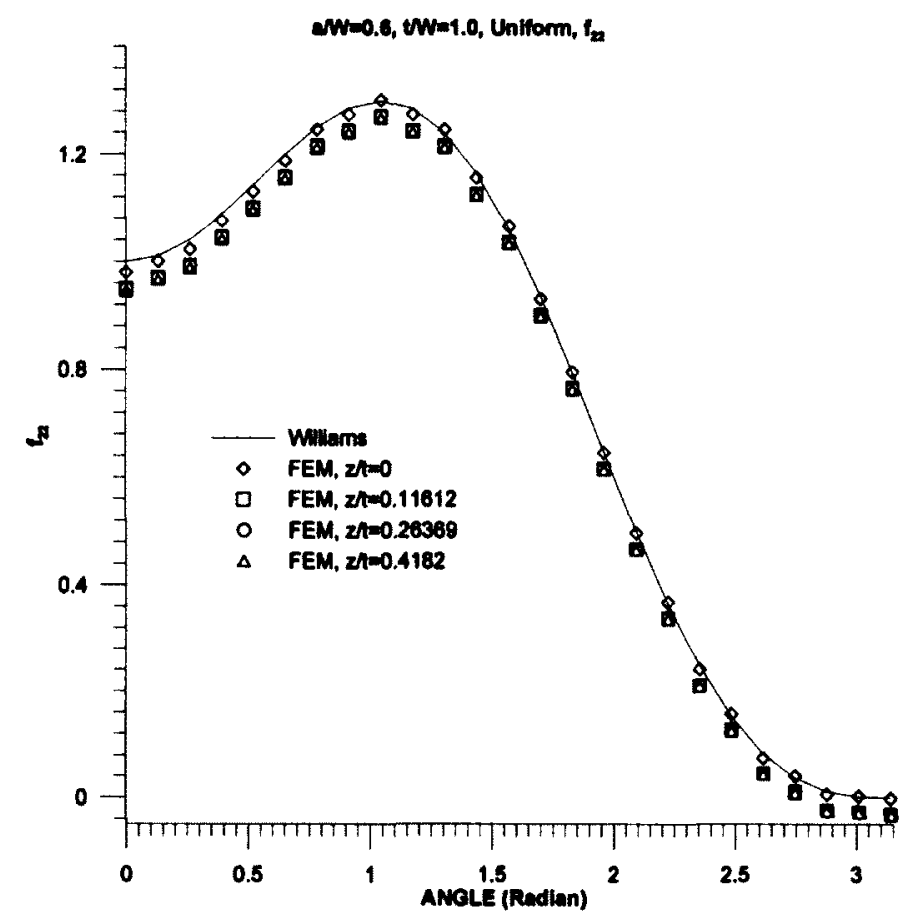

Figure 5.48 Stress State along the circular path close to the front varied by four locations along thickness, $a / W=0.6, t / W=1.0, r / a=0.0078, f_{22}$, Uniform, SECP

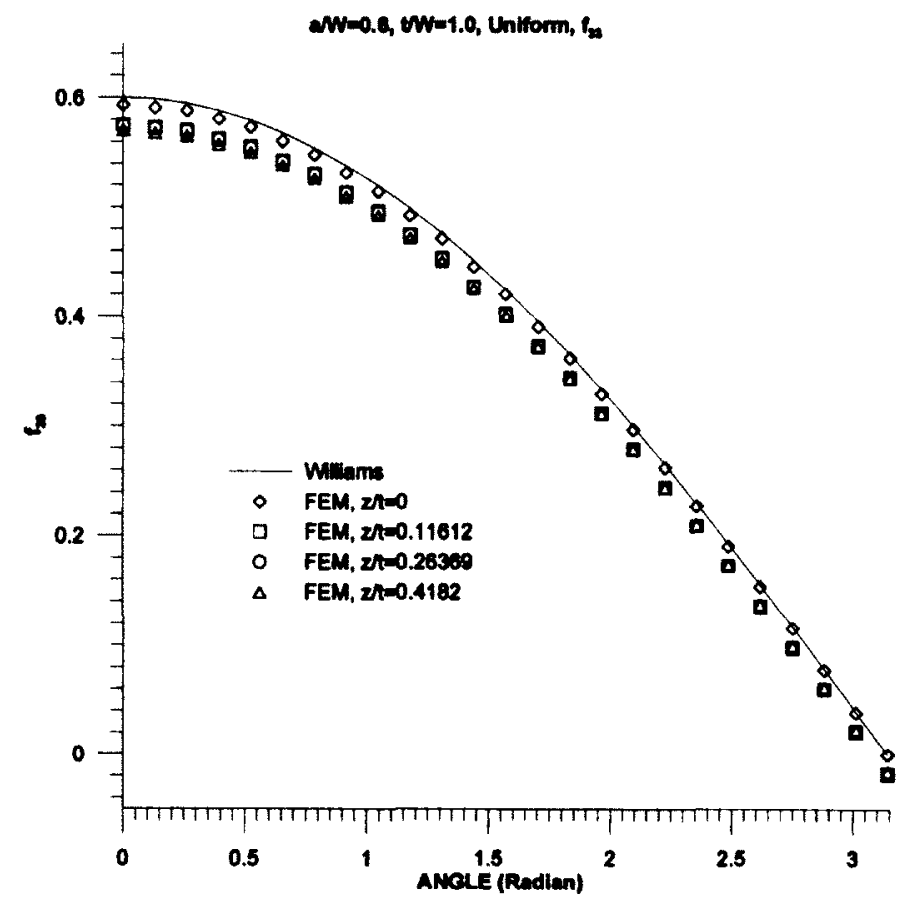

Figure 5.49 Stress State along the circular path close to the front varied by four locations along thickness, $a / W=0.6, t / W=1.0, r / a=0.0078, f_{33}$, Uniform, SECP 


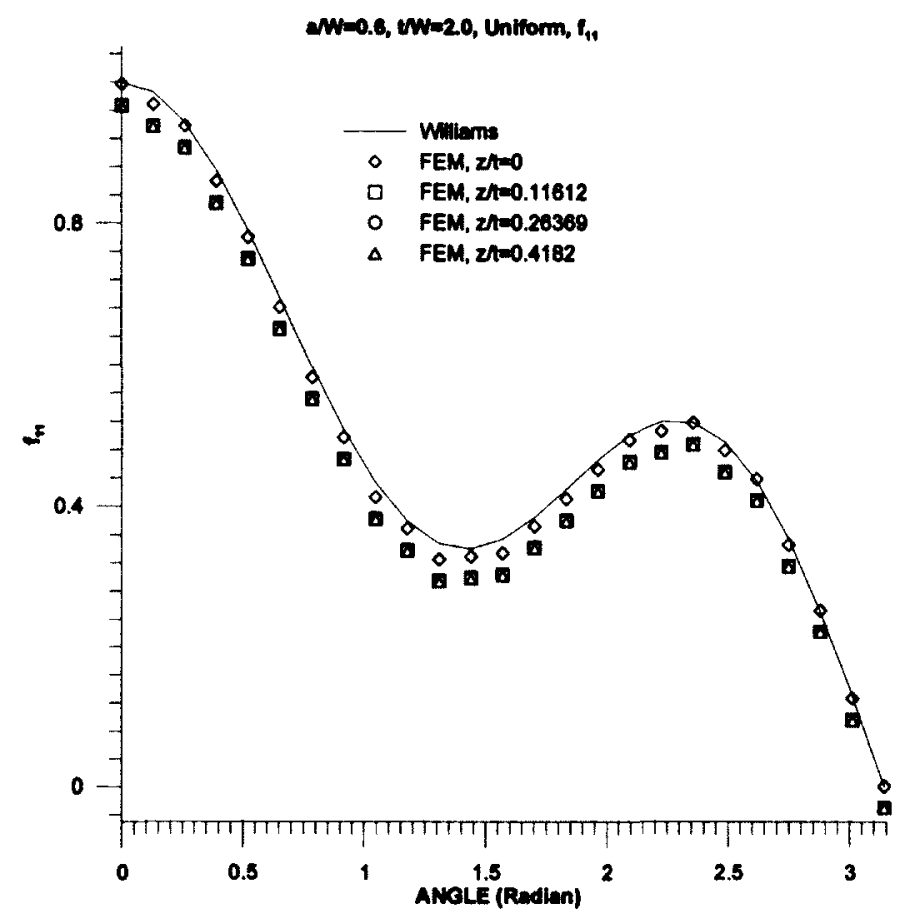

Figure 5.50 Stress State along the circular path close to the front varied by four locations along thickness, $a / W=0.6, t / W=2.0, r / a=0.0078, f_{11}$, Uniform, SECP

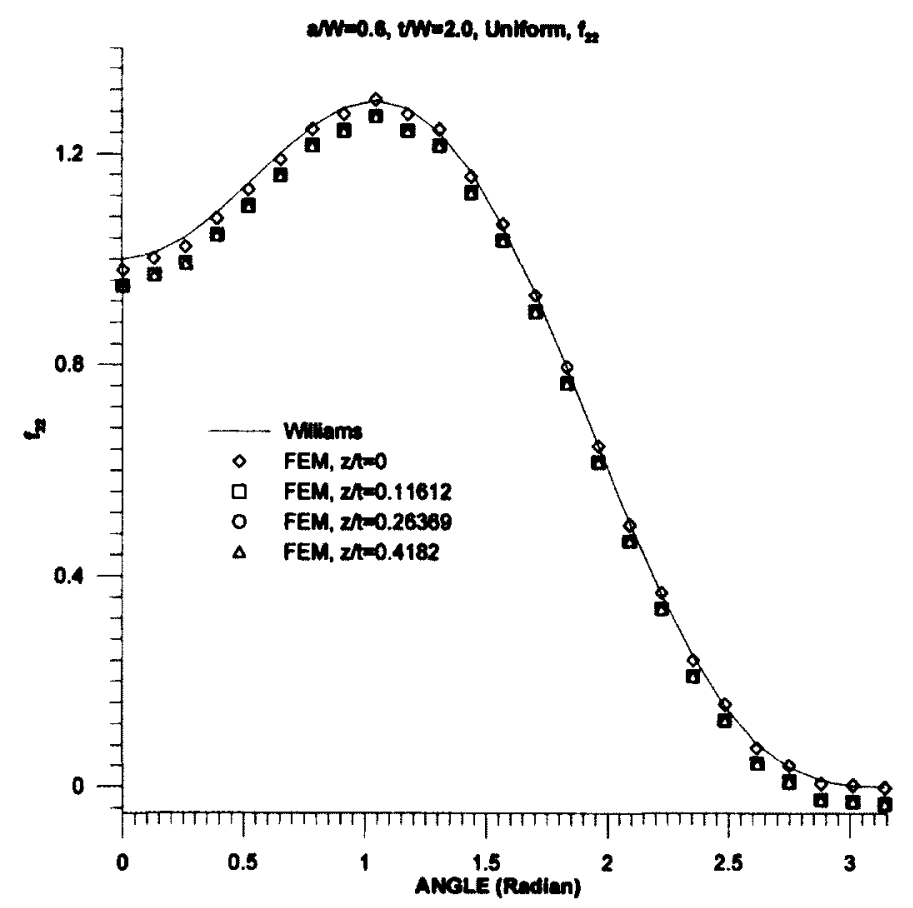

Figure 5.51 Stress State along the circular path close to the front varied by four locations along thickness, $a / W=0.6, t / W=2.0, r / a=0.0078, f_{22}$, Uniform, SECP 


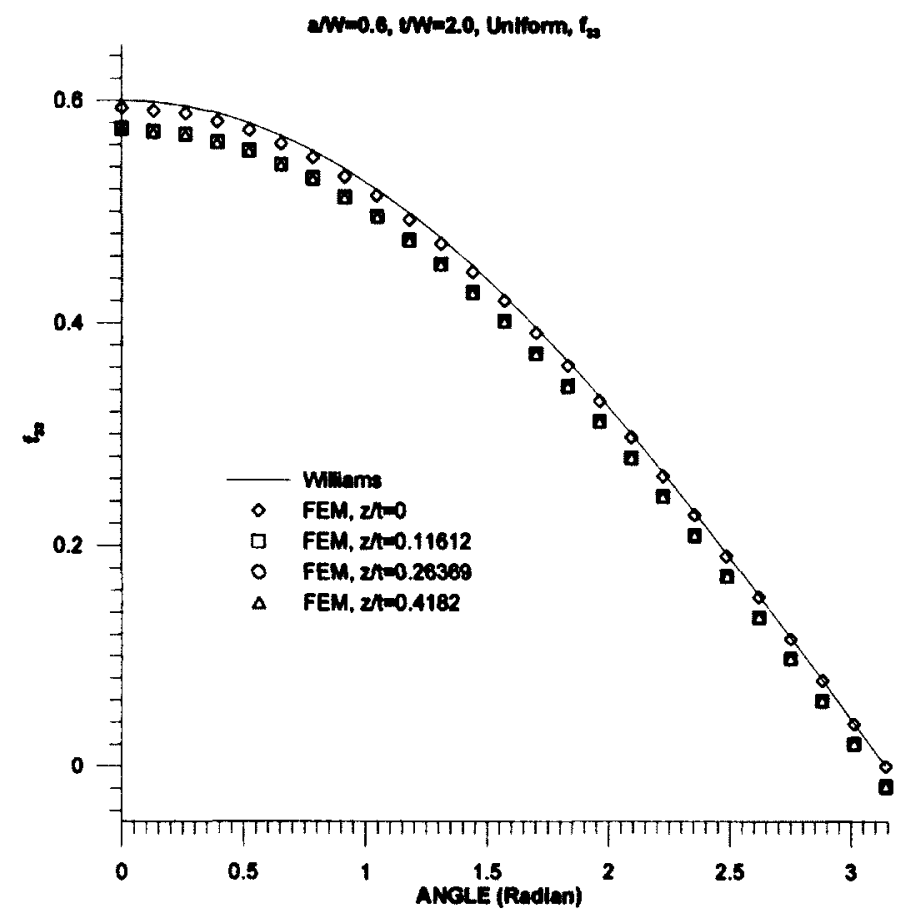

Figure 5.52 Stress State along the circular path close to the front varied by four locations along thickness, $a / W=0.6, t / W=2.0, r / a=0.0078, f_{33}$, Uniform, SECP

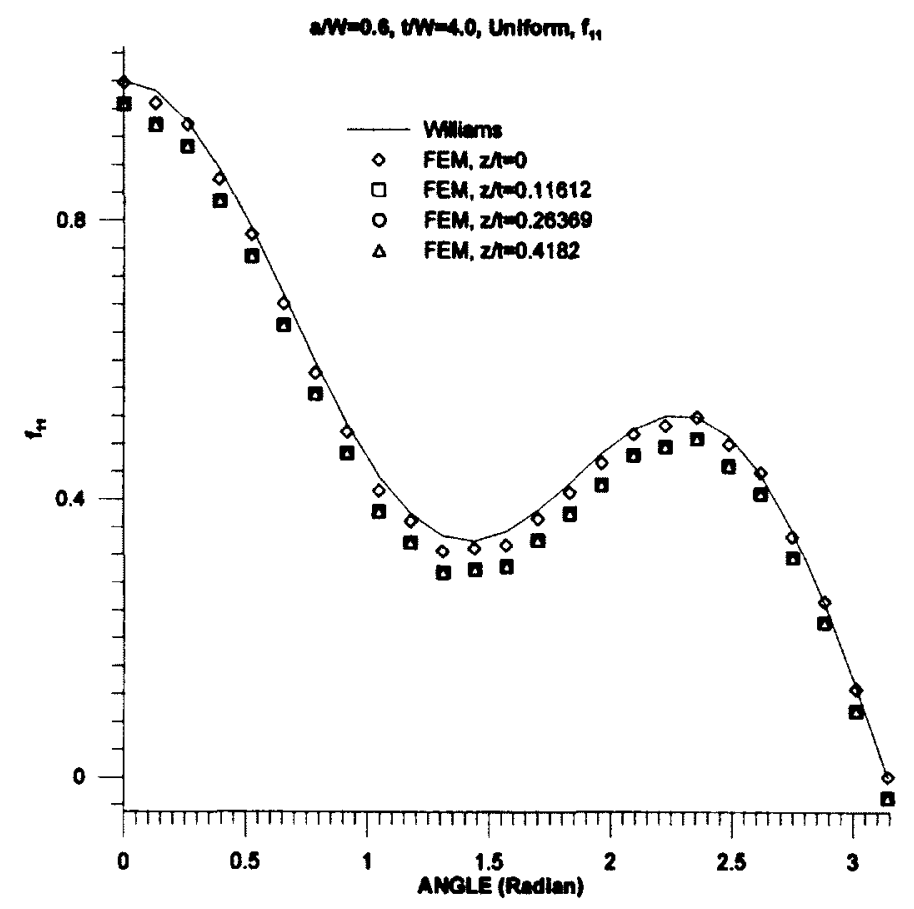

Figure 5.53 Stress State along the circular path close to the front varied by four locations along thickness, $a / W=0.6, t / W=4.0, r / a=0.0078, f_{l l}$, Uniform, SECP 


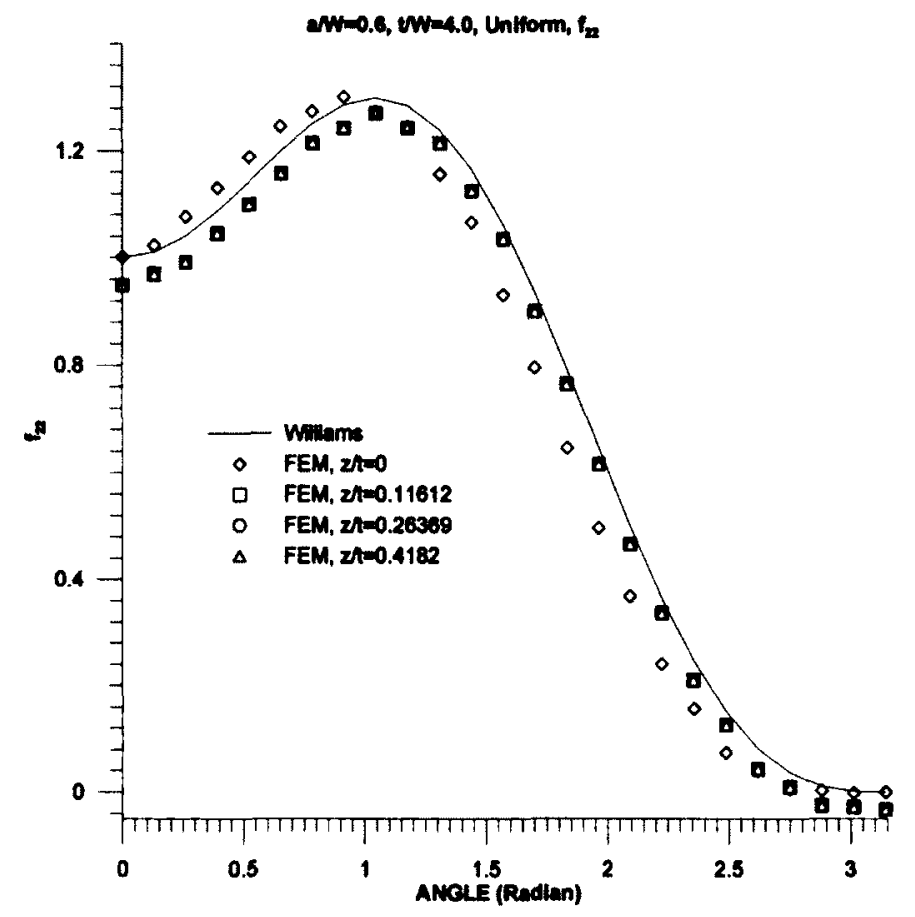

Figure 5.54 Stress State along the circular path close to the front varied by four locations along thickness, $a / W=0.6, t / W=4.0, r / a=0.0078, f_{22}$, Uniform, SECP

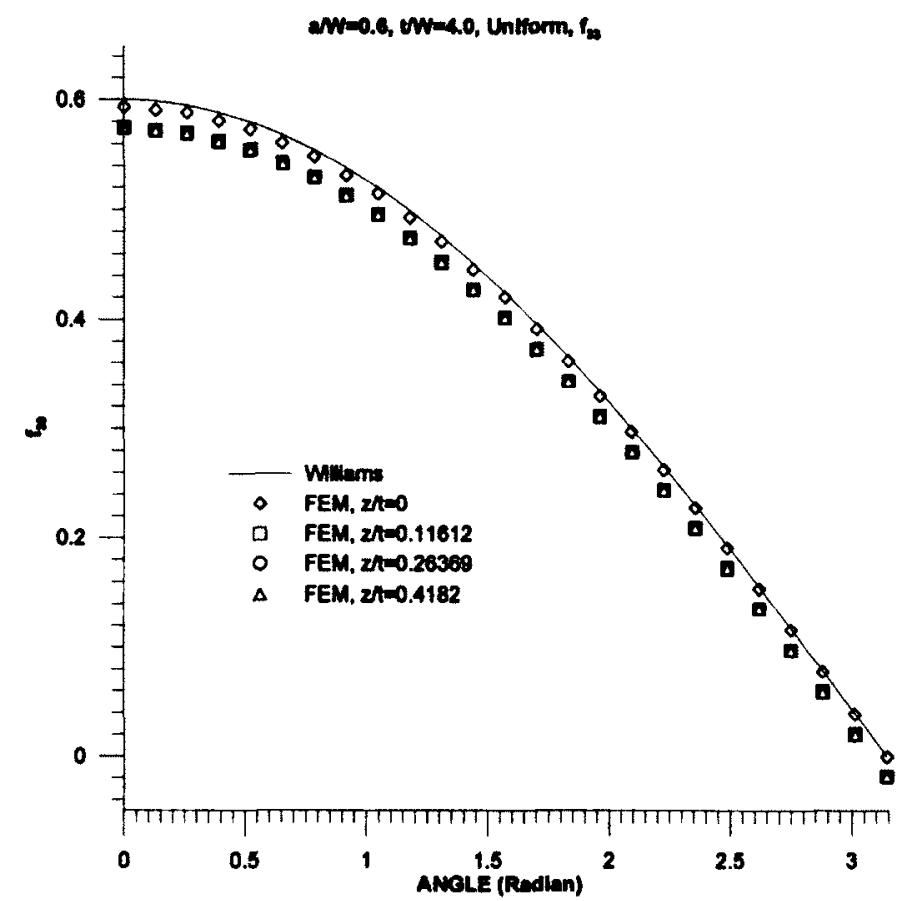

Figure 5.55 Stress State along the circular path close to the front varied by four locations along thickness, $a / W=0.6, t / W=4.0, r / a=0.0078, f_{33}$, Uniform, SECP 


\section{CHAPTER 6}

\section{CONCLUSIONS AND RECOMMENDATIONS}

\subsection{Conclusions}

The three-dimensional finite element analyses for a single edge cracked plate specimens with the crack surface subjected to uniform, linear, parabolic or cubic stress distribution were conducted. The stress intensity factor $K_{l}, T_{11}$ and $T_{33}$ stresses along the crack front were determined for cracked geometries with the relative crack depth $a / W$ of $0.2,0.4,0.6$ and 0.8 , and the relative thickness $t / W$ of $0.1,0.2,0.5,1.0,2.0$ and 4.0. For the convenience of engineering application, the empirical equations of the stress intensity factor $K_{l}, T_{11}$ and $T_{33}$ stresses at the mid-plane were given for uniform, linear, parabolic or cubic stress distribution applied on the crack surface.

The effect of the thickness on the stress intensity factor $K_{I}, T_{11}$ and $T_{33}$ stresses at mid-plane was discussed. Additional $t / W=0.0025,0.025,0.05,8,16$ cases subjected to the uniform stress were computed by finite element method in order to investigate the thickness effect. It is observed that the stress intensity factor $K_{I}$ at the mid-plane decreases when the plate is thicker, and trends to two-dimensional solution when the thickness becomes large compared to the crack length. It is noticed that the $T_{11}$ stress at mid-plane merge to two-dimensional solution when the thickness becomes large. A formula to predict the mid-plane $T_{11}$ stress developed by Nakamura and Parks (1992) was used to analyze by the finite element results. Very good accuracy was achieved between the finite element analyses and the predicted formula. It shows that the $T_{33}$ stress increases and approaches the two-dimensional solution as the plate thickness 
increases, and the three-dimensional results of $T_{33}$ stress gradually merge with the two-dimensional solution for thicker plate. Similar as the formula of $T_{11}$ stress, a formula to predict the mid-plane $T_{33}$ stress was proposed. The finite element analyses solution and the predicted formula of the mid-plane $T_{33}$ stress were compared. Reasonably agreements were achieved.

The effects of crack depth were studied for the stress intensity factor $K_{l}, T_{11}$ and $T_{33}$ stresses at mid-plane for various relative crack depths at $a / W=0.2,0.4,0.6$ and 0.8. It is noticed that the values of stress intensity factor all increase with increasing the crack depths whether the plane thickness is thick or thin. The $T_{11}$ stress is increasing at mid-plane for deeper crack at all kinds of the thickness cases. And the $T_{33}$ stress is increasing at mid-plane for deeper crack for larger thickness $(t / W>0.5)$ and decreasing for smaller thickness $(t / W=0.2)$.

The stress intensity factor $K_{I}, T_{11}$ and $T_{33}$ stresses varying along the crack front was investigated. For stress intensity factor $K_{l}$, the differences between the three-dimensional and two-dimensional solutions are within the range 0.95 to 1.07 for all cases of thickness, except in the small region near the free-surfaces. For $T_{11}$ stress, the three-dimensional and two-dimensional solutions are can be significantly different in the range of 1.5 to 1.8. For $T_{33}$ stress, three-dimensional solutions can be different than the two-dimensional in the factor of 10 to 50 . So the three-dimensional $T_{33}$ stresses solution must be applied to solve three-dimensional questions.

The stress state around the crack front in three dimensional SECP specimens is described by three parameters, stress intensity factor $K_{l}, T_{11}$ and $T_{33}$ stresses. The variation of the stress state of the Williams' expansion is compared with solutions of finite element analyses by angle, radius and thickness, respectively. And the percentages of difference between the finite element analyses and the Williams' expansion are also presented. It was observed the three parameters $K_{I}-T_{11}-T_{33}$ give good approximation of stress state close to the crack front (when $r / a$ is small). 


\subsection{Recommendations}

In the present thesis, the stress intensity factor $K_{l}, T_{11}$ and $T_{33}$ stresses of the single edge cracked plate specimens were calculated and investigated. However, there are also the other geometries of common specimens need to be studied, such as centre edge through-thickness cracked plate specimen and double edge through-thickness cracked plate specimen. Furthermore, the corner crack or semi-elliptical surface crack in finite thickness plate is also need to be systematicly studied.

The thinnest of the plate to calculate the stress intensity factor $K_{l}, T_{11}$ and $T_{33}$ stress at mid-plane is relative thickness $t / W=0.0025$, which the thinnest mesh by $3 \mathrm{D}$ solid element can be solved in ABAQUS for the models in this thesis. This leads to a limit to investigate the value of $B^{\text {thin }}$ in the prediction formula for $T_{11}$ stress or $B_{33}^{\text {thin }}$ in the prediction formula for $T_{33}$ stress. Only the prediction formula for $T_{11}$ and $T_{33}$ stresses at mid-plane is created, the other location of along the crack front should be analyzed in future. The prediction formula for $T_{33}$ stress is based on the prediction formula for $T_{11}$. However, the accuracy is not as good as the one for $T_{11}$. Further study is required on this topic. 


\section{REFERENCES}

ABAQUS Manual, 2008, Version 6.8. SIMULIA.

Barsoum, R. S. (1976). "On the use of isoparametric finite elements in linear fracture mechanics". International Journal for Numerical Methods in Engineering, Vol. 10, pp. 551-564.

Betegon, C. and Hancock, J. W. (1991). "Two-parameter characterization of elastic-plastic crack tip fields". ASME Journal of Applied Mechanics, Vol. 58, pp. 104-110.

Cardew, G. E. , Goldthorpe, M. R., Howard, I. C. and Kfouri, A. P. (1984). "On the elastic T-term". Fundamentals of Deformation and Fracture (Edited by Bilby, B. A., Miller, K. J. and Willis, J. R.), Cambridge University Press, Cambridge, pp. 465-476.

Chandler, D. (1989). "Metal fatigue seen as most likely cause". Boston Globe, 1.

Cotterell, B. and Rice, J.R. (1980). "Slightly curved or kinked cracks". International Journal of Fracture, Vol. 16, pp. 155-169.

Du, Z. Z. and Hancock, J. W. (1991). "The effect of non-singular stresses on crack tip constraint". Journal of Mechanical Physics and Solids, Vol. 39, pp. 555-567.

Fett, T. (1998). "Stress intensity factors and $T$-stress in edge-cracked rectangular plates under mixed boundary conditions". Engineering Fracture Mechanics, Vol. 60,pp. 625-630. 
Gao, H. (1992). "Variation of elastic T-stresses along slightly wavy 3D crack fronts". International Journal of Fracture, Vol. 58, pp. 241-257.

Goldthorpe, M. R. (1992). "Stress intensity factors and T-stresses for semi-elliptic cracks in plates subjected to bending". nCode International Report No. J2340/1 prepared for AEA Technology, November 1992.

Gonzalez-Albuixech, V. F., Ginear, E., Fernandez-Saez, J. and Fernandez-Canteli, A. (2011). "Influence of the $t_{33}$-stress on the 3-D stress state around corner cracks in an elastic plate". Engineering Fracture Mechanics, Vol. 78, pp. 412-427.

Griffith, A. A. (1921). "The phenomena of rupture and flow in solids". Philosophical Transactions of the Royal Society of London, A 221, pp. 163-198.

Gross, B. and Srawley, J. E. (1965). "Stress intensity factor for single-edge-notch specimens in bending or combined bending and tension". NASA TN D-2603.

Gross, B. and Srawley, J. E. (1965). "Stress intensity factor for three point bend specimens by boundary collocation". NASA TN D-3092.

Gross, B., Srawley, J. E. and Brown, W. F. (1965). "Stress intensity factor for a single-edge notch tension specimen by boundary collocation". NASA TN D-2396.

Henry, B.S. and Luxmoore, A.R. (1995). "Three-dimensional evaluation of the T-stress in centre cracked plates". International Journal of Fracture, Vol. 70, pp. 35-50.

Irwin, G. R. (1948). "Fracture dynamics". Fracturing of metals, pp. 147-166 ASM Publications. 
Irwin, G. R. (1958). "Fracture". Handbuch der Physik, Vol. VI, pp. 551-590. Berlin: Springer.

Kacianauskas, R., Zenon, M., Zarnovskij, V. and Stupak, E. (2005). "Three-dimensional correction of the stress intensity factor for plate with a notch". International Journal of Fracture, Vol. 136, pp. 75-98.

Kfouri, A. P. (1986). "Some evaluations of the elastic T-term using Eshelby's method". International Journal of Fracture, Vol. 30, pp. 301-315.

Kwon, S.W. and Sun, C.T. (2000). "Characteristics of three-dimensional stress fields in plates with a through-the-thickness crack". International Journal of Fracture, Vol. 104, pp. 291-315.

Larsson, S.G. and Carlsson, A.J. (1973). "Influence of non-singular stress terms and specimen geometry on small-scale yielding at crack tips in elastic-plastic material". Journal of the Mechanics and Physics of Solids, Vol. 21, pp. 263-278.

Leeves, P. S. and Radon, J. C. (1982). "Inherent stress biaxiality in various fracture specimen geometries". International Journal of Fracture, Vol. 9, pp. 311-325.

Meshii, T. and Tanaka, T. (2010). "Experimental $\mathrm{T}_{33}$-stress formulation of test specimen thickness effect on fracture toughness in the transition temperature region". Engineering Fracture Mechanics, Vol. 77, pp. 867-877.

Meshii, T., Tanaka, T. and Liu, K. (2010). "T-stress solutions for a semi-elliptical axial surface crack in a cylinder subjected to mode-I non-uniform stress distributions". 
Engineering Fracture Mechanics, Vol. 77, pp. 2467-2478.

Nakamura, T. and Parks, D.M. (1988). "Three-dimensional stress field near the crack front of a thin elastic plate". Journal of Applied Mechanics, Vol. 55, pp. 805-813.

Nakamura, T. and Parks, D.M. (1989). "Antisymmetrical 3-D stress field near the crack front of a thin elastic plate". International Journal of Solids and Structures, Vol. 25, No.12, pp. 1411-1426.

Nakamura, T. and Parks, D.M. (1992). "Determination of elastic T-stress along three-dimensional crack fronts using an interaction integral". International Journal of Solids and Structures, Vol.29, No.13, pp. 1597-1611.

O'Dowd, N. P. and Shih, C. F. (1991). "Family of crack tip fields characterized by triaxiality parameter- I". Structure of fields. Journal of Mechanical Physics and Solids, Vol. 39, pp. 989-1015.

Qu, J. and Wang, X. (2006). "Solutions of T-stresses for quarter-elliptical corner cracks in finite thickness plates subject to tension and bending". International Journal of Pressure Vessels and Piping, Vol. 83, pp. 593-606.

Raju, L.S. and Newman, J. C. (1977). "Three-dimensional finite-element analysis of finite-thickness fracture specimens". NASA TN D-8414.

Rice, J. R. (1968). “A path independent integral and the approximate analysis of strain concentration by notches and cracks". Journal of Applied Mechanics, Vol. 35, pp. 379-386. 
Rice, J.R. (1974). "Limitations to the small-scale yielding approximation for crack-tip plasticity". Journal of the Mechanics and Physics of Solids, Vol. 22, pp. 17-26.

Sham, T.-L. (1991). "The determination of the elastic T-term using higher order weight functions". International Journal of Fracture, Vol. 48, pp. 81-102.

Sherry, A.H., France, C.C. and Goldthorpe, M.R. (1995). "Compendium of T-stress solutions for two and three dimensional cracked geometries". Fatigue \& Fracture of Engineering Materials \& Structures, Vol. 18, No.1, pp. 141-155.

Sih, G.C. (1971). "A review of the three-dimensional stress problem for a cracked plate". International Journal of Fracture Mechanics, Vol. 7, pp. 39-61.

Timoshenko, S.P. and Goodier, J.N. (1970). "Theory of Elasticity". New York, McGraw-Hill.

Tracey, D.M. (1974). "Finite elements for three-dimensional elastic crack analysis". Nuclear Engineering and Design, Vol. 26, pp. 282-290.

Wang, X. (2002). "Elastic $T$-stress for cracks in test specimens subjected to non-uniform stress distributions". Engineering Fracture Mechanics, Vol. 69, pp. 1339-1352.

Wang, X. (2003). "Elastic T-stress solutions for semi-elliptical surface cracks in finite thickness plates". Engineering Fracture Mechanics, Vol. 70, pp. 731-756.

Wang, X. and Bell, R. (2004). "Ealstic T-stress solutions for semi-elliptical surface cracks in finite thickness plates subject to non-uniform stress distributions". Engineering 
Fracture Mechanics, Vol. 71, pp. 1477-1496.

Wang, Y.-Y. (1993). "On the two-parameter characterization of elastic-plastic crack front fields in surface cracked plates". In: Hackett E. M., Schwalbe K. H., Dodds R. H. editiors. Constraint Effects in Fracture, ASTM STP 1171. American Society for Testing and Materials; pp. 120-138.

Wang, Y.-Y. and Parks, D.M. (1992). "Evaluation of the elastic T-stresses in surface-cracked plates using the line-spring method". International Journal of Fracture, Vol. 56, pp. 25-40.

Williams, M. L. (1957). "On the stress distribution at the base of a stationary crack". Journal of Applied Mechanics, Vol. 24, pp. 111-114.

Yamamoto, Y. and Sumi, Y. (1974). "Stress intensity factors of twisted round bar with a circumferential crack". International Journal of Fracture, Vol. 10, pp. 269-271.

Yamamoto, Y. and Tokuda, N. (1973). "Determination of stress-intensity factors in cracked plates by the finite element method". International Journal for Numerical Methods in Engineering, Vol. 6, pp. 427-439.

Yamamoto, Y., Tokuda, N. and Sumi, Y. (1983). "Accuracy considerations for finite element calculations of the stress intensity factor by the method of superposition". Hybrid and Mixed Finite Element Methods, Eds. S. N. Atluri et al. (Wiley, New York, 1983), pp. 361-379. 
Yu, X. and Wang, X. (2005). "Weight functions for T-stresses for semi-elliptical surface cracks in finite-thickness plates". Journal of Strain Analysis, Vol. 40, pp. 403-419.

Zhao, J., Guo, W. and She, C. (2007). "Three-parameter description of the stress field near the border of an embedded elliptical crack". Acta Mechanica, Vol. 190, pp. 29-44. 